\title{
APPLICATION OF IMAGE FLOW CYTOMETRY AND PHOTOACOUSTICS FOR THE CHARACTERIZATION OF RED BLOOD CELL STORAGE LESIONS
}

by

Ruben N. Pinto

B.Sc. Hons., Ryerson University, 2014

\author{
A thesis \\ presented to Ryerson University \\ in partial fulfillment of the \\ requirements for the degree of \\ Master of Science \\ in the Program of \\ Biomedical Physics \\ Toronto, Ontario, Canada, 2017 \\ (C) Ruben N. Pinto 2017
}




\section{AUTHOR'S DECLARATION FOR ELECTRONIC SUBMISSION OF A THESIS}

I hereby declare that I am the sole author of this thesis. This is a true copy of the thesis, including any required final revisions, as accepted by my examiners.

I authorize Ryerson University to lend this thesis to other institutions or individuals for the purpose of scholarly research.

I further authorize Ryerson University to reproduce this thesis by photocopying or by other means, in total or in part, at the request of other institutions or individuals for the purpose of scholarly research.

I understand that my thesis may be made electronically available to the public.

Ruben N. Pinto 
Abstract

\title{
Application of image flow cytometry and photoacoustics for the characterization of red blood cell storage lesions
}

\author{
Ruben N. Pinto \\ M. Sc. Biomedical Physics \\ Ryerson University, Toronto, 2017
}

Significant functional/structural changes of red blood cells (RBCs) have been documented during its in vitro storage. Collectively termed as RBC storage lesions, changes include an increase in $\mathrm{RBC}$ oxygen saturation $\left(\mathrm{SO}_{2}\right)$ and an increase in irreversibly damaged $\mathrm{RBCs}$ (spheroechinocytes). In this work, novel optical techniques are presented for determining the spheroechinocyte population as a function of storage time via automated image flow cytometry (IFC) morphology characterization, and the acquisition of $\mathrm{RBC} \mathrm{SO}_{2}$ via an in situ photoacoustic (PA) method. Blood gas analysis (BGA) was used as the gold standard $\mathrm{SO}_{2}$ measure. Over the lifespan of seven blood bags, the IFC spheroechinocyte population - $\mathrm{PA} \mathrm{SO}_{2}$ correlation was found to be strong $\left(0.60<\mathrm{r}^{2}<0.91\right)$. These results suggest that monitoring $\mathrm{SO}_{2}$ changes can potentially infer the rate of increase of irreversibly damaged RBCs. A very strong $\mathrm{PA} \mathrm{SO}_{2}-$ BGA $\mathrm{SO}_{2}$ correlation $\left(\mathrm{r}^{2}>0.95\right)$ shows high potential for in situ monitoring of $\mathrm{RBC}$ storage lesions. 


\section{Acknowledgements}

There are several individuals that I would like to acknowledge for contributing to various aspects of this project. Firstly, I would like to thank members of the Kolios and BioClinical Optics \& Photonics (BCOP) groups for the scientific interactions and discussions that helped guide my work. Specifically, I would like to acknowledge the following people for their help throughout various stages of the project: Homa Assadi, for her guidance in working with spectrophotometers; Kirsten Cardinell, with whom the first prototype setup was developed for photoacoustic measurements of blood bags in situ; Muhannad Fadhel, whose advice, discussions and help in developing a protocol for photoacoustic oxygen saturation acquisition have been very valuable to the project; Karan Bagga, for his assistance with the design of the photoacoustic blood bag container and the development of experimental protocols implemented for the blood gas analyzer and photoacoustic measurements; Joseph Sebastian, for his help with the development of the image segmentation template on IDEAS ${ }^{\circledR}$, as well as analysis pertaining to image flow cytometry data; and Elizabeth Berndl, who helped establish blood bag and handling protocols. I am grateful for the day-to-day support provided by all these wonderful people.

I would like to acknowledge the following individuals, whose technical support was critical for the development of major aspects of the project: Arthur Worthington, who helped build the photoacoustic blood bag container; Danielle Gifford, for her help in establishing a syringe-based blood gas analyzer assay; Angela Hill, for the training provided in blood bag handling, extraction and red blood cell assays; Tracey Turner, for providing her expertise in morphologically characterizing red blood cell images acquired from the image flow cytometer; Michael Parsons, whose insight and proficiency was crucial in the development of the image flow cytometry assay; and Tim Chang, whose work on the image segmentation template provided a level of robustness that allowed for an accurate determination of spheroechinocyte populations. I sincerely thank all of them for donating their time and high-level expertise to enhance this project.

I was very fortunate to interact with the following people whose knowledge, experience and scientific intuition were extremely valuable, especially when tackling many issues impeding the progress of the project. I would like to acknowledge Eno Hysi, Dr. William Whelan, Dr. General Leung and Dr. Jason Acker for graciously offering their time and resources to help me fill gaps 
in knowledge and overcome several roadblocks. Along with Dr. Whelan, I would also like to thank Dr. Carl Kumaradas for providing me scientific and professional feedback as committee members. Their scholarly criticism and suggestions helped me grow as a researcher throughout the project.

I would like to thank my co-supervisor Dr. Alexandre Douplik, who was always ready to provide his knowledge and experience for the development of various optical techniques associated with the project. He allowed me to develop more than just my research skills, by providing opportunities to manage the BCOP group in several ways. I sincerely thank him for the resources provided throughout this project.

I would like to acknowledge my primary supervisor, Dr. Michael Kolios, for providing me with such an incredible research opportunity. His passion, guidance and scientific intuition inspired me and motivated me to achieve many goals within the project. I sincerely thank him for the resources he allocated to allow me to pursue unexplored territories, of which some pathways lead to important findings that are central to this thesis. I also thank him for his tireless efforts to provide quality feedback on all aspects of this project; his approach to scientific research and professionalism has helped me grow tremendously as a scientist. It was an honor working in his lab and under his supervision.

Lastly, I would like to acknowledge my family members, who have provided me with encouragement and love that is impossible to quantify. To my mom, dad, brothers and wife, I cannot thank you enough for all the guidance and support you have given me through all these years, while I chased after my academic goals.

To my loving family members, dear friends and supportive colleagues, a sincere and hearty thank you to all mentioned above. 
To my wife, Dolly:

I have accomplished what I have because of you, Your love got me through the stormy weather. I'm looking forward to your book review, and writing the next chapter together. 


\section{Contents}

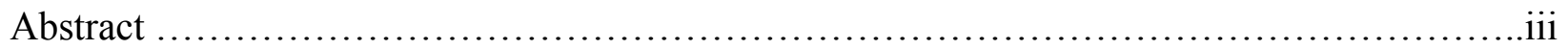

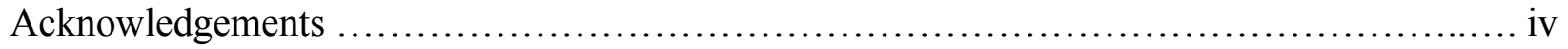

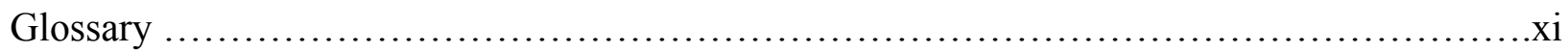

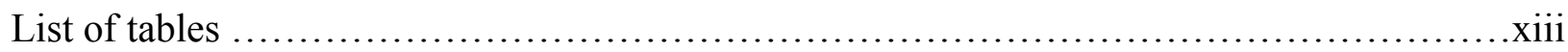

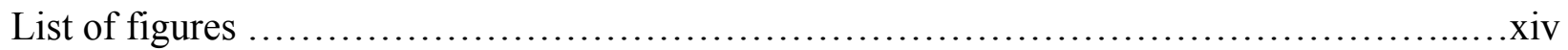

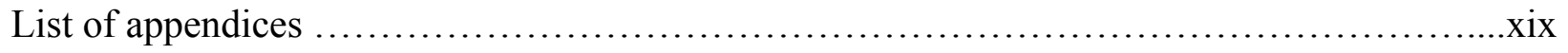

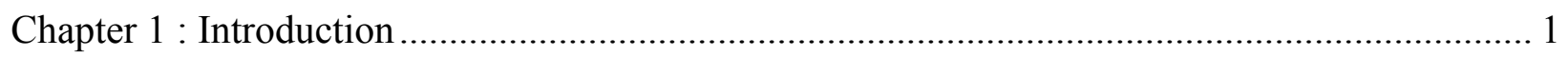

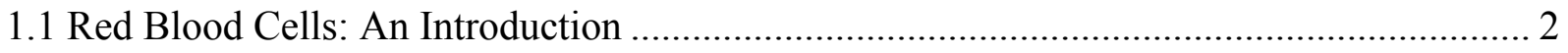

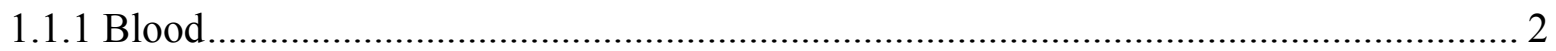

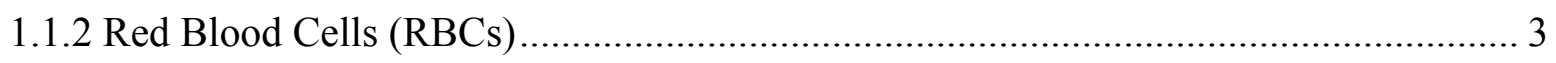

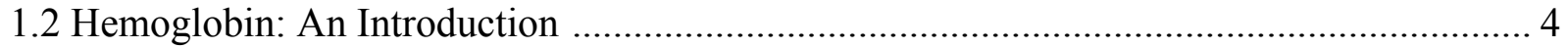

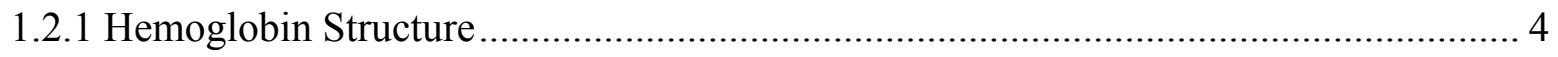

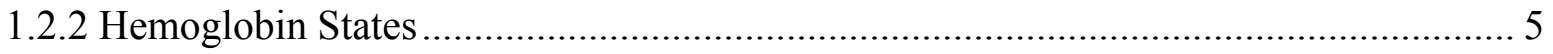

1.2.3 Oxygen Saturation, Oxygen Dissociation Curve and Cooperative Binding .................. 6

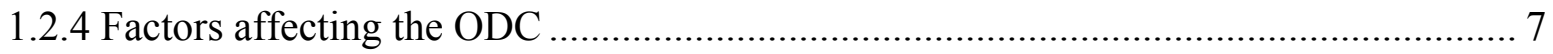

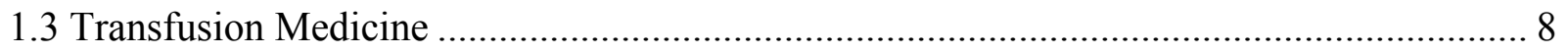

1.3.1 Canadian Transfusion Management .......................................................................... 9

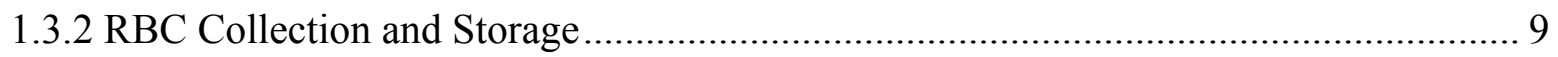

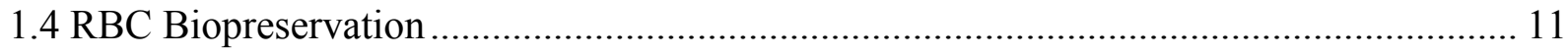

1.4.1 In vitro Hemolysis and In vivo Survivability ............................................................ 11

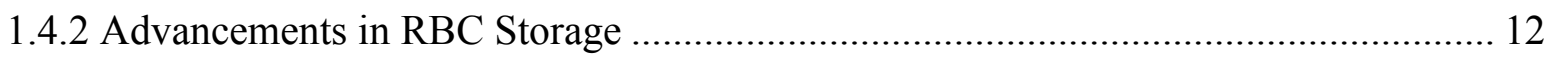

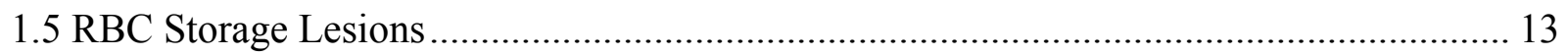

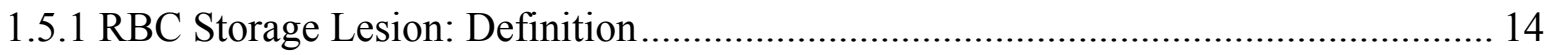

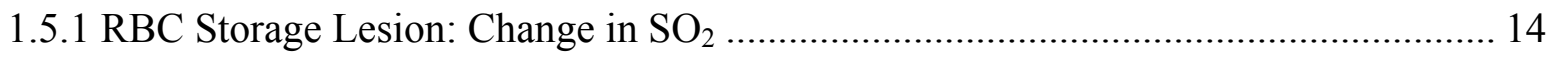

1.5.3 RBC Storage Lesion: Change in Morphology …………………............................ 17

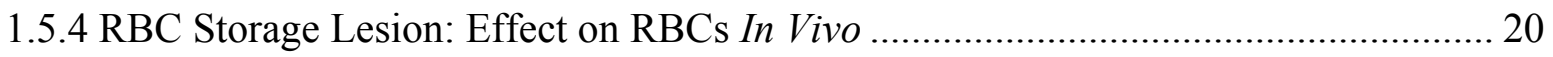

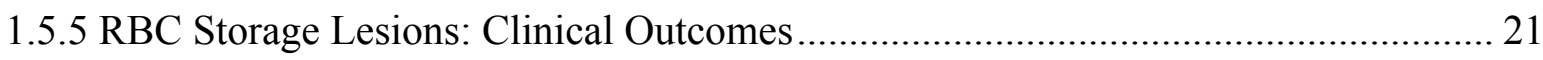

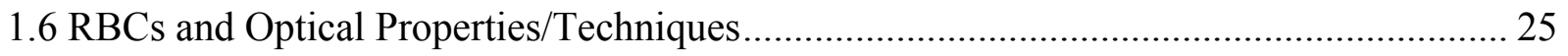

1.6.1 Optical Parameters of RBCs at the VIS-NIR Optical Regime ……………............... 25

1.6.2 Absorption Spectroscopy and Hemoglobin Concentration ........................................... 26 


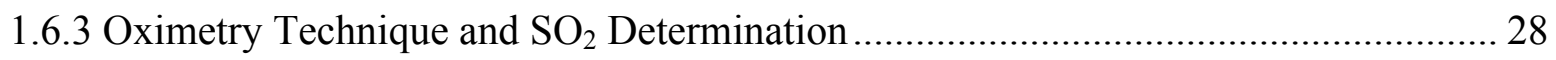

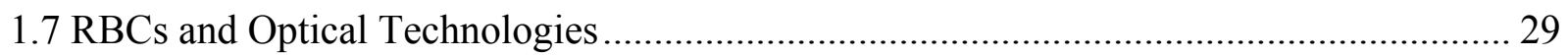

1.7.1 Transmitted Light Microscopy and RBC Morphology Assay ..................................... 29

1.7.2 Spectrophotometry and Hemoglobin-Related Assays ................................................. 30

1.7.3 Flow Cytometry and RBC Fluorescent Staining Assays ............................................ 32

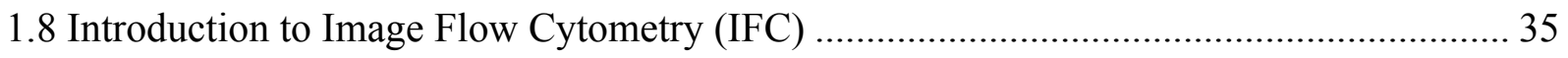

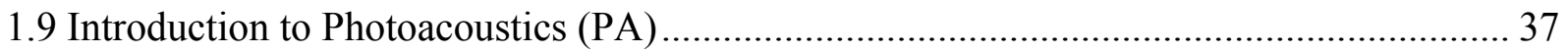

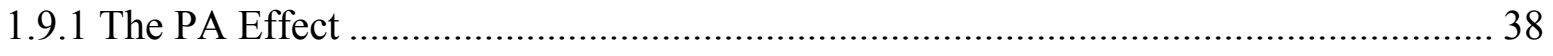

1.9.2 Advantages over Conventional Optical/Ultrasound Technologies............................... 40

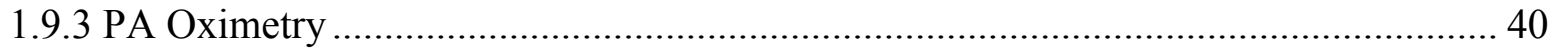

1.9.4 Potential for non-invasive $\mathrm{SO}_{2}$ Acquisition ......................................................... 41

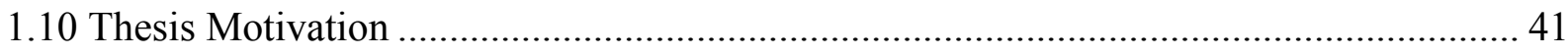

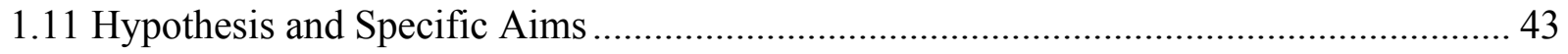

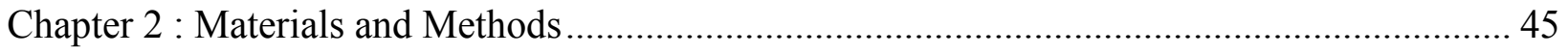

2.1 Blood Products and Storage ........................................................................................ 45

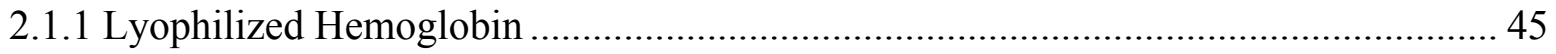

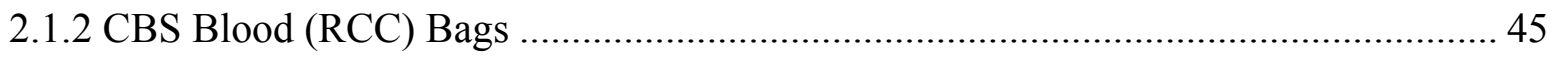

2.2 Radiometer ABL800 FLEX ${ }^{\circledR}$ Blood Gas Analyzer ......................................................... 46

2.2.1 safePICO Aspirator Syringe ................................................................................ 47

2.2.2 Optical System and Spectroscopic Measurement ....................................................... 48

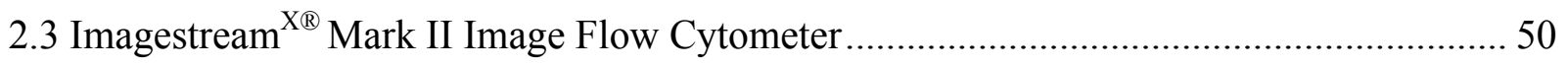

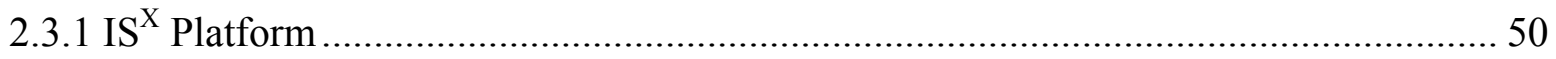

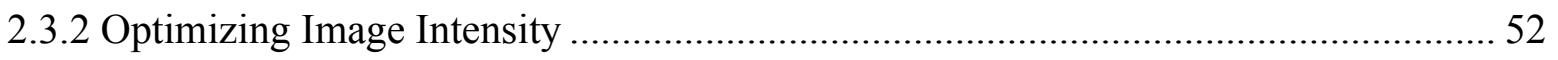

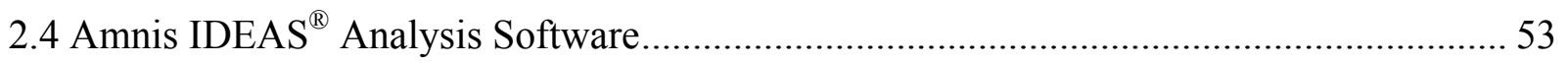

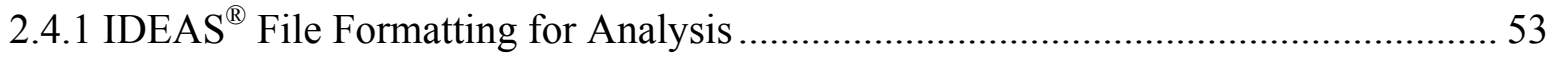

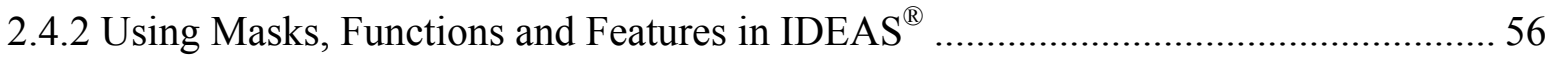

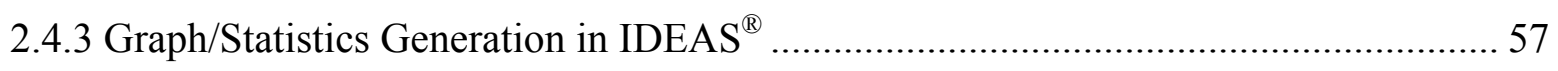

2.4.4 Image Segmentation in IDEAS ${ }^{\circledR}$ : feature finder, truth populations and ROI tracing.. 58

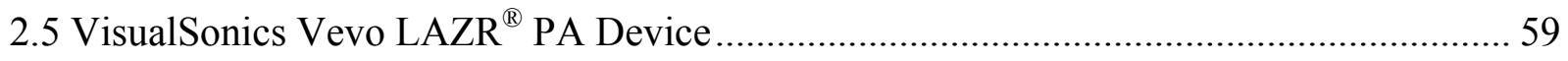

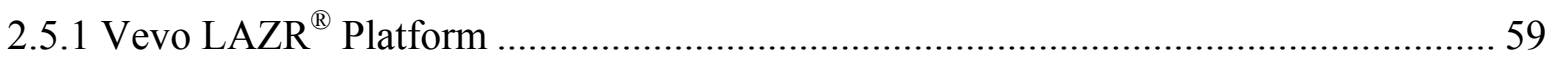

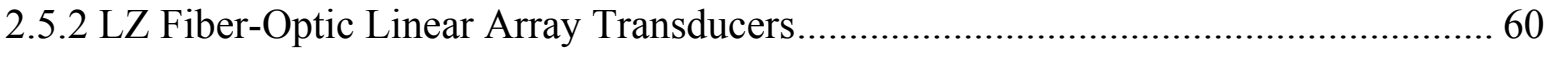




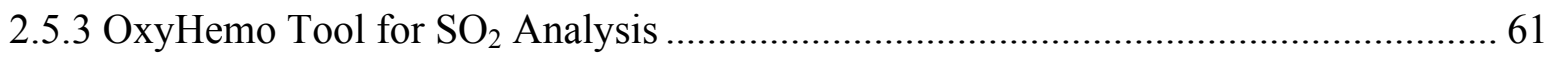

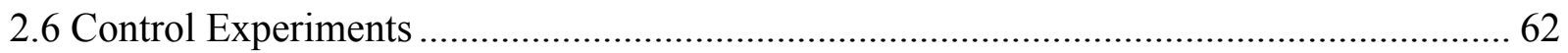

2.6.1 Investigation of Potential PA Signal from Blood Bag Cover ..................................... 62

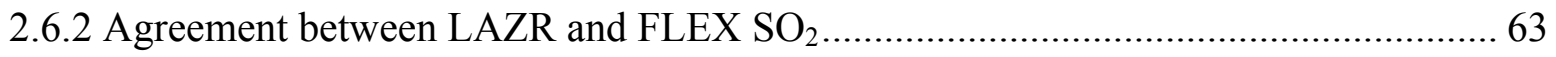

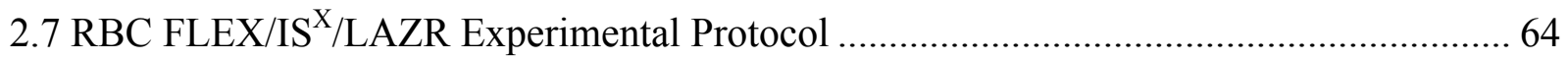

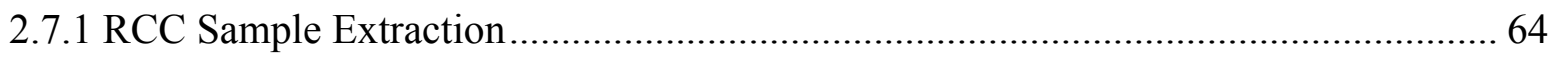

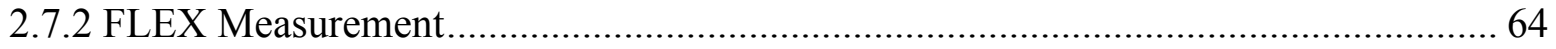

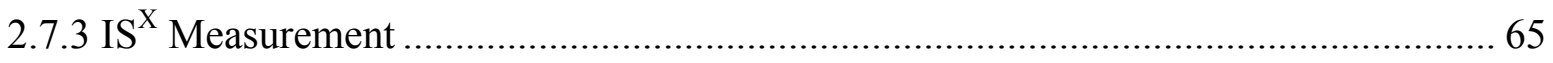

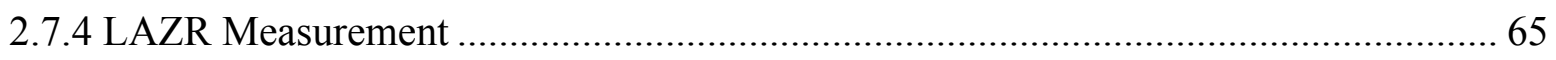

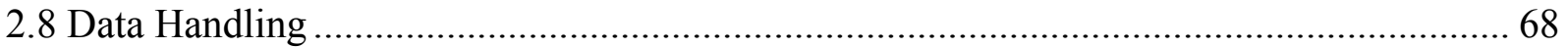

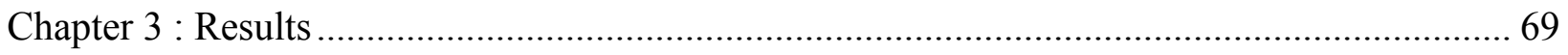

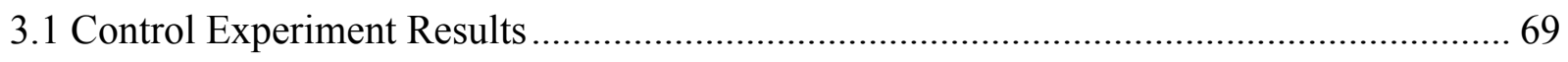

3.2 RBC Morphology Image Segmentation Template ………………………………......... 70

3.3 Spheroechinocyte Percentage Changes versus $\mathrm{SO}_{2}$ Changes in Stored RCCs ................... 78

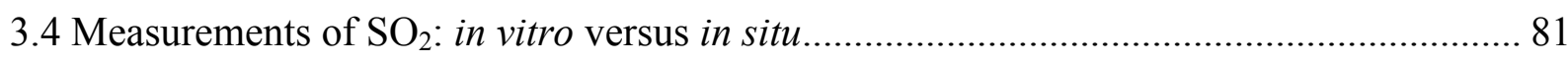

3.5 Changes in RCC Morphology Distribution and in situ $\mathrm{SO}_{2}$ values of Blood Bags............ 84

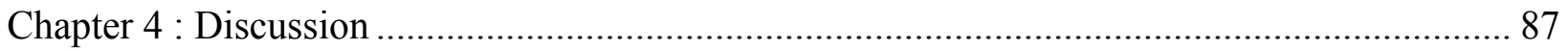

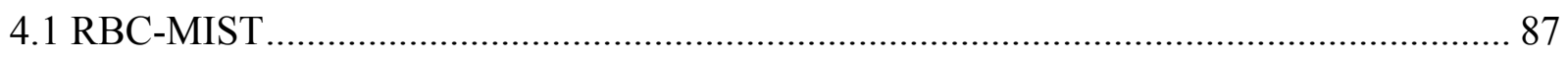

4.2 Spheroechinocyte Percentage Changes versus $\mathrm{SO}_{2}$ Changes in Stored RCCs ................... 90

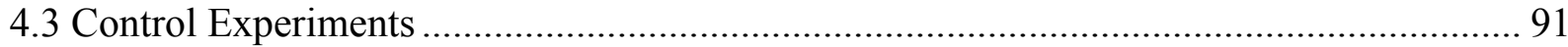

4.4 Measurements of $\mathrm{SO}_{2}$ : in vitro versus in situ ............................................................... 92

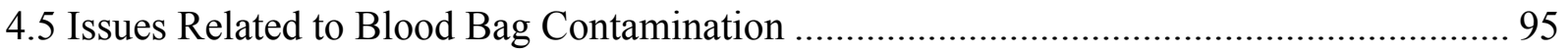

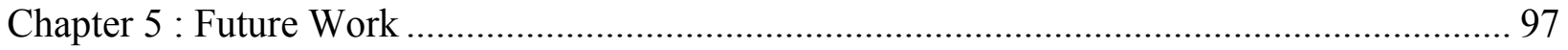

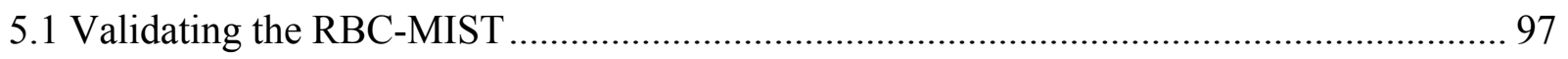

5.2 Validating PA Measurements of Blood Bags in situ .......................................................... 98

5.3 Radio-Frequency Analysis of PA Signals........................................................................ 100

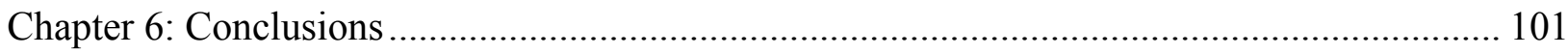

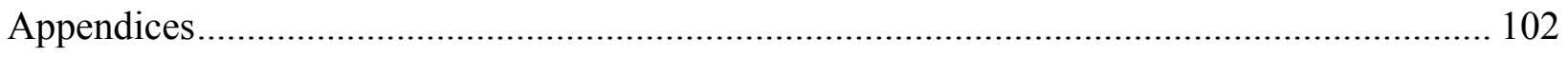

Appendix A: Supplementary Tables and Figures ……………………………………........ 102

Appendix B: Derivation of Absorbance and Molar Extinction Coefficient ............................ 108

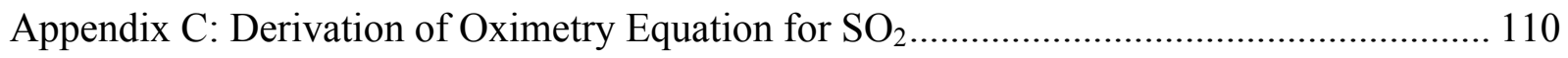




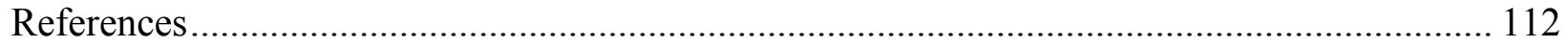




\section{Glossary}

$\begin{array}{ll}\text { 2,3-DPG } & \text { 2,3-diphosphoglycerate } \\ \text { ACD } & \text { Acid-citrate-dextrose } \\ \text { BC } & \text { Buffy coat } \\ \text { BGA } & \text { Blood gas analyser } \\ \text { CBS } & \text { Canadian blood services } \\ \text { COHb } & \text { Carboxyhemoglobin } \\ \text { COI } & \text { Centre of Innovation } \\ \text { CO }_{2} & \text { Carbon dioxide } \\ \text { CPD } & \text { Citrate-phosphate-dextrose } \\ \text { CSTM } & \text { Canadian society for transfusion medicine } \\ \text { deoxyHb } & \text { Deoxyhemoglobin } \\ \text { FDA } & \text { Federal drug administration } \\ \text { Fe } & \text { Iron molecule } \\ \text { FLEX } & \text { Radiometer blood gas analysis system } \\ \text { Hb } & \text { Hemoglobin } \\ \text { HiCN } & \text { Cyanmethemglobin } \\ \text { HQ } & \text { Hèma Québec } \\ \text { ICU } & \text { Intensive care unit } \\ \text { IFC } & \text { Image flow cytometry } \\ \text { IS } & \text { Amnis ImageStream }{ }^{\mathrm{X}} \text { Mk II system } \\ \text { LAZR } & \text { Vevo LAZR photoacoustic system } \\ \text { LTRI } & \text { Lunenfeld-Tanenbaum research insititute } \\ \text { metHb } & \text { Methemoglobin } \\ \text { MI } & \text { Morphology Index } \\ \text { MIST } & \text { Morphology image segmentation template } \\ \text { MSDU } & \text { Multi Spectral Decomposition Unit } \\ \text { ODC } & \text { Oxygen dissociation curve } \\ & \end{array}$




\begin{tabular}{|c|c|}
\hline $\mathrm{O}_{2}$ & Oxygen \\
\hline ORBCoN & Ontario regional blood coordinating network \\
\hline oxyHb & Oxyhemoglobin \\
\hline $\mathrm{P}_{50}$ & $\begin{array}{l}\text { Partial pressure of oxygen that causes } 50 \% \text { oxygen saturation in } \\
\text { hemoglobin }\end{array}$ \\
\hline PA & Photoacoustic \\
\hline PARFS & Photoacoustic radio-frequency spectroscopy \\
\hline PBS & Phosphate buffered saline \\
\hline PLT & Platelet \\
\hline $\mathrm{pO}_{2}$ & Partial pressure of oxygen \\
\hline POC & Point of care \\
\hline $\mathrm{PVC}$ & Polyvinyl chloride \\
\hline $\mathrm{RBC}$ & Red blood cell \\
\hline $\mathrm{RCC}$ & Red cell concentrate \\
\hline $\mathrm{RCT}$ & Random clinical trials \\
\hline $\mathrm{RF}$ & Radio frequency \\
\hline RMS & Root Mean Square \\
\hline ROI & Region of interest \\
\hline SA & Signal amplitude \\
\hline SAGM & Saline-adenine-glucose-mannitol \\
\hline SEM & Scanning electron microscope \\
\hline SNR & Signal to noise ratio \\
\hline $\mathrm{SO}_{2}$ & Oxygen saturation \\
\hline sulfHb & Sulfhemoglobin \\
\hline TDI & Time delay integration \\
\hline US & Ultrasound \\
\hline VIS-NIR & Visible/Near Infrared electromagnetic spectrum \\
\hline WB & Whole blood \\
\hline WBC & White blood cell \\
\hline
\end{tabular}




\section{List of tables}

Table 1-1: List of the cellular components of blood, alternate terminology, in vivo lifespan, percentage blood volume, relative cell number ratio (relative to WBCs) and primary function

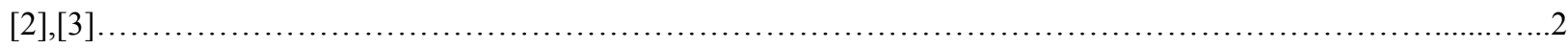

Table 1-2: Categories of RBC storage lesion morphology and their features [83-85] and reversibility [82,86-88], and an example SEM image of each category (adapted from [88]).

Table 2-1: List of the analytes available for measurement using the Radiometer ABL800 FLEX. Table adapted and modified from [126]

Table 2-2: Broadband frequency range axial and lateral resolutions for two LZ ultrasound transducer models [186].

Table 2-3: List of parameters set for US and PA image generation on the LAZR

Table 5-1: General features suggested for segregating pairs of subpopulations provided in each row. Sample images display masked cells. Circularity, spot counts and diameter texture features details provided in [207]; $\mathrm{H}$ textural features published by Haralick et al. [217]. . .98 


\section{List of figures}

Figure 1-1: Diagram of the cardiovascular system; deoxygenated blood is indicated in blue, while oxygenated blood is indicated in red. Adapted from [4]. 3

Figure 1-2: Schematic of an erythrocyte (RBC): (top) cross section and (bottom) aerial view. Adapted from [5].....4

Figure 1-3: Schematic of a regular biconcave disk-shaped RBC; (inset) illustration of hemoglobin, including four globular subunits (red/brown), each containing a heme group bound an oxygen molecule (blue). Adapted from [12].

Figure 1-4: Oxygen Dissociation Curves of hemoglobin with cooperativity (pink) and without cooperativity (blue); percentage values indicate the net $\mathrm{SO}_{2}$ drop between the lungs and tissues via each method. Myoglobin (green) is a heme protein residing primarily in muscle cells, and is shown for comparison only. Adapted from [15]......

Figure 1-5: The right-shift (blue) and left-shift (right) of the Oxygen Dissociation Curve due to various chemical/molecular factors in and around the $\mathrm{RBC}$ environment; $\mathrm{P}_{50}$ is a commonly observed oxygen tension $\left(\mathrm{pO}_{2}\right)$

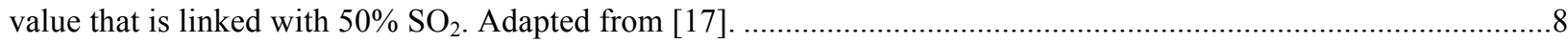

Figure 1-6: (left) BC method of PLT, plasma and RBC extraction; (right) WB method of plasma and RBC extraction. Adapted from [31].

Figure 1-7: Major components of the RBC storage lesion, consequences to the cell and storage medium. Adapted from $[78]$.

Figure 1-8: Intracellular 2,3-DPG concentration (A), as well as changes in supernatant potassium (B), pH (C) and lactate levels (D) as a function of storage duration. Data points are median with 25 and 75 percentile ranges. $P$ values represent significance of change over time. Adapted from [56].

Figure 1-9: $\mathrm{RBC} \mathrm{SO}_{2}$ percentage changes as a function of storage duration. Data points are median with 25 and 75 percentile ranges. $P$ values represent significance of change over time. Adapted from [56]

Figure 1-10: Stored RBC morphology distribution as a function of storage duration; (left) Day 5 of storage is dominated by discocytes; (center) Day 14 of storage is dominated by echinocytes and (right) Day 42 is dominated by irreversible spheroechinocytes. Adapted from [90]

Figure 1-11: Variation in the mean/median of what was considered "fresh" and "old" RCC according to 15 observational studies and 5 RCTs. Adapted from [99].

Figure 1-12: Discrepancy in odds ratios between 31 observational studies and $6 \mathrm{RCTs}$, in determining if fresher or older (regular) RCCs lower mortality rates. Observational studies show an increase in odds of mortality (1.13) when transfusing older blood, while RCTs show no advantage for the transfusion of fresh blood. $\mathrm{I}^{2}$ refers to the statistical measure of heterogeneity. Adjusted refers to confounder adjustments, where applied. Adapted from [99].

Figure 1-13: Absorption coefficients of the two major functional states of hemoglobin (red), plasma proteins (green) and water (blue). Melanin (black) is a major absorbing species in tissue. Adapted from [111].... .26

Figure 1-14: Attenuation of a VIS-NIR beam $I_{0}$ can be attributed to mostly $\mathrm{RBC}$ absorption, under certain circumstances. Derivation of expression of $\mathrm{I}_{1}$ is found in the Appendices $(\mathrm{C})$. Adapted from [115]. .27 
Figure 1-15: Plot of the molar extinction coefficients of oxyHb (indicated by $\mathrm{HbO}_{2}$ ) and deoxyHb (indicated by $\mathrm{Hb}$ ) across the VIS-NIR optical window. Adapted from [117]

Figure 1-16: Shape factor values assigned to morphology features of RBCs (1.5.3); values of $100 \mathrm{RBCs}$ are summed to produce a morphology index (MI). Original method developed by Usry et al. [85].

Figure 1-17: Simplified illustration of the optics (laser/PMT/photodiode), hydraulic (sheath fluid/sample) and .......33

Figure 1-18: (left) An illustration of the subpopulations of WBCs (adapted from [138]); variations in cell size and granularity can be differentiated when (right) producing a dual parameter density plot of forward and side scatter intensities. Adapted from [131]. .35

Figure 1-19: (Left) Plot of Darkfield intensity, or side scatter (SSC), against fluorescein isothiocyante dye (FITC) fluorescence intensity for calibration beads (pink), RBCs (orange), neutrophils (blue), platelets (green) and microvesicles, also known as microparticles (aqua). (Right) Brightfield images from two different CCD camera channels, BODIPY-Maleimide stained fluorescence images, and side scatter images of speed beads and components of whole blood. Images acquired simultaneously using Amnis IS ${ }^{\mathrm{x}}$ IFC. Adapted from [144] .................................37

Figure 2-1: The safePICO Aspirator and components within and encapsulating the syringe..................................48

Figure 2-2: The optics (below) and spectrometer (above) compartments of the ABL800 FLEX BGA. Adapted from [176]. . .49

Figure 2-3: Illustration of the orientation of the flow cell, light sources and microscope objective in the $\operatorname{IS}^{\mathrm{X}}$. Adapted from [179]. . .51

Figure 2-4: Aerial-view diagram outlining the optics platform of the IS ${ }^{\mathrm{X}}$. Note that SSC is an abbreviation for side scatter $785 \mathrm{~nm}$ laser. Adapted from [178]. . .52

Figure 2-5: Illustration of the TDI method of enhancing intensity of image. Each frame consists of (from left to right) the illumination source, cell motion in the sample stream and projection of the corresponding image on a CDD detector. Adapted from [179].

Figure 2-6: Plot of intensity values on a two dimensional grid representing location of pixels on CCD detector. Photometric and Morphometric information is encoded into such plots. Adapted from [178]. . .54

Figure 2-7: Schematic representation of the $\operatorname{IDEAS}^{\circledR}$ file format process flow to obtain a customized template for automated analysis.

Figure 2-8: Layout of a daf file created by a template file geared for the analysis of RBCs. Typical layouts include the image gallery (left) and the analysis area (right) that generates graphs and associated statistics. .. .55

Figure 2-9: Example of the user interface for creating a new, custom mask on the IDEAS ${ }^{\circledR}$ mask manager; all default masks and created functions are available for manipulation with Boolean commands. The mask is saved and can be applied on any image. Adapted from [182]. .556

Figure 2-10: Example of selecting multiple features for the analysis of channel 4 images; mask M4 was chosen for ROI detection. Adapted from [182]. .57

Figure 2-11: Example of selecting multiple parameters in the statistics area. Adapted from [182]. .58

Figure 2-12: The Vevo LAZR Photoacoustic imaging system and the major system components. Adapted from [185]. . .59 
Figure 2-13: (left) Illustration of LZ ultrasound transducer encasement and (right) orientation of optic and acoustic sources at tip of the transducer. Adapted from [188].

Figure 2-14: (left) Illustration of a lateral view of the LZ light source geometry and (right) axial view of the optical (red) and ultrasound (yellow) beam geometry. Adapted from [188]. 61

Figure 2-15: Vevo LAZR PA $\mathrm{SO}_{2}$ map overlaid on a coregistered B-mode US image of a Lewis lung carcinoma in a mouse flank. Green/yellow ROIs manually picked; $\mathrm{sO}_{2}$ Avr represents the average percentage $\mathrm{SO}_{2}$ value calculated for the ROI. Adapted from [188].

Figure 2-16: A cylindrical glass container, covered with blood bag plastic and filled with (left) water and (right) blood; the extensions used to inject the medium were sealed with vaseline.

Figure 2-17: (left) Side view and (right) Ariel view of a schematic representation of the blood bag LAZR container. Illustration courtesy of K. Bagga.

Figure 2-18: $\mathrm{PA} \mathrm{SO}_{2}$ map of a blood bag, including the traced region (purple circle) for $\mathrm{SO}_{2}$ calculation (red box). For increasing $\mathrm{SO}_{2}$, scale ranges from dark red to light pink. Background black/white signal represents co-registered US image. Yellow arrow represents the optical focal depth $(11 \mathrm{~mm})$. Inset displays setup used for measurement. ....68

Figure 3-1: Absolute value of the average RF signal (11 lines) of water (blue) and oxygenated ( 85\%) RCC (red) in a glass container covered with $0.35 \mathrm{~mm}$ thick blood bag plastic (dashed lines), whose top boundary is located at $10 \mathrm{~mm}$.

Figure 3-2: FLEX (blue), LAZR LZ250 (red) and LAZR LZ550 (green) mean $\mathrm{SO}_{2}$ values of heavily deoxygenated (1), evenly oxygenated (2) and heavily oxygenated (3) lyophilized hemoglobin. Standard deviation bars represent 3 independent FLEX measurements, and 10 frames of 1 LAZR measurement. .70

Figure 3-3: (Top Row) Typical RBC images produced by the IS ${ }^{\mathrm{X}}$ IFC system, including unfocused (A), multi-cell (B), cells with calibration beads (C), front view discocytes (D, E), side view discocytes (F), side view spheroechinocytes $(\mathrm{G})$ and front view spheroechinocytes (H, I). (Second Row and Below) Sequential gating based on various mask/feature combinations was used to exclude objects of type A (I.), B (II.), C (III.), and differentiate populations D-F from G-J to classify side view and front view images, respectively. .71

Figure 3-4: (Top Row) Typical RBC images that include smooth/crenated discs (K,L), crenated discoid (M), crenated spheroid $(\mathrm{N})$, smooth/crenated spheres (O,P), side view spheroechinocytes (Q), and side view discocytes (R). (Second Row and Below) Sequential gating based on various mask/feature combinations (V. - VIII.) was used to differentiate populations $\mathrm{K}, \mathrm{L}, \mathrm{M}$ and $\mathrm{R}$ (curved red box) from N, O, P and Q (right angled red box) to classify discocytes and spheroechinocytes, respectively. .72

Figure 3-5: (see step I, Figure 3-3); histogram of gradient RMS (counts of normalized relative frequency) within the default bright field mask. Inset images provide examples of unfocused (left) and focused (right) RBC images. Object masks are highlighted in light blue. Value of gradient RMS of images are indicated by red arrows. Images containing gradient RMS values above 54 were selected for further analysis (horizontal light blue line).

Figure 3-6: (see step II, Figure 3-3); plot of aspect ratio versus area using the default bright field mask. Inset images provide examples of front view (top right), multi-cell (middle right) and side view (bottom left) RBC images. Object 
masks are highlighted in light blue. Red arrows point to the location of images within the scatter plot. A gate based on images within the ROI (light green boundary) was selected for further analysis.

Figure 3-7: (see step III, Figure 3-3); plot of spot count using two variations of the erode mask. Inset images provide examples of cells with calibration beads (top right) and single cell (bottom left) RBC images. Object masks are highlighted in light blue. Red arrows point to the location of images within the scatter plot. Only images with a spot count of 1 (for both masks) were selected for further analysis.

Figure 3-8: (see step IV, Figure 3-3); plot of circularity versus aspect ratio, using the watershed mask with an adaptive erode function. Inset images provide examples of front view (top) and side view (bottom) RBC images. Object masks are highlighted in light blue. Red arrows point to the location of images within the scatter plot. A gate based on images within both ROIs (green/blue boundary) were selected for further analysis. . .75

Figure 3-9: (see step V, Figure 3-4); plot of Symmetry versus H-variance, using the watershed mask. Inset images provide examples of side view discocyte (top) and side view spheroechinocyte (bottom) RBC images. Object masks are highlighted in light blue. Red arrows point to the location of images within the scatter plot. Feature finder/Truth populations were used to determine best features; overlaid on the sample population are truth populations for spheroechinocytes (purple) and discocytes (pink). A gate based on images within both ROIs (pink/grey boundary) were selected for analysis. . .76

Figure 3-10: (see step VI, Figure 3-4); plot of Gradient RMS for a watershed mask versus a watershed AND NOT erode mask. Inset images provide examples of brighter shaded (left) and darker shaded (right) RBC images. Object masks are highlighted in light blue. Red arrows point to the location of images within the scatter plot. A gate based on images within both ROIs (yellow/orange boundary) were selected for analysis. . .77

Figure 3-11: (see step VII, Figure 3-4); plot of Contrast versus H-Variance, using the watershed mask. Inset images provide examples darker shaded spheroechinocyte (top left) and discocyte (bottom right) RBC images. Object masks are highlighted in light blue. Red arrows point to the location of images within the scatter plot. Feature finder/Truth populations were used to determine best features; truth populations not overlaid in figure for simplicity. A gate based on images within both ROIs (red/blue boundary) were selected for analysis.

Figure 3-12: (see step VIII, Figure 3-4); plot of H correlation standard deviation versus H-correlation mean, using the watershed mask. Inset images provide examples of brighter shaded spheroechinocyte (left) and discocyte (right) RBC images. Object masks are highlighted in light blue. Red arrows point to the location of images within the scatter plot. Feature finder/Truth populations were used to determine best features; truth populations not overlaid in figure for simplicity. A gate based on images within both ROIs (pink/beige boundary) were selected for analysis...78 Figure 3-13: Plot of FLEX $\mathrm{SO}_{2}$ (black) and IS ${ }^{\mathrm{X}}$ Spheroechinocyte population percentage (red) mean values for RCC samples of Bags A,B,D,E-H over their 6 week lifespan. Bars indicate standard deviation of values obtained from three samples.... . .79

Figure 3-14: Plot of mean percentage changes of $\mathrm{FLEX} \mathrm{SO}_{2}$ (black) and IS ${ }^{\mathrm{X}}$ Spheroechinocyte population (red) for RCC samples of Bags A,B,D,E-H over their 6 week lifespan. Changes are calculated with respect to initial (Day 9) measurement. Bars indicate (error-propagation accounted) standard deviation of values obtained from three samples. $r^{2}$ values calculated as the square of correlation coefficient. . .80 
Figure 3-15: Bland-Altman plot of $\mathrm{SO}_{2}$ and Spheroechinocyte population percentage change including Bags A,B,D,E-H over their 6 week lifespan.

Figure 3-16: Plot of LAZR in situ $\mathrm{SO}_{2}$, for LZ250 (solid blue) and LZ550 (dashed blue) transducers, against FLEX $\mathrm{SO}_{2}$ (black) $\mathrm{SO}_{2}$ mean values for extracted RCC samples and Bags A,B,D,E-H over their 6 week lifespan. Bars indicate standard deviation of values obtained from three ROIs/samples. $r^{2}$ values calculated as the square of correlation coefficient.

Figure 3-17: Bland-Altman plot of in situ LAZR LZ250 and in vitro FLEX $\mathrm{SO}_{2}$ measurements for Bags A,B,D,E-H over their 6 week lifespan. .83

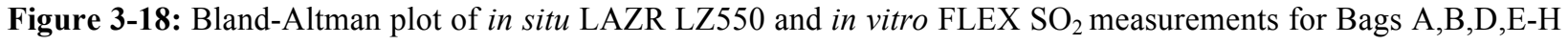
over their 6 week lifespan.

Figure 3-19: Bland-Altman plot of in situ LAZR LZ250 and LAZR LZ550 measurements for Bags A,B,D,E-H over their 6 week lifespan. .84

Figure 3-20: Plot of LAZR in situ $\mathrm{SO}_{2}$ changes for LZ250 (red) and LZ550 (blue) transducers, against ISX spheroechinocyte population changes (black) for extracted RCC samples and Bags A,B,D,E-H over their 6 week lifespan. FLEX $\mathrm{SO}_{2}$ changes (green dashed) superimposed for comparison. Bars indicate standard deviation of values obtained from three ROIs/samples. $\mathrm{r}^{2}$ values calculated as the square of correlation coefficient. .85

Figure 3-21: Bland-Altman plot of in situ LAZR LZ250 $\mathrm{SO}_{2}$ changes and in vitro ISX spheroechinocyte population percent changes for Bags A,B,D,E-H over their 6 week lifespan.

Figure 3-22: Bland-Altman plot of in situ LAZR LZ550 $\mathrm{SO}_{2}$ changes and in vitro ISX spheroechinocyte population percent changes for Bags A,B,D,E-H over their 6 week lifespan. .86

Figure 4-1: Setup developed to measure the PA SO2 value of extracted RCC samples from safePICO syringes, immediately after use in the FLEX.

Figure 4-2: Syringe and in situ blood bag $\mathrm{SO}_{2}$ measurements acquired by the LAZR LZ250 (21MHz) against FLEX $\mathrm{SO}_{2}$ values. Bags of varying oxygenation were used to investigate range of agreement. Dashed line refers to theoretical perfect agreement between BGA and PA. X/Y axis error bars refer to mean and standard deviation of three measurements (3 syringes/3 ROIs) per blood bag.

Figure 4-3: Syringe and in situ blood bag $\mathrm{SO}_{2}$ measurements acquired by the LAZR LZ550 (40MHz) against FLEX $\mathrm{SO}_{2}$ values. Bags of varying oxygenation were used to investigate range of agreement. Dashed line refers to theoretical perfect agreement between BGA and PA. X/Y axis error bars refer to mean and standard deviation of

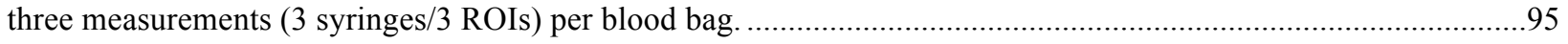

Figure 4-4: Abrupt declines in FLEX and LAZR in situ $\mathrm{SO}_{2}$ values for bags $\mathrm{D}, \mathrm{G}$ and $\mathrm{H}$ .96

Figure 5-1: Derived absorption spectra acquired from DR measurements of three blood bags in situ. The spectral behavior in this region provides an indication of the oxygenation of the blood bags. 100 


\section{List of appendices}

Table A-1: List of some specification of the safePICO Aspirator syringe provided by Radiometer [200] 102

Table A-2: Spectral Features of $\mathrm{IS}^{\mathrm{X}}$, including channel bandwidths, applicable dyes for available lasers. Not included is $830 \mathrm{~nm}$ laser used to scatter light from beads in sheath flow for detection in autofocus/flow speed system. Adapted from [204]. .102

Table A-3: Names, brief descriptions and parameters of the masks available on IDEAS ${ }^{\circledR}$ [207]. 103

Table A-4: Names and brief descriptions of the features available on IDEAS $^{\circledR}$ [207]. 104

Figure A-1: Picture of a blood bag fitted with a sampling site coupler, with a needle inserted into the coupler for RCC extraction. 105

Figure A-2: Picture displaying the safeTIPCAP driving air out of the sample while sealing the sample within the syringe 105

Figure A-3: Picture of three samples extracted from one blood bag, ready for transport to FLEX. .105

Figure A-4: Picture of three samples placed in the FLEX device for automated analysis. 106

Figure A-5: Picture of (left) the $\mathrm{IS}^{\mathrm{X}}$ device, as well as (right) the sample holder area for vial placement. Images adapted from [205].

Figure A-6: Setup of the LAZR laser calibration, to correct for differences between the LAZR cart energy values and the transducer output energy values. .

Figure A-7: Positioning of blood bag LAZR container within the LAZRTight container. .107

Figure A-8: blood bag LAZR setup: (top left) hanging of bag to separate air pockets; (top right) folding of coupler and tubing; (bottom left) gradual angled sliding of bag onto the container bed and; (bottom right) simultaneous introduction of container lid for horizontal flattening and stability of bag.

Figure A-9: (left) removing of air bubbles after insertion into water and (right) final position of transducer over blood bag for $\mathrm{PA} \mathrm{SO}_{2}$ measurements

Figure B-10: Schematic representation outlining the key variables used in the derivation of Beer's law. Photons of intensity $I_{0}$ traverse through a slab of thickness $d x$, after which the intensity decreases by an amount $d I$ due to absorbing/scattering species in the slab. Adapted from [196]. 


\section{Chapter 1 : Introduction}

The introduction chapter is composed of three major themes: red blood cells (RBCs), storage lesions and optical techniques. This chapter aims to provide the reader with 1) the relevant definitions and properties of each theme; 2) a brief overview of the pertinent literature behind each theme and 3) the connection between each theme in forming the motivation behind this thesis project.

In addressing the first theme, Section 1.1.1 briefly introduces the role and constituents of blood before presenting the structural properties of RBCs in Section 1.1.2. In Section 1.2, the functional properties of RBCs are discussed in the context of its primary constituent hemoglobin. Section 1.3 briefly introduces the field of RBC transfusion, touching on its significance within the health care field.

For the second theme, Section 1.4 provides an account of the guidelines for pre-transfusion RBC storage. Section 1.5 defines the characteristics of an RBC storage lesion, its negative effects on stored RBCs and possible clinical consequences of transfusing damaged RBCs.

The third theme is presented with a brief introduction to the optical properties and techniques used to monitor RBCs (Section 1.6). Section 1.7 showcases some conventional lab methods employing the presented optical techniques to study various properties of the RBC storage lesion. Sections 1.8 and 1.9 present two relatively novel optical technologies, image flow cytometry (IFC) and photoacoustics (PA); furthermore, these sections present the potential of these technologies to monitor damaged RBCs during storage in novel ways.

Section 1.10 merges the three major themes to form the thesis motivation: the optical technologies of image flow cytometry and photoacoustics have unique capabilities of monitoring certain effects on stored RBCs due to the storage lesions. The hypotheses presented in Section 1.11. are concerned with the ability of each technology to measure a particular characteristic of the RBC storage lesion. 


\subsection{Red Blood Cells: An Introduction}

\subsubsection{Blood}

Blood, a connective tissue in the body [1], contains several cellular components that are suspended in the extracellular fluid matrix called plasma. Plasma occupies approximately $55 \%$ of the total blood volume. It is primarily composed of water and contains a suspension of proteins, electrolytes and antibodies, amongst other molecules [2]. Table 1-1 presents information on the cellular components of blood:

\begin{tabular}{|c|c|c|c|c|c|}
\hline $\begin{array}{c}\text { CELL } \\
\text { NAME }\end{array}$ & $\begin{array}{c}\text { ALTERNATE } \\
\text { TERMINOLOGY }\end{array}$ & $\begin{array}{c}\text { IN-VIVO } \\
\text { LIFESPAN } \\
\text { (DAYS) }\end{array}$ & $\begin{array}{c}\text { (\%) BLOOD } \\
\text { VOLUME }\end{array}$ & $\begin{array}{c}\text { RELATIVE } \\
\text { CELL RATIO }\end{array}$ & $\begin{array}{c}\text { PRIMARY } \\
\text { FUNCTION }\end{array}$ \\
\hline $\begin{array}{c}\text { White } \\
\text { Blood } \\
\text { Cell } \\
\text { (WBC) }\end{array}$ & Leukocyte & $3-4$ & $<1$ & 1 & $\begin{array}{c}\text { Defense against } \\
\text { harmful } \\
\text { endo/exogenous } \\
\text { agents }\end{array}$ \\
\hline $\begin{array}{c}\text { Platelet } \\
\text { Thrombocyte }\end{array}$ & $5-9$ & $<1$ & 40 & $\begin{array}{c}\text { Prevention of blood } \\
\text { leakage via clotting }\end{array}$ \\
\hline $\begin{array}{c}\text { Red } \\
\text { Cell } \\
\text { RBC) }\end{array}$ & Erythrocyte & $100-120$ & $40-45$ & 650 & $\begin{array}{c}\text { Oxygen } \\
\text { Transport/Delivery }\end{array}$ \\
\hline
\end{tabular}

Table 1-1: List of the cellular components of blood, alternate terminology, in vivo lifespan, percentage blood volume, relative cell number ratio (relative to WBCs) and primary function [2],[3]

Blood traverses through the human body via the cardiovascular system (Figure 1-1). Deoxygenated blood is pumped from the heart to the lungs, where it is exposed to partial pressures that induce the release of carbon dioxide $\left(\mathrm{CO}_{2}\right)$ and the uptake of oxygen $\left(\mathrm{O}_{2}\right)$. The freshly oxygenated blood then returns to the heart, before being pumped out to the rest of the body via the aorta. The diffusion of blood gases within the body occurs primarily in the microvasculature. 


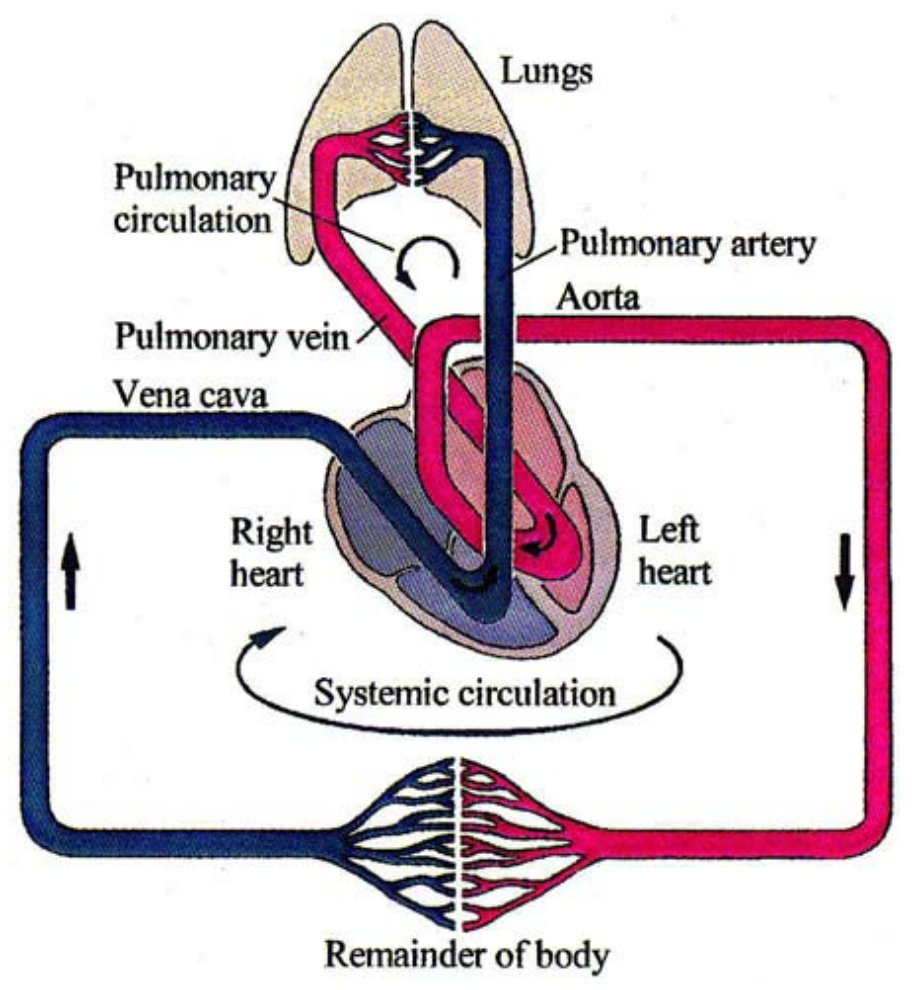

Figure 1-1: Diagram of the cardiovascular system; deoxygenated blood is indicated in blue, while oxygenated blood is indicated in red. Adapted from [4].

\subsubsection{Red Blood Cells (RBCs)}

Red blood cells (RBCs) are the most prevalent cellular constituents of blood, both in relative cell quantity and in percentage volume occupied in blood (Table 1-1). A mature, normal functioning $\mathrm{RBC}$ is anucleated and has a biconcave disc morphology. It has a diameter of approximately $7.5 \mu \mathrm{m}$ and thickness ranging between 1.5 to $2.5 \mu \mathrm{m}$ (Figure 1-2) [5, 6]. The RBC cell membrane is composed of a phospholipid bilayer, an internal cytoskeletal network and membrane proteins. The membrane and cytoskeleton help maintain the biconcave shape [7].

The biconcave shape of RBCs sustains laminar flow more effectively than other shapes with similar surface-to-volume ratios - this reduces damage to the vasculature and lowers the demand on the heart for maintaining consistent blood flow [8]. The elastic nature of the RBC membrane and cytoskeleton facilitates the transportation of blood gases within the human body. RBCs have 

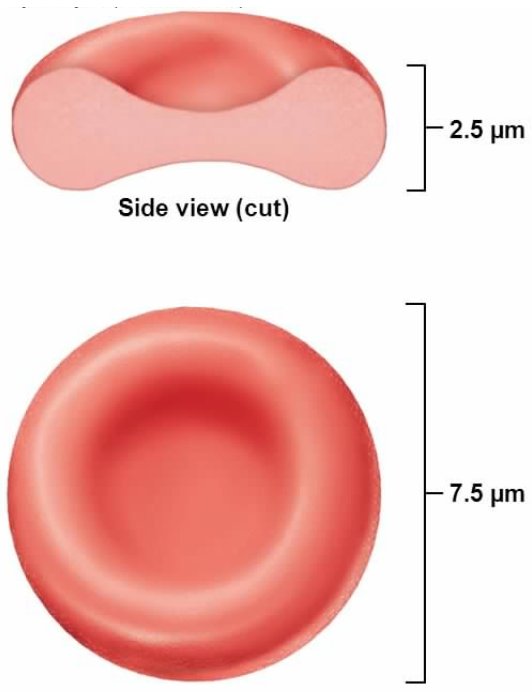

Figure 1-2: Schematic of an erythrocyte (RBC): (top) cross section and (bottom) aerial view. Adapted from [5].

a deformable membrane that allows them to compress and squeeze through microvasculature [9], and to recover their original biconcave shape upon exiting [10].

Deviations in RBC membrane composition and morphology can result in unfavorable consequences. For instance, a decrease in $\mathrm{RBC}$ deformability can lead to an inability to enter the microvasculature, which can potentially lead to necrosis and organ failure [8]. Hence, knowledge of certain functional and morphological parameters of RBCs could provide vital information about the physiological impact transfused RBCs can have in the body.

\subsection{Hemoglobin: An Introduction}

\subsubsection{Hemoglobin Structure}

RBCs have the critical task of delivering $\mathrm{O}_{2}$ to - and to a lesser extent removing $\mathrm{CO}_{2}$ from - every part of the body. Consequently, the major molecular constituent in the RBC cytosol is hemoglobin, the $\mathrm{O}_{2} / \mathrm{CO}_{2}$ carrier protein. An $\mathrm{RBC}$ typically contains approximately 270 million molecules of hemoglobin [11]. If this hemoglobin content were to exist freely in plasma, the consistency of the blood would dramatically increase in viscosity, causing more turbulent blood 
flow [8]. Hence, hemoglobin is compartmentalized within RBCs to maximize hemoglobin content in blood while maintaining hemodynamic stability.

The inset of Figure 1-3 provides an illustration of the structure of hemoglobin [12]. Hemoglobin is a globular protein that consists of four polypeptide chains (globular protein subunits). Each subunit contains a heme group with a central iron atom that binds to $\mathrm{O}_{2}$ reverisbly [13].

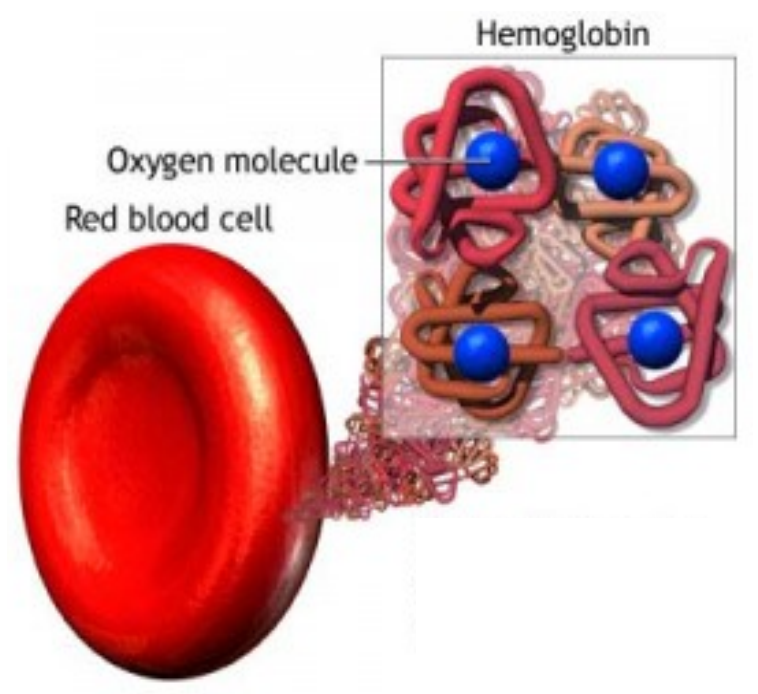

Figure 1-3: Schematic of a regular biconcave disk-shaped RBC; (inset) illustration of hemoglobin, including four globular subunits (red/brown), each containing a heme group bound an oxygen molecule (blue). Adapted from [12].

\subsubsection{Hemoglobin States}

When all four heme irons in hemoglobin are in their reduced ( +2 oxidation) state and are unbound to any ligand, the hemoglobin molecule is termed as deoxyhemoglobin (deoxyHb). Each iron molecule in deoxyHb can bind reversibly with oxygen [13]:

$$
\mathrm{Fe}(\mathrm{II})+\mathrm{O}_{2} \rightleftharpoons \mathrm{Fe}(\mathrm{II}) \mathrm{O}_{2}
$$

where Fe(II), represents the ferrous (reduced) state of iron; the right-hand side of Equation 1-1 represents the binding of oxygen, turning deoxyHb into oxyhemoglobin (oxyHb). 
Occasionally, the equilibrium presented in Equation 1-1 can deviate. Approximately 3\% of ferrous iron converts into a ferric ( +3 oxidation) state [13]. This spontaneous auto-oxidation equilibrium is expressed as:

$$
\mathrm{Fe}(\mathrm{II})+\mathrm{O}_{2} \rightleftharpoons \mathrm{Fe}(\mathrm{III})+\mathrm{O}_{2}^{-}
$$

Hemoglobin with a ferric iron base is termed methemoglobin (metHb). The oxidation of iron in metHb results in a conformational change in the heme group that makes it unable to bind to any ligand. RBCs contain reductases that reduce a majority of ferric iron atoms back to the ferrous state. Subsequently, the net metHb that exists in RBCs in vivo does not exceed 3\% [11]. MetHb falls under a broader category of alternate conformations, termed dyshemoglobins, that result in an inability to bind to oxygen or carbon dioxide; examples include carboxyhemoglobin $(\mathrm{COHb})$ and sulfhemoglobin (sulfHb) which, like metHb, exist in minute quantities in a healthy individual [14].

\subsubsection{Oxygen Saturation, Oxygen Dissociation Curve and Cooperative Binding}

The binding of oxygen to hemoglobin is an allosteric process, such that the hemoglobin molecule undergoes conformational changes as each heme group binds to an oxygen molecule. Furthermore, the conformational changes augment the binding potential of the unbound heme groups to oxygen [13]. Termed as cooperative binding, the conformational changes provide an enhancement to the $\mathrm{O}_{2}$ carrying capacity of hemoglobin [15].

Oxygen saturation $\left(\mathrm{SO}_{2}\right)$, is an important clinical parameter that indicates the percentage of hemoglobin saturated with oxygen, and is given by [14],

$$
\mathrm{SO}_{2}=\frac{\mathrm{oxyHb}}{\mathrm{oxyHb}+\operatorname{deoxyHb}} \times 100 \% \text {, }
$$

where it is assumed that any forms of hemoglobin, other than oxyHb and deoxyHb, can be neglected. Plotting $\mathrm{SO}_{2}$ as a function of increasing $\mathrm{pO}_{2}$, known as the oxygen dissociation curve (ODC), generates a sigmoid shape that illustrates cooperative binding. Figure 1-4 displays the 
ODC curve for hemoglobin with and without cooperativity [15]. Both avenues require the same amount of $\mathrm{pO}_{2}$ to bind to one oxygen molecule and reach $25 \% \mathrm{SO}_{2}$, after which $\mathrm{pO}_{2}$ dependence differs drastically. Cooperative binding requires roughly 1.5 times the initial $\mathrm{pO}_{2}$ to bind to the second oxygen molecule $\left(50 \% \quad \mathrm{SO}_{2}\right)$, while 3 times the initial $\mathrm{pO}_{2}$ is needed without cooperativity. These differences progressively increase with increasing $\mathrm{SO}_{2}$. The figure also illustrates that, as a result of cooperative binding, 1.7 times more oxygen is released to the tissues [15].

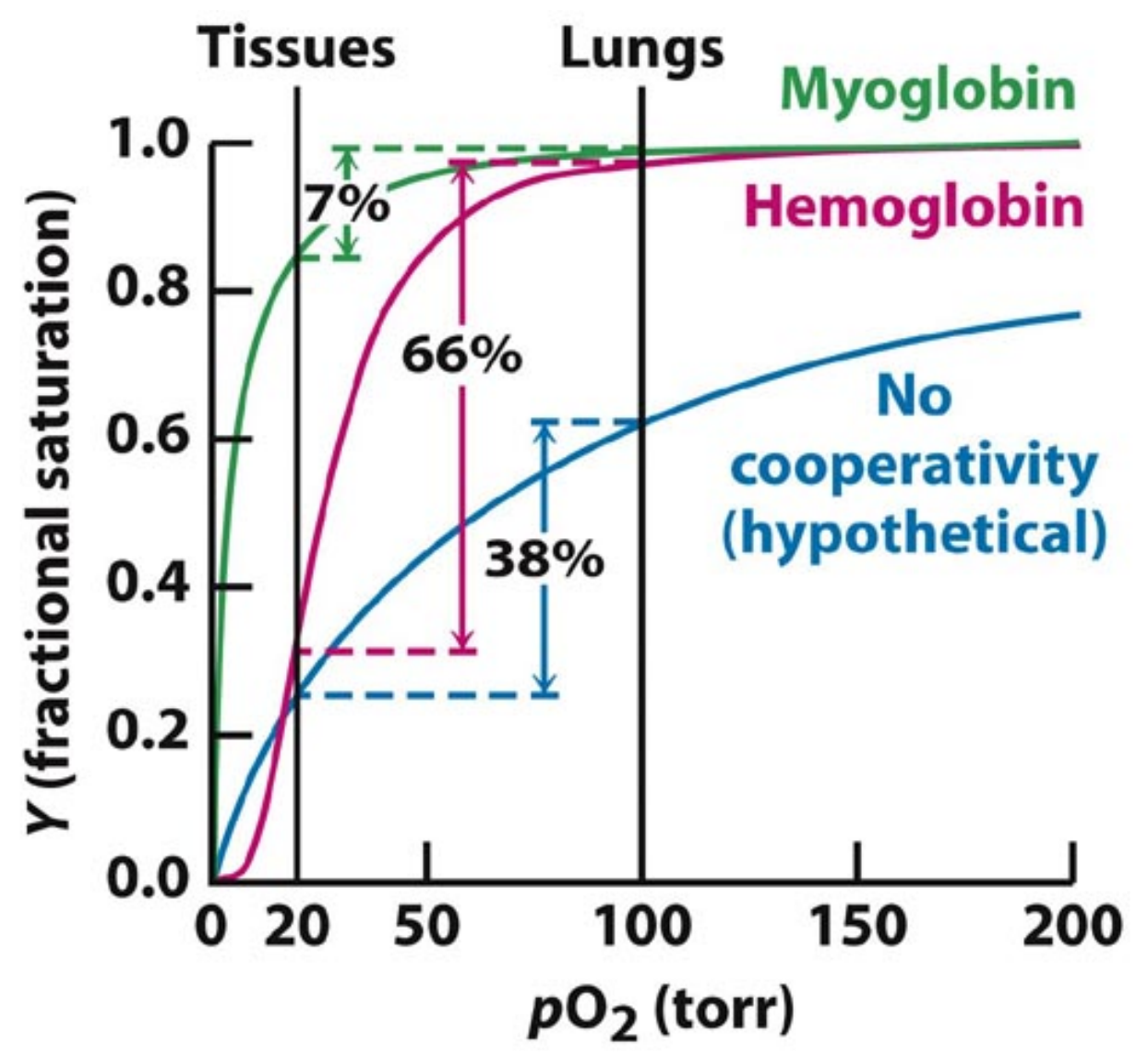

Figure 1-4: Oxygen Dissociation Curves of hemoglobin with cooperativity (pink) and without cooperativity (blue); percentage values indicate the net $\mathrm{SO}_{2}$ drop between the lungs and tissues via each method. Myoglobin (green) is a heme protein residing primarily in muscle cells, and is shown for comparison only. Adapted from [15].

\subsubsection{Factors affecting the ODC}

The oxygen binding capacity of hemoglobin can be affected by various chemical factors within the $\mathrm{RBC}$ and surrounding environment. A decrease in $\mathrm{pH}$ levels and/or an increase in blood temperature or $\mathrm{CO}_{2}$ partial pressure results in a decrease in $\mathrm{SO}_{2}$ for any given $\mathrm{pO}_{2}$ (the $\mathrm{Bohr}$ 
effect) [16]. In other words, these chemical changes produce a shift of the ODC curve, which is illustrated in Figure 1-5 [17].

Unlike the factors mentioned thus far, 2,3-diphosphoglycerate (2,3-DPG) regulates the affinity of hemoglobin to oxygen directly [18]. In RBCs, the concentration of 2,3-DPG is nearly equimolar to that of hemoglobin [19]. By favorably binding to deoxyHb, it significantly enhances the net release of oxygen into the surrounding environment [19].

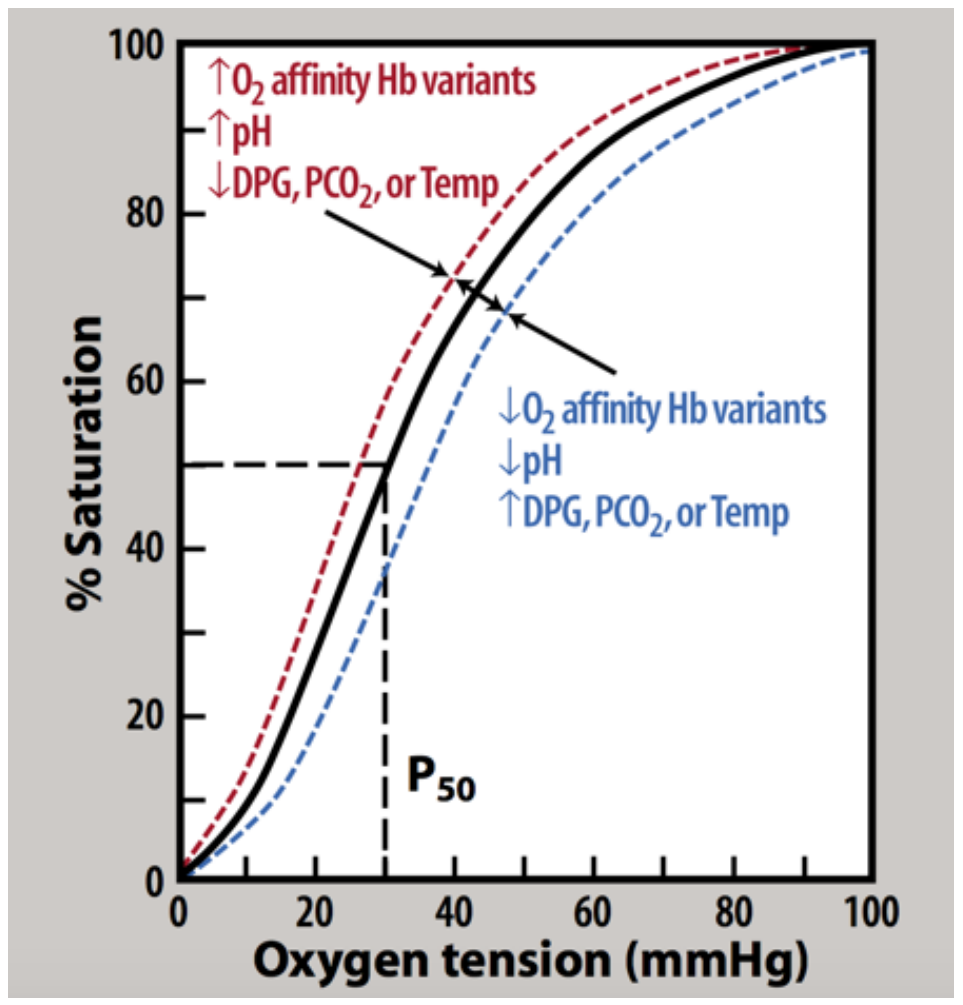

Figure 1-5: The right-shift (blue) and left-shift (right) of the Oxygen Dissociation Curve due to various chemical/molecular factors in and around the $\mathrm{RBC}$ environment; $\mathrm{P}_{50}$ is a commonly observed oxygen tension ( $\mathrm{pO}_{2}$ ) value that is linked with $50 \% \mathrm{SO}_{2}$. Adapted from [17].

\subsection{Transfusion Medicine}

Transfusion medicine, or Transfusiology, is a branch of hematology that is focused on blood conservation and the transfusion of blood components [20]. Each blood component collected for transfusion has unique collection methods, storage requirements and transfusion-related limitations. Approximately 85 million RBC units are transfused annually worldwide, however, 
varying transfusion practices result in different standards of testing, storing and transfusion protocols from nation to nation [21,22]. A lack of globally unified guidelines in blood transfusion is partially attributed to a dearth of concrete evidence on the benefits and risks associated with transfusion practices $[23,24]$. This section briefly discusses the regulation of transfusion medicine in Canada and the collection methods of RBCs.

\subsubsection{Canadian Transfusion Management}

Canada relies on government-affiliated organizations to enforce guidelines and manage the supply and demand of transfusions. Health Canada regulates hospital transfusion practices by providing published guidelines that are approved by the Canadian Society for Transfusion Medicine (CSTM) [25]. Along with these federal guidelines, provincial bodies like the Ontario Regional Blood Coordinating Network (ORBCoN) also contribute to the CSTM's resource database [26]. Hèma Québec (HQ) and Canadian Blood Services (CBS) are the two agencies responsible for the collection, storage and transportation of blood in Québec and all other provinces, respectively.

\subsubsection{RBC Collection and Storage}

At CBS donation clinics/centers, approximately one pint of blood is collected directly from the donor into a polyvinyl chloride (PVC) steam-sterilized bag that contains $70 \mathrm{ml}$ of citratephosphate-dextrose (CPD) anti-coagulant solution [27]. The bag is then processed through either the buffy coat (BC) filtration method, or the whole blood (WB) filtration method (Figure 1-6). In the $\mathrm{BC}$ method, donated blood is first centrifuged to separate RBCs from the buffy coat and plasma; the RBCs are transferred into another PVC bag containing $110 \mathrm{ml}$ of Saline-AdenineGlucose-Mannitol (SAGM) additive solution, and is then removed of WBCs (termed 


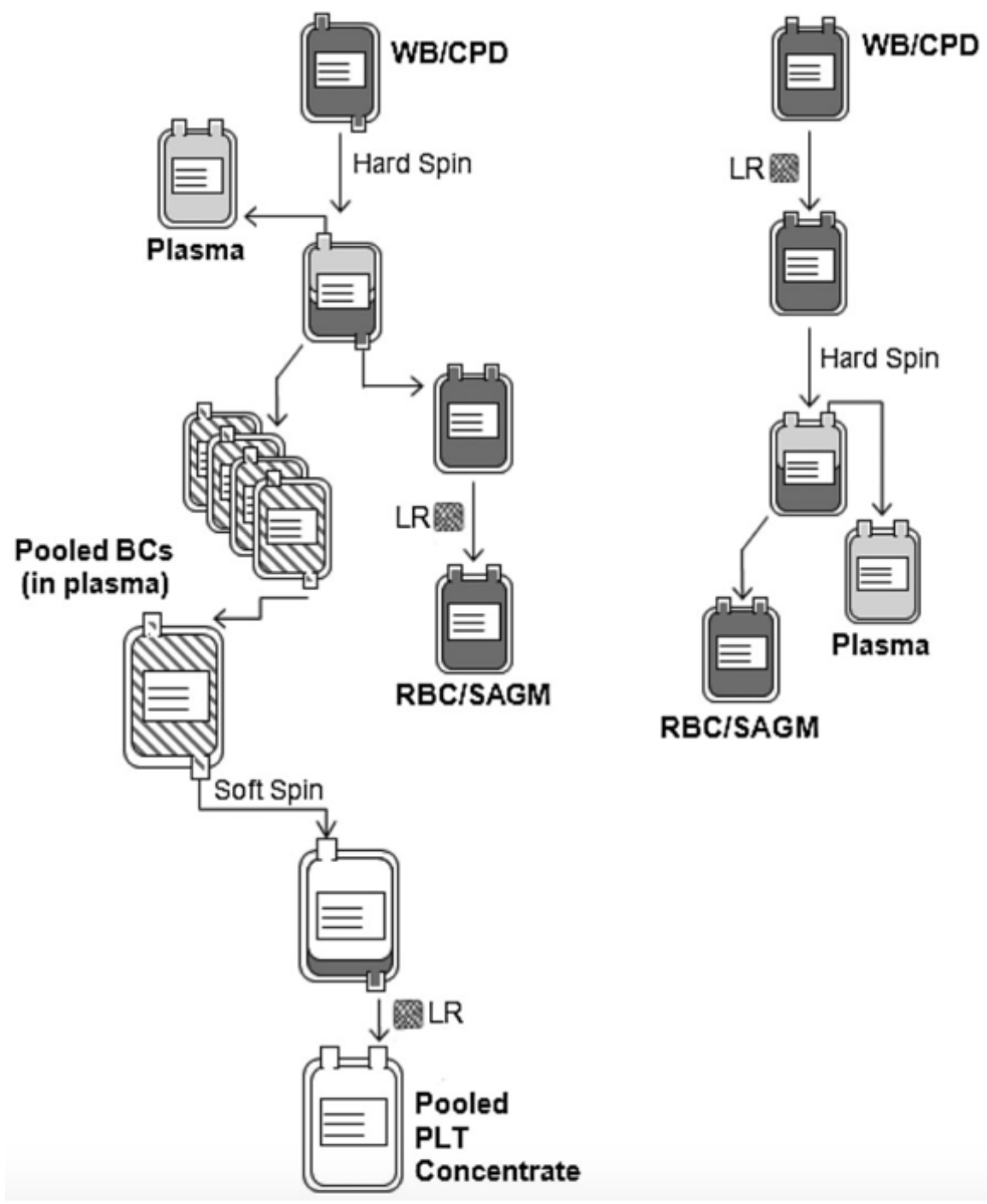

Figure 1-6: (left) BC method of PLT, plasma and RBC extraction; (right) WB method of plasma and RBC extraction. Adapted from [31].

leukoreduced [LR]) via a filtration system [28,29]. In the WB method, donated blood first undergoes LR filtration before being centrifuged to separate the RBCs and plasma; RBCs are subsequently transferred into a PVC bag that contains SAGM [30]. Although both methods differ only in the sequence of centrifugation and filtration, this difference results in the generation of platelets (PLTs) solely through the BC method [31].

The final RBC-SAGM mixture, termed as red cell concentrate (RCC), is sealed from the external environment. The bags are labelled in accordance with CSTM standards, with information such as blood type, production/expiration dates, and various tracking bar codes. The sealed and 
labelled RCCs are stored in an environment of 1-6 degrees Celsius, and are shelved for a maximum of six weeks in the blood banks or, eventually, hospital storage facilities [30,31].

The next two sections of this introduction will describe the storage guidelines developed for optimized RBC storage shelf life, as well as the deleterious effects incurred by RBCs during storage.

\subsection{RBC Biopreservation}

The separation of RBCs from other blood components, suspension in CPD/SAGM additive solutions and storage in plastic bags under specified conditions and timelines (Section 1.3.2), are practices based upon several decades of global advancements in RBC biopreservation research. The fundamental aim of RBC biopreservation is to extend RBC shelf life while maintaining its in vivo viability, and to do so in a convenient medium for efficient reinfusion. Concomitantly, quantitative guidelines have been instated to assess if a given methodology can enhance RBC biopreservation.

\subsubsection{In vitro Hemolysis and In vivo Survivability}

Hemolysis, a term for $\mathrm{RBC}$ rupture, is a negative outcome for $\mathrm{RBC}$ biopreservation as it indicates a depletion in the number of viable RBCs. Furthermore, abnormal amounts of supernatant hemoglobin can have adverse effects on the cardiovascular system upon reinfusion [32]. In one of the first published studies on the biopreservation of RBCs [33], Rous \& Turner outlined a centrifugal method to measure the amount of hemolysis in a sample of blood. Nearly a century later, hemolysis is still a key quantitative measure to assess the quality of RBCs in storage; CBS currently calculates the percentage hemolysis of a sample via the commonly used Drabkin procedure [44].

While hemolysis measurements provide insight into the in vitro quality of an RBC sample, they do not assess the quality of the remaining, intact RBCs and their in vivo functional potential. Ashby in 1919 described a method of monitoring the in vivo lifespan of transfused RBCs [35]. RBCs of two separate blood groups were differentiated through selective agglutination (cell clumping) when mixed with plasma of a specific blood type. This property allowed Ashby to 
monitor the post-transfusion RBC survival by observing the agglutinated (or non-agglutinated) populations of extracted aliquots over time [35].

The differential agglutination technique was frequently used for decades, however, high transfusion volumes and exclusion of autologous transfusions made this technique unsafe and cumbersome [36]. World War II stimulated a revamp of RBC biopreservation research, which by the 1940s brought about advanced, automated techniques in RBC radioactive labelling. Initially, $\mathrm{Fe}^{55} / \mathrm{Fe}^{59}$ isotopes were ideal candidates for tagging $\mathrm{RBCs}$, however, it was found that the required in vivo concentrations were unfavorably large. The $\mathrm{Cr}^{51}$ isotope has since been considered a more suitable tagging isotope, due to its detectability and efficient hemoglobin binding capacity [36,37]. In 1947, Ross et al. [38] devised an arbitrary minimum requirement of $70 \%$ for transfused RBC survival, 24 hours post transfusion, to quantify if a particular media or technique was effective in RBC biopreservation.

In 1985, the U.S. Federal Drug Administration (FDA) officially published minimum criteria for biopreservation techniques to satisfy in order to gain approval for commercial usage [39]. A novel biopreservation technique was required to run trials on RCC blood bags donated by at least 20 volunteers. During the last day of the proposed storage lifetime, RBC samples of each bag were required to:

1) Record an in vitro hemolysis of less than $1 \%$, and

2) Show a mean recovery of $75 \%$ for radiolabeled RBCs at 24 hours post transfusion.

The in vitro and in vivo (respectively) thresholds are still implemented today, albeit with slight modifications in the statistical limitations [40].

\subsubsection{Advancements in RBC Storage}

Hemolysis and in vivo recovery results (Section 1.4.1) are benchmarks to assess if a particular storage technique was effective in biopreservation. Ashby determined from hemolysis measurements that an isotonic concentration of dextrose and sodium citrate was an optimal preservation medium for in vitro RBC storage [35]. In the following decades, modifications in 
the sugar-anticoagulant ratios and addition of buffer components lead to Acid-Citrate-Dextrose (ACD) and CPD additive solutions. Once in vivo survivability criteria became a norm in RBC biopreservation studies, CPD was found to extend the shelf life of blood to 3 weeks, making it more effective than ACD solutions [41,42]. Studies on the influence of storage temperature on post-transfusion in vivo RBC survival showed the benefit of constant refrigeration of stored blood [38].

Advancements in plastics technology in the 1960's caused the replacement of glass bottle storage with plastic bags; compared to bottles, plastics provided flexibility, greater sterility and handling convenience [43]. Studies found that bags comprised of PVC had desirable properties, such as a fourfold reduction in hemolysis compared with glass storage [44]. Multiple bags were soon being linked via plastic tubing for the purpose of developing sterile blood component separation techniques of the CPD-WB solution. Blood component separation was favored primarily due to an overall reduction of component waste, as most medical transfusions do not require infusion of more than one blood component.

By the end of the 1970's, it became apparent that the developed techniques on the separation of RBCs were not practically convenient for transfusion medicine [45]. In the process of separation, a significant volume of CPD nutrients geared for RBC biopreservation were left behind in

plasma. This resulted in a highly viscous RCC that was difficult to transfuse, even when combined with a simultaneous saline infusion [44]. By the early 1980's, RBCs were being diluted and suspended in SAGM, creating an RCC with a suitable hematocrit (RBC percent volume) for direct transfusion. Studies showed that RBCs in SAGM satisfied the FDA guidelines for up to 6 weeks [46]. RBC separation techniques soon included leukoreduction, as a growing body of evidence showed that this procedure, though more expensive [47], would lower hemolysis and increase in vivo survivability significantly $[48,49]$.

\subsection{RBC Storage Lesions}

In vitro $\mathrm{RBC}$ hemolysis and post-transfusion in vivo survivability are established methods for the quality assessment of stored RBCs (Section 1.4). Hemolysis monitors the percentage of ruptured RBCs in vitro, however, it cannot provide information on the functional capacity of intact RBCs. 
In vivo survivability measurements determine the percentage of non-viable RBCs as a function of time, but this measure cannot infer the functional integrity of the circulating transfused RBCs. In order to determine the functional potential of stored RBCs upon reinfusion, a thorough understanding of the biochemical state of RBCs within their storage environment over the storage period is required.

\subsubsection{RBC Storage Lesion: Definition}

Seminal work published in the 1950's investigated RBC cell changes taking place in in vitro storage conditions. An increase in osmotic fragility (susceptibility for hemolysis) and a decrease in glucose utilization were some of the major changes found in RBCs stored in vitro in ACD [50,51], but not in aging RBCs in vivo [52]. Studies were expanded to monitor stored RBCs of different species, with similar trends observed; investigators concluded that RBC changes during in vitro storage are due to factors independent of the physiological senescence of the cell [52].

Gabrio et al. coined the term "RBC storage lesion" to describe a composition of biochemical and biomechanical alterations induced by in vitro storage conditions, which lead to deleterious effects of the cell function and viability [52]. In the same decade, Gibson and colleagues found a correlation between storage lesion severity and an increase in hemolysis, as well as an inverse correlation with in vivo RBC survivability [53]; These findings provided evidence that storage lesions could affect the quality of transfused RBCs.

The characteristics of an RBC storage lesion are initiated by metabolic changes that occur in the stored RBC [54,55]. ATP, a critical metabolite for cell function, has been found to decrease in concentration during storage [56]. Compared to room temperature, the rate of RBC glycolysis has been found to reduce tenfold in a hypothermic storage environment [57]. Lactate and protons produced by the glycolytic pathway create an increasingly acidotic environment, since there is no mechanism to neutralize the $\mathrm{pH}$ shift in vitro [57-59]. Enzymes such as kinases used in the glycolytic pathway reduce in efficacy due to lowered $\mathrm{pH}$, causing a decrease in ATP production [60].

The features of an RBC storage lesion include some effects that can be reversed when the RBCs 
are transfused in vivo. In an attempt to compensate for the slowing rate of glycolysis, phosphatases are activated and break down 2,3-DPG into components that are used in the glycolytic pathway to produce more ATP [19]. Low storage temperatures continue to suppress glycolysis rates, leading to a sharp decline of 2,3-DPG concentrations within the first week of storage (Figure 1-9). The lack of 2,3-DPG results in a higher affinity of hemoglobin for $\mathrm{O}_{2}$ (Section 1.2.4). It can take between 1-3 days to restore intracellular levels of 2,3-DPG post transfusion [61,62]. Cation pumps - amongst other membrane-based proteins - lose functionality at low storage temperatures, resulting in a large influx and efflux of sodium and potassium respectively [63]. Reinfused RBCs can take up to one day to restore the sodium gradient and up to 4 days to restore the potassium gradient [59].

While some RBC storage lesion changes are reversible when reinfused in vivo, various irreversible events occur as ATP metabolism slows down [64,65]. As ATP availability diminishes, reductases begin to lose capacity to maintain the oxidative presence within the cell [66]. One source of this increased oxidative stress originates from radicals such as $\mathrm{O}_{2}^{-}$(Equation 1-2), that are not reduced as frequently when glycolysis slows down [58]. The prolonged existence of this superoxide radical increases the probability of forming hydroxyl radicals via the Fenton reaction [67]. Hydroxyl radicals generate a cascade of radical species that target and damage membrane phospholipids and associated cell proteins that maintain cell functionality and structure [68-71]. RBCs begin the removal of the damaged membrane via vesiculation, shipping toxic byproducts out of the cell and into the supernatant in microvesicles [72-75]. The irreversible loss of membrane results in RBC shape changes, where regular biconcave discs morph into rigid spheres containing bumps of budding microvesicles [76,77]. Figure 1-7 provides a summary of the major components of the RBC storage lesion [78]. Figure 1-8 displays changes in select RBC storage lesion features as a function of storage duration [56]. 


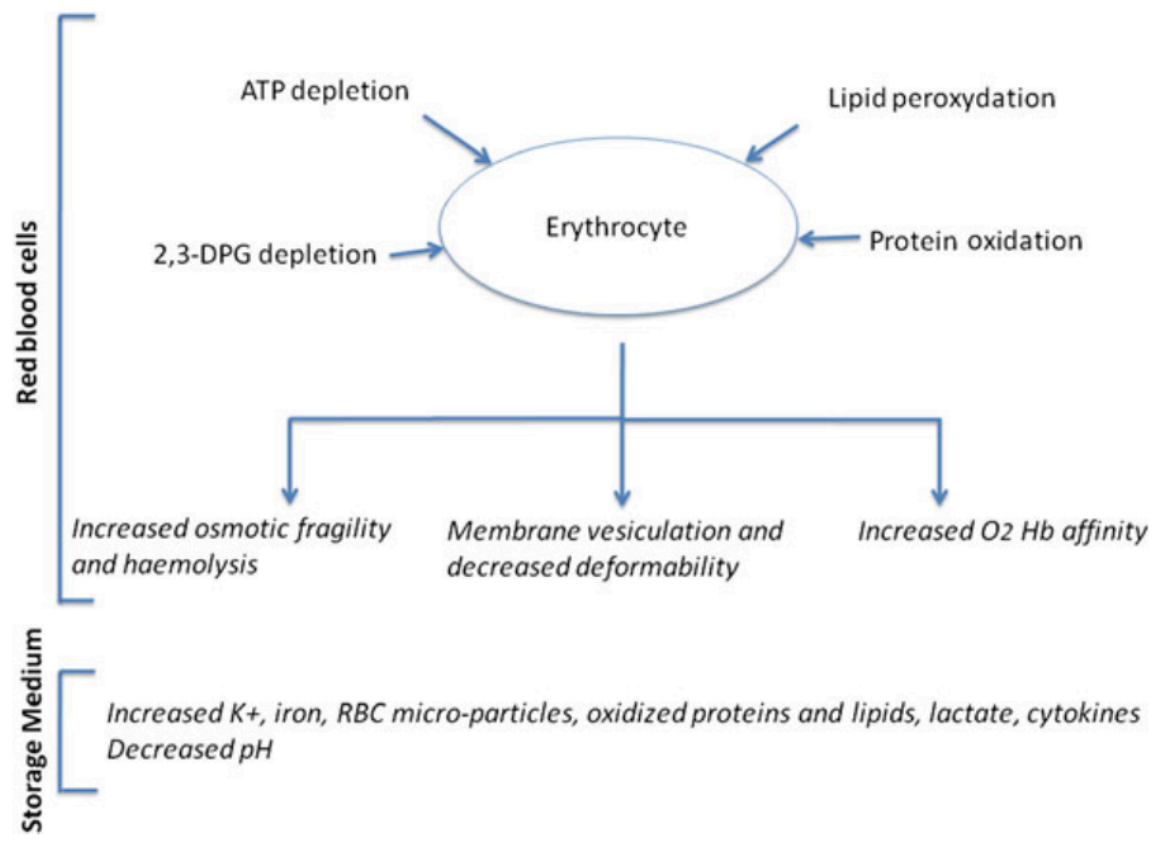

Figure 1-7: Major components of the RBC storage lesion, consequences to the cell and storage medium. Adapted from [78].
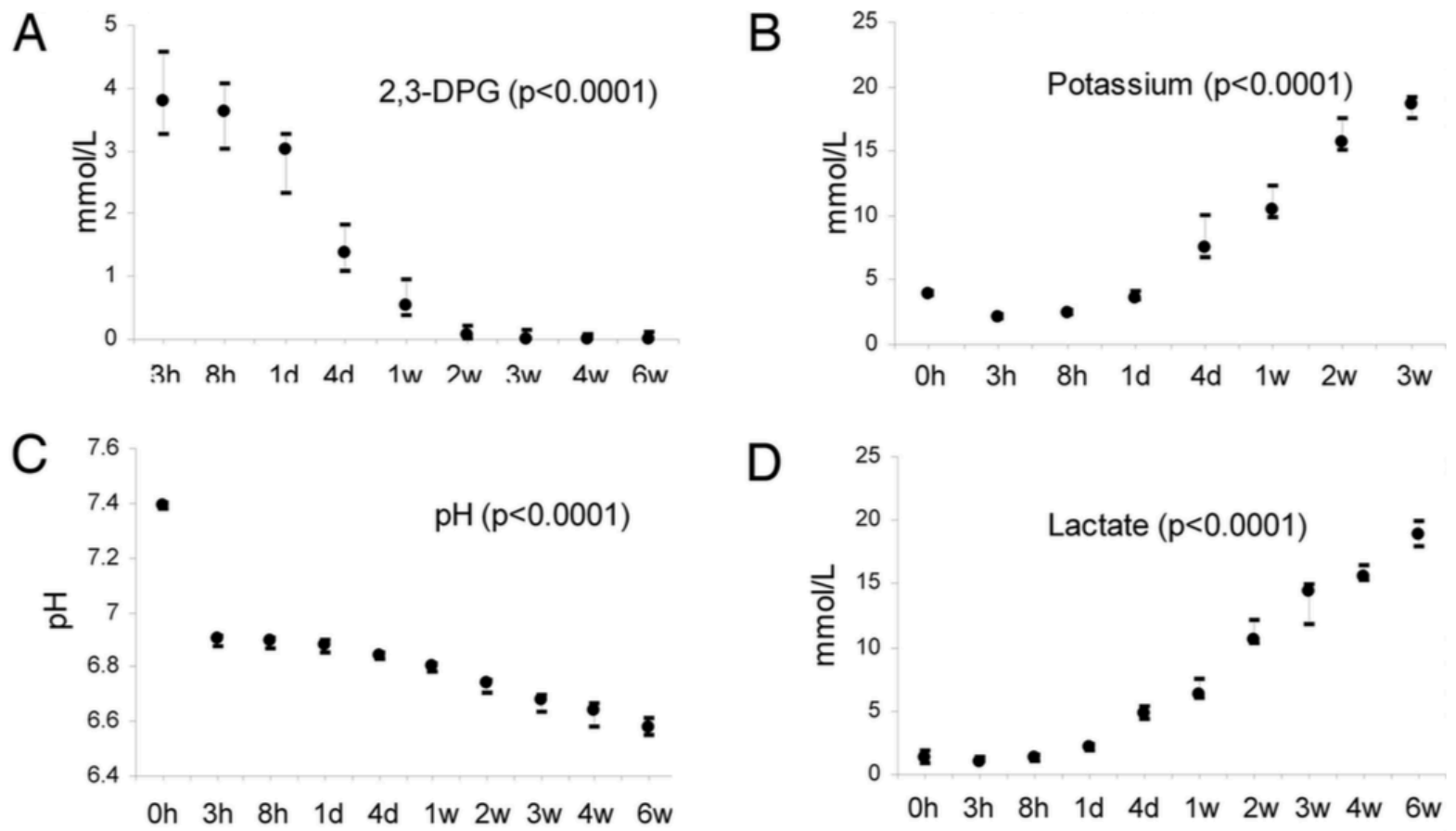

Figure 1-8: Intracellular 2,3-DPG concentration (A), as well as changes in supernatant potassium (B), $\mathrm{pH}(\mathrm{C})$ and lactate levels (D) as a function of storage duration. Data points are median with 25 and 75 percentile ranges. $P$ values represent significance of change over time. Adapted from [56]. 


\subsubsection{RBC Storage Lesion: Change in $\mathrm{SO}_{2}$}

As illustrated in Figure 1-9, there is a rapid consumption of 2,3-DPG in stored blood within the first couple of weeks, primarily due to phosphatases breaking down the molecule for use in the glycolytic pathway (Section 1.5.1). The glycolytic pathway orients towards this breakdown of 2,3-DPG in a hypothermic, acidotic environment, as is the case for the in vitro storage conditions of RCCs [79,80]. As 2,3-DPG concentrations plummet within the first two weeks of storage, an unregulated increase occurs in the affinity of hemoglobin to $\mathrm{O}_{2}$ [81]. In terms of the ODC, a 2,3DPG concentration decrease causes a right-shift that produces a higher $\mathrm{SO}_{2}$ value for any given

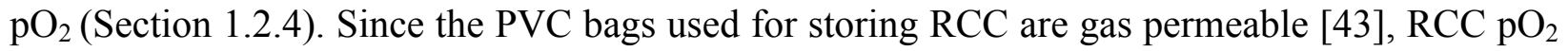
is in equilibrium with the surrounding atmospheric $\mathrm{pO}_{2}$. Consequently, $\mathrm{RBC}$ continually bind to more $\mathrm{O}_{2}$ during storage; this trend is captured in Figure 1-9, where Bennett-Guerrero et al. measured $\mathrm{RBC} \mathrm{SO}_{2}$ over a six-week storage duration [56].

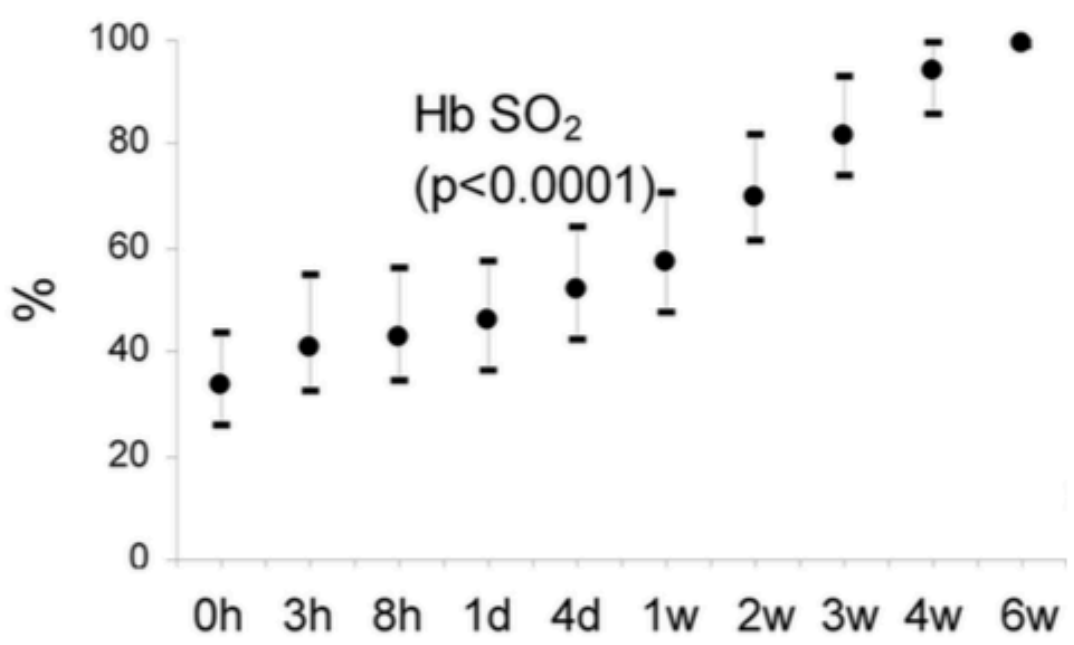

Figure 1-9: $\mathrm{RBC} \mathrm{SO}_{2}$ percentage changes as a function of storage duration. Data points are median with 25 and 75 percentile ranges. $P$ values represent significance of change over time. Adapted from [56].

\subsubsection{RBC Storage Lesion: Change in Morphology}

Over storage lifetime, the RBC shape changes from a biconcave disc to a sphere with spiky projections (or spicules), which is a snapshot of vesicles in the process of pinching off the 
membrane and exporting toxic waste out of the cell (Section 1.5.1). The expulsion of membrane phospholipids and proteins responsible for cell structure results in a gradual loss of biconcavity, as well as a reduction in cell diameter [82]. Hence, by observing images of stored RBC, the severity of a storage lesion could be inferred from characteristics such as the number of spicules, cell shape and diameter.

RBC morphology characterization was pioneered by Furchgott [83] with modifications made by Gardos and colleagues [84]. The current convention implemented by CBS characterizes RBC storage lesion morphology into 6 categories [85]. The devised terminologies and categories, whose features are outlined in Table 1-2, represent a sequence of distinct morphological changes that correlate with the increasing structural degradation of RBCs.

A smooth disc, also known as a discocyte, represents the regular biconcave RBC with a round edge and clear central pallor (Section 1.1.2). A crenated disc identifies the initial point of the membrane budding process, and differs from a smooth disc by the presence of lumpy edges $[86,87]$. A crenated discoid illustrates the initial degradation of the central pallor as well as the beginning of vesiculation [86,87]. Both the crenated disc and crenated discoid are collectively termed as an echinocyte, and are able to revert back to the discocyte morphology if exposed to a nutrient-rich isotonic environment [82]. These three morphologies are termed "reversible", implying that they can revert to a regular RBC morphology upon reinfusion [88].

As membrane proteins and cytosol content start exiting the cell, there is a loss of biconcavity and a shift toward a spherical shape, which is represented by the term crenated spheroid $[86,87]$. The crenated sphere identifies a complete morphological shift into a sphere, with the vesiculation process nearing its end. The smooth sphere, also termed as a spherocyte, illustrates the end of cell membrane budding and a complete loss of structural viability of an RBC [86,87]. These three morphologies are termed "irreversible" and are labelled as spheroechinocytes $[88,89]$.

A key property of the RBC storage lesion, with respect to morphology, is that the quantity of irreversible spheroechinocytes increase as a function of storage duration. Figure 1-10 showcases scanning electron microscopy (SEM) images taken by Berezena and colleagues [90], portraying 


\begin{tabular}{|c|c|c|c|}
\hline TERMINOLOGY & $\begin{array}{c}\text { REVERSIBILITY \& } \\
\text { OTHER } \\
\text { TERMINOLGY } \\
\end{array}$ & $\begin{array}{l}\text { DISTINGUISHING } \\
\text { FEATURES }\end{array}$ & IMAGE \\
\hline Smooth Disc & \multirow{3}{*}{$\begin{array}{l}\text { REVERSIBLE } \\
\text { (Discocytes) } \\
\text { (Echinocytes) }\end{array}$} & $\begin{array}{c}\text { Smooth, Circular Edge } \\
\text { Clear Central Pallor } \\
\text { Regular Diameter }(\sim 6-8 \mu \mathrm{m})\end{array}$ & \\
\hline Crenated Disc & & $\begin{array}{l}\text { Slightly Lumpy Edge, Indicating } \\
\text { Spicule Formation } \\
\text { Clear Central Pallor } \\
\text { Regular Diameter } \\
\end{array}$ & \\
\hline Crenated Discoid & & $\begin{array}{c}\text { Irregular Edge, Numerous } \\
\text { Round Spicules } \\
\text { Faint \& Irregular Central Pallor } \\
\text { Regular Diameter }\end{array}$ & \\
\hline Crenated Spheroid & \multirow{3}{*}{$\begin{array}{l}\text { IRREVERSIBLE } \\
\text { (Spheroechinocytes) } \\
\text { (Spherocytes) }\end{array}$} & $\begin{array}{l}\text { Irregular Edge, Numerous } \\
\text { Pointy Spicules } \\
\text { Very Faint \& Small Central } \\
\text { Pallor } \\
\text { Slightly Smaller Diameter }\end{array}$ & \\
\hline Crenated Sphere & & $\begin{array}{l}\text { Numerous Hair-Like Projections } \\
\text { Spherical, Dense Interior } \\
\text { Smaller Diameter }\end{array}$ & \\
\hline Smooth Sphere & & $\begin{array}{l}\text { Few Extremely Faint Spicules } \\
\text { Spherical, Dense Interior } \\
\text { Smaller Diameter }(\sim 4-5 \mu \mathrm{m})\end{array}$ & \\
\hline
\end{tabular}

Table 1-2: Categories of RBC storage lesion morphology and their features [83-85] and reversibility [82,86-88], and an example SEM image of each category (adapted from [88]). 

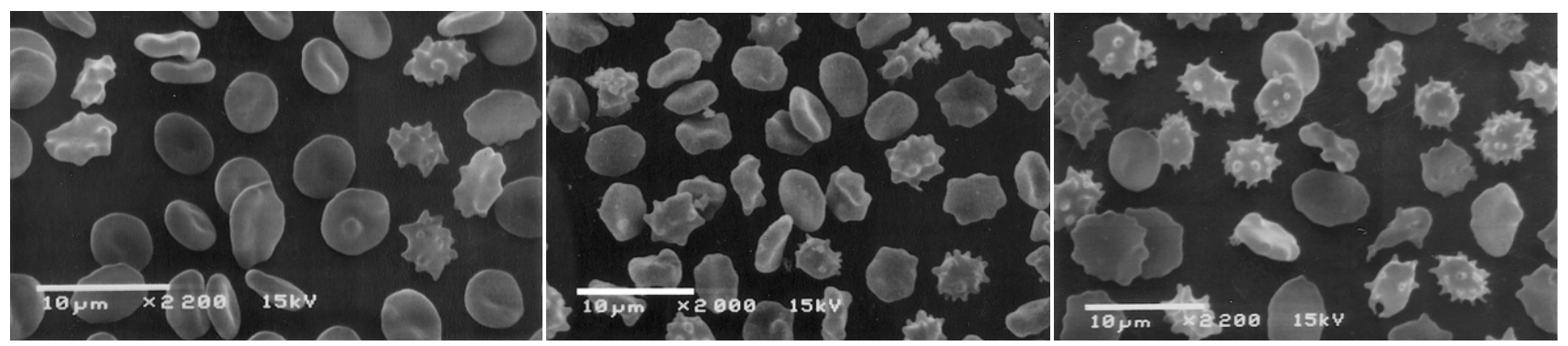

Figure 1-10: Stored RBC morphology distribution as a function of storage duration; (left) Day 5 of storage is dominated by discocytes; (center) Day 14 of storage is dominated by echinocytes and (right) Day 42 is dominated by irreversible spheroechinocytes. Adapted from [90].

the change in morphology distribution favoring a continuously increasing population of irreversibly damaged RBCs over the six-week storage duration.

\subsubsection{RBC Storage Lesion: Effect on RBCs in Vivo}

$\mathrm{RBC}$ biopreservation research and development aims to prolong the in vitro storage of RBCs without violating established guidelines on percent hemolysis and in vivo cell recovery (Section 1.4). Quantitatively, hemolysis and cell recovery provide the percentage of RBCs that have survived through storage and transfusion; what these parameters do not assess is the functional capacities of those RBCs. It is plausible for a biopreservation technique to yield low hemolysis and high cell survivability post transfusion, but have a large percentage of the survived RBC population affected by storage lesions. Hence, it is important to understand how the characteristics of RBC storage lesions affect the functionality of cells in vivo.

One of the major features of the storage lesion is the gradual degradation of the RBC cell membrane. Maintenance of RBC function is compromised when characteristics such as electrolyte gradients, protein pumps, membrane receptors and more are negatively affected by oxidative damage to the cell membrane [91]. Membrane loss due to vesiculation leads to the irreversible spheroechinocyte morphology (Section 1.5.3) [92]. Since deformability is essential for RBCs to traverse through the microvasculature (1.1.2), an increase in rigid spheroechinocyte population reduces the ability of RBCs to perfuse efficiently, potentially causing local hypoxia $[90,93]$. 
Another key feature of the storage lesion - leading to reduced functionality - is the depletion of 2,3-DPG and consequent increase in oxygen affinity (Section 1.5.2). Until intracellular 2,3-DPG values are restored after many hours [61,62], abnormally sharp gradients of $\mathrm{pO}_{2}$ are needed in order to induce the release of $\mathrm{O}_{2}$ from hemoglobin. Hence, RBCs lacking 2,3-DPG are unable to efficiently carry out their primary task of releasing $\mathrm{O}_{2}$ to surrounding tissues [81].

\subsubsection{RBC Storage Lesions: Clinical Outcomes}

There is evidence linking RBC storage lesions (and how they cause a decline in the in vitro quality of RBCs (Sections 1.5.1-1.5.3) [56-59,81]) with impaired in vivo oxygen augmentation capacity (Section 1.5.4) [90-93]. Furthermore, the data from these studies strongly suggest that the functional capacity of RBCs decline in proportion to storage duration. The six-week storage duration for RCCs in SAGM was set by guidelines on hemolysis and in vivo cell survivability (Section 1.4.1), and not on the subsequent evidence regarding the existence of RBC storage lesions and their increasingly adverse effects over time. Hence, over recent decades, there have been a number of investigations on the clinical consequences associated with reinfusing RBC storage lesions into recipients, and if so, whether the adverse effects observed are dependent on storage duration.

In the early 1990's, correlations between the age of transfused RBCs and increased adverse outcomes were being reported, specifically for recipients under a vulnerable state of health [94]. For example, Marik \& Sibbald found evidence of splanchnic ischemia occurring in critically ill patients that received transfusions of older RBCs [95]. In a seminal paper by Purdy and colleagues, an association was found between RCC storage duration and risk of mortality in septic patients in the intensive care unit (ICU) [96]. These results challenged the integrity of the six-week storage duration implemented by most global RBC storage centers, and encouraged more detailed investigations into the association between $\mathrm{RBC}$ age and other morbidities.

Subsequent studies have tracked morbidities that included length of stay, multiple organ failure, thrombosis, renal failure and other post-surgery parameters; populations were expanded to those in trauma, undergoing cardiac surgery and admissions into other hospital environments. Review 
articles have summarized some of the accumulated clinical data [78,97-99]. Aubron and colleagues found that among 32 studies of various morbidities, patient populations and cofounders, 56\% reported deleterious effects due to RCC storage, while the rest were inconclusive [78]. Lelubre \& Vincent found that $50 \%$ of 55 studies produced a significant correlation between RBC age and morbidities [97]. Wang et al. and Remy et al. determined (respectively) that $81 \%$ of 21 studies and $67 \%$ of 31 studies focused on mortality had a correlation between RBC age and risk of mortality [98,99].

The reviews discussed do not reach the same conclusions on the link between RBC age and clinical impact. Much of this discrepancy can be attributed to the variation in study designs, statistical methods, sample populations and morbidities analyzed. For example, Remy et al. presented the varying definitions on what was considered "fresh" and "old" RBCs in 20 independent studies (Figure 1-11) [99].

Median storage age for individual observational studies

$\square$ Mean storage age for individual observational studies

O Median storage age for individual randomized controlled trials

$\square$ Mean storage age for individual randomized controlled trials

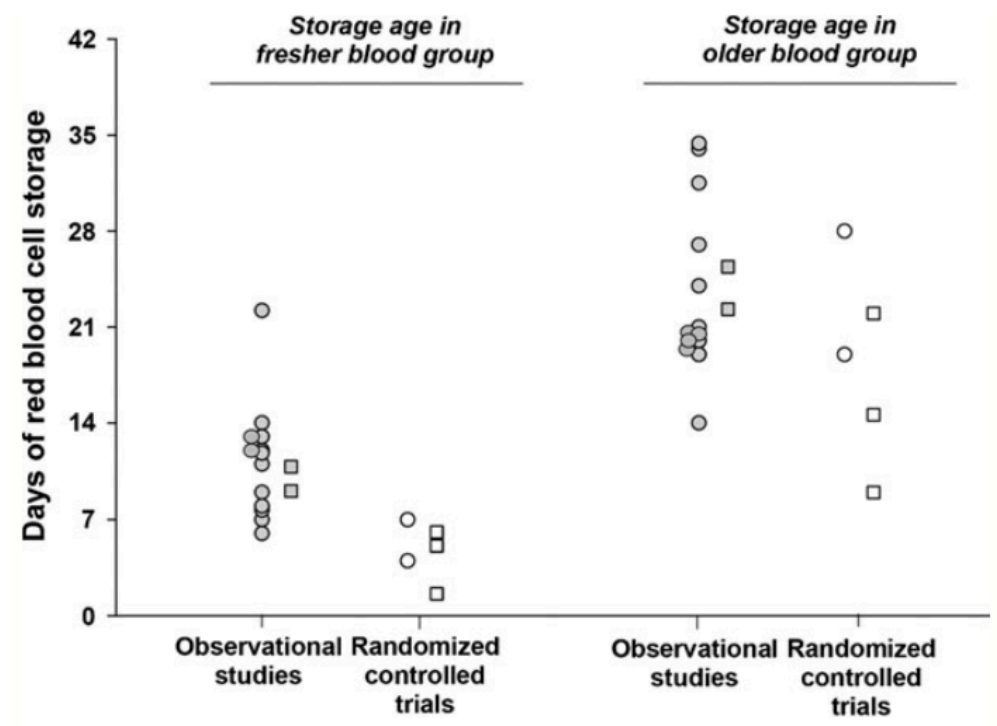

Figure 1-11: Variation in the mean/median of what was considered "fresh" and "old" RCC according to 15 observational studies and 5 RCTs. Adapted from [99]. 
Recognition of the issues behind observational studies has brought on increasing demand for random clinical trials (RCTs), a gold standard in evidence based medical research [100,101]. In the context of storage lesion severity as it relates to mortality, conclusions from RCTs have found no dependence on the storage age of transfused RBCs, unlike those suggested by many observational studies [98,99]. Figure 1-12 displays the discrepancy between 31 observational studies and 6 RCTs, all completed between 2001 and 2015 [99]. The RCTs reviewed by Wang et al. and Remy et al. were small in quantity and contained small sample populations, and hence it is plausible that these factors contributed to the results $[98,99]$.

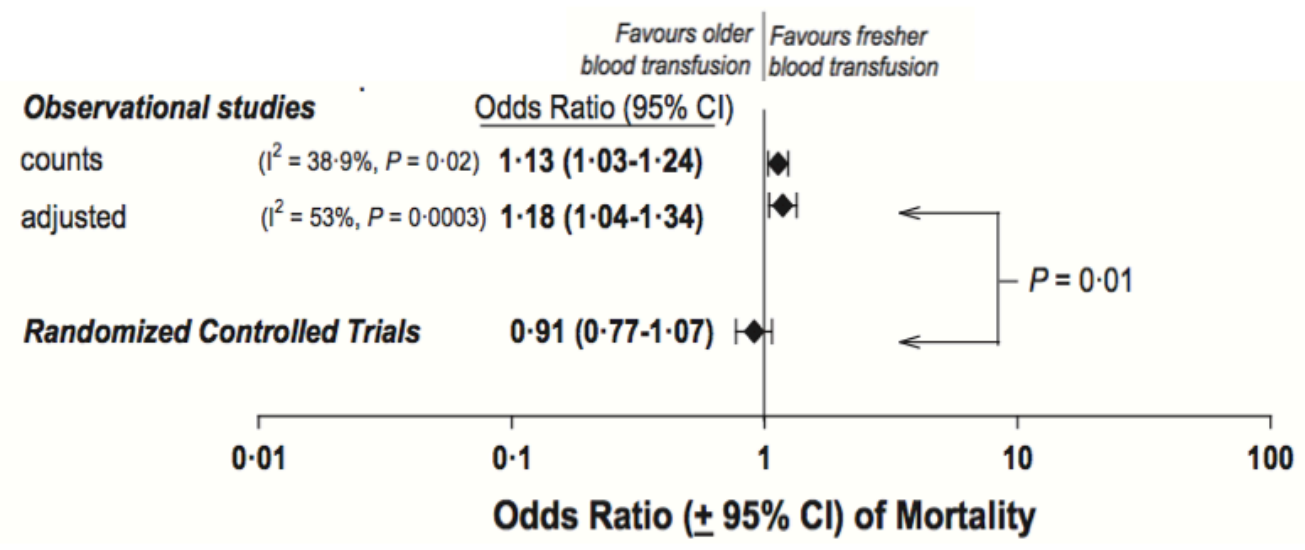

Figure 1-12: Discrepancy in odds ratios between 31 observational studies and 6 RCTs, in determining if fresher or older (regular) RCCs lower mortality rates. Observational studies show an increase in odds of mortality (1.13) when transfusing older blood, while RCTs show no advantage for the transfusion of fresh blood. $\mathrm{I}^{2}$ refers to the statistical measure of heterogeneity. Adjusted refers to confounder adjustments, where applied. Adapted from [99].

RCTs, though superior to observational studies in study design and confounder minimization, are more cost and labor intensive. Hence their conclusions have been limited by small, singlecentered sample populations [100]. In recent years however, several multi-centered, large population RCTs have been carried out through global initiatives and collaborations. The conclusions behind some recently published studies have supported previous RCT findings. For example, Steiner and colleagues found no advantage for transfusing fresher RCCs into cardiac surgery recipients [102]. Similarly, both Lacroix et al. [103] and Heddle et al. [104] found no improvement in mortality rates due to transfusion of fresh RCCs versus old RCCs. 
Although there seems to be mounting evidence suggesting that RBC storage lesion severity (or age of RCCs) does not affect the rate of mortality, none of the RCTs - and in fact only a few observational studies - have assessed the effect of transfusing RCCs in their last week of storage ( $\sim$ days 35-42). Deleterious effects are most likely to occur during this storage period, and hence an investigation of the clinical outcomes during this time frame would be essential before any stance on the dependence on RCC storage age could be accepted conclusively. Furthermore, the limited number of RCTs indicates that conclusions should treated with caution. Moreover, RCTs have focused on the link between RCC age and mortality, not morbidity [105].

A significant body of research has been published on characterizing RBC storage lesions (Section 1.5.1), the in vitro effect of RCC storage on RBCs (Sections 1.5.2,1.5.3), and the in vivo effects post transfusion (Sections 1.5.4,1.5.5). However, the clinical impact of these findings is not clear. In order to better understand the impact RBC storage lesions may have in vivo, better techniques are needed to monitor the state of RBC lesions prior to transfusion. Currently, there is no RBC storage lesion detection technique or assay used in the hospital environment prior to transfusion. The only parameter available for clinicians and scientists to infer the state of the $\mathrm{RBC}$ storage lesions is storage age. A technique that could measure parameters such as the morphology of $\mathrm{RBC}$ populations or $\mathrm{SO}_{2}$ prior to transfusion would help establish the link between the functional changes of RBC storage lesions and their clinical impact.

The next four sections of this introduction will introduce how optical techniques can be used to obtain quantitative information with respect to the biophysical characteristics of RBC storage lesions. 


\subsection{RBCs and Optical Properties/Techniques}

\subsubsection{Optical Parameters of RBCs at the VIS-NIR Optical Regime}

Optical properties of the blood are determined by measuring the change in light intensity due to absorption and scattering [106]. The optical properties of a particular medium can be represented by the absorption coefficient $\left(\mu_{\mathrm{a}}\right)$, scattering coefficient $\left(\mu_{\mathrm{s}}\right)$ and the anisotropy factor $(\mathrm{g})$ [107]. $\mu_{\mathrm{a}}$ and $\mu_{\mathrm{s}}$ represent the probability, within a unit distance, of an absorption and scattering event to occur, respectively. Anisotropy parameter ranges between -1 to 1 to represent the direction of scattering with respect to incident light, where a value of 1 indicates complete forward scattering and a value of 0 indicates unidirectional (isotropic) scattering [107]. Optical parameters are wavelength dependent, where by varying the wavelengths of incident radiation, different values of the optical properties are measured.

Within the visible and near-infrared (VIS-NIR) portion of the electromagnetic spectrum, hemoglobin is the dominant absorber in blood [108]. In this regime $(\sim 0.3-1.1 \mu \mathrm{m})$, the $\mu_{\mathrm{a}}$ of RBCs are 1-3 order of magnitudes larger than that of plasma and other cellular components [106]. At energies outside the VIS-NIR regime, water and plasma proteins become significant absorbers [109]. Figure 1-13 showcases the $\mu_{\mathrm{a}}$ of oxyHb and deoxyHb in the optical range discussed, as well as the optical absorption behavior for water, plasma proteins and melanin (a light absorbing species in tissue).

A closer look at the red curves in Figure 1-13 reveal distinct spectral signatures that exist in the absorption spectra of oxyHb and deoxyHb. Oxygen binding result in conformational changes in the hemoglobin molecule, which changes the vibrational state of hemoglobin and consequently changes the absorption cross sectional area [110]. Furthermore, different oxidation states of the heme iron introduce new spectral features. Dyshemoglobins like metHb (Section 1.2.2) possess unique spectral features in the VIS-NIR optical regime [110]. 


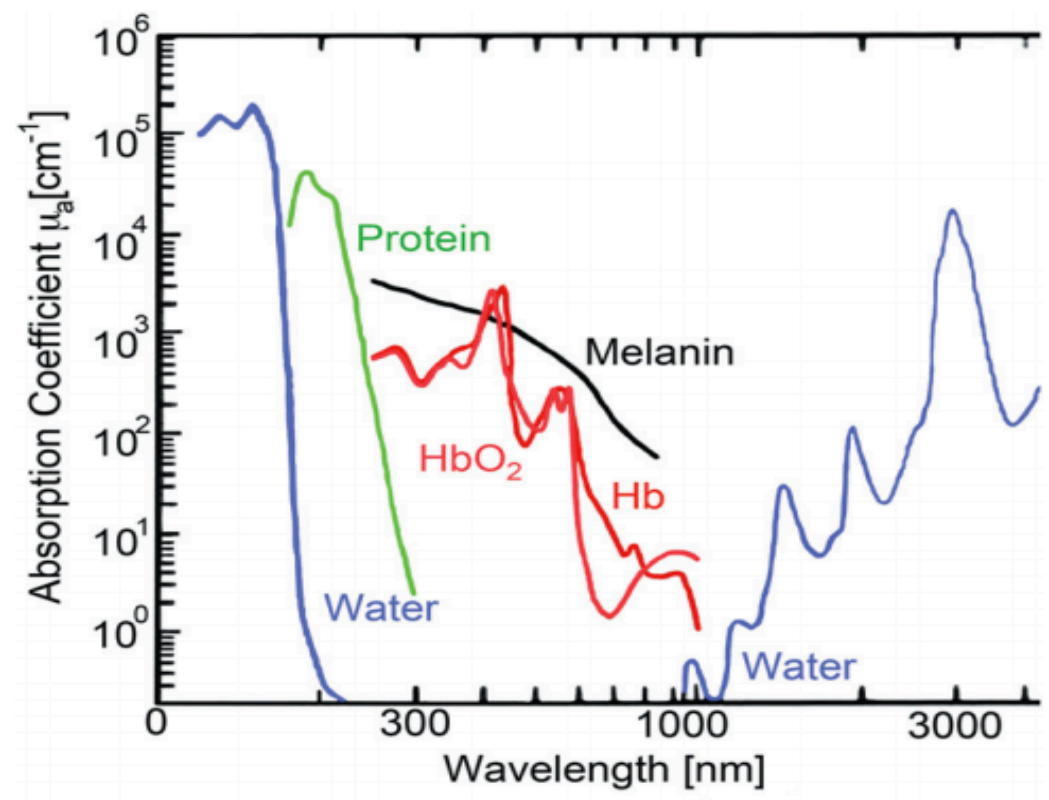

Figure 1-13: Absorption coefficients of the two major functional states of hemoglobin (red), plasma proteins (green) and water (blue). Melanin (black) is a major absorbing species in tissue. Adapted from [111].

\subsubsection{Absorption Spectroscopy and Hemoglobin Concentration}

Within the VIS-NIR wavelength range, RBCs exhibit highly anisotropic, forward scattering ( $\mathrm{g}$ 0.9) with a scattering coefficient that decreases monotonically over the optical regime $[106,108,110]$. Scattering in this regime primarily occurs due to the variation in the index of refraction between the RBC membrane and the surrounding medium [112]. If RBCs are lysed such that only the hemoglobin molecules are suspended in the medium, scattering can be greatly reduced. Under low concentrations, it can be assumed that the contribution of scattering to the attenuation of light can be ignored [112-114]. Hence, under these conditions, the attenuation of light is assumed to be dominated by absorption and can be described accurately by the BeerLambert law [114]. The Beer-Lambert law states that the optical intensity traversing through a medium containing absorbing species is a function of the absorbing medium thickness and concentration.

In absorption spectroscopy, the intensity of light traversed through an absorbing medium is measured in relation to the incident intensity (or transmittance) for a spectrum of wavelengths. The Beer-Lambert law (illustrated in Figure 1-14), whose derivation is provided in Appendices 
B, relates the absorbance (A) (inverse logarithmic transmittance) at a particular wavelength $(\lambda)$ with the product of the tabulated molar extinction coefficient $(\varepsilon)$ at that wavelength, the molar concentration of the absorbing species $(C)$ and the path length of light $(x)$ :

$$
A(\lambda)=\varepsilon(\lambda) \cdot C \cdot x
$$

The concentration of absorber C can be solved by rearranging Equation 1-4. However, if there are multiple (N) absorbing species, Equation 1-4 is modified to:

$$
\mathrm{A}_{\text {tot }}^{\lambda}=x \cdot \sum_{n=1}^{N} \varepsilon_{n}^{\lambda} \cdot \mathrm{C}_{n}
$$

A unique solution for Equation 1-5 cannot be obtained unless at least $N$ equations are produced. Absorption spectroscopy involves the illumination of the absorbing species by at least $\mathrm{N}$ wavelengths and measuring the resulting fluctuations in transmitted light intensity. Assuming that a sample of RBCs contains various concentrations of oxyHb, deoxyHb, metHb and other dyshemoglobins (Section 1.2.2), Equation 1-5 can be applied for at least four wavelengths to solve for the concentration of each absorbing species. Molar extinction coefficients for different states of hemoglobin are available in tabulated format [116,117]. The application of this technique to blood gas analysis is discussed in further detail in Section 2.2.2.

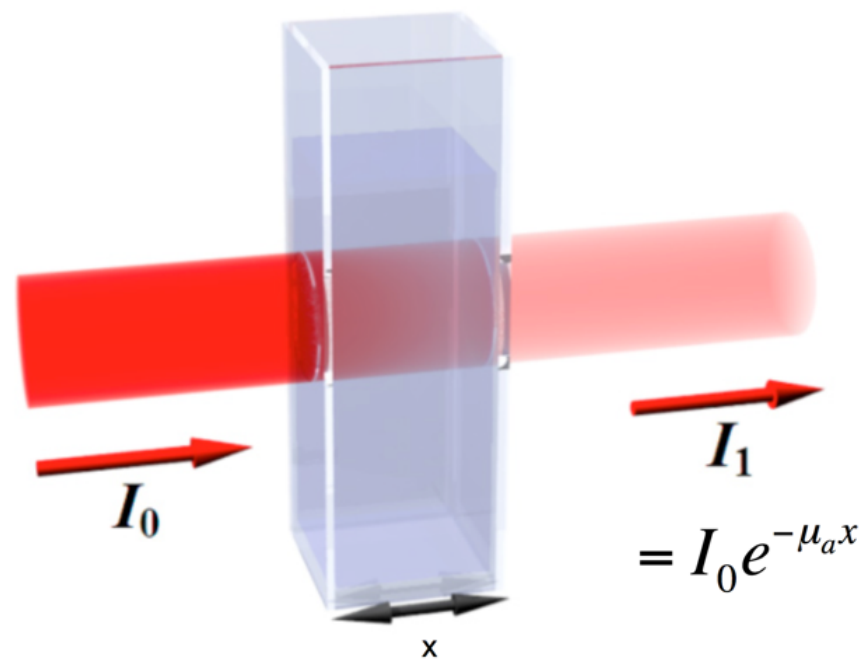

Figure 1-14: Attenuation of a VIS-NIR beam $\mathrm{I}_{0}$ can be attributed to mostly $\mathrm{RBC}$ absorption, under certain circumstances. Derivation of expression of $\mathrm{I}_{1}$ is found in the appendices (C). Adapted from [115]. 


\subsubsection{Oximetry Technique and $\mathrm{SO}_{2}$ Determination}

In most cases, it can be assumed that the dominant hemoglobin states are oxyHb and deoxyHb (Section 1.2.2). A minimum of two equations must be prescribed to solve for the unknown concentrations of oxyHb and deoxyHb:

$$
\begin{aligned}
& A_{\text {tot }}^{\lambda_{1}}=\left(\varepsilon_{\mathrm{HbO}_{2}}^{\lambda_{1}} C_{\mathrm{HbO}_{2}}+\varepsilon_{\mathrm{Hb}}^{\lambda_{1}} C_{\mathrm{Hb}}\right) \cdot x \\
& \mathrm{~A}_{\text {tot }}^{\lambda_{2}}=\left(\varepsilon_{\mathrm{HbO}_{2}}^{\lambda_{2}} \mathrm{C}_{\mathrm{HbO}_{2}}+\varepsilon_{\mathrm{Hb}}^{\lambda_{2}} \mathrm{C}_{\mathrm{Hb}}\right) \cdot x
\end{aligned}
$$

Manipulations of these equations, along with the definition of $\mathrm{SO}_{2}$ (Equation 1-3) leads to the following expression of $\mathrm{SO}_{2}$, whose derivation is provided in the Appendices $(\mathrm{C})$ :

$$
\mathrm{SO}_{2}=\frac{\mathrm{A}_{\text {tot }}^{\lambda_{2}} \cdot \varepsilon_{\mathrm{Hb}}^{\lambda_{1}}-\mathrm{A}_{\text {tot }}^{\lambda_{1}} \cdot \varepsilon_{\mathrm{Hb}}^{\lambda_{2}}}{\mathrm{~A}_{\text {tot }}^{\lambda_{1}} \cdot \Delta \varepsilon^{\lambda_{2}}-\mathrm{A}_{\text {tot }}^{\lambda_{2}} \cdot \Delta \varepsilon^{\lambda_{1}}}
$$

where the symbol $\Delta \varepsilon^{\lambda}$ is a shorthand notation for the constant:

$$
\Delta \varepsilon^{\lambda}=\varepsilon_{\mathrm{HbO}_{2}}^{\lambda}-\varepsilon_{\mathrm{Hb}}^{\lambda}
$$

Two wavelengths are ideally chosen at either ends of an isosbestic point, a term used to describe the intersection of each absorber's molar extinction coefficient spectra. Figure 1-15 shows the molar extinction spectra of oxyHb and deoxyHb, displaying various isosbestic points in the VISNIR optical regime. However, wavelengths are normally chosen around the $800 \mathrm{~nm}$ isosbestic point, since the absorption coefficients of hemoglobin are low in this region, allowing for further in vivo penetration depths. As Figure 1-15 depicts, deoxyHb is the dominant absorber for wavelengths less than $800 \mathrm{~nm}$, while oxyHb is dominant above $800 \mathrm{~nm}$. The benefit to this ratiobased approach to $\mathrm{RBC} \mathrm{SO}_{2}$ calculation is that it need not be constrained by the limitations attached to absorption spectroscopy. 


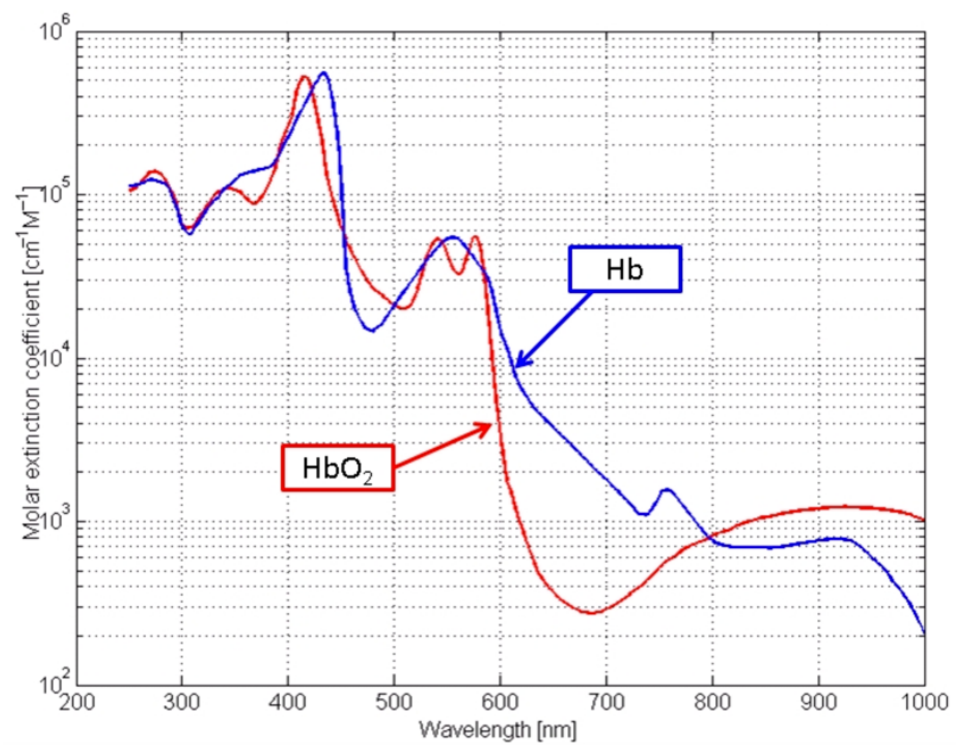

Figure 1-15: Plot of the molar extinction coefficients of oxyHb (indicated by $\mathrm{HbO}_{2}$ ) and deoxyHb (indicated by $\mathrm{Hb}$ ) across the VIS-NIR optical window. Adapted from [117].

\subsection{RBCs and Optical Technologies}

\subsubsection{Transmitted Light Microscopy and RBC Morphology Assay}

Transmitted light microscopy (also known as bright field microscopy) relies on differences in light transmission through a cell to form images [119]. Their primary function, providing images with high resolution, requires bright illumination and large objective lenses to collect light transmitted through the sample [119]. To record images with suitable contrast and resolution, RBC samples are typically fixed, smeared and stained [120].

The CBS morphology assay was adapted from Usry et al.'s method of acquiring SEM images from suspended RBCs [85], and is instead applied towards a more convenient microscopic analysis of smeared, fixed and stained RBCs. In this microscopy method, RCC samples are first diluted in plasma to reduce the hematocrit to physiological ranges of $40-45 \%$ [31]. The diluted samples are thinly smeared using the slide-to-slide technique [121] and then promptly fixed and stained using a Hema3 stain set [122]. The air-dried slides are viewed on a bright field microscope that is set up under the standardized Köhler illumination protocol [120]. Images are viewed under an oil-immersion $100 \times$ lens, and 100 cells are randomly chosen from 10 independent fields of view [89]. These cells are graded and assigned a factor that is provided for 
each RBC morphology feature (Section 1.5.3). The summation of factor values (Figure 1-16) for the 100 cells provides a morphology index (MI), that has been shown to decrease as a function of storage duration $[31,89]$.

\begin{tabular}{|c|c|}
\hline Description of RBC & Shape Factor \\
\hline Smooth Disc & 1.0 \\
\hline Crenated Disc & 0.8 \\
\hline Crenated Discoid & 0.6 \\
\hline Crenated Spheroid & 0.4 \\
\hline Crenated Sphere & 0.2 \\
\hline Smooth Sphere & 0.0 \\
\hline
\end{tabular}

Figure 1-16: Shape factor values assigned to morphology features of RBCs (1.5.3); values of 100 RBCs are summed to produce a morphology index (MI). Original method developed by Usry et al. [85].

\subsubsection{Spectrophotometry and Hemoglobin-Related Assays}

Spectrophotometry utilizes the principles of absorption spectroscopy to measure transmitted light emerging through a sample. If scanned over a range of wavelengths, spectrophotometers can generate sample spectra which can then be used to obtain quantitative parameters such as the concentration of the absorbing species (Section 1.6.2). For samples of RBCs, spectrophotometers can determine the concentrations of hemoglobin species (Section 1.2.2), and can derive other parameters such as the sample oxygenation (Section 1.2.3) and percent hemolysis (Section 1.4.1). With the aid of external attachments such as integrating spheres and monte carlo based simulation software, coefficients of absorption, scattering and anisotropy (Section 1.6.1) can also be calculated [123].

CBS routinely use spectrophotometers when determining percent hemolysis via Drabkin's cyanmethemoglobin $(\mathrm{HiCN})$ method. In this assay, Drabkins reagent is mixed with independent 
aliquots of RBCs and supernatant, which are extracted and separated from a sample of RCC [32]. Both aliquots are mixed with Drabkin's reagent, which lyses the RBCs and converts all hemoglobin into metHb (Section 1.2.2); furthermore, the cyanide reacts with metHb to form HiCN [124]. HiCN contains a distinct absorption peak at 540nm, that can be directly related to the concentration of hemoglobin [34]. Hence, by using a spectrophotometer, a ratio of supernatant-to-total hemoglobin concentration can be acquired, which translates to the percent hemolysis [28,124]. This spectrophotometric technique has been considered a gold standard in the monitoring of in vitro RBC hemolysis levels (Section 1.4.1) [125].

While spectrophotometers are used as a stand-alone instrument, the technology can be implemented into devices to add to their measuring capabilities. Considered a gold standard in hospital, blood bank and health care facilities, the blood gas analyzer (BGA) acquires several optical, electrolytic and metabolic-related RBC parameters [126]. Equipped with several electrodes, the BGA includes a spectrophotometer that determines the concentrations of functional and dysfunctional hemoglobin species as well as $\mathrm{SO}_{2}$ values [127]. The BGA has not only been a critical tool for data collection in clinical care, but also for the study of RBC storage lesions. For example, a BGA was used to monitor $\mathrm{pH}$ decrease and $\mathrm{pO}_{2} / \mathrm{SO}_{2}$ increase, as shown in Figure 1-8 and Figure 1-9 [56].

Spectrophotometers can also be used in conjunction with other technologies. For example, CBS and other blood bank laboratories use a Hemox-Analyser [128] to generate the ODC and determine the $\mathrm{p} 50$ (Section 1.2.3). The $\mathrm{pO}_{2}$ is increased and decreased by bubbling the sample with oxygen and nitrogen gas, respectively, while monitoring of this parameter is accomplished with an electrode [149]. $\mathrm{SO}_{2}$ is the y-axis of an ODC (Figure 1-4), hence it is monitored concurrently using an in-built spectrophotometer [129]. Since the p50 directly relates to the oxygen affinity of the sample hemoglobin, it is a useful variable for monitoring the effects of the $\mathrm{RBC}$ storage lesion on oxygen affinity. p50 values have shown to decrease over time in RCC samples stored in vitro [31]. 


\subsubsection{Flow Cytometry and RBC Fluorescent Staining Assays}

Flow cytometry is a sophisticated technology that has the capability of detecting multiple cell parameters in a large sample population with automation and high speed [130]. A typical flow cytometer contains three major functional systems: a hydraulic system that controls the alignment and passage of cells through a flow chamber; an optics system that includes a light source (comprising of at least one monochromatic laser) and typically two sensors; and an electronic system that separates and digitizes signals received from the sensors [131]. This digitized data can be statistically analyzed, generating quantitative measures on several cell features. If suitable features are plotted against each other, subpopulations can be easily distinguished amongst a heterogeneous sample [130].

First, cells are suspended in an isotonic fluid and transported to a flow chamber. As the sample enters the chamber, it is surrounded by a sheath fluid that confines the sample stream to the center of the chamber [132]. To ensure that the sheath fluid hydrodynamically focuses the sample stream, parameters such as pressure, center alignment and flow speed are monitored and maintained via a feedback loop [133]. These automated adjustments result in the sample stream flowing through the chamber one cell at a time [132]. Figure 1-17 illustrates the flow chamber setup.

Each cell in the sample stream intersects with the laser source(s) in the optics system. Light is scattered in a manner that is largely dependent on the cell size and intracellular contents [131]. Directionally speaking, the scattering can be categorized as either forward scatter $\left(0.5^{\circ}-10^{\circ}\right)$ or side scatter $\left(15^{\circ}-150^{\circ}\right)$ [137]. The tendency for light to be forward scattered is largely correlated with cell size. The intensity of side scattered light is correlated primarily with the internal heterogeneity, or granularity, of the cell [119]. In a cell containing high intracellular heterogeneity, light is prone to reflect and refract as it propagates across the cell, increasing the amount of side scattering events [134]. Hence, capturing signals from light that is forward scattered as well as side scattered can generate data that can be used to discriminate different cell subpopulations. 


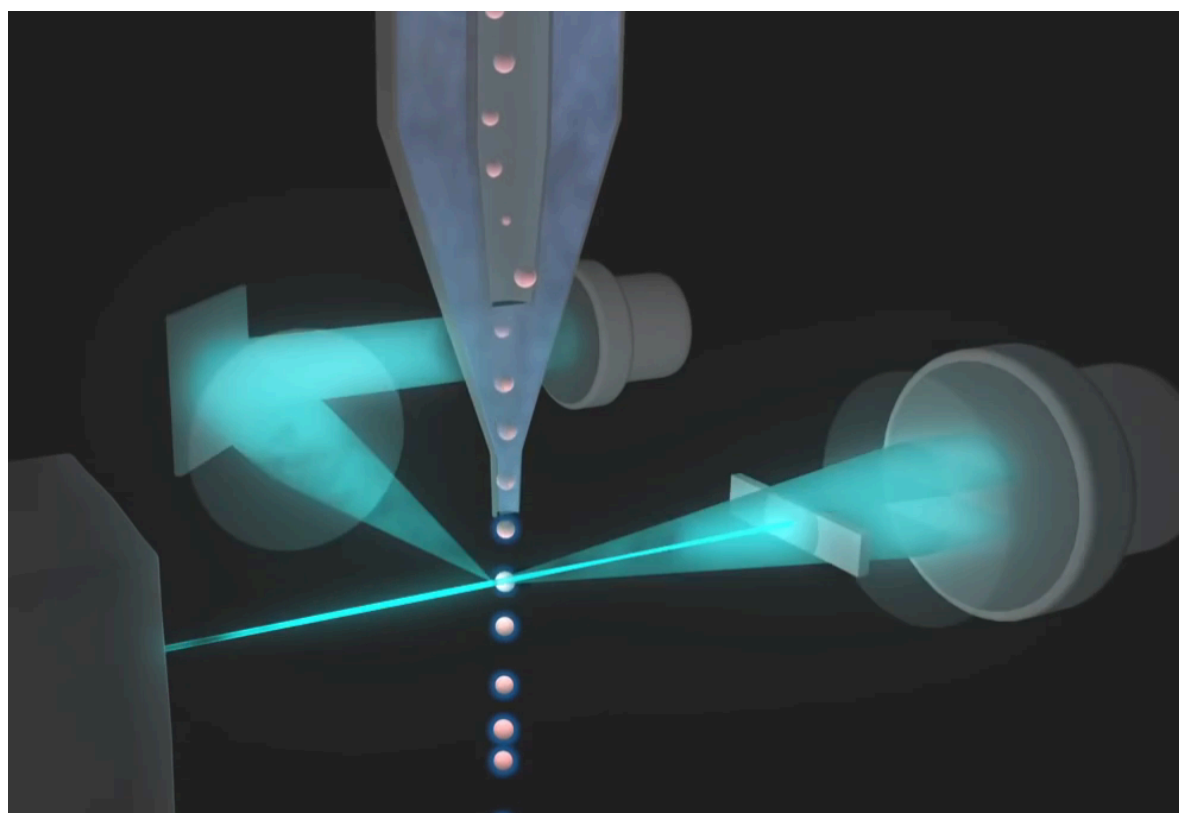

Figure 1-17: Simplified illustration of the optics (laser/PMT/photodiode) and hydraulic (sheath fluid/sample) setup of a modern flow cytometer.

In order to receive both forward and side scattered light, one sensor is positioned in line with the laser source, while the other sensor lies orthogonal to the laser direction. The orthogonal sensor is usually a photomultiplier tube (PMT) and is accompanied by a collection lens with a large numerical aperture [131]. This setup aids in capturing and amplifying the disperse side scattered light. On the other hand, the sensor in line of the direction of the laser receives a comparatively high intensity of light [119]. Beam absorbers and neutral density filters are often used to block excess light from entering the detector [131]. A collection lens guides forward scattered light towards a photodiode [131]. Figure 1-17 provides a schematic of the laser and sensor positions.

PMTs and photodiodes convert the incoming photons into voltage pulses that are proportional to the light intensity [119]. The pulses are processed by an analog-to-digital converter to digitize the quantity and intensity of signal. A frequency distribution plot can then provide evidence of certain characteristics of the sample population. For example, the illustration of the left of Figure 1-18 displays considerable variation in the size and granularity between subpopulations of WBCs. Upon observation, it can be said that lymphocytes are smaller in size and hence should exhibit the weakest forward scatter intensity. Furthermore, it can be speculated that a sequence of increasing granularity would group WBCs in the order of lymphocytes, monocytes and 
granulocytes. A dual parameter density plot of forward scatter and side scatter, shown on the right of Figure 1-18, exhibits relative values for each scatter that is consistent with the cell characteristics [131].

The intensities of forward and side scatter can infer relative measures of size and granularity, but these intrinsic signals cannot provide information on the actual contents of the cell. The technique of fluorescent marking, or cell staining, attempts to address this issue [135]. The quantum effect of fluorescence refers to the excitation of an atomic electron due to absorption of an incident photon, followed by the subsequent emission of a photon as the electron returns to its ground state. The emitted photon is of a longer wavelength, due to some energy being consumed by the absorbing electron in the form of thermal energy [130]. Furthermore, this photon is emitted in a direction that differs from the incident, and therefore fluorescence contributes to side scattering [119].

The ability to tag specific regions of the cell with fluorescent chemical compounds, known as fluorophores or fluorochromes, that have specific excitation and emission spectra, make fluorescence an extremely valuable technique in flow cytometry [130]. In a process known as staining, specific parts of a cell structure can be bound to fluorochromes, which could then be tracked by filtering for signal intensity at the expected emission wavelength range [135]. Furthermore, various parts of the cell can be stained with different fluorochromes that produce diverse excitation spectra. The electronics system can be equipped with multiple band pass filters and beam splitters so that each relevant wavelength range can be designated to a specific PMT [131]. These fluorescent signals, coupled with intrinsic forward and side scatter signals, provide opportunity for multi-parameter analysis.

The various cell morphologies of stored RBCs (Section 1.5.3) can result in side scattering due to a refractive index mismatch between the cell membrane and surrounding medium [119]. However, the lack of intracellular heterogeneity in an RBC results in highly forward scattering (Section 1.6.2). Forward scattering intensity cannot be directly linked to the size of an RBC. This is primarily due to the asymmetry of the biconcave disc, whose orientation can yield a misleading signal strength, indicating a falsely smaller cell size [119]. It is therefore impractical 

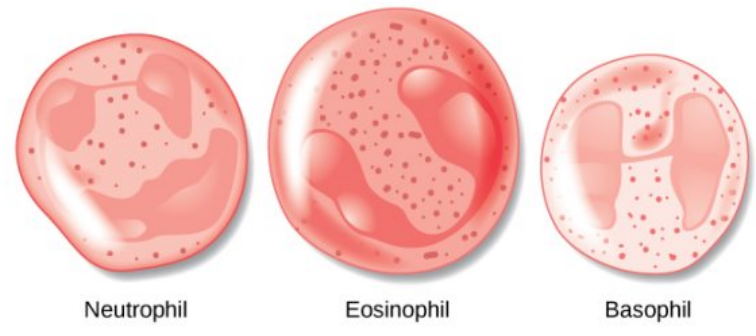

(a) Granulocytes
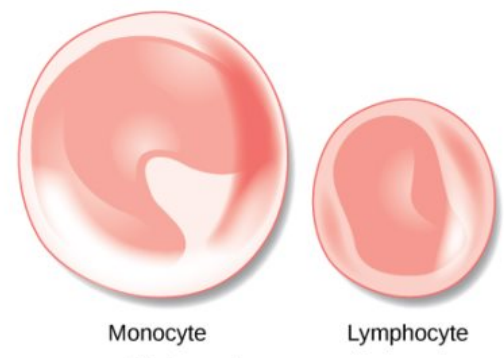

(b) Agranulocytes

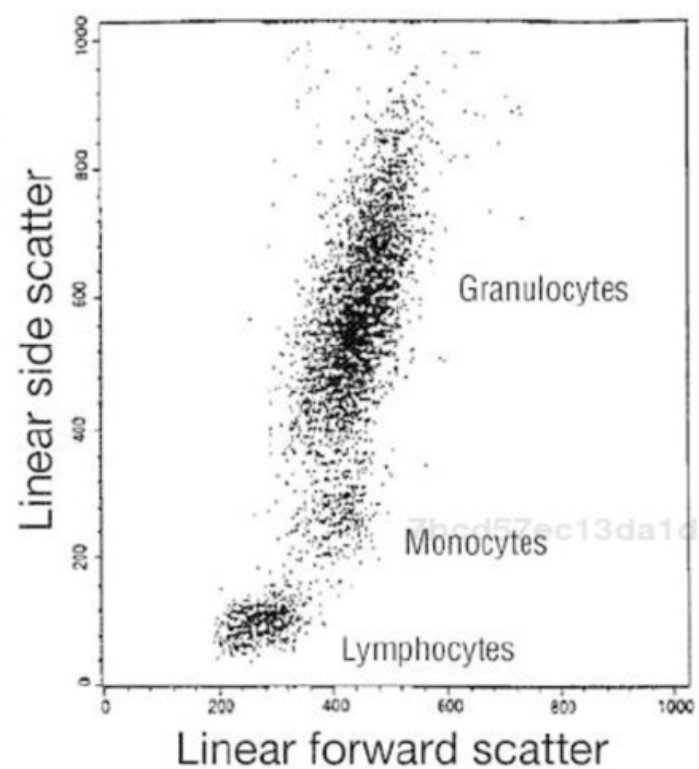

Figure 1-18: (left) An illustration of the subpopulations of WBCs (adapted from [138]); variations in cell size and granularity can be differentiated when (right) producing a dual parameter density plot of forward and side scatter intensities. Adapted from [131].

to characterize RBCs based on forward scatter alone. Under fluorescent staining however, RBCs are able to be distinguished more readily and can be examined for certain traits [131]. For example, RBCs have been stained for analysis of cell-bound proteins such as antibodies, which have resulted in important contributions to the field of immunohematology and transfusion science $[93,136,137]$.

\subsection{Introduction to Image Flow Cytometry (IFC)}

Image flow cytometry (IFC) is an optical technology that has been commercially available for just over a decade. In 2005, the first imaging flow cytometer was introduced by Amnis Corporation [139], with their latest upgrade in the technology offered in 2012 (named as Amnis Imagestream $^{\mathrm{X}}$ Mark II, or IS ${ }^{\mathrm{X}}$ : further details in Section 2.3). The novelty of IFC technology is in the incorporation of a transmitted light microscopy system into a flow cytometry setup, which results in the rapid acquisition of images [140]. Paired with a fully integrated image analysis 
package (IDEAS ${ }^{\circledR}$ software, Section 2.4), IFC expands on the analytical capabilities available in conventional flow cytometry (Section 1.7.3) while providing automation and speed that is impractical for conventional light microscopy (Section 1.7.1) [139].

By incorporating light microscopy into a flow cytometry platform, IFC provides the image resolution and morphological content of light microscopy, along with large sample size, automation and population statistics provided by flow cytometry. IFC incorporates a bright field light source to supply the necessary illumination for image capture. The light is magnified by a microscope objective lens and is spectrally decomposed before being guided towards high quality CCD cameras. The signals are spatially registered such that the intensity and the location of the signal can be displayed in the image [139]. IFC has the potential for image and population analysis that is unattainable using flow cytometry or light microscopy independently [140].

With the IFC system, researchers have analyzed brightfield (transmitted/forward scattered light), darkfield (side scattered light) and fluorescent microscopic images of different cell populations. Using various photometric and morphometric features, studies have been carried out on the modes of cell death [141], bone marrow cell formation pathways [142], and determining populations of circulating tumor cells [143]. Studies on RBCs have included the quantification of microvesicles in plasma [144], distinguishing stages of cell maturation [140] and detection of sickle cells among a sample of RBCs [145]. For example, Figure 1-19 displays a dual parameter plot that uses fluorescent and side scatter to distinguish cell subpopulations in whole blood, and displays some sample images acquired through brightfield, darkfield and fluorescence [144]. Through this work, IFC has been established as a powerful tool for the morphological characterization and analysis of cells by including the image resolution of microscopy and the speed of flow cytometry. 


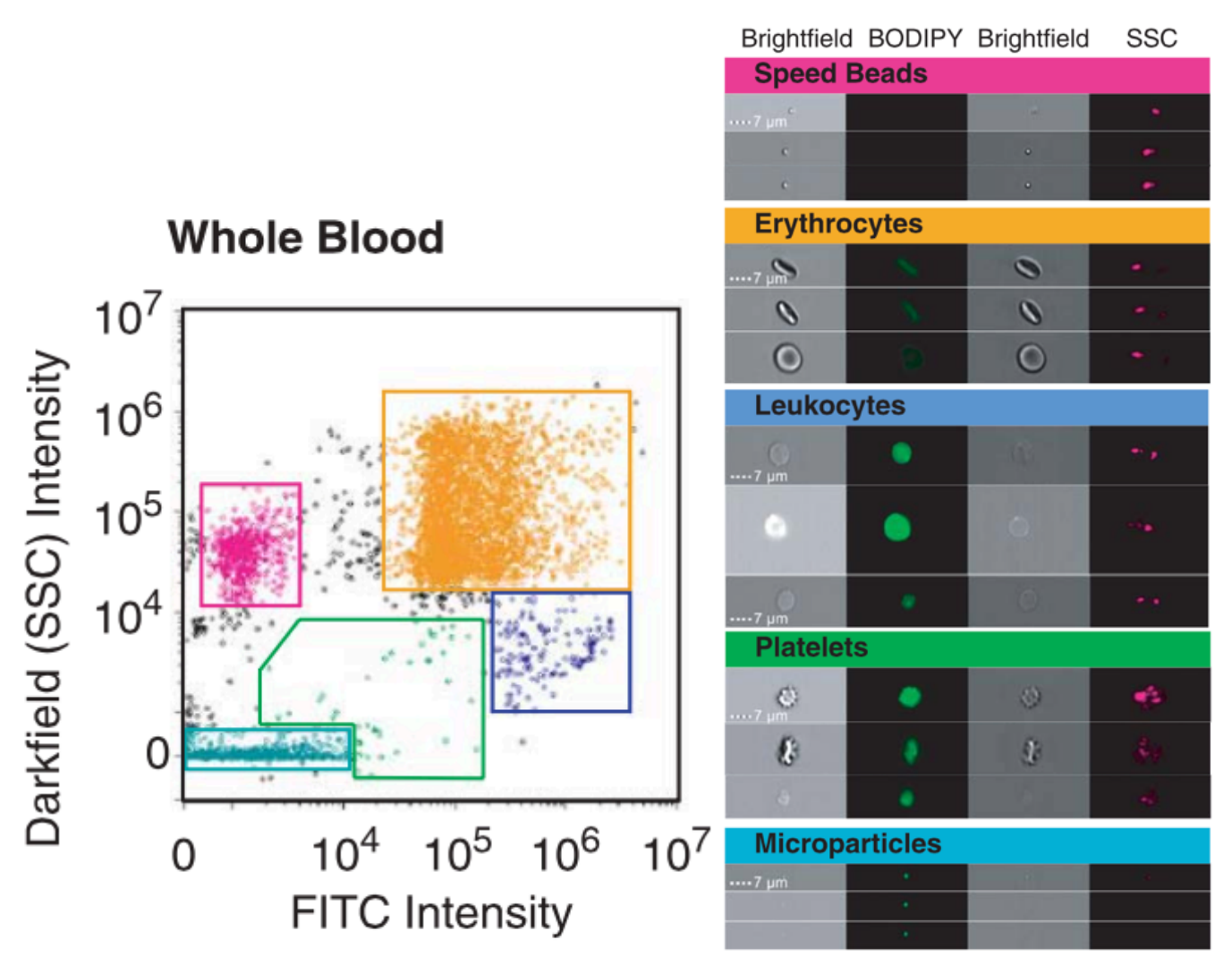

Figure 1-19: (Left) Plot of Darkfield intensity, or side scatter (SSC), against fluorescein isothiocyante dye (FITC) fluorescence intensity for calibration beads (pink), RBCs (orange), neutrophils (blue), platelets (green) and microvesicles, also known as microparticles (aqua). (Right) Brightfield images from two different CCD camera channels, BODIPY-Maleimide stained fluorescence images, and side scatter images of speed beads and components of whole blood. Images acquired simultaneously using Amnis IS ${ }^{\mathrm{x}}$ IFC. Adapted from [144].

\subsection{Introduction to Photoacoustics (PA)}

Photoacoustics (PA), alternatively known as optoacoustics, refers to the generation of acoustic waves due to the absorption of electromagnetic energy delivered by a light source. The PA effect was first reported by Alexander Graham Bell in 1880, who detected sound generated from thin selenium discs that were exposed to modulated sunlight [146]. Relatively little scientific progress was made until the invention of lasers and highly sensitive acoustic detectors, as well as the development of a theoretical description of the PA effect in solids [147]. By the mid-1990's, the potential of PA in biomedical imaging was being investigated, leading to the first PA images $[148,149]$. By the mid 2000's, compelling in vivo PA images were being obtained, increasing the scientific and clinical interest in the field over the last few decades [151-153]. 


\subsubsection{The PA Effect}

To generate a PA signal, light sources are delivered in either a short pulsed or continuous-wave modulation format. Short pulsed, laser-based sources provide a higher signal-to-noise ratio (SNR) and allow for time resolved detection of distances, making it the preferred source for biomedical applications [154]. The absorption of the pulse of photons raises the absorbing molecules to an excited state, which leads to either radiative relaxation (E.g. fluorescence) or non-radiative relaxation pathways [155]. In non-radiative relaxation, excitation energy can be transferred to neighboring molecules by increasing their kinetic energy through collisions [156]. The PA effect can only be formed from this pathway of non-radiative relaxation, since the molecular collisions lead to an increase in local temperature (in the order of millikelvins [157]). This rapid thermal motion or thermoelastic expansion translates to a longitudinal (pressure) wave that propagates outwards from the location of optical absorption.

For a PA signal to be generated, the laser pulse width (duration) must satisfy thermal and stress confinement conditions. Thermal confinement states that the pulse width $(\tau)$ must be short enough such that it can be assumed the thermal diffusion taking place during energy deposition is negligible [158]. The thermal confinement threshold $\left(\tau_{t h}\right)$ is given by:

$$
\tau<\tau_{\text {th }}=\frac{\mathrm{d}_{\mathrm{c}}^{2}}{4 \mathrm{D}_{\mathrm{T}}}
$$

where $d_{c}$ is the characteristic dimension of the region undergoing heating (i.e. absorber size) and $D_{T}$ is the thermal diffusivity of the sample [158]. With this condition satisfied, the PA pressure wave equation, as a function of spatial location $\vec{r}$ and time $t$ in a homogeneous, non-viscous medium is expressed as:

$$
\left(\nabla^{2}-\frac{1}{v_{s}^{2}} \frac{\partial^{2}}{\partial t^{2}}\right) p(\vec{r}, t)=-\frac{\beta}{C_{p}} \frac{\partial}{\partial t} H(\vec{r}, t)
$$

where $v_{s}\left[\frac{m}{s}\right]$ is the acoustic speed, $\beta\left[K^{-1}\right]$ is the isobaric volume expansion coefficient and $C_{p}\left[\frac{J}{K k g}\right]$ is the isobaric specific heat [158]. $H$ is a heating function that represents the amount of 
electromagnetic radiation that has converted into thermal energy, per unit volume and time. In the VIS-NIR optical regime, the heating function is represented by:

$$
H(\vec{r}, t)=\mu_{a}(\vec{r}) \cdot \Phi(\vec{r}, t)
$$

where $\mu_{\mathrm{a}}\left[\mathrm{m}^{-1}\right]$ is the absorption coefficient (Section 1.6.1) and $\Phi\left[\frac{\mathrm{J}}{\mathrm{m}^{2} \mathrm{~s}}\right]$ is the energy fluence rate [154].

Stress confinement is more restrictive in timescale than thermal confinement, and states that the pulse width must be short enough such that it can be assumed the thermoelastic expansion (or stress propagation) taking place during energy deposition is negligible. The stress confinement threshold $\left(\tau_{\text {st }}\right)$ is given by [157]:

$$
\tau<\tau_{\mathrm{st}}=\frac{\mathrm{d}_{\mathrm{c}}}{\mathrm{v}_{\mathrm{s}}}
$$

If both confinement conditions can be satisfied, the initial pressure (p) induced by laser excitation is given by:

$$
\mathrm{p}(\overrightarrow{\mathrm{r}})=\frac{\beta \mathrm{v}_{\mathrm{s}}^{2}}{\mathrm{C}_{\mathrm{p}}} \mu_{\mathrm{a}} \Phi(\overrightarrow{\mathrm{r}})=\Gamma \mu_{\mathrm{a}} \Phi(\overrightarrow{\mathrm{r}})
$$

where $\Gamma$ is termed as the Grüneisen parameter. Equation 1-14 establishes a proportionality between the amplitude of the generated pressure wave and the absorption coefficient, provided that confinement conditions are met.

Piezoelectric transducers can be used to detect the propagating acoustic signals. Using methods similar to pulse-echo distance calculations, signal processing algorithms can then be used to determine the spatial distribution of the sources generating the acoustic signals. This distribution is essentially a map of the energy deposition that generated the pressure waves, and therefore provides an image of the absorption profile of the illuminated target. 


\subsubsection{Advantages over Conventional Optical/Ultrasound Technologies}

Optical technologies are limited by poor penetration depth, especially when illuminating a sample of RCC that contain a high concentration of strong chromophores. PA technology has the potential to overcome this limitation, as it receives acoustic rather than optical signals which encounter 2-3 orders of magnitude less scattering [159]. Although both ultrasound (US) and PA use acoustic signals to produce images, the contrast mechanisms between the two technologies differ. US signals are a measure of the acoustic impedance mismatches between different boundaries within a sample, and are dependent on elastic and mechanical properties [159]. On the other hand, PA signals measure the optical energy absorption profile of the target. Hence, PA can provide more detail when imaging targets that possess large optical absorption but low echogenicity [159]. Furthermore, since absorption is a wavelength-dependent phenomenon (Section 1.6.1), PA can acquire spectroscopic information of the absorbing species [157].

Unlike purely optical technologies, PA can potentially acquire morphologically related information of the absorbing species by analyzing the frequency content of the acoustic signal. For example, photoacoustic radio-frequency spectroscopy (PARFS) has been used to characterize the morphology of individual red blood cells $[160,161]$ and study RBC aggregation [162]. This added capability to infer structure from the frequency data gives PA an added advantage compared to other optical methods.

\subsubsection{PA Oximetry}

The absorption coefficient of a medium is proportional to with the measured absorbance of that medium (Equation B2, Appendices B); in PA, the absorption coefficient is also linked with the pressure amplitude of the generated acoustic wave (Equation 1-14). Hence, the spectroscopic capabilities of PA can be extended to oximetry (Section 1.6.3). Assuming that changes in the fluence profiles between wavelengths is negligible, absorbance can be replaced by photoacoustic pressure amplitude in Equation 1-8, yielding the following expression for $\mathrm{SO}_{2}$ : 


$$
\mathrm{SO}_{2}=\frac{\Phi^{\lambda_{2}} \cdot \varepsilon_{\mathrm{Hb}}^{\lambda_{1}}-\Phi^{\lambda_{1}} \cdot \varepsilon_{\mathrm{Hb}}^{\lambda_{2}}}{\Phi^{\lambda_{1}} \cdot \Delta \varepsilon^{\lambda_{2}}-\Phi^{\lambda_{2}} \cdot \Delta \varepsilon^{\lambda_{1}}}
$$

where the symbol $\Phi^{\lambda}$ represents the PA signal amplitude at illumination wavelength $\lambda$, and $\Delta \varepsilon^{\lambda}$ is given by Equation 1-9 [163].

\subsubsection{Potential for non-invasive $\mathrm{SO}_{2}$ Acquisition}

In the wavelength region of approximately $600-1100 \mathrm{~nm}$, non-radiative relaxation is dominated by thermalization [159], allowing for PA signals to be efficiently produced under thermal and stress confinement (Section 1.9.1). Light at this range is primarily absorbed by hemoglobin, however, the absorption coefficients are minimized (Figures 1-13, 1-15), facilitating deeper penetration of light. In this range the pulsed electromagnetic radiation is non-ionizing and produces temperature increases of a few millikelvins; hence, molecular damage or alteration is not of concern in PA. These factors allow the opportunity to use PA within this "optical window" for in vivo applications.

PA imaging has been used to detect/image in vivo tumor vasculature [151], prostate cancer [164] and circulating tumor cells [165]. Blood flow and flow characteristics of RBCs have been studied by employing ultrasound doppler techniques [166,167]. Using the principles of oximetry, numerous studies have simultaneously measured blood oxygenation $\left(\mathrm{SO}_{2}\right)$ to provide contrast and functional information of vascular structures non-invasively [152,153,168,169]. The prevalence of $\mathrm{PA} \mathrm{SO}_{2}$ acquisition has led to the generation of real-time $\mathrm{SO}_{2}$ mapping in many PA devices (Section 2.5).

\subsection{Thesis Motivation}

In Canada, approximately 850,000 units of blood are collected and transfused into thousands of patients annually. Red blood cells (RBCs) are the most frequently transfused constituent of blood because it augments the blood volume and oxygenation in an individual. 
Under Health Canada/CBS guidelines, RBCs are packaged and stored in a hypothermic temperature $\left(1-6^{\circ} \mathrm{C}\right)$ and are considered transfusable for a period of 42 days.

During storage, significant alterations in the structure and function of RBCs have been documented (Section 1.5). RBC morphology and oxygen saturation $\left(\mathrm{SO}_{2}\right)$ are examples of structural and functional changes, respectively, that occur during storage. These and other changes lead to an irreversible structural and functional degradation of the cell that are collectively termed as RBC storage lesions.

Growing evidence suggests that RBC storage lesions may increase patient morbidity in patient transfusions, especially in vulnerable patient populations. Currently, no technique or technology exists that can rapidly characterize the overall quality of RBCs pre-transfusion; the introduction of such a quality assurance tool could aid in minimizing post-transfusion complications.

Optical technologies are well suited for the characterization of RBCs, due to strong optical absorption of the hemoglobin in RBCs, as well as unique spectral features of hemoglobin that exist in the VIS-NIR optical window. Several technologies have been developed to exploit the optical properties of RBCs and infer their morphological and functional state. Current RBC morphology assays involving light microscopy are labor intensive and are operator dependent (and therefore subjective). Furthermore, the assays require chemical preparations and smearing of cells that could introduce artifacts and alter the in vitro structural state of RBCs. Current $\mathrm{SO}_{2}$ assays involving absorption spectroscopy require the extraction of RBCs from its storage environment, causing unwanted external exposure that may influence the in vitro functional state of RBCs.

Image flow cytometry (IFC) is a decade-old optical technology that combines the high throughput of flow cytometry with the resolution of light microscopy. The output is a large collection of images that can be analyzed using a suitable image analysis software package. IFC is ideal for RBC morphology characterization, as it measures cell structure in an isotonic fluid suspension by generating high resolution brightfield images outlining RBC shape. An 
opportunity exists for the development of a novel, rapid and automated RBC morphology assay. At the time of submission of this thesis and to the best of the author's knowledge, there has been no published work on rapid assessment of RBC morphology assay using IFC technology.

Photoacoustic (PA) technology uses a pulsed laser source directed at an absorbing species to produce acoustic waves that are detected using passive transducers. PA is well suited for the non-invasive, spectroscopic monitoring of RBCs as the PA signal amplitude is proportional to optical absorption. Furthermore, PA can use the 2-wavelength oximetry principle approach to acquire the $\mathrm{SO}_{2}$ of an illuminated sample of RBCs. PA can potentially be used to monitor the $\mathrm{SO}_{2}$ of RBCs within their storage environment (blood bags), as well as acquire parameters related to RBC morphology. At the time of submission of this thesis and to the best of the author's knowledge, there has been no published work on a PA methodology to monitor $\mathrm{SO}_{2}$ of RBCs in blood bags in situ.

RBCs storage lesion morphology can be parametrized by recording the percentage of spheroechinocytes in the RBC population which increase over the storage duration. The depletion of in vitro 2,3-DPG concentration, which is an oxygen-hemoglobin binding regulator, is expected to increase $\mathrm{SO}_{2}$ measurements of the stored blood over the storage duration. It is plausible that the both RBC storage lesion parameters - spheroechinocyte population percentage and $\mathrm{SO}_{2}$ percentage - are correlated over time. If there is such a correlation, in situ monitoring of $\mathrm{SO}_{2}$ changes could then be used to infer the changes in the morphological state of RBCs. This would result in a rapid $\mathrm{PA}$ technique for the monitoring of $\mathrm{RBC}$ storage lesions passively (without sample extraction).

\subsection{Hypothesis and Specific Aims}

Two hypotheses will be examined in this thesis, which are designated as a primary hypothesis and secondary hypothesis.

Primary Hypothesis: 
During blood bag storage, changes in the RBC morphology distribution are correlated with changes in $\mathrm{RBC}$ oxygen saturation $\left(\mathrm{SO}_{2}\right)$.

\section{Significance:}

If a correlation is established, the RBC storage lesion population changes can be monitored through $\mathrm{RBC} \mathrm{SO}_{2}$ changes.

Specific Aims:

1) Design an RBC morphology characterization technique using image flow cytometry, to determine the spheroechinocyte population percentage for a sample of RCC.

2) Implement an $\mathrm{RCC} \mathrm{SO}_{2}$ acquisition protocol using the conventional method of blood gas analysis.

3) Design an experimental protocol for the comparison of blood gas analysis $\mathrm{SO}_{2}$ results and image flow cytometry measurements of RBC morphology with the same RCC samples.

Secondary Hypothesis:

Photoacoustic technology can be used to acquire the $\mathrm{SO}_{2}$ of RBCs in a blood bag in situ .

\section{Significance:}

Non-invasively measuring the $\mathrm{SO}_{2}$ values of blood in situ (in a blood bag) will enable measurements without the need for sample extraction.

Specific Aims:

1) Develop an $\mathrm{SO}_{2}$ acquisition method of $\mathrm{RCC}$ in blood bags using photoacoustic technology.

2) Design an experimental setup for consistent photoacoustic measurements of RCC in blood bags.

3) Create an experimental protocol for $\mathrm{SO}_{2}$ validation of in situ photoacoustic measurements against blood gas analysis. 


\section{Chapter 2 : Materials and Methods}

The materials section of this chapter begins with a description of the blood products used for all thesis experiments (Section 2.1). Section 2.2 introduces the Blood Gas Analyser (BGA), as well as associated sample syringes and spectrophotometric setup used for $\mathrm{SO}_{2}$ acquisition. Section 2.3 introduces the components making up the image flow cytometry (IFC) system. Section 2.4 introduces the image analysis software program and the general parameters used to develop the RBC morphology image segmentation template. Section 2.5 presents the components associated with the photoacoustic (PA) system.

The methods section of this chapter begin with the description of two control experiments (Section 2.6). The first experiment investigates if the blood bag plastic produces a PA signal; the second investigates, for varied ranges of oxygen saturation, if the $\mathrm{PA} \mathrm{SO}_{2}$ of hemoglobin covered by blood bag plastic can agree with BGA measurements. Section 2.7 describes all three (IFC, BGA and PA) experimental setups and protocols for time series data collection. Section 2.8 briefly explains the computational software used for data storage and graphing, as well as statistical techniques used to test the thesis hypotheses.

\subsection{Blood Products and Storage}

\subsubsection{Lyophilized Hemoglobin}

Control experiments (Section 2.6) were carried out using human lyophilized (powder) hemoglobin (Sigma-Aldrich, MO, USA). The hemoglobin samples were mostly (approximately $85 \%$ ) in metHb form (Section 1.2 .2 ), with a solubility of $20 \mathrm{mg} / \mathrm{ml}$ in water [170].

\subsubsection{CBS Blood (RCC) Bags}

Seven RCC blood bags were transported from Canadian Blood Services (CBS) blood-forresearch center (netCAD, Vancouver, Canada) to the Institute of Biomedical Engineering, Science and Technology (iBEST, Toronto, Canada). Throughout the 15-20 hour transit period, 
the bags were insulated in standard blood shipment containers which are comprised of a styrofoam/cardboard box filled filled with ice packs to maintain hypothermic temperatures. The RCC provided in blood bags were separated from anticoagulated whole blood and leukoreduced via the BC method (Section 1.3.3), and stored in up to $110 \mathrm{ml}$ of SAGM preservative solution. Upon reception, blood bags were promptly stored in a designated laboratory fridge until six weeks after the production date. Before the first extraction of the sample for IFC analysis, bags were fitted with a sampling site coupler that contained a natural rubber sleeve stopper for needle penetration.

\subsection{Radiometer ABL800 FLEX ${ }^{\circledR}$ Blood Gas Analyzer}

Blood Gas Analyzers (BGA) are considered the gold standard in blood sample analysis within the healthcare environment, providing critical information that is used for diagnosis and treatment [126]. BGAs underwent significant adaptations in technology in the mid 2000's, allowing for the acquisition of more than $\mathrm{pH}$ and blood gas parameters [171]. Advances in sample handling, auto-calibration and quality management have expanded the BGA measurements to include parameters associated with hemoglobin, as well as various blood metabolites and electrolytes [172]. While BGAs are found in most clinical laboratories, portable benchtop BGAs have recently emerged in critical care locations such as operating rooms, intensive care units and emergency departments [173]. Hence, BGAs have enabled bedside or point of care (POC) in vitro analysis that allows for more timely decisions and enhancing patient management [126].

Radiometer, a major manufacturer of BGA devices, recently introduced the ABL800 FLEX (FLEX for short) and was the first instrument to automate blood sample handling [176]. The design reduces manual steps and preanalytical errors that commonly affect the accuracy and precision of results [126]. The FLEX can measure up to 18 analytes rapidly, and can do so by mixing, measuring and storing the values of up to three samples in automated succession. Table 2-1 provides a list of the parameters available with the FLEX [126]. The following sections present the methods and techniques used by FLEX for blood sample handling and spectroscopic analysis of hemoglobin. 


\begin{tabular}{|c|c|c|c|c|c|c|}
\hline \multirow[b]{2}{*}{ Manufacturer } & \multirow[b]{2}{*}{ Model } & \multirow[b]{2}{*}{ Category } & \multicolumn{4}{|c|}{ Measured Analytes } \\
\hline & & & Blood Gas & Electrolytes & Metabolites and Other Tests & CO-Oximetry \\
\hline Radiometer & ABL 800 FLEX & $\begin{array}{l}\mathrm{POC} \text { and } \\
\text { centralized } \\
\text { testing }\end{array}$ & $\begin{array}{l}\mathrm{pH}, \mathrm{pCO}_{2} \\
\mathrm{pO}_{2}\end{array}$ & $\begin{array}{l}\mathrm{Na}^{+}, \mathrm{K}+ \\
\mathrm{Ca}^{+}, \mathrm{Cl}^{-}\end{array}$ & $\begin{array}{l}\text { Glucose, lactate, creatinine, } \\
\text { bilirubin }\end{array}$ & $\begin{array}{l}\mathrm{tHb}, \mathrm{HHb}, \mathrm{O}_{2} \mathrm{Hb}, \mathrm{sO}_{2}, \\
\mathrm{COHb}, \mathrm{MetHb}\end{array}$ \\
\hline
\end{tabular}

Table 2-1: List of the analytes available for measurement using the Radiometer ABL800 FLEX. Table adapted and modified from [126].

\subsection{1 safePICO Aspirator Syringe}

A syringe manufactured by Radiometer, called safePICO, is designed with the aim of enhancing patient and operator safety, while reducing preanalytical errors [175]. The syringes are barcoded for automated tracking and linking with stored data. They are also pre-heparinized to reduce risks of clots or electrolyte bias before measurement. safePICO syringes are fitted with a custom designed cap (safeTIPCAP) that is vented one way for the expulsion of air within the syringe, while sealing the sample from the external environment [175]. The technology conveniently removes air bubbles/pockets within the extracted blood sample that could influence gaseous parameters values such as partial pressures and $\mathrm{SO}_{2}$.

The automated sample handling feature of the ABL involves the manual insertion of safePICO syringes into one of three slots; the safeTIPCAP cover is left on the syringe. Firstly, a scanner reads the barcode of the syringe. An inert gold plated mixing ball is moved by a rotating magnet for 7 seconds, providing a consistent technique of mixing prior to measurement [175]. An inlet probe enters the syringe through the safeTIPCAP to aspirate $195 \mu$ of sample. After approximately one minute, a digital and hard copy of parameter values are provided. Figure 2-1 displays the syringe and its components; Table A-1 in the Appendices provides more details on the safePICO syringe [174,175]. 


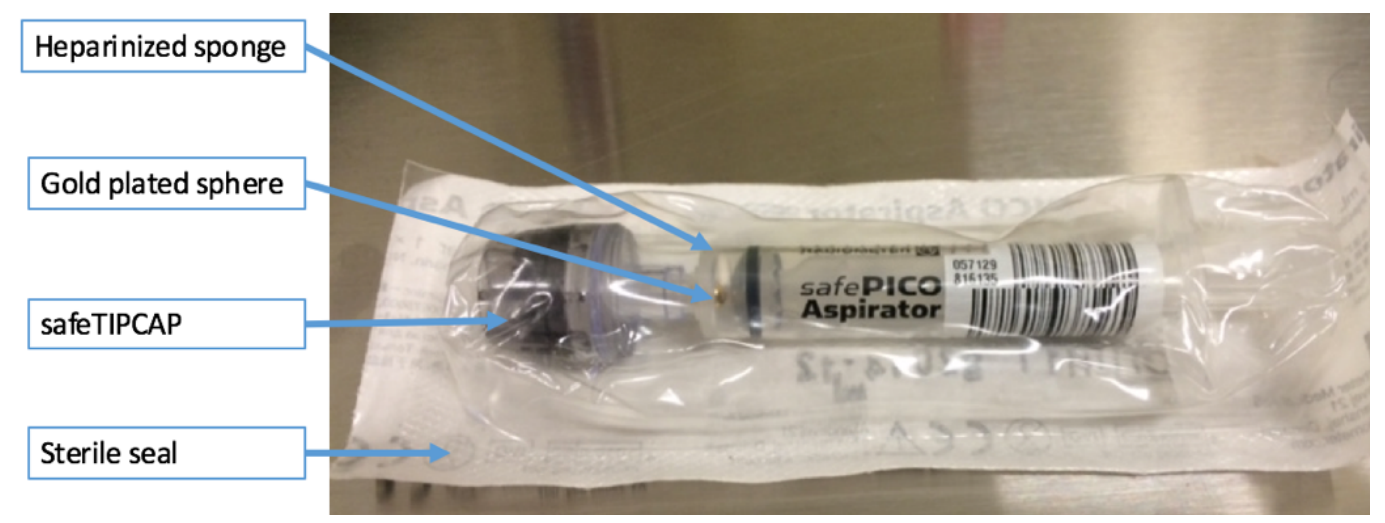

Figure 2-1: The safePICO Aspirator and components within and encapsulating the syringe.

\subsubsection{Optical System and Spectroscopic Measurement}

The optical system of the FLEX uses the principles of visible absorption spectroscopy (Section 1.6.2) with a 128-wavelength spectrometer to acquire parameters such as $\mathrm{SO}_{2}$, total hemoglobin concentration and fractional concentrations of hemoglobin states [176]. Once the blood sample has been aspirated into the FLEX, it is transported into a quartz glass cuvette that is regulated at $37^{\circ} \mathrm{C}$ and $1 \mathrm{~atm}$ pressure. $1 \mu \mathrm{l}$ of the sample is hemolyzed with $30 \mathrm{kHz}$ ultrasound vibrations, releasing the hemoglobin into the supernatant.

Light is provided by a $4 \mathrm{~W}$ halogen lamp that is infrared-filtered and focused before entering the cuvette. The transmitted light is guided by an optical fiber into the spectrophotometer, where it separated by a concave grating into monochromatic signals between 478 and $672 \mathrm{~nm}$. These 128 signals are focused onto a photodiode array that dedicates one pixel for each wavelength. Figure 2-2 provides a schematic of the optics-spectrometer system.

The absorption spectrum generated from the intensity contributions of each signal is used to estimate the concentrations of the absorbing species, which is assumed to be oxyHb, deoxyHb, metHb and $\mathrm{COHb}$ (Section 1.2.2). For the FLEX spectroscopic setup, Equation 1-4 can be expressed as: 


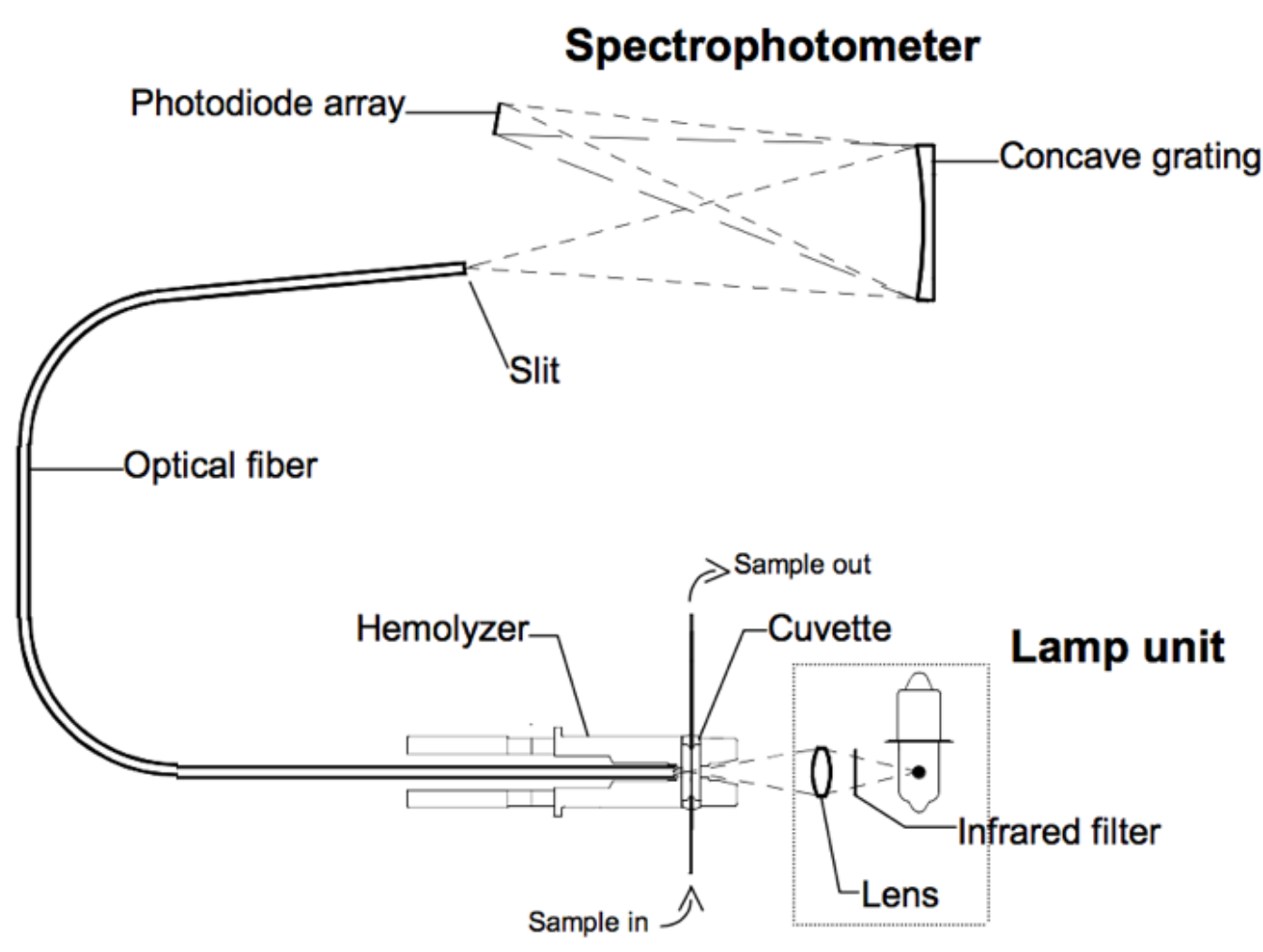

Figure 2-2: The optics (below) and spectrometer (above) compartments of the ABL800 FLEX BGA. Adapted from [176].

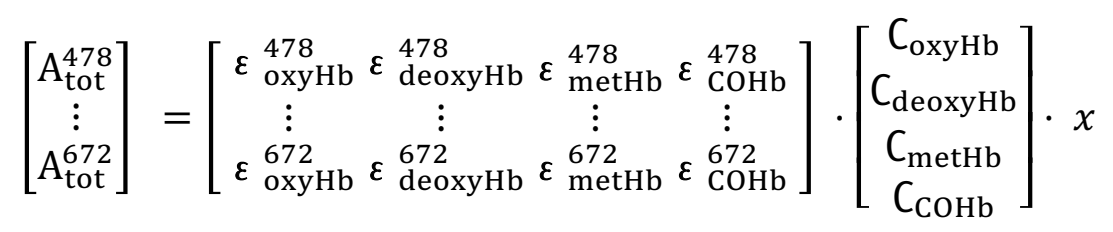

however, correction factors need to be incorporated into Equation 2-1. Reference values of proprietary calibration compounds were combined with the path length and extinction coefficients of each state of hemoglobin to form a matrix of proprietary constants $\left(K^{\lambda}\right)$ that result in the following form of Equation 2-1 [176],

$$
C_{y}=\sum_{\lambda=478}^{672} K_{y}^{\lambda} \cdot A_{\text {tot }}^{\lambda}
$$


where $C_{y}$ represents the concentration of absorbing species, and the right hand side of Equation 2-2 is solved using Multivariate Data Analysis [177]. Once the concentrations are determined, total hemoglobin concentration and $\mathrm{SO}_{2}$ are calculated as:

$$
\begin{aligned}
& \text { Total hemoglobin concentration }=C_{\text {oxyHb }}+C_{\text {deoxyHb }}+C_{\text {metHb }}+C_{\mathrm{COHb}} \quad[2-3] \\
& \mathrm{SO}_{2}=\frac{\mathrm{C}_{\text {oxyHb }}}{\mathrm{C}_{\text {oxyHb }}+C_{\text {deoxyHb }}}
\end{aligned}
$$

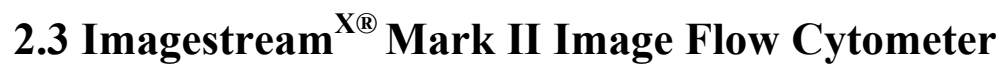

The Imagestream $^{\mathrm{X} \circledast}$ Mark II (IS ${ }^{\mathrm{X}}$ for short) image flow cytometer (IFC) provided by MilliporeSigma (parent company of Amnis Corporation) came into the market in 2012 as the latest upgraded device in IFC technology [178]. IFC incorporates light microscopy (Section 1.7.1) into a traditional flow cytometry setup (Section 1.7.3), creating the unique ability to capture numerous brightfield, darkfield and fluorescent images at high speed. In order to merge two well-established optical technologies into one platform, novel optical and electronic techniques have been incorporated. Some of the major features and techniques behind the ${ }^{\mathrm{X}}{ }^{\mathrm{X}}$ will be presented in the following sections.

\subsubsection{IS ${ }^{\mathrm{X}}$ Platform}

As in conventional flow cytometry (Section 1.7.3), cells are suspended in an isotonic fluid before being inserted into the $\mathrm{IS}^{\mathrm{X}}$ and are hydrodynamically focused using a phosphate buffered saline (PBS) sheath fluid [205]. A single-file sample stream of approximately $10 \mu \mathrm{m}$ diameter enters the flow cell, upon which each cell is intersected by the light source [133]. Unlike a typical flow cytometry system that involves two detectors placed in line with and orthogonal to a laser source (Figure 1-17), the IS ${ }^{\mathrm{X}}$ contains a brightfield and laser light source placed, respectively, in line with and orthogonal to one microscope objective (Figure 2-3). It is necessary to have this orientation for the desired transmitted, scattered and fluorescence light to travel through the collection lens (Section 1.7.3). 


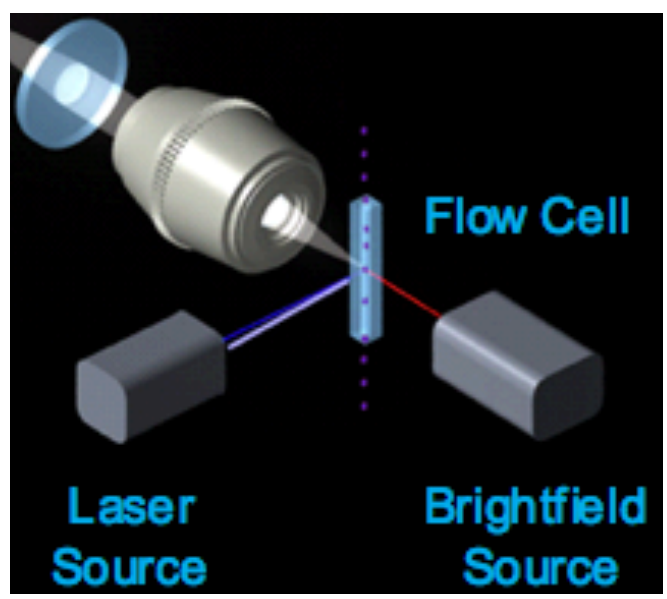

Figure 2-3: Illustration of the orientation of the flow cell, light sources and microscope objective in the IS ${ }^{\mathrm{X}}$. Adapted from [179].

Figure 2-4 displays an aerial-view schematic of the IS $^{\mathrm{X}}$ optics platform. Although not displayed in the figure, light from an $830 \mathrm{~nm}$ laser intersects calibration beads injected into the sheath fluid stream and is scattered and into a flow speed and autofocus detection system [179]. The autofocus system continuously maintains the alignment of the core stream so that cells traverse through the focal regions of the illumination beams [133]. The flow speed detection system is used to maintain synchronization with the camera system by adjusting the speed of the fluid stream (Section 2.3.2).

The transmitted, scattered and fluorescent light is directed towards a multi-spectral decomposition unit (MSDU) (Figure 2-4). The MSDU consists of several low-pass dichroic mirrors oriented at specific angles that separate the incoming light beam into spectral bands [133]. Furthermore, these spectral bands are deflected by the MSDU at different angles and consequently project onto the CCD camera at different lateral positions [141]. For each camera, six image channels are available to project the signals of different spectral bands, allowing for the capability to use a multitude of fluorescent tags for measurements [178]. Two channels are used by default for bright field images (and can be changed by the user), while one channel is used for side scatter images produced from a 785nm laser. Table A-2 in the Appendices provides further information on the spectral ranges of each channel, as well as other features of the IS ${ }^{\mathrm{X}}$. 


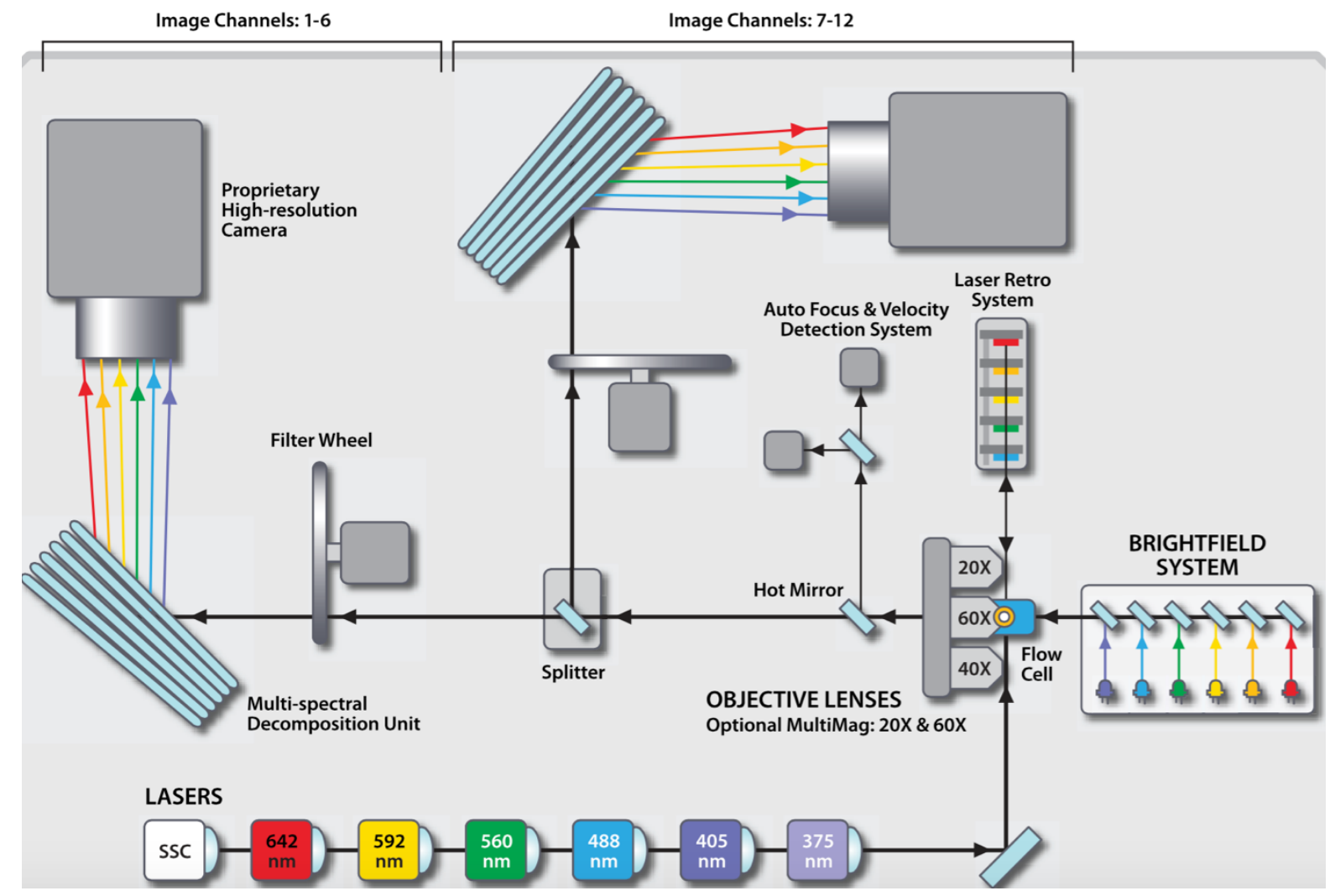

Figure 2-4: Aerial-view diagram outlining the optics platform of the IS ${ }^{\mathrm{X}}$. Note that SSC is an abbreviation for side scatter 785nm laser. Adapted from [178].

\subsubsection{Optimizing Image Intensity}

Unlike conventional flow cytometers that use PMTs to amplify the signal intensity of weakly scattered light (Section 1.7.3), the $\mathrm{IS}^{\mathrm{X}}$ needs to preserve spatial information of the signal and therefore requires a different method to increase the sensitivity of detection. IS ${ }^{\mathrm{X}}$ employs time delay integration (TDI), a technique commonly used in applications where the object being imaged moves fast relative to the camera [141]. In TDI, the image projected onto the detector is read out from the bottom pixel row, and the upper pixel row intensities are transferred one row down. This readout/shift rate is synchronized with the flow speed of the sample cells with the help of a flow speed detection system (Section 2.3.1). Synchronization preserves the sensitivity and image quality, while increasing the intensity of the image as it translates down the CCD detector before readout [162]. Figure 2-5 provides an illustration of the TDI method and the increase in image intensity due to synchronization [205]. 


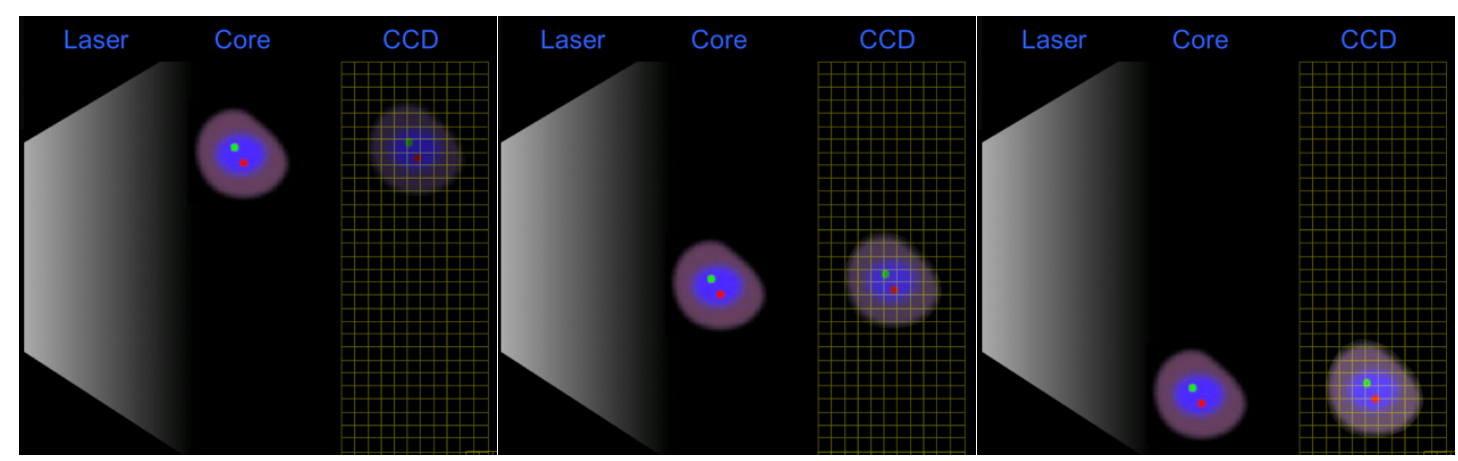

Figure 2-5: Illustration of the TDI method of enhancing intensity of image. Each frame consists of (from left to right) the illumination source, cell motion in the sample stream and projection of the corresponding image on a CCD detector. Adapted from [179].

The TDI method integrates the signal from each cell for around 10 milliseconds, depending on the flow speed of the sample [153]. This provides an opportunity for otherwise faint fluorescent and scattered signals to be detected at high flow speeds [180]. Cell alignment consistency is essential for TDI to enhance image quality. IS $^{\mathrm{X}}$ employs optimized precision stepper motor pumps and an autofocus detection system (Section 2.3.1) to reduce pulsatility [133]. Consequently, highly laminar flow is produced that reduces cell tumbling and alignment deviations during image acquisition [180].

\subsection{Amnis IDEAS ${ }^{\circledR}$ Analysis Software}

Conventional flow cytometry (Section 1.7.3) is paired with software to objectively and statistically discriminate populations of cells based on signal intensities. With the IS ${ }^{\mathrm{X}}$ IFC however, this objective, statistical discrimination can be applied to photometric, and additionally, morphometric parameters. An image analysis software called IDEAS ${ }^{\circledR}$ has been integrated with the IS $^{\mathrm{X}}$ for image analysis and population statistics. The following sections introduce the basic software platform and tools used for the development of the RBC morphology image segmentation template.

\subsubsection{IDEAS ${ }^{\circledR}$ File Formatting for Analysis}

After TDI (Section 2.3.2) is applied, the intensity values registered for each pixel in a particular channel are plotted as the third variable on a two dimensional grid [179]. As Figure 2-6 


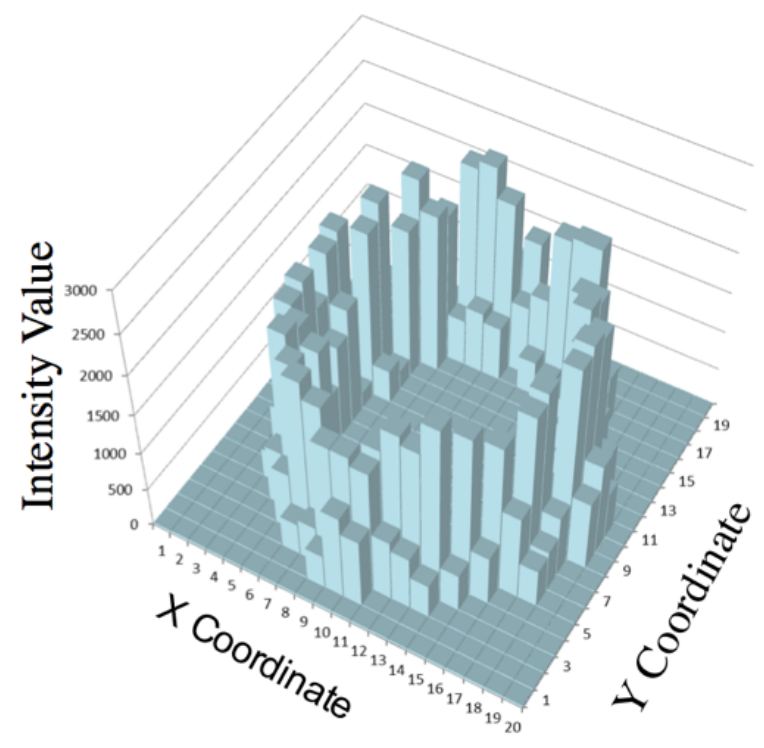

Figure 2-6: Plot of intensity values on a two dimensional grid representing location of pixels on CCD detector. Photometric and Morphometric information is encoded into such plots. Adapted from [178].

illustrates, this plot displays photometric information via intensity peaks and morphometric information via spatial distribution. On $\operatorname{IDEAS}^{\circledR}$, the intensity values acquired in each spectral channel translate to a standard grey-scale image, with spatial scales directly related to the dimensions of the pixels and magnification of the image.

The pixel intensity data for each detected object is saved by the $\operatorname{IS}^{\mathrm{X}}$ application program, INSPIRE, as a raw image file (.rif) [181]. The IDEAS $^{\circledR}$ [207] software application uses the .rif file to create a compensated image file (.cif) that corrects data for variations in background intensity, flow speed, position variations between channels and other artifacts. This “compensated" image data file requires a template file to be used for analysis on IDEAS ${ }^{\circledR}$. The .cif file is loaded into a default template file (.ast); the resulting data analysis file (.daf) opens an interface on IDEAS $^{\circledR}$ for direct manipulation.

The .daf file can be custom structured to include features, graphs, regions of interest (ROIs) and statistics. This file can be saved as a ast file to serve as a new, customized template that can be applied to any .cif files to create their associated daf files. Hence, image and statistical analysis of experimental data using IDEAS $^{\circledR}$ starts with creating a suitable template file. Figure 2-7 


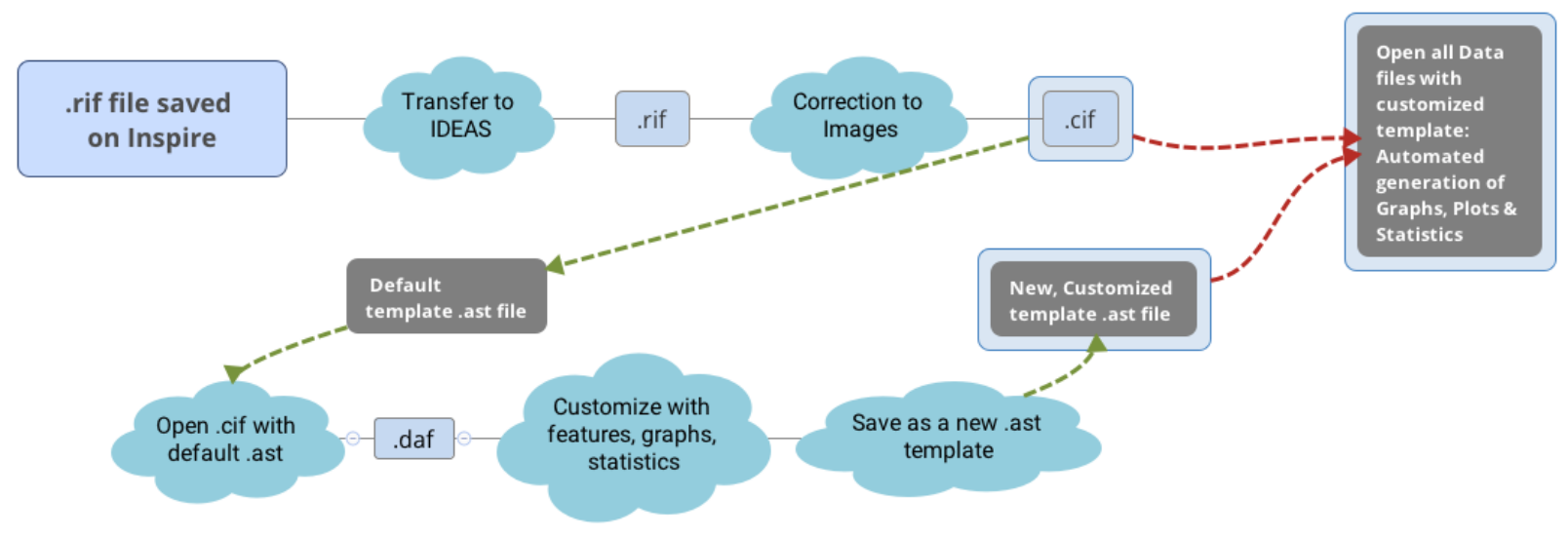

Figure 2-7: Schematic representation of the IDEAS ${ }^{\circledR}$ file format process flow to obtain a customized template for automated analysis.

displays a schematic representation of the file format process flow for generating an IDEAS ${ }^{\circledR}$ template for automated analysis. Figure 2-8 provides the layout of a .daf file that was opened with a template file designed for the automated analysis of a sample of RBCs (Section 3.2).

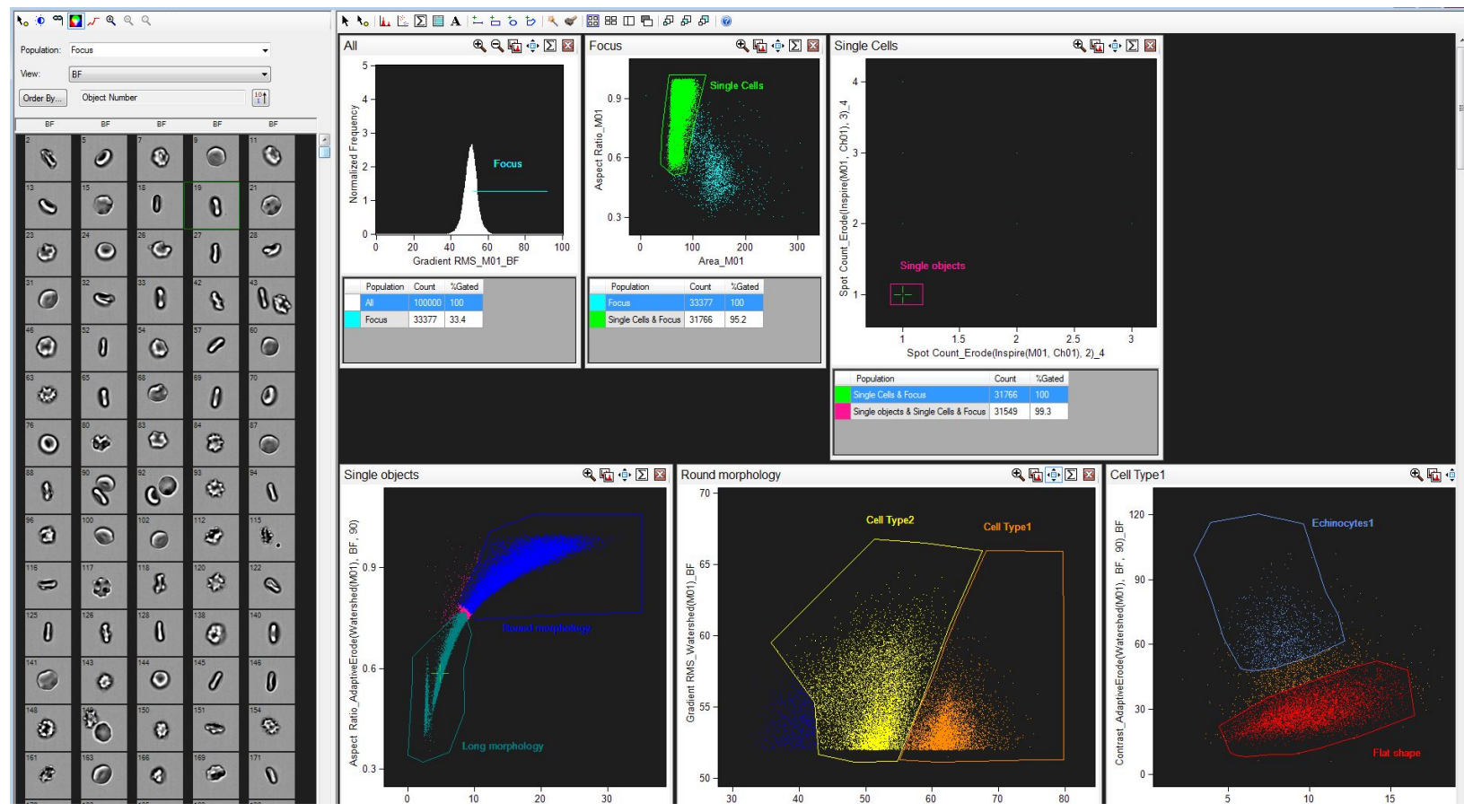

Figure 2-8: Layout of a .daf file created by a template file geared for the analysis of RBCs. Typical layouts include the image gallery (left) and the analysis area (right) that generates graphs and associated statistics. 


\subsubsection{Using Masks, Functions and Features in IDEAS ${ }^{\circledR}$}

The IDEAS ${ }^{\circledR}$ software application provides a large selection of masks, functions and features for image analysis [182]. Firstly, a default mask algorithm is applied to each image in each channel during the formation of the .cif file, and is labelled as $M \#$, where \# is the channel number [181]. Two Boolean default masks, combined and not combined, represent the union and complement of all channel mask ROIs and are also included with default images.

When the .cif file is opened with the default template, the resulting .daf file displayed in IDEAS ${ }^{\circledR}$ provides all images with the default masks applied to them. These default masks cannot be replaced, however, specialized functions can be used to make adjustments to any default mask. Furthermore, Boolean logic commands (And, Not, Or) can be applied to combine or eliminate different default masks or their applied functions [182]. A list and description of all functions available in the mask manager is provided in the Appendices, Table A-4. Figure 2-9 displays an example of the user interface used to merge multiple default masks or functions to create a custom mask that will be later saved for application on any image [182].

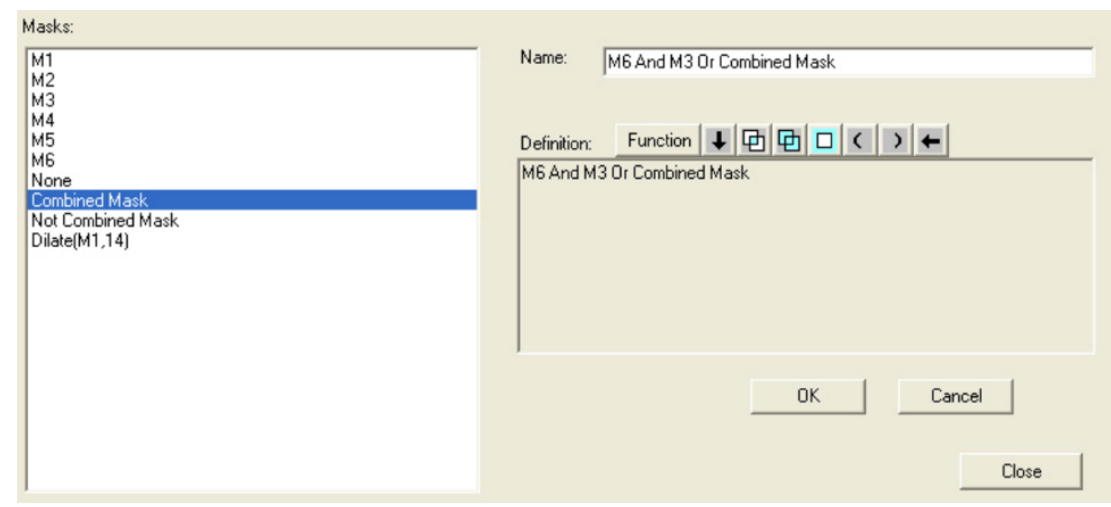

Figure 2-9: Example of the user interface for creating a new, custom mask on the IDEAS ${ }^{\circledR}$ mask manager; all default masks and created functions are available for manipulation with Boolean commands. The mask is saved and can be applied on any image. Adapted from [182].

Various features can be applied to the image ROIs defined by the applied masks. A feature is a mathematical expression of the image's quantitative or spatial information [182]. Values that are generated by each feature for each image can be plotted in a figure, and can be statistically analyzed for an image population. Any quantity and selection of features can be applied to the 
masked images. Over 60 features under specific categories are available on the feature manager [182]. A brief description of each feature category and a list of their associated features is presented in the Appendices, Table A-5. Figure 2-10 displays an example of the user interface for selecting all associated features of a category called Texture using the IDEAS $^{\circledR}$ feature manager. In this example, these features under Texture are applied to all cell images in channel 4; mask M4 is applied to all cells [182].

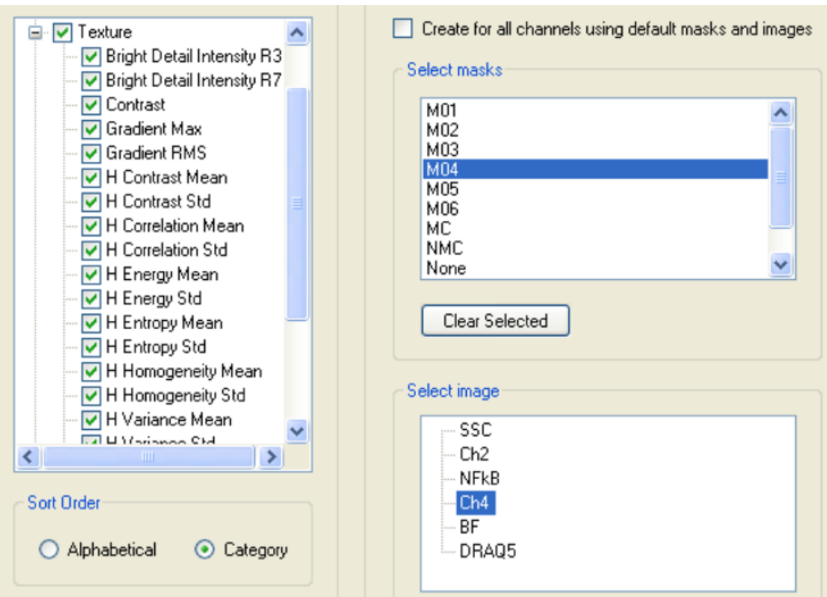

Figure 2-10: Example of selecting multiple features for the analysis of channel 4 images; mask M4 was chosen for ROI detection. Adapted from [182].

\subsubsection{Graph/Statistics Generation in $\operatorname{IDEAS}^{\circledR}$}

As the .daf file is updated with customized masks and features, graphs can be generated to provide visual guidance on the discriminatory capabilities. Graphs can be generated with one feature to create frequency distributions (histograms), or two variables to generate scatter plots [182]. ROIs can be manually traced over regions within the plot to create subpopulations. Clicking on the data points within a histogram/scatter plot highlights its corresponding image; this tool allows for visual verification during ROI selection and enhances the image segmentation accuracy. Once these plots are displayed on the analysis area of the daf file, statistical measures such as mean, mode, standard deviation and variance can be acquired for each ROI. In relation to subpopulations, statistical parameters such as count, percent total and 
percent gated are available. Figure 2-11 displays an example of selecting statistical measures to evaluate a feature on the sample population [182].

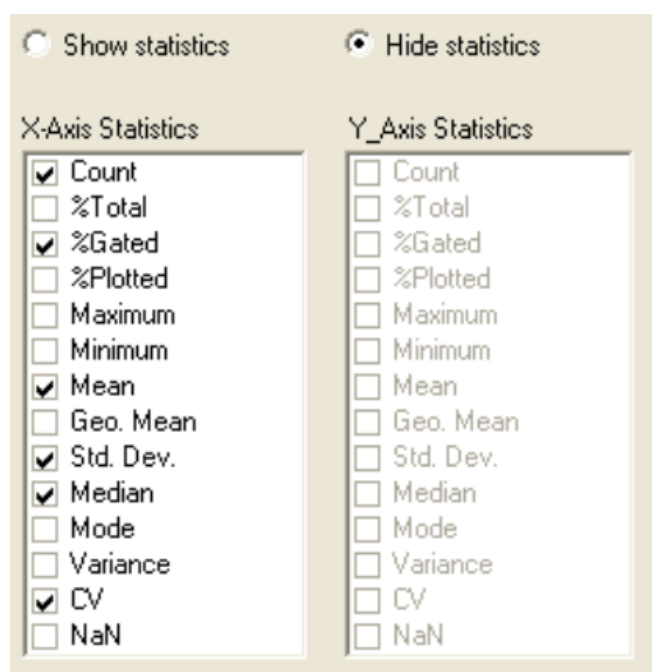

Figure 2-11: Example of selecting multiple parameters in the statistics area. Adapted from [182].

\subsubsection{Image Segmentation in IDEAS ${ }^{\circledR}$ : feature finder, truth populations and ROI tracing}

IDEAS $^{\circledR}$ provides a proprietary tool that automatically finds the best features to more accurately gate subpopulations. The feature finder can be used to calculate the features that best discriminate between images of two subpopulations. A set of hand-picked images of each subpopulation, known as "truth populations", need to be used for the feature finder to obtain the best discriminators for those subpopulations. These features are then plotted against each other for the sample population,

Subpopulations are gated based on traced regions of interest (ROIs). To aid in gaiting, truth populations can be overlaid on the sample population visually estimate placement of ROIs. Furthermore, since each dot on a plot is addressable to an image, subpopulation gates based can be visually verified to refine location of ROIs (see Section 2.4.3). Examples of gaits using ROI tracing can be seen in Figure 2-8. 


\subsection{VisualSonics Vevo LAZR ${ }^{\circledR}$ PA Device}

VisualSonics, a Canadian company founded in 1999, produced the first commercial high frequency, array based ultrasound imaging systems [183]. Over the years, their Vevo product platform has become a gold standard in preclinical in vivo imaging [184]. By 2011 the company released a photoacoustic system, Vevo LAZR (LAZR for short), combining optical sources with their existing preclinical ultrasound imaging system (Vevo 2100). The Vevo LAZR imaging platform consists of real time co-registered PA and US images, with 3-D and Doppler imaging capabilities [183].

\subsubsection{Vevo LAZR ${ }^{\circledR}$ Platform}

Figure 2-12 displays the four major components of the LAZR platform. A LAZR cart that houses the laser optical system feeds laser light through a fiber optic probe that is integrated with a linear array ultrasound transducer (termed LZ series). the PA measurements take place in a LAZRTight steel cabinet to block any exposure to laser light. The signals are sent to the Vevo 2100 US cart that houses the controls and displays PA and US images in real time [185].

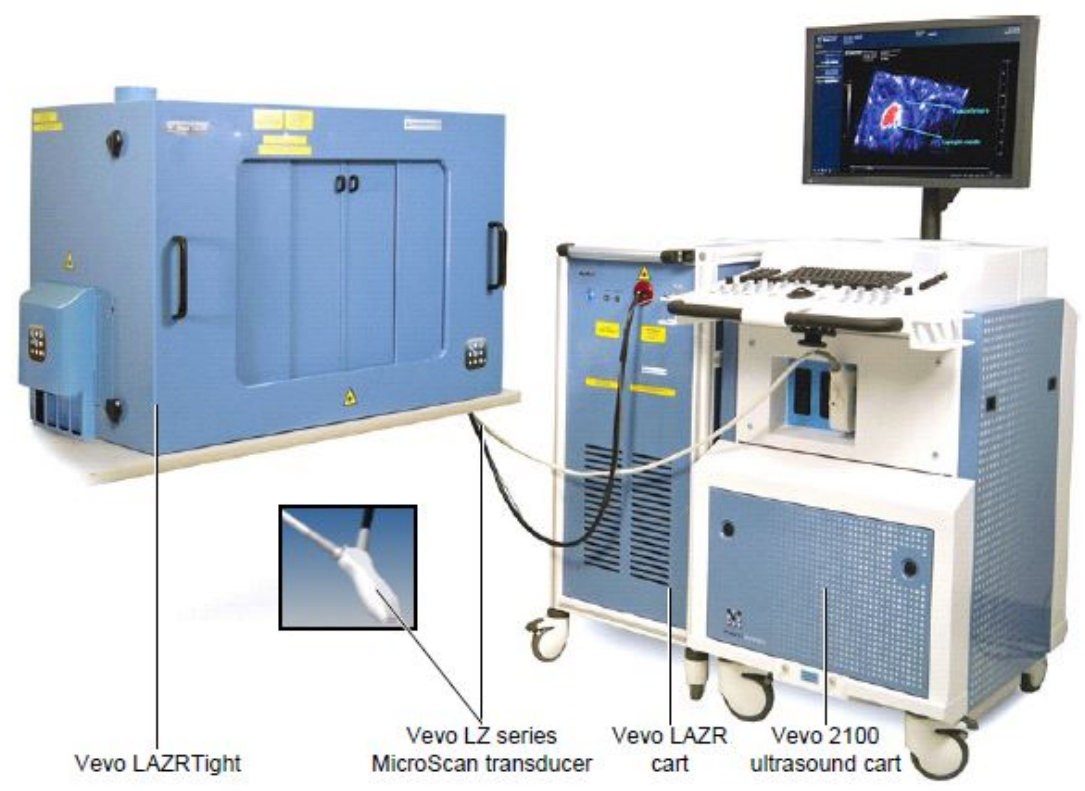

Figure 2-12: The Vevo LAZR Photoacoustic imaging system and the major system components. Adapted from [185]. 


\subsubsection{LZ Fiber-Optic Linear Array Transducers}

Two models of the LZ fiber-optic linear array transducers, LZ250 and LZ550, accompany the LAZR system. Table 2-2 summarizes their broadband frequency ranges, axial and lateral resolutions [186]. The center frequency for LZ250 and LZ550 is $21 \mathrm{MHz}$ and $40 \mathrm{MHz}$ respectively [187]. Both transducers consist of an array of 256 piezoelectric elements and is encased in a housing that integrates the ultrasound coaxial cable and optical fiber bundles into one package [188]. Figure 2-13 provides an illustration of the LZ probe and the arrangement of optical and acoustic sources at the tip of the transducer [189].

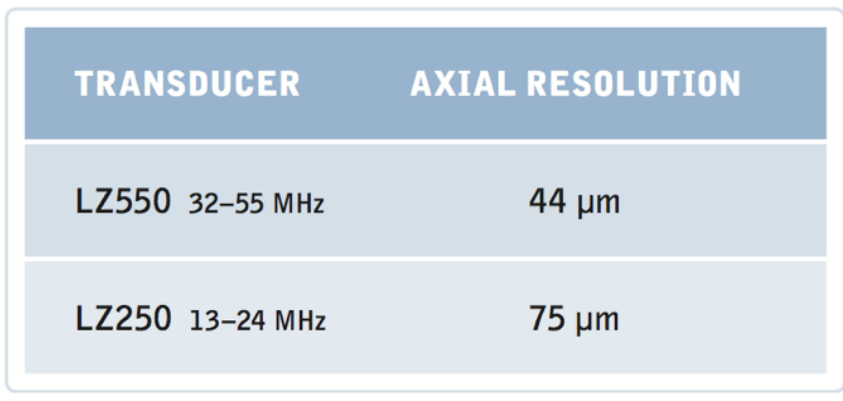

Table 2-2: Broadband frequency range axial and lateral resolutions for two LZ ultrasound transducer models [186].

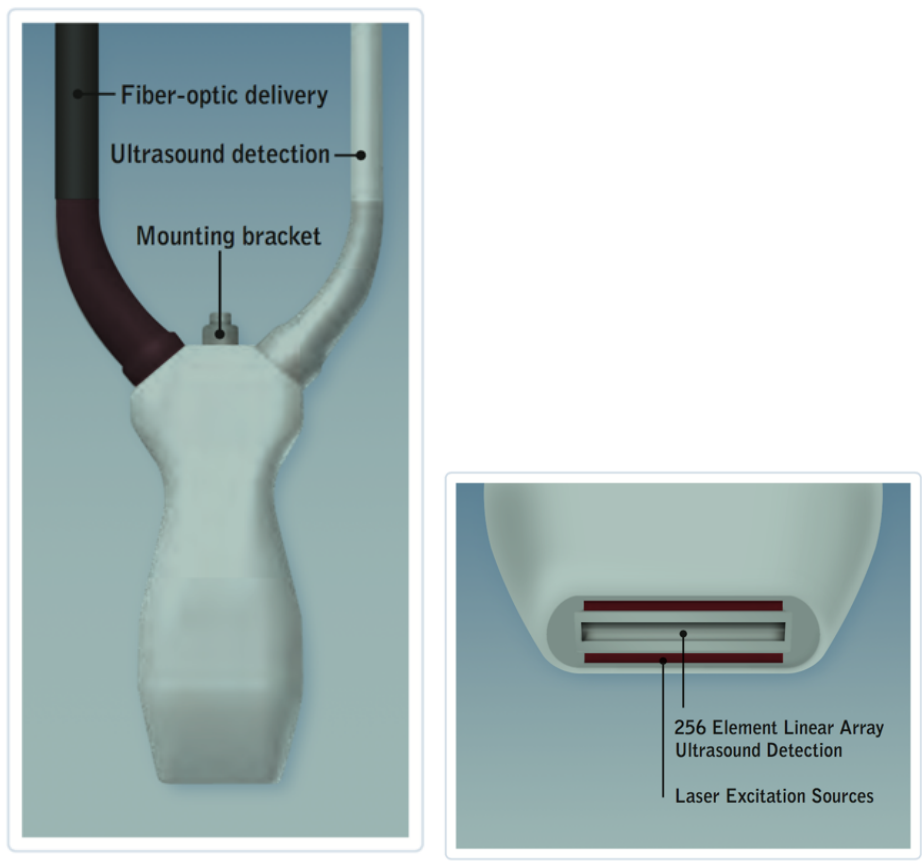

Figure 2-13: (left) Illustration of LZ ultrasound transducer encasement and (right) orientation of optic and acoustic sources at tip of the transducer. Adapted from [188]. 
In the LAZR cart, monochromatic light between $680-970 \mathrm{~nm}$ is produced by a tunable flashlamp pumped Q-switched Nd:YAG laser that contains an optical parametric oscillator (OPO) and a second harmonic generator [189]. The illumination source has a repetition frequency of $20 \mathrm{~Hz}$, pulse width (duration) of 4-6ns, pulse energy of approximately $30 \mathrm{~mJ}$ and a spot size of $24 \mathrm{~mm}^{2}$ $(1 \mathrm{~mm} \times 24 \mathrm{~mm})$ [188]. Figure 2-14 displays the geometry of the optical and ultrasound beam, where it is observed that the rectangular optic bundles are oriented $30^{\circ}$ relative to the imaging plane [189].
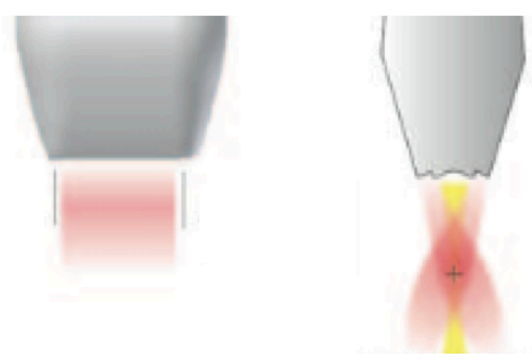

Figure 2-14: (left) Illustration of a lateral view of the LZ light source geometry and (right) axial view of the optical (red) and ultrasound (yellow) beam geometry. Adapted from [188].

\subsubsection{OxyHemo Tool for $\mathrm{SO}_{2}$ Analysis}

The LAZR system software interface allows the user to tune wavelengths within the 680-970nm range for multispectral imaging [185]. Furthermore, a proprietary oxygenation mapping mode called OxyHemo sequentially acquires photoacoustic signals at $750 \mathrm{~nm}$ and $850 \mathrm{~nm}$ and replaces the PA image with an $\mathrm{SO}_{2}$ map [188]. The software settings allow for an ROI to be traced within an image and calculates the average $\mathrm{SO}_{2}$ within that ROI. An example of this feature is described in Figure 2-15. 


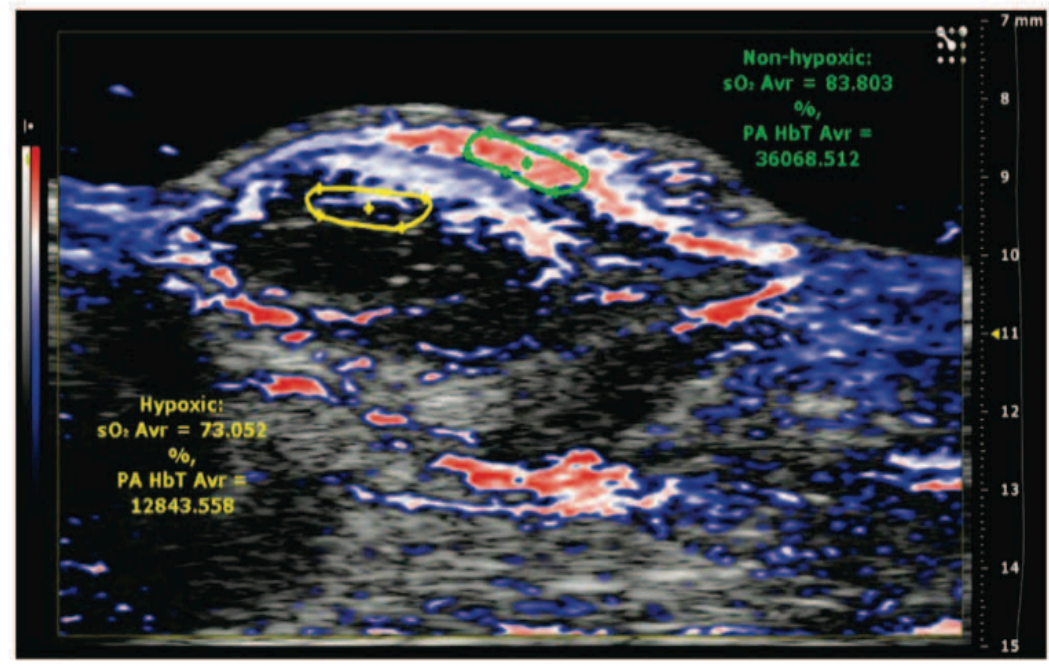

Figure 2-15: Vevo LAZR PA $\mathrm{SO}_{2}$ map overlaid on a coregistered B-mode US image of a Lewis lung carcinoma in a mouse flank. Green/yellow ROIs manually picked; $\mathrm{sO}_{2} \mathrm{Avr}$ represents the average percentage $\mathrm{SO}_{2}$ value calculated for the ROI. Adapted from [188].

\subsection{Control Experiments}

\subsubsection{Investigation of Potential PA Signal from Blood Bag Cover}

A controlled experiment was devised to investigate if the blood bag plastic cover (Section 1.3.3) contributes its own PA signal. An open cylindrical glass container of approximately $2.5 \mathrm{~cm}$ diameter and $15 \mathrm{ml}$ volume was covered with a piece of the blood bag plastic (Figure 2-16). Holes were bored through sides of the cylinder to fill the covered cylinder with water or RCC. In separate measurements, water and RCC were inserted into the cylinder and the ends were sealed with Vaseline. Images of the cylinder and setup are displayed in Figure 2-16.

Within the LAZRTight (Section 2.5.1), the cylinder was immersed into a water tank, and was held underwater using blocks resting on the cylinder. The LZ250 transducer (Section 2.5.2) was placed $10 \mathrm{~mm}$ above the plastic cover of the cylinder. PA measurements were taken at $750 \mathrm{~nm}$ under PA mode (further details on the LAZR setup, calibration and mode controls are provided in Section 2.7.4). For each measurement, 10 image frames were acquired. All raw data from the frames were uploaded onto MATLAB ${ }^{\circledR} 2016$ (Mathworks Inc., Natick, MA, USA) scripts for the purpose of beamforming. 


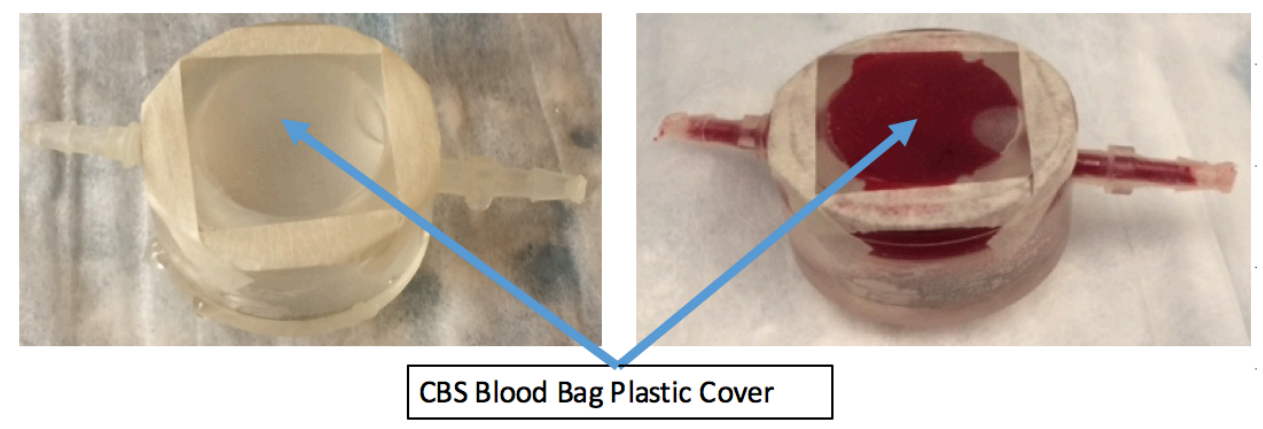

Figure 2-16: A cylindrical glass container, covered with blood bag plastic and filled with (left) water and (right) blood; the extensions used to inject the medium were sealed with vaseline.

\subsubsection{Agreement between LAZR and FLEX $\mathrm{SO}_{2}$}

A control experiment was performed to verify the compatibility between LAZR and FLEX measurements of $\mathrm{SO}_{2}$. A sample of human lyophilized hemoglobin (Section 2.1.1) was diluted at a concentration of $40 \mathrm{mg} / \mathrm{ml}$ in PBS, and mixed thoroughly using a vortex at $1800 \mathrm{rpm}$. A solution of sodium dithionite (hydrosulfite) was prepared with PBS at a concentration of $20 \mathrm{mg} / \mathrm{ml}$. The sodium dithionite was used to reduce the predominantly metHb solution into deoxyHb [190,191]. Both solutions were mixed at a 1:1 ratio, creating the recommended hemoglobin dilution concentration of $20 \mathrm{mg} / \mathrm{ml}$ [170].

The heavily deoxygenated hemoglobin solution was oxygenated by aerating the sample in pair of syringes connected with a nozzle. The level of oxygenation was proportional to the amount of time spent aerating the solution. Using this aeration technique, a 1-minute aerated solution and a 5-minute aerated solution were produced.

The three solutions of deoxygenated hemoglobin, briefly oxygenated hemoglobin ( 1 minute) and heavily oxygenated hemoglobin (5 minutes) were measured using the same setup as described in Section 2.6.1. PA measurements were carried out on the Oxy-Hemo mode, acquiring $\mathrm{SO}_{2}$ values from 10 frames (further details of acquisition in Section 2.7.4). Simultaneously, three FLEX measurements were made for each solution. 


\subsection{RBC FLEX/IS ${ }^{\mathrm{x}} /$ LAZR Experimental Protocol}

To address both hypotheses (Section 1.11), a protocol was developed to include FLEX (Section 2.2), IS ${ }^{X}$ (Section 2.3) and LAZR (Section 2.5) measurements to monitor stored RBCs on the same day using all instruments. The measurements were repeated every 3-5 days until the expiration date of the RCC blood bags. Implementation of the entire protocol on seven blood bags took approximately 5-6 hours. Due to the frequency and duration of the experimental protocol, care was taken to reduce the exposure of blood samples and blood bags to nonrefrigerated environments.

\subsubsection{RCC Sample Extraction}

RCC blood bags were taken out of the storage location and placed in a biosafety cabinet. The bags were lightly massaged to homogenize the RCC within the bag. A 20 gauge needle was attached to a safePICO syringe and inserted into the sampling site coupler (Section 2.2.1); a picture displaying this process in shown in Figure A-1 in the Appendices. Approximately $1 \mathrm{ml}$ of sample was extracted and discarded to remove any residual blood left within the coupler and to access RCC within the blood bag. Another $1 \mathrm{ml}$ sample was collected into the syringe, after which the needle was discarded and the safeTIPCAP (Section 2.2.1) was secured immediately (Figure A-2). This extraction procedure was repeated with two more syringes, totaling three samples per bag. After extraction, the blood bags were placed back into the refrigerator and the samples were placed in a plastic pouch to transport to the FLEX (Figure A-3).

\subsubsection{FLEX Measurement}

Samples were place in the designated slots, three syringes at a time, for automated mixing, aspirating and $\mathrm{SO}_{2}$ measurement (Figure A-4). Upon acquisition of data, the syringes were immediately placed back into the plastic pouch, covered in bubble wrap and stored in a cooled styrofoam box for transportation to the IS ${ }^{\mathrm{X}}$. 


\subsubsection{IS ${ }^{\mathrm{X}}$ Measurement}

At the facility housing the IS ${ }^{X}$, syringe samples were transferred from the styrofoam box to the fridge where they were extracted one at a time for measurements.

\section{Experimental Protocol}

For each sample, an aliquot of $5 \mu \mathrm{l}$ was diluted with $200 \mu \mathrm{l}$ of PBS in a vial and was placed into the IS $^{\mathrm{X}}$ sample holder for automated aspiration and image acquisition. 100,000 images were captured under $60 \times$ image magnification. After the experiment, the syringes were discarded as

per CBS guidelines. A picture of the IS ${ }^{\mathrm{X}}$ device and location of the sample holder is shown in Figure A-5.

\section{Image Analysis}

All raw data files (each file pertaining to one measured sample) were transferred via USB to a portable hard drive to be uploaded onto a computer with a Windows 764 bit OS, consisting of an Intel Core i7-4790 processor and a 16 GB memory. Each raw data file was opened on the IDEAS $^{\circledR}$ software platform and was processed automatically by the developed RBC morphology image segmentation template (see Section 3.2). The processed file provided the spheroechinocyte population percentage.

\subsubsection{LAZR Measurement}

Both LZ transducer probes (Table 2-2) were used for the PA measurements. The intention for including both probes was twofold. Firstly, it allowed for an investigation to which transducer, if any, was more accurate in determining $\mathrm{SO}_{2}$ values of blood bags in situ. Secondly and more significantly, it expanded the band width data $(13-55 \mathrm{MHz})$ for radiofrequency spectroscopy analysis (Section 1.5.3). LAZR measurements followed shortly after the $\mathrm{IS}^{\mathrm{X}}$ experiments (Section 2.7.3).

\section{$\underline{\text { US/PA Setup }}$}

US images were used as a verification tool for the location of the blood bag plastic cover, which produced a strong signal due to high impedance mismatch with respect to water. The PA images 
were overlaid on co-registered US images during $\mathrm{SO}_{2}$ acquisition. Table 2-3 outlines other parameters that were set with the user interface for US and PA images.

\begin{tabular}{|c|c|c|}
\hline PARAMETER & ULTRASOUND & PHOTOACOUSTICS \\
\hline MODE & B-Mode & PA/Oxy-Hemo \\
\hline GAIN & 50 & 70 (Maximum) \\
\hline FRAMES STORED & 10 & 10 \\
\hline IMAGE DEPTH & $17 \mathrm{~mm}($ LZ250) & $17 \mathrm{~mm}$ (LZ250) \\
& $15 \mathrm{~mm}$ (LZ550) & $15 \mathrm{~mm}$ (LZ550) \\
\hline FOCAL REGION & Variable & Fixed (11mm) \\
\hline
\end{tabular}

Table 2-3: List of parameters set for US and PA image generation on the LAZR.

\section{Optical Source Calibration}

As the monochromatic light beams exit the LAZR cart (2.3.1) a small fraction of the beam is deflected for energy determination. Before measurements, this beam energy mechanism was calibrated to the laser energy output at the tip of the transducer probe. A laser energy sensor (OPHIR, Utah, USA) was positioned just underneath the probe, and was connected directly to the LAZR cart. The calibration mode was run, initiating an internal calibration. An image of the setup for this calibration step is shown in Figure A-6 in the Appendices.

\section{Blood Bag LAZR Container}

For PA measurements, both the blood bag and transducer had to be immersed in a water bath to reduce acoustic impedance mismatches of the propagating PA signal. Underwater, the blood bags needed to lie flat and stationary for optimal signal output; furthermore, internal air pockets within the bags needed to be removed from the region of illumination to reduce signal artifacts. To address these requirements, a custom blood bag LAZR container was designed.

A schematic representation of the blood bag LAZR container is shown in Figure 2-17. Figure A7 in the Appendices displays the positioning of the blood bag LAZR container within the LAZRTight (Section 2.3.1). The rectangular plexiglass tank $(25 \times 14 \times 12 \mathrm{~cm})$ was filled with cold water in order to minimize warming of the blood bags. Handling procedures were developed 

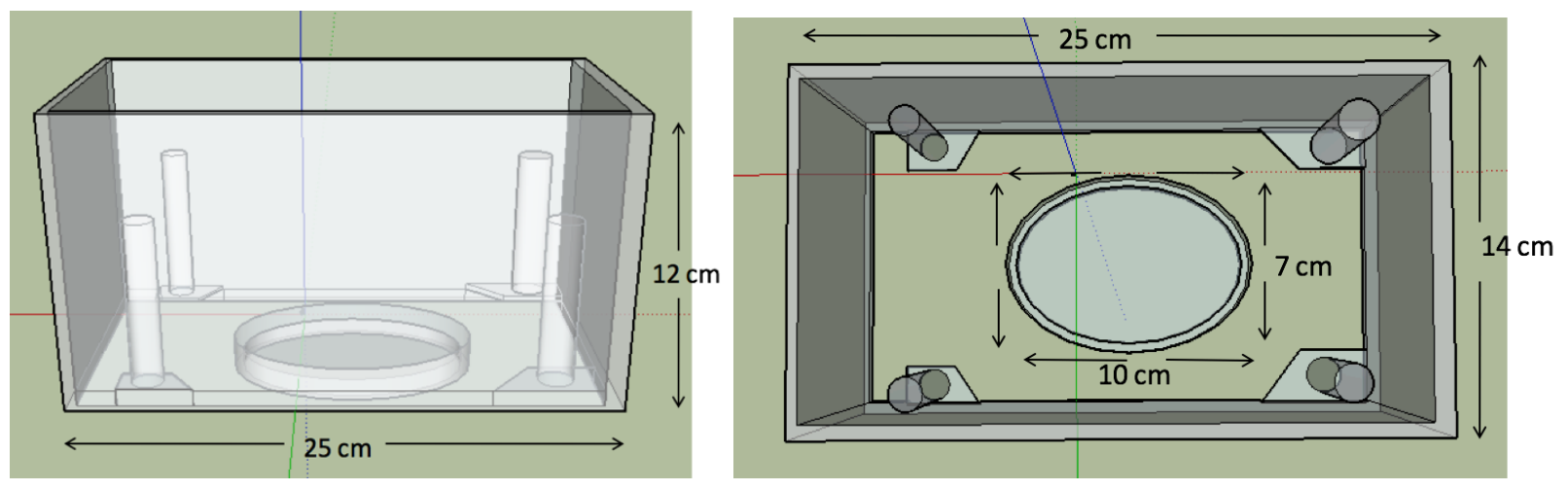

Figure 2-17: (left) Side view and (right) Ariel view of a schematic representation of the blood bag LAZR container. Illustration courtesy of K. Bagga.

when immersing the blood bags to keep blood bag air bubbles from occupying the illuminating region. The procedure is illustrated in a series of images in Figure A-8 in the Appendices. The bags were held flat and stationary using a plexiglass lid, containing a central elliptical hole (major:minor axis 10:Cm) allowing for the Vevo LAZR probe to scan the bag.

\section{Measurement of $\mathrm{SO}_{2}$}

The transducer was immersed in the container, under water, and was kept approximately $10.5 \mathrm{~mm}$ above the boundary of the blood bag, so that the optical focal zone $(11 \mathrm{~mm})$ was located under the plastic boundary of the bag allowing for maximum energy deposition in the RCC. Accurate positioning was achieved using feedback from the real-time US images. In the process of submerging the transducer, an air pocket was often trapped in a crevice enclosing the linear array (Figure 2-13), and its presence was easily detected from the high level of scattering displayed on the US images. The air bubble was removed using a finger, with its removal was verified with real-time B-mode images (left image of Figure A-9, Appendices).

The right image of Figure A-9 displays the setup of the transducer probe over a blood bag within the boundaries of the lid hole. The LAZRTight was closed and images of the blood bag in PA mode were generated. Once all parameters were verified, a measurement in Oxy-Hemo mode was carried out. The Oxy-Hemo mode used laser wavelengths at 750 and $850 \mathrm{~nm}$, and used signals from both wavelengths to create an $\mathrm{SO}_{2}$ map (Section 2.5.3). After 10 image frames were collected, an ROI was traced over the central, homogenous part of the $\mathrm{SO}_{2}$ map of the first 
frame. An average of the $\mathrm{SO}_{2}$ values of all pixels within the selected region was produced. The image ROI was applied to the other 9 frames, producing $10 \mathrm{SO}_{2}$ values for that particular measurement. This procedure was repeated for two other locations on the RCC blood bag, accumulating (in total) $30 \mathrm{SO}_{2}$ values per RCC blood bag per PA measurement. An image of the PA setup and $\mathrm{SO}_{2}$ map is displayed in Figure 2-18.

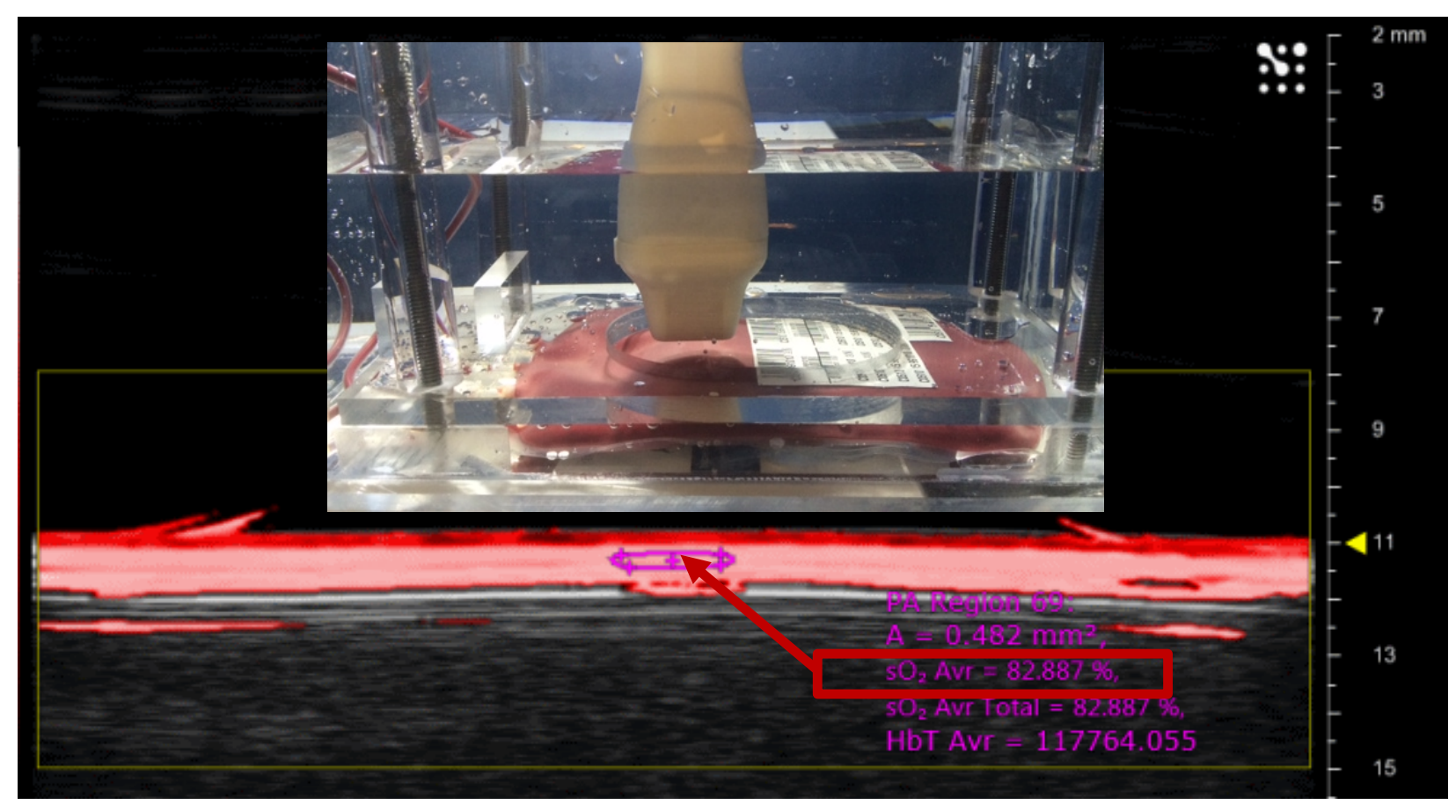

Figure 2-18: $\mathrm{PA} \mathrm{SO}_{2}$ map of a blood bag, including the traced region (purple circle) for $\mathrm{SO}_{2}$ calculation (red box). For increasing $\mathrm{SO}_{2}$, scale ranges from dark red to light pink. Background black/white signal represents co-registered US image. Yellow arrow represents the optical focal depth $(11 \mathrm{~mm})$. Inset displays setup used for measurement.

\subsection{Data Handling}

All raw data was uploaded onto excel for organization and basic statistical processing (mean, standard deviation). All statistically processed data was uploaded onto MATLAB 2016 (Mathworks Inc., Natick, MA, USA) for the plotting. Bland-Altman plots were used to investigate the level of agreement between the values of one parameter measured using two different techniques [192]. 


\section{Chapter 3 : Results}

\subsection{Control Experiment Results}

Figure 3-1 plots the absolute value of the Hilbert transform of the average PA radio-frequency (RF) signal as a function of depth, acquired from a transducer placed above a glass cylinder containing either water (blue) or approximately $85 \%$ oxygenated RCC (red); during both instances, the cylinder was covered with $0.35 \mathrm{~mm}$ thick blood bag plastic (Section 2.6.1). The average RF signal was calculated from the signals of eleven elements that included five to the left and right of the center element. The transducer was placed $10 \mathrm{~mm}$ above the top boundary of the glass cylinder cover.

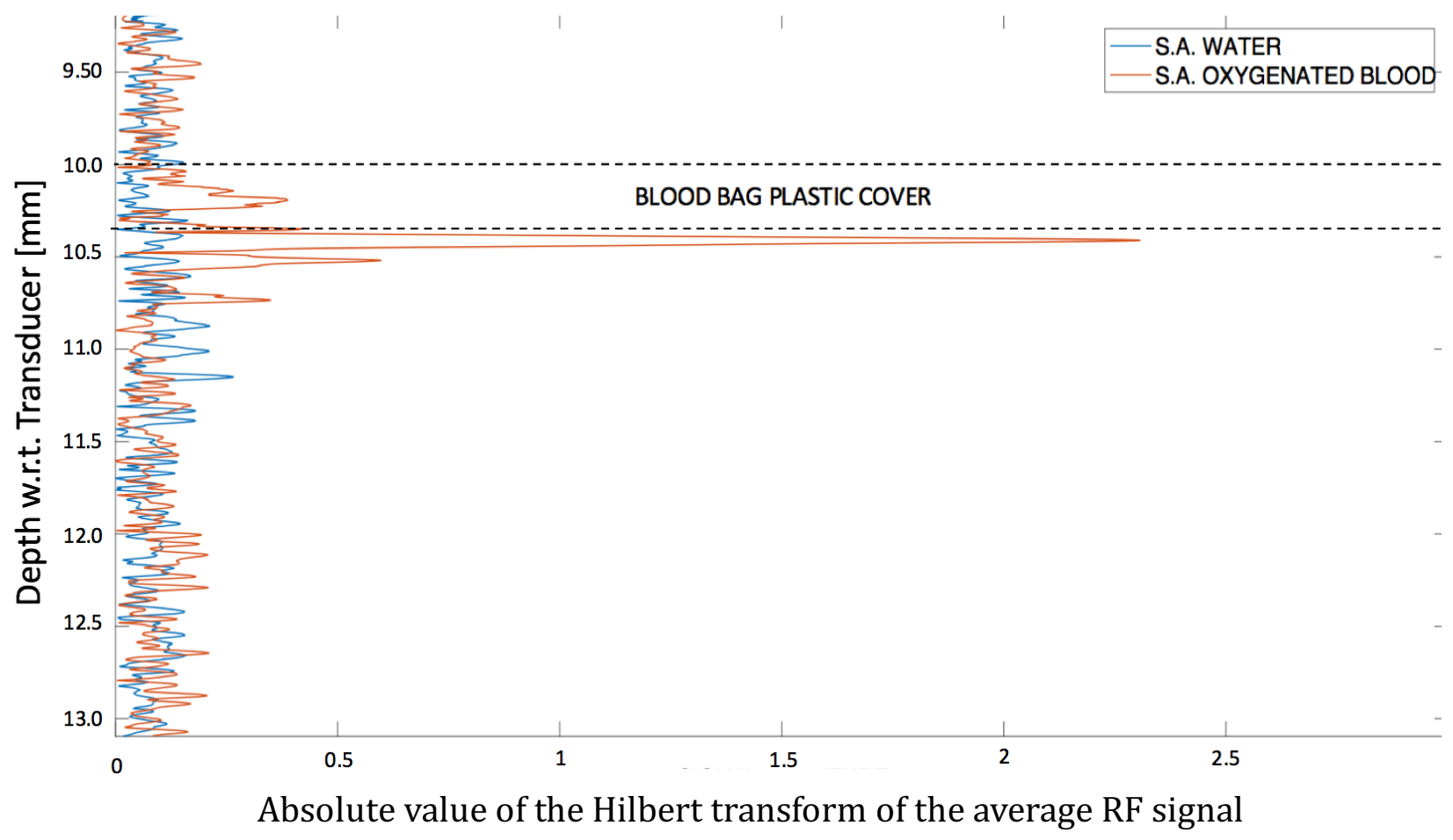

Figure 3-1: Absolute value of the Hilbert transform of the average RF signal (11 lines) of water (blue) and oxygenated ( $~ 85 \%)$ RCC (red) in a glass container covered with $0.35 \mathrm{~mm}$ thick blood bag plastic (dashed lines), whose top boundary is located at $10 \mathrm{~mm}$. 
Figure 3-2 displays the $\mathrm{SO}_{2}$ values acquired from the FLEX (Section 2.2.2) as well as the LZ250 and LZ550 LAZR transducers (Sections 2.5.2, 2.5.3). Three solutions of lyophilized hemoglobin of varying levels of oxygenation were prepared as outlined in Section 2.1.2. For the BGA measurements, the standard deviation bars displayed in Figure 3-2 are of three $\mathrm{SO}_{2}$ values taken from three syringes; for the PA measurements, standard deviation bars are of the 10 frames captured from one measurement.

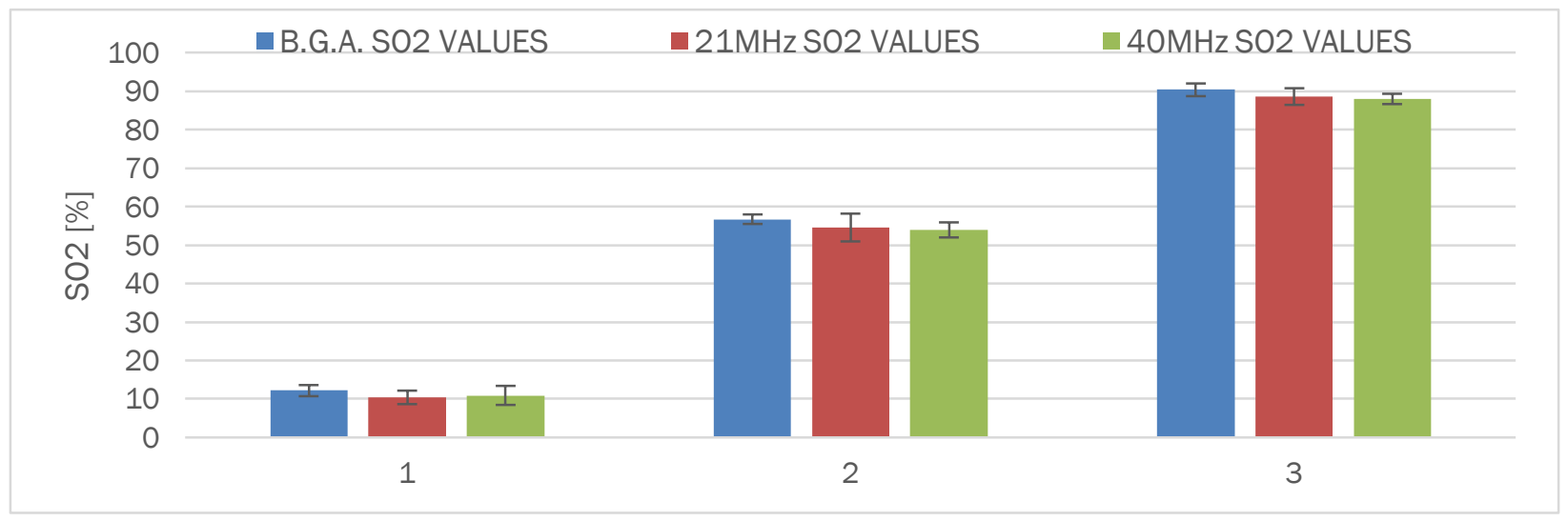

Figure 3-2: FLEX (blue), LAZR LZ250 (red) and LAZR LZ550 (green) mean $\mathrm{SO}_{2}$ values of heavily deoxygenated (1), evenly oxygenated (2) and heavily oxygenated (3) lyophilized hemoglobin. Standard deviation bars represent 3 independent FLEX measurements, and 10 frames of 1 LAZR measurement.

\subsection{RBC Morphology Image Segmentation Template}

The RBC morphology image segmentation template (RBC-MIST) was developed in association with M. Parsons (LTRI, Sinai Health System, Toronto), J. Sebastian (Ryerson University, Toronto) and T. Chang (MilliporeSigma, Seattle, WA). Using typical images collected from samples of RCC, the image segmentation steps making up the RBC-MIST are illustrated in Figures 3-3 and 3-4. Figure 3-3 outlines the gating strategy used to exclude unwanted subpopulations (represented by images A, B and C) from the analysis. The segregation of RBC images into side view and front view subpopulations was carried out in four steps (Figures 3-5 3-8). Figure 3-4 outlines the gating strategy used to segment these side view and front view RBC images into discocyte and spheroechinocyte subpopulations, which was carried out via four steps (Figures 3-9-3-12). 
The plots displayed in Figures 3-9, 3-11 and 3-12 required the use of the IDEAS ${ }^{\circledR}$ feature finder tool to determine the best features to discriminate between the subpopulations; consultation for the collection of RBC truth populations was provided by T. Turner (COI, CBS, Edmonton) (see Section 2.4.4). In an automated format, the number of images collected for each subpopulation (highlighted by curved/right-angled red boxes in Figure 3-4) were considered the total RBC population, from which the relative spheroechinocyte population percentage (right-angled boxes,

Figure 3-4) was determined.

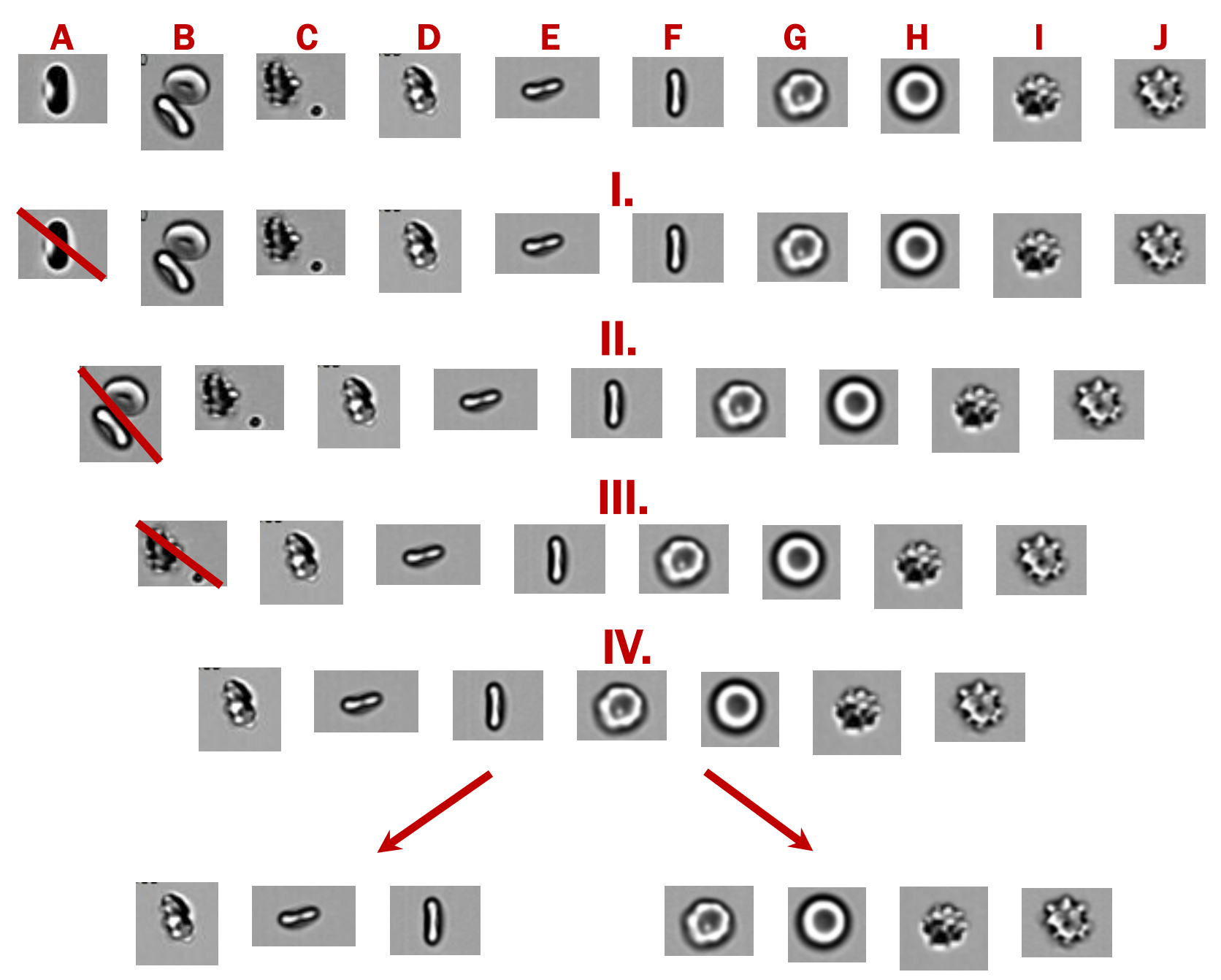

Figure 3-3: (Top Row) Typical RBC images produced by the IS ${ }^{\mathrm{X}}$ IFC system, including unfocused (A), multi-cell (B), cells with calibration beads $(C)$, front view discocytes $(D, E)$, side view discocytes $(F)$, side view spheroechinocytes $(\mathrm{G})$ and front view spheroechinocytes (H, I). (Second Row and Below) Sequential gating based on various mask/feature combinations was used to exclude objects of type A (I.), B (II.), C (III.), and differentiate populations D-F from G-J to classify side view and front view images, respectively. 

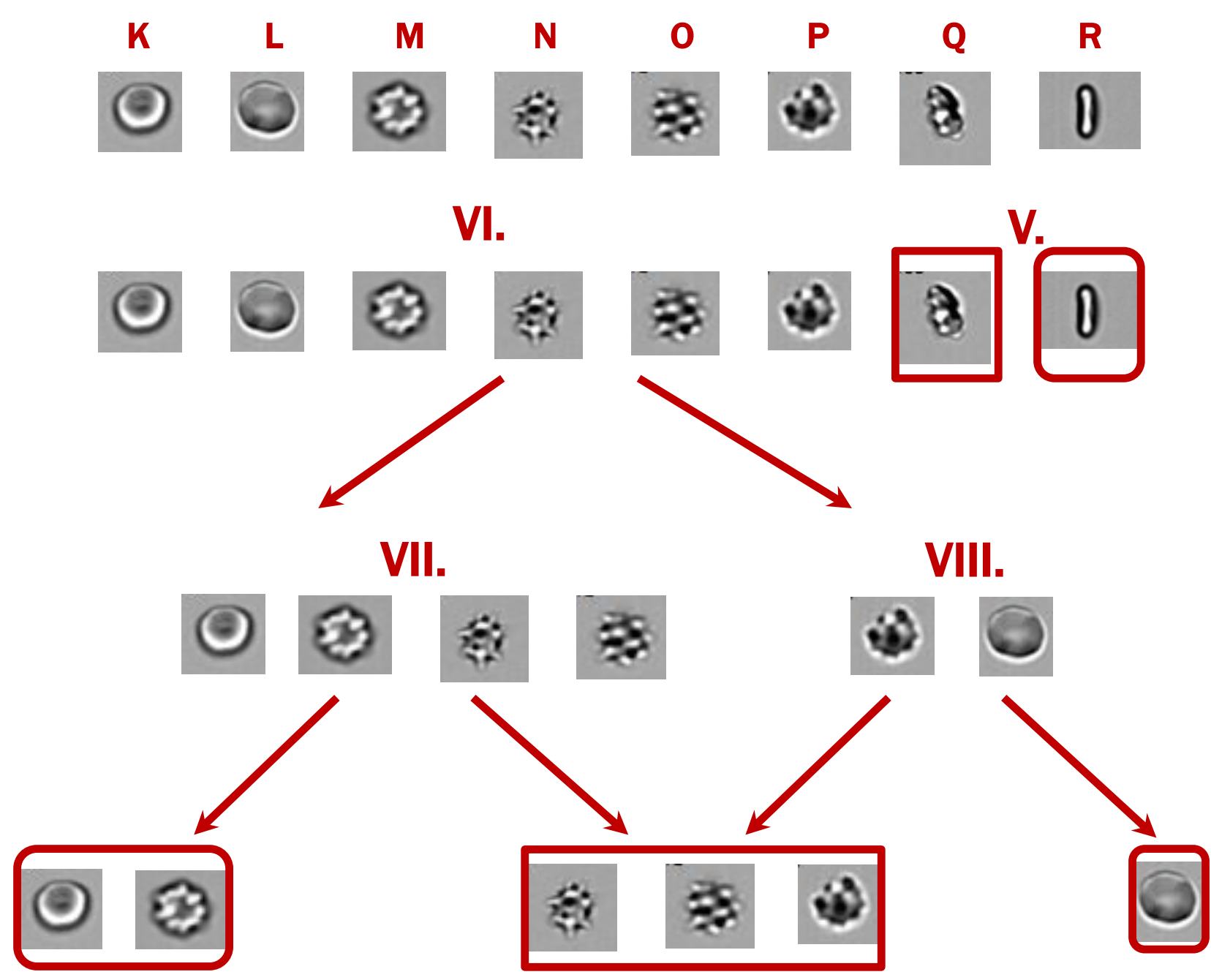

Figure 3-4: (Top Row) Typical RBC images that include smooth/crenated discs (K,L), crenated discoid (M), crenated spheroid $(\mathrm{N})$, smooth/crenated spheres $(\mathrm{O}, \mathrm{P})$, side view spheroechinocytes $(\mathrm{Q})$, and side view discocytes (R). (Second Row and Below) Sequential gating based on various mask/feature combinations (V. - VIII.) was used to differentiate populations $\mathrm{K}, \mathrm{L}, \mathrm{M}$ and $\mathrm{R}$ (curved red box) from N, O, P and Q (right angled red box) to classify discocytes and spheroechinocytes, respectively.

Figure 3-5 shows a histogram used to discriminate between unfocused (blurry) and focused (sharp) images, as illustrated in step I (Figure 3-3). The feature gradient RMS calculated the root mean square (RMS) of the intensity gradient between pixels within the default bright field image mask [182]. By visual verification, images with a gradient $R M S$ value of 54 or below were excluded from further analysis. 
Figure 3-6 displays the plot used to discriminate between single and multi-cell images, as illustrated in step II (Figure 3-3). The aspect ratio feature, calculated as the ratio of the object's minor axis over its major axis, was plotted against the area feature based on the size of the object in square microns [182]. Both features use the default bright field object mask. A gate was created such that object aggregates within the same image field were excluded from further analysis.

Figure 3-7 displays the plot used to discriminate between images containing RBCs with calibration beads and single RBC images, as illustrated in step III (Figure 3-3). Two variations of the erode mask was used to shrink the default mask to cover only the brightest regions of the image [182]. The number of disjointed regions of each erode mask, or spot counts, was plotted against each other. Images with spot count greater than 1 (for either mask) were excluded from subsequent analysis.

Figure 3-8 displays the plot used to discriminate between front view and side view RBC images, as illustrated in step IV (Figure 3-3). Using the watershed mask, the aspect ratio feature was plotted against the circularity feature to discern the object's relative elongation and deviation from a circle, respectively [182]. A gate was created using ROI tracing to segregate side view and front view images for further analysis.

Figure 3-9 provides the plot used to discriminate side view images of spheroechinocytes and discocytes, as illustrated in step V (Figure 3-4). Figure 3-9 has, overlaid on the sample population, the locations of the truth population images for side view spheroechinocytes and discocytes (in purple \& pink respectively). Using the watershed mask, the strongest statistical discriminators (determined from feature finder) were found to be Haralick (H)-variance and symmetry. H-variance is one of many texture features defined by Haralick and colleagues to normalize and characterize the local variation in intensity distribution within the masks [193]. The symmetry feature measured the tendency for an object to have a lobe-like geometry symmetric across an axis [193]. ROIs were traced with the aid of truth populations to gate side view discocyte and side view spheroechinocyte images. 


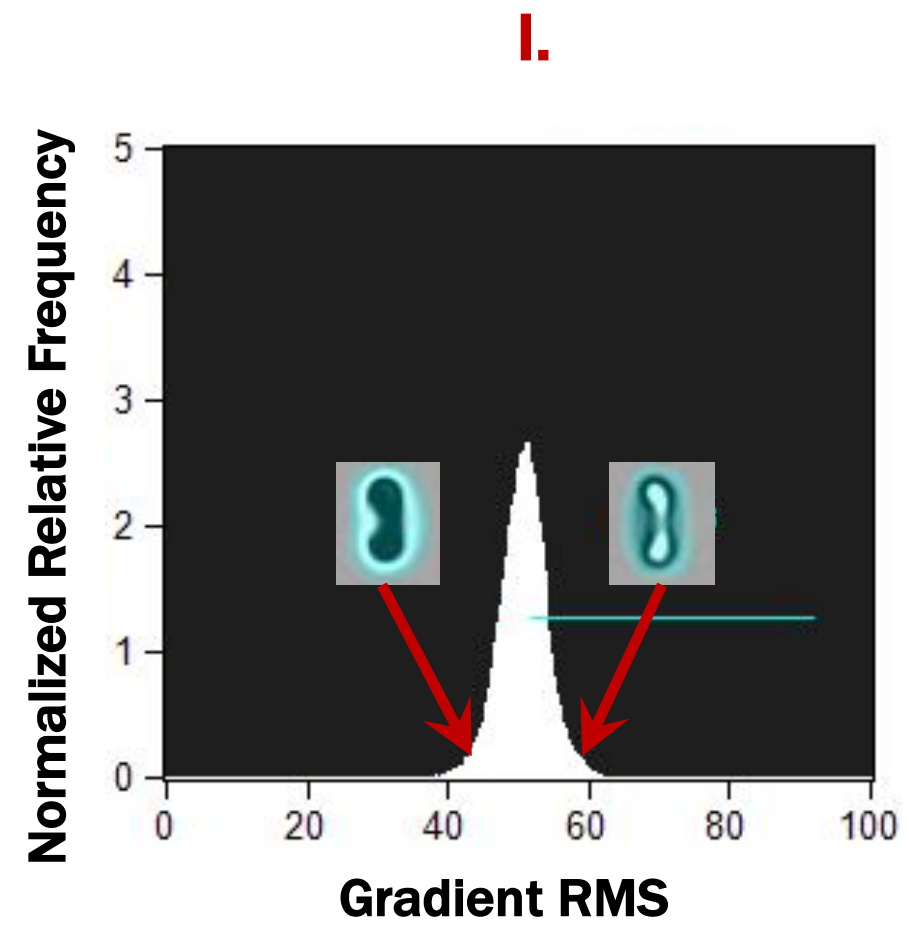

Figure 3-5: (see step I, Figure 3-3); histogram of gradient RMS (counts of normalized relative frequency) within the default bright field mask. Inset images provide examples of unfocused (left) and focused (right) RBC images. Object masks are highlighted in light blue. Value of gradient RMS of images are indicated by red arrows. Images containing gradient RMS values above 54 were selected for further analysis (horizontal light blue line).

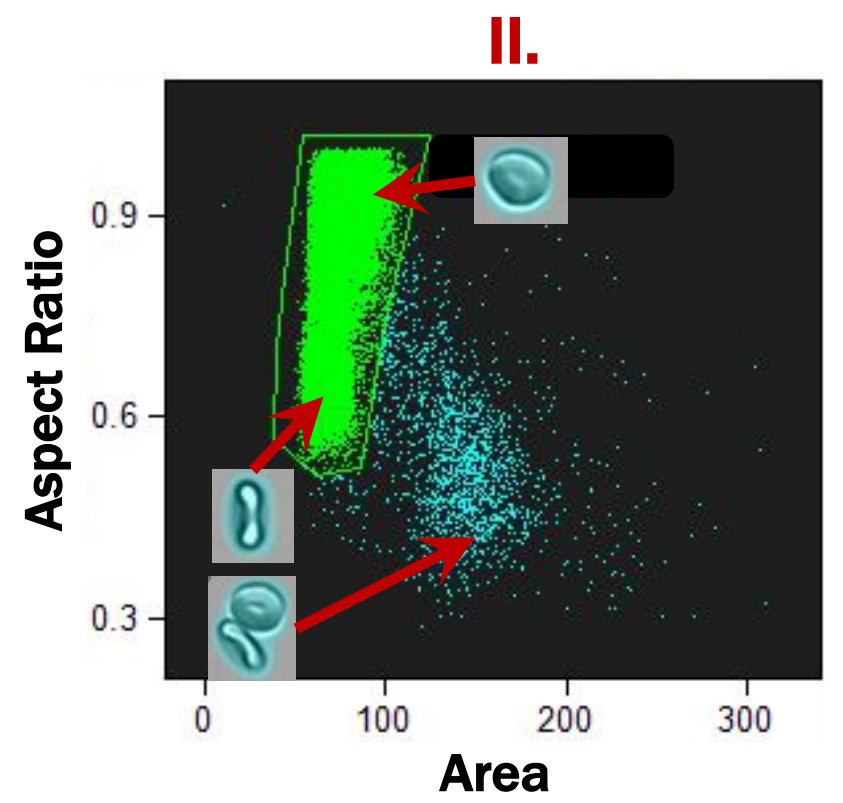

Figure 3-6: (see step II, Figure 3-3); plot of aspect ratio versus area using the default bright field mask. Inset images provide examples of front view (top right), multi-cell (middle right) and side view (bottom left) RBC images. Object masks are highlighted in light blue. Red arrows point to the location of images within the scatter plot. A gate based on images within the ROI (light green boundary) was selected for further analysis. 


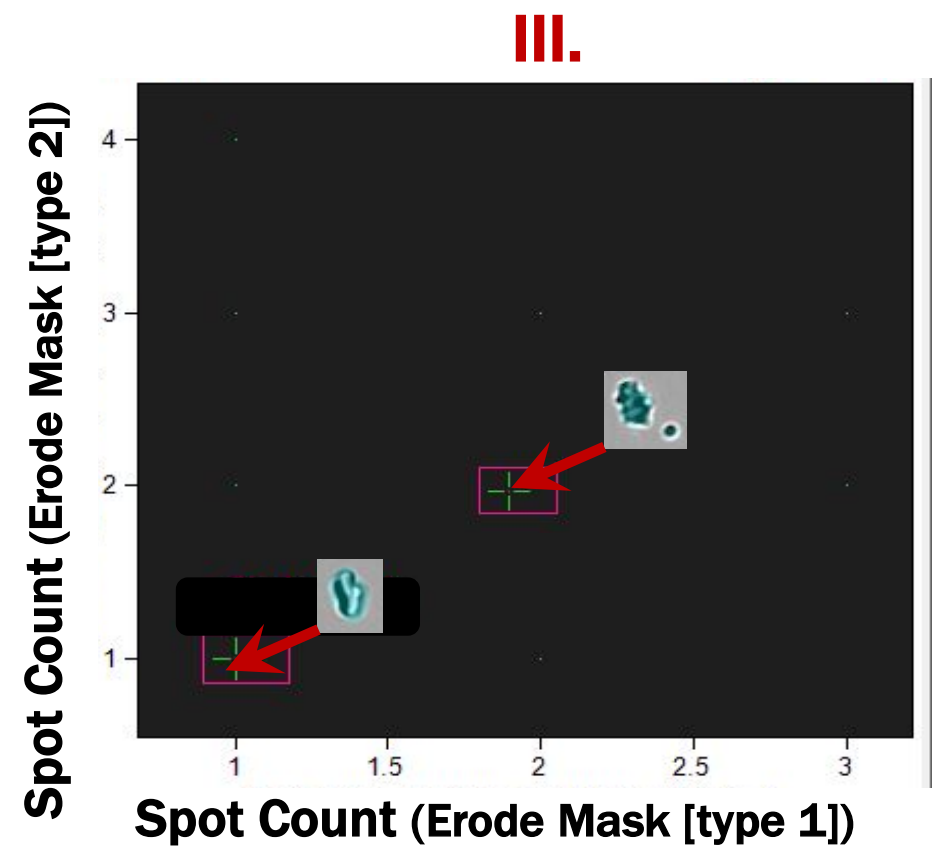

Figure 3-7: (see step III, Figure 3-3); plot of spot count using two variations of the erode mask. Inset images provide examples of cells with calibration beads (top right) and single cell (bottom left) RBC images. Object masks are highlighted in light blue. Red arrows point to the location of images within the scatter plot. Only images with a spot count of 1 (for both masks) were selected for further analysis.

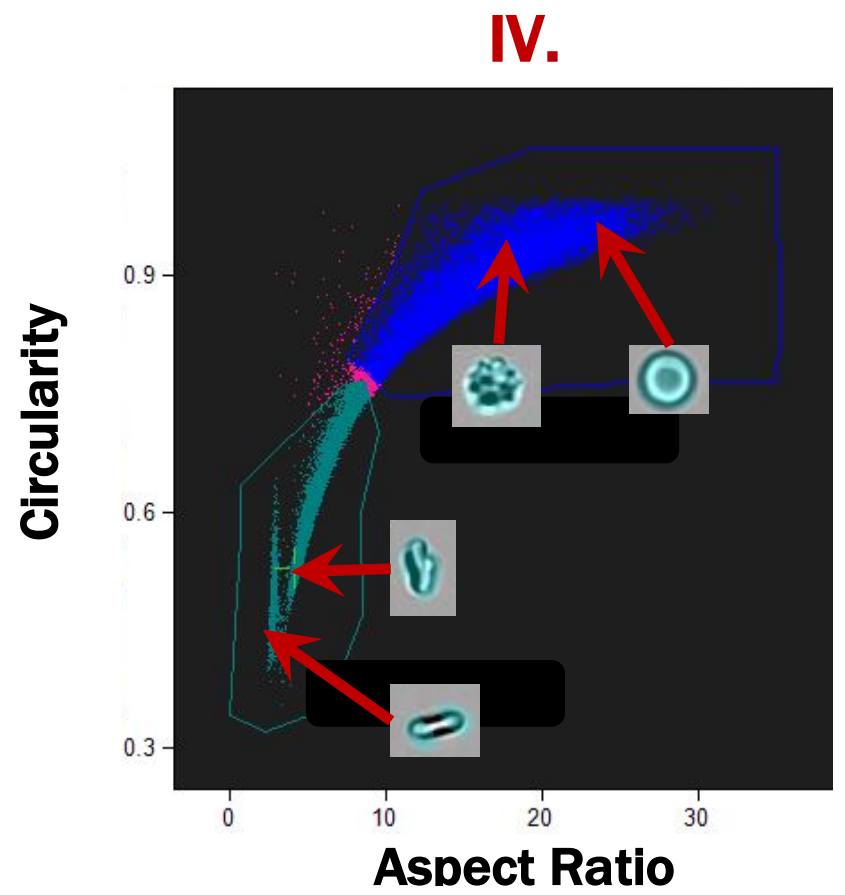

Figure 3-8: (see step IV, Figure 3-3); plot of circularity versus aspect ratio, using the watershed mask with an adaptive erode function. Inset images provide examples of front view (top) and side view (bottom) RBC images. Object masks are highlighted in light blue. Red arrows point to the location of images within the scatter plot. A gate based on images within both ROIs (green/blue boundary) were selected for further analysis. 
Figure 3-10 displays the plot used to discriminate between darker and brighter shaded front view RBC images. Two different masks were applied with the gradient RMS feature: the watershed mask contoured the entire object, while the watershed AND NOT erode mask combination created a contour around the object boundary. Using visual verification, ROIs were traced to gate both subpopulations for analysis.

Figure 3-11 displays the plot used to discriminate between darker shaded spheroechinocytes and discocytes. The feature finder/truth population strategy found contrast and H-variance features to be the best discriminators. The contrast feature measured sharpness in pixel intensity across the image. In the same manner, Figure 3-12 displays the H-correlation mean versus $H$ correlation standard deviation texture feature plot, found to best discriminate light shaded spheroechinocytes from discocytes. For both plots, ROIs were traced with the aid of truth populations to gate subpopulations for analysis.

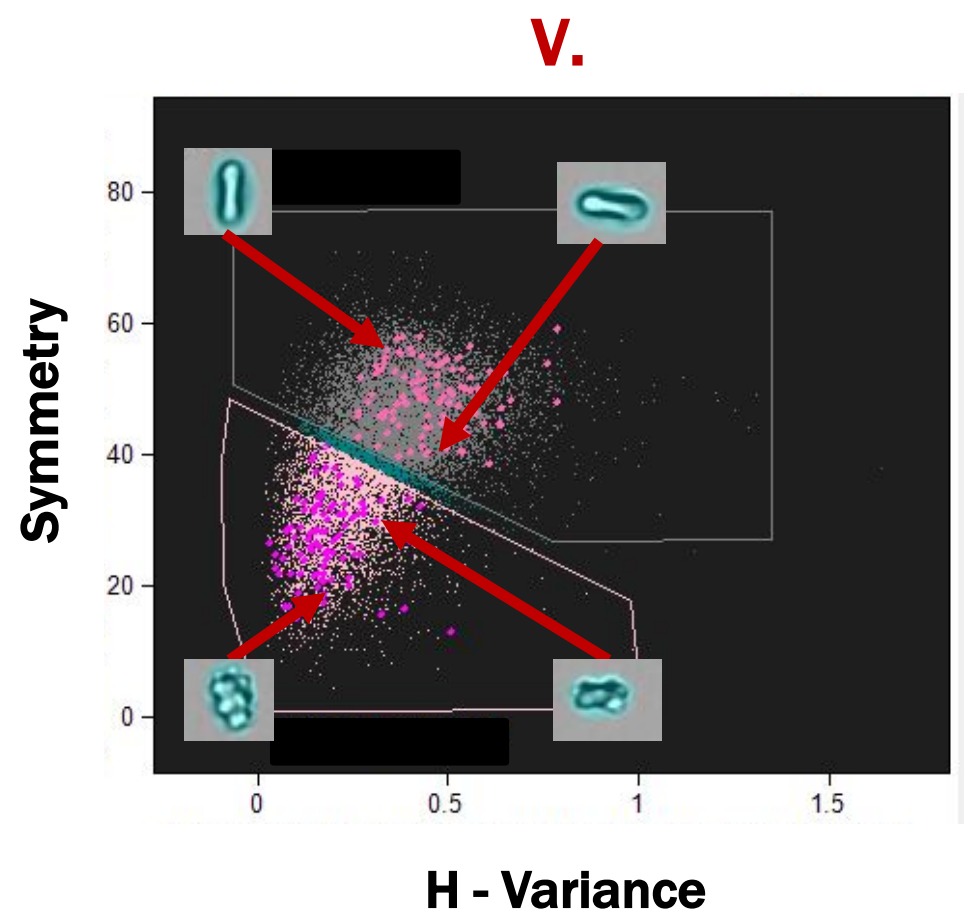

Figure 3-9: (see step V, Figure 3-4); plot of Symmetry versus H-variance, using the watershed mask. Inset images provide examples of side view discocyte (top) and side view spheroechinocyte (bottom) RBC images. Object masks are highlighted in light blue. Red arrows point to the location of images within the scatter plot. Feature finder/Truth populations were used to determine best features; overlaid on the sample population are truth populations for spheroechinocytes (purple) and discocytes (pink). A gate based on images within both ROIs (pink/grey boundary) were selected for analysis. 


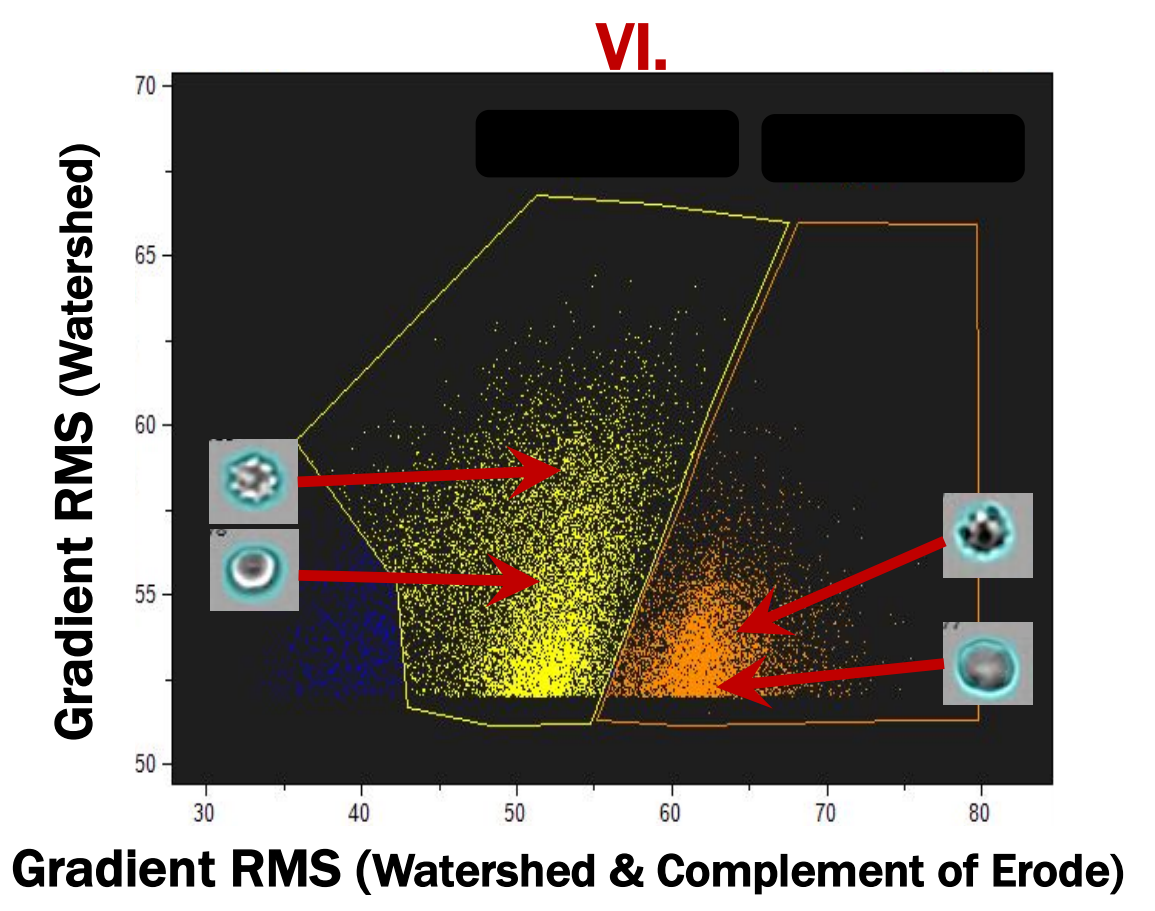

Figure 3-10: (see step VI, Figure 3-4); plot of Gradient RMS for a watershed mask versus a watershed AND NOT erode mask. Inset images provide examples of brighter shaded (left) and darker shaded (right) RBC images. Object masks are highlighted in light blue. Red arrows point to the location of images within the scatter plot. A gate based on images within both ROIs (yellow/orange boundary) were selected for analysis.

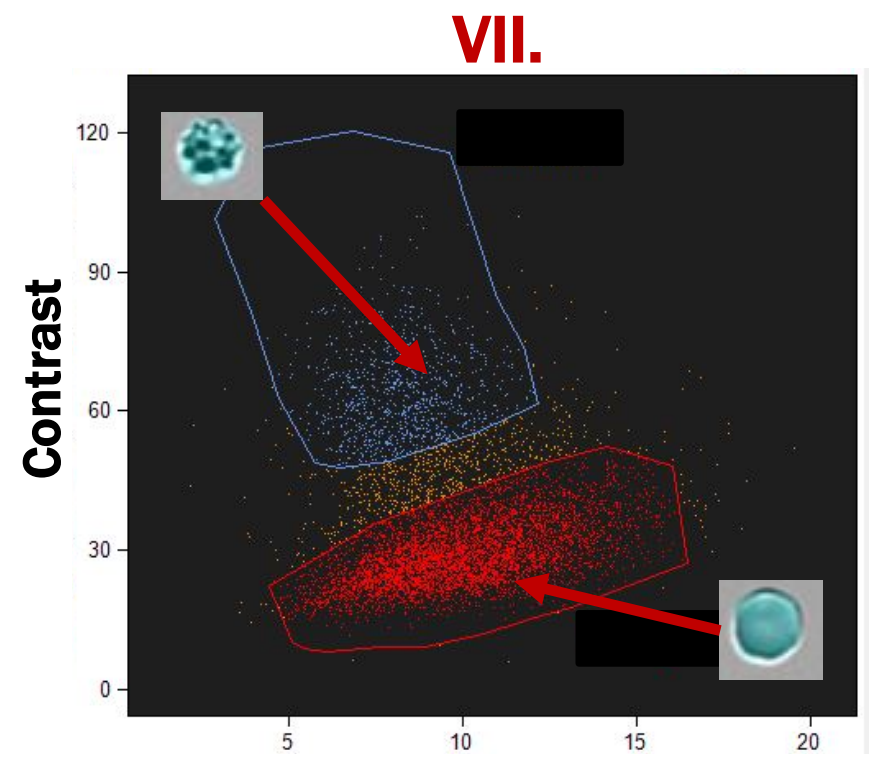

\section{H-Variance}

Figure 3-11: (see step VII, Figure 3-4); plot of Contrast versus H-Variance, using the watershed mask. Inset images provide examples darker shaded spheroechinocyte (top left) and discocyte (bottom right) RBC images. Object masks are highlighted in light blue. Red arrows point to the location of images within the scatter plot. Feature finder/Truth populations were used to determine best features; truth populations not overlaid in figure for simplicity. A gate based on images within both ROIs (red/blue boundary) were selected for analysis. 


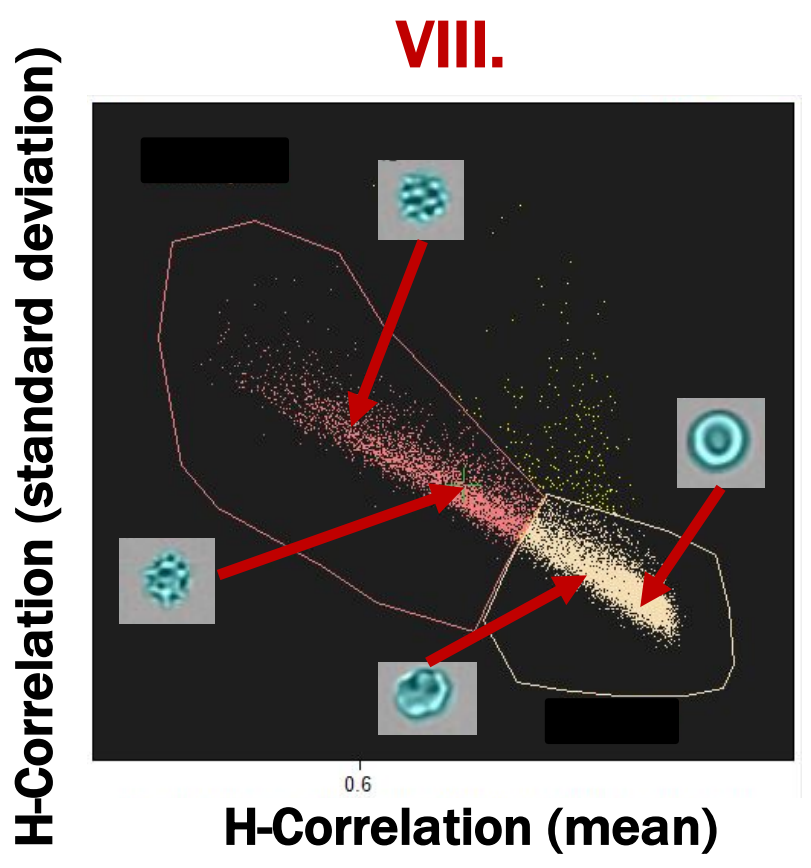

Figure 3-12: (see step VIII, Figure 3-4); plot of H correlation standard deviation versus H-correlation mean, using the watershed mask. Inset images provide examples of brighter shaded spheroechinocyte (left) and discocyte (right) RBC images. Object masks are highlighted in light blue. Red arrows point to the location of images within the scatter plot. Feature finder/Truth populations were used to determine best features; truth populations not overlaid in figure for simplicity. A gate based on images within both ROIs (pink/beige boundary) were selected for analysis.

\subsection{Spheroechinocyte Percentage Changes versus $\mathrm{SO}_{2}$ Changes in Stored RCCs}

To investigate the change in morphology distribution and $\mathrm{SO}_{2}$ changes of stored RCCs as a function of time, seven blood bags were monitored by extracting three samples from each bag for implementation into FLEX and IS ${ }^{\mathrm{X}} / \mathrm{RBC}$-MIST protocols (Sections 2.7.1-2.7.3). Raw data collected from these measurements are provided in Figure 3-13.

Figure 3-14 displays the percentage change, with respect to the initial value, of $\mathrm{SO}_{2}$ and spheroechinocyte population of the seven bags over their lifespan. Figure 3-15 is a Bland-Altman plot of the percentage change, with respect to the initial value, of $\mathrm{SO}_{2}$ and spheroechinocyte population of all seven bags over their lifespan. 


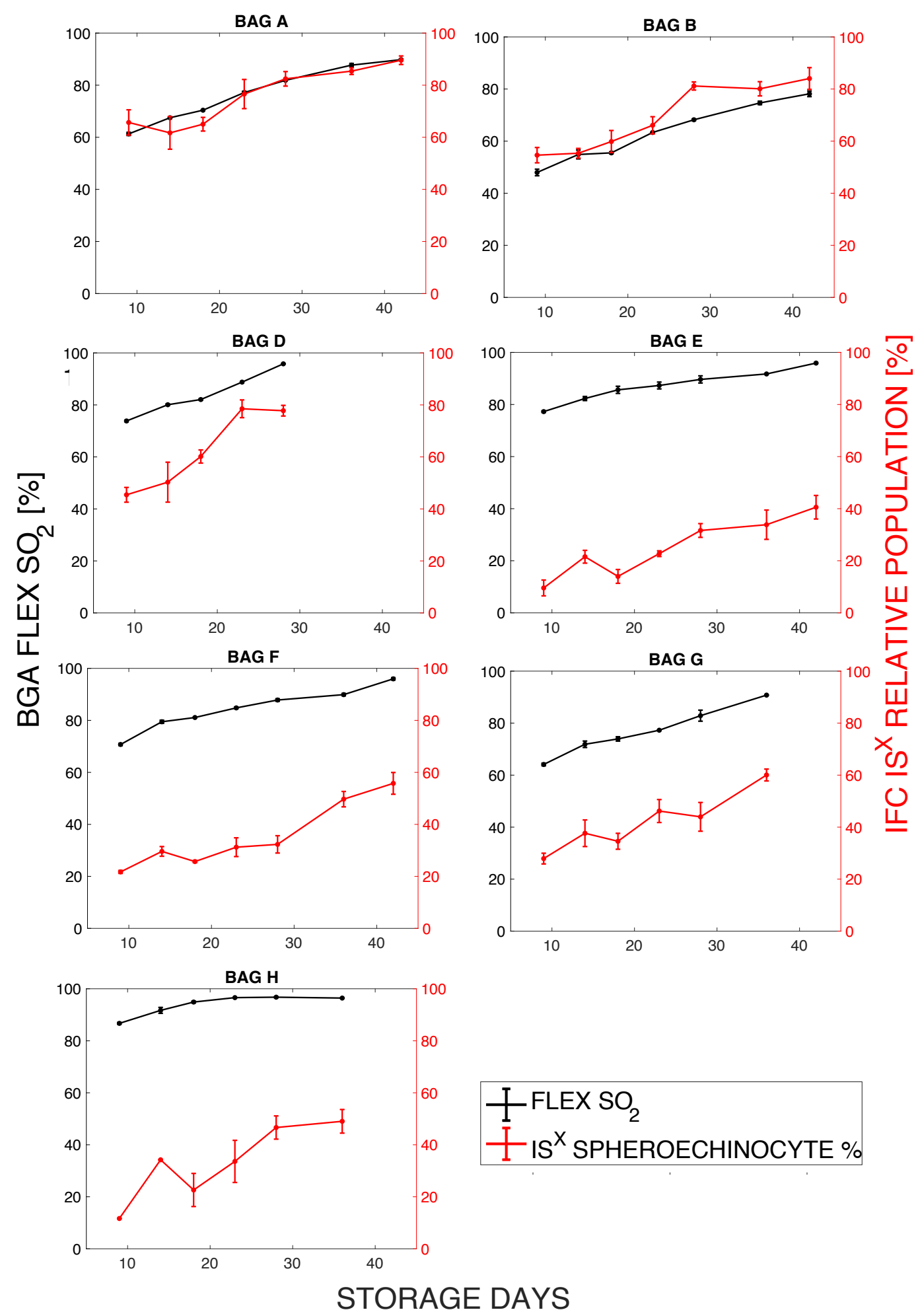

Figure 3-13: Plot of FLEX $\mathrm{SO}_{2}$ (black) and IS ${ }^{\mathrm{X}}$ Spheroechinocyte population percentage (red) mean values for RCC samples of Bags A,B,D,E-H over their 6 week lifespan. Bars indicate standard deviation of values obtained from three samples. 


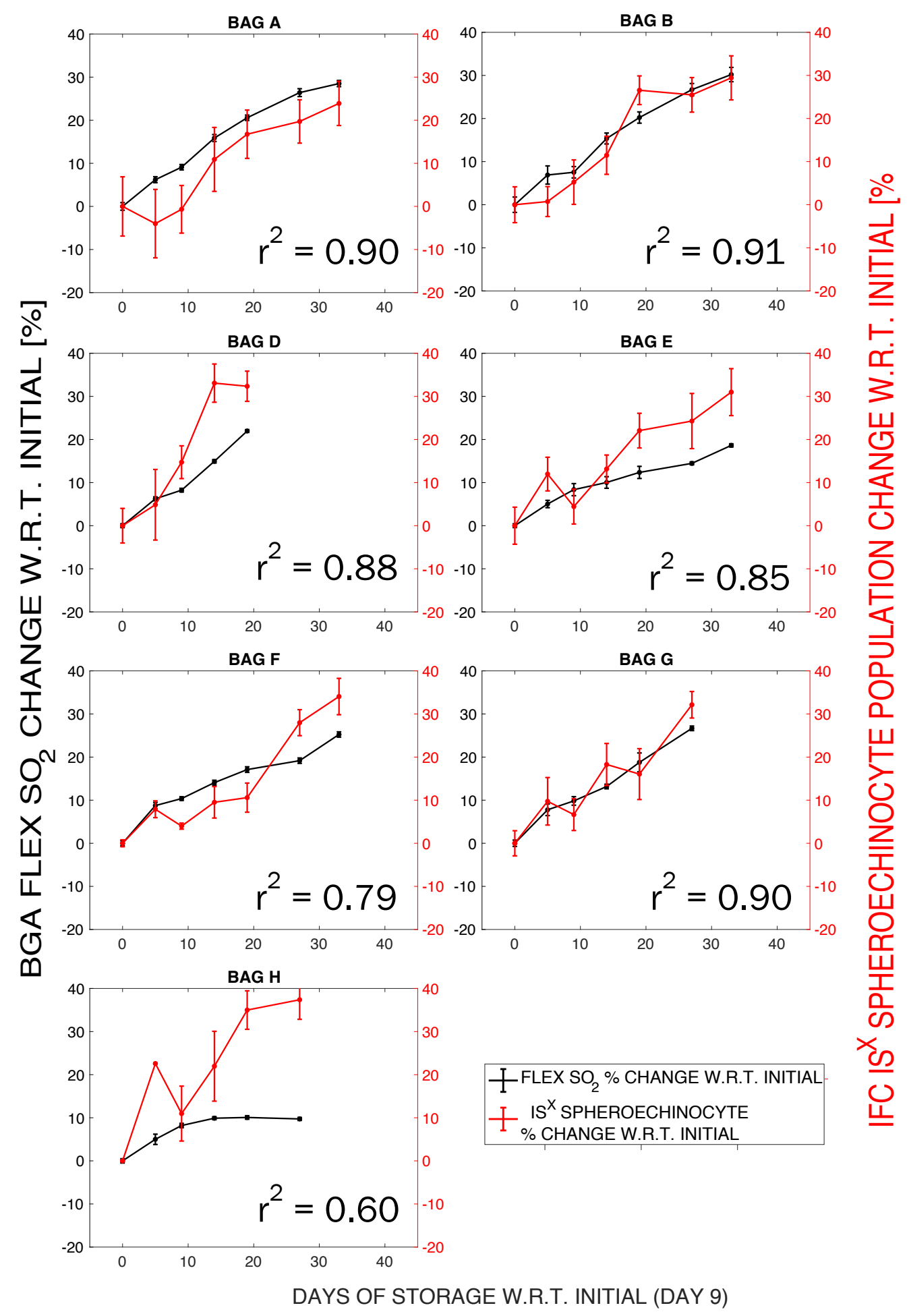

Figure 3-14: Plot of mean percentage changes of FLEX $\mathrm{SO}_{2}$ (black) and $\mathrm{IS}^{\mathrm{X}}$ Spheroechinocyte population (red) for RCC samples of Bags A,B,D,E-H over their 6 week lifespan. Changes are calculated with respect to initial (Day 9) measurement. Bars indicate (error-propagation accounted) standard deviation of values obtained from three samples. $r^{2}$ values calculated as the square of correlation coefficient. 


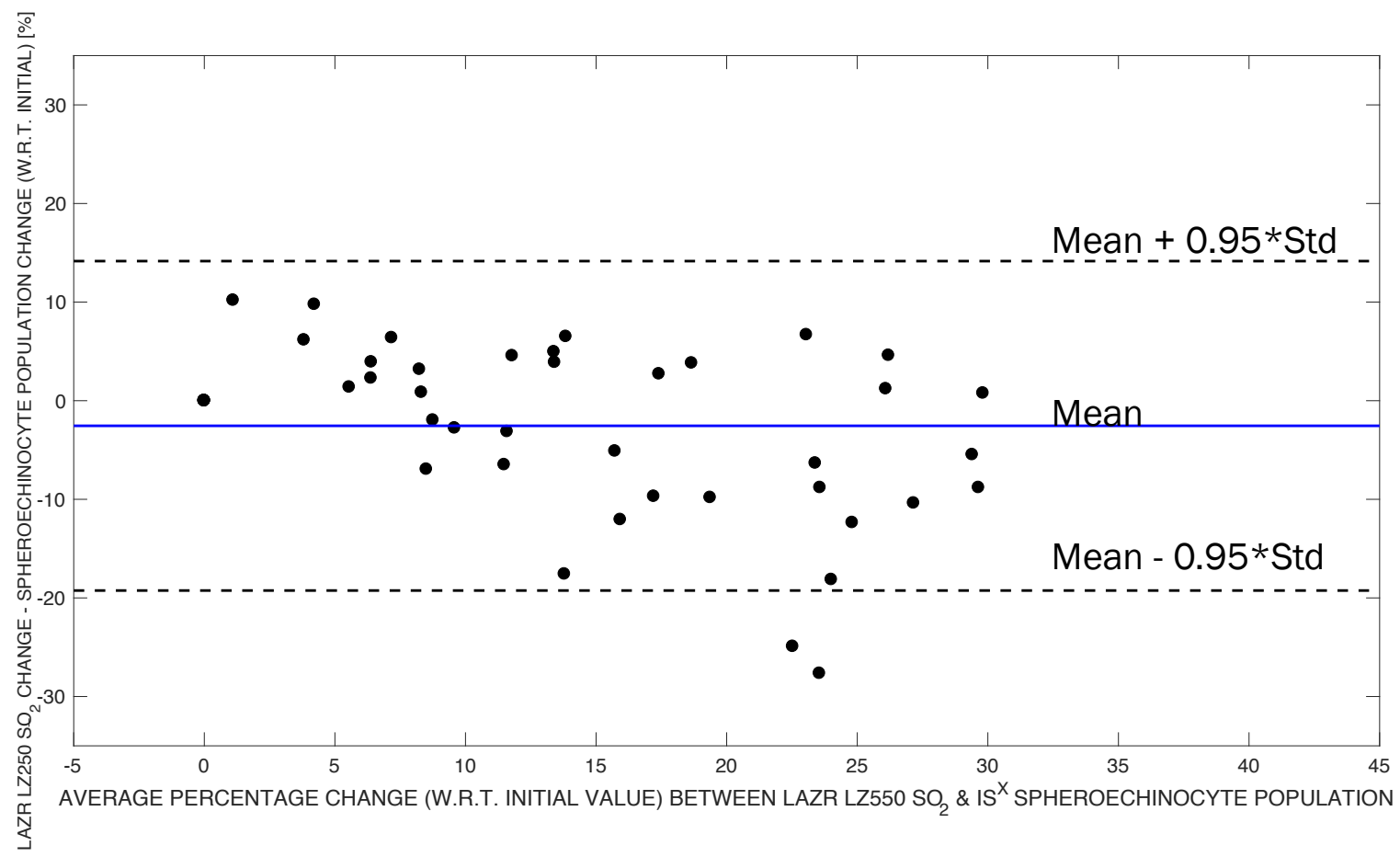

Figure 3-15: Bland-Altman plot of $\mathrm{SO}_{2}$ and Spheroechinocyte population percentage change including Bags A,B,D,E-H over their 6 week lifespan.

\subsection{Measurements of $\mathrm{SO}_{2}$ : in vitro versus in situ}

The same seven blood bags were monitored in situ using the LAZR setup and protocol (2.7.4). $\mathrm{SO}_{2}$ data collected from both PA transducers are plotted against the in vitro $\mathrm{FLEX} \mathrm{SO}_{2}$ values in Figure 3-16. Bland-Altman plots of each pair-combination of data sets (BGA-LZ250, BGALZ550 \& LZ250-LZ550) are displayed in Figure 3-17, 3-18 and 3-19. Mean values of all blood bags were used for the analysis. 


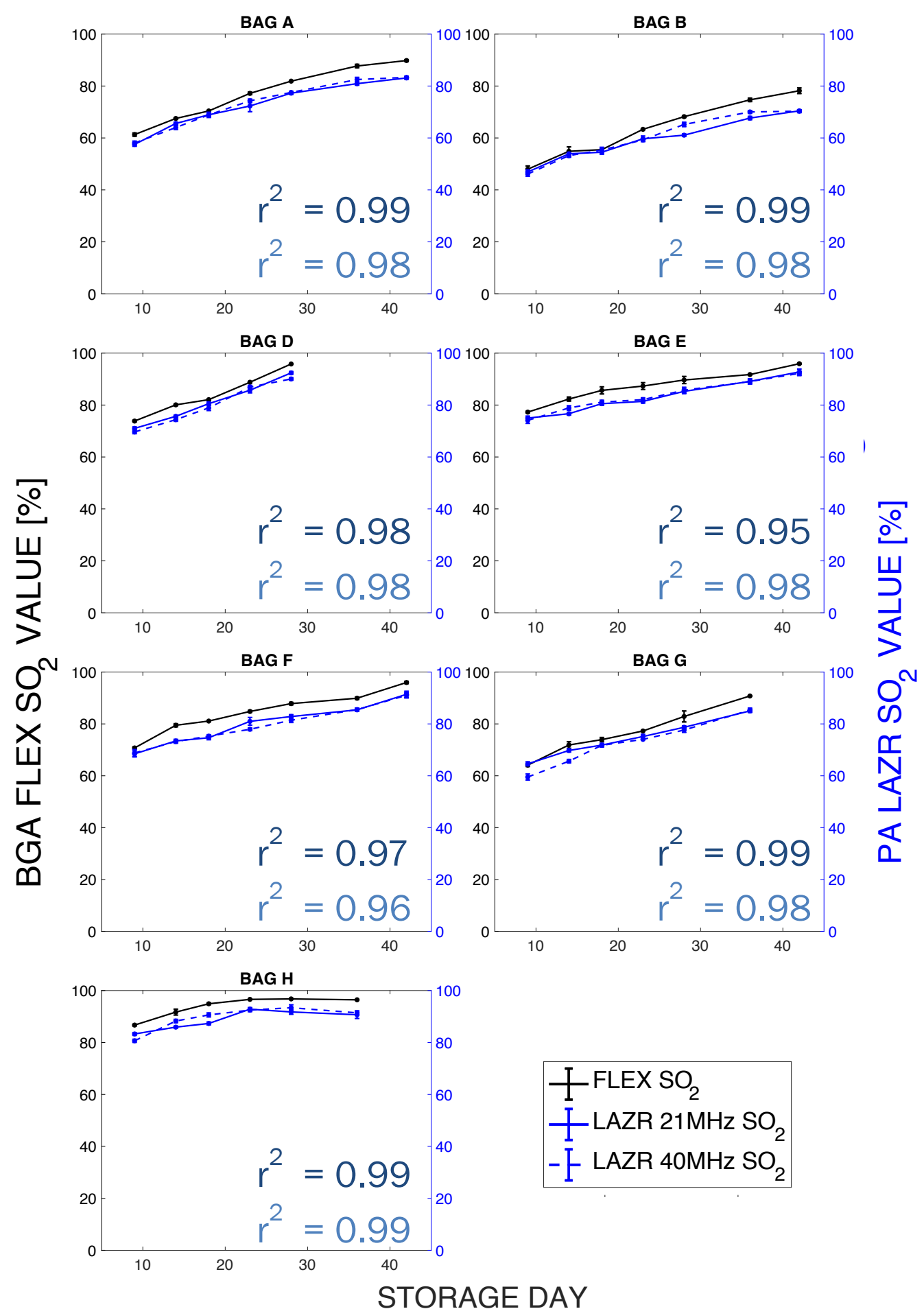

Figure 3-16: Plot of LAZR in situ $\mathrm{SO}_{2}$, for LZ250 (solid blue) and LZ550 (dashed blue) transducers, against FLEX $\mathrm{SO}_{2}$ (black) $\mathrm{SO}_{2}$ mean values for extracted RCC samples and Bags A,B,D,E-H over their 6 week lifespan. Bars indicate standard deviation of values obtained from three ROIs/samples. $\mathrm{r}^{2}$ values calculated as the square of correlation coefficient. 


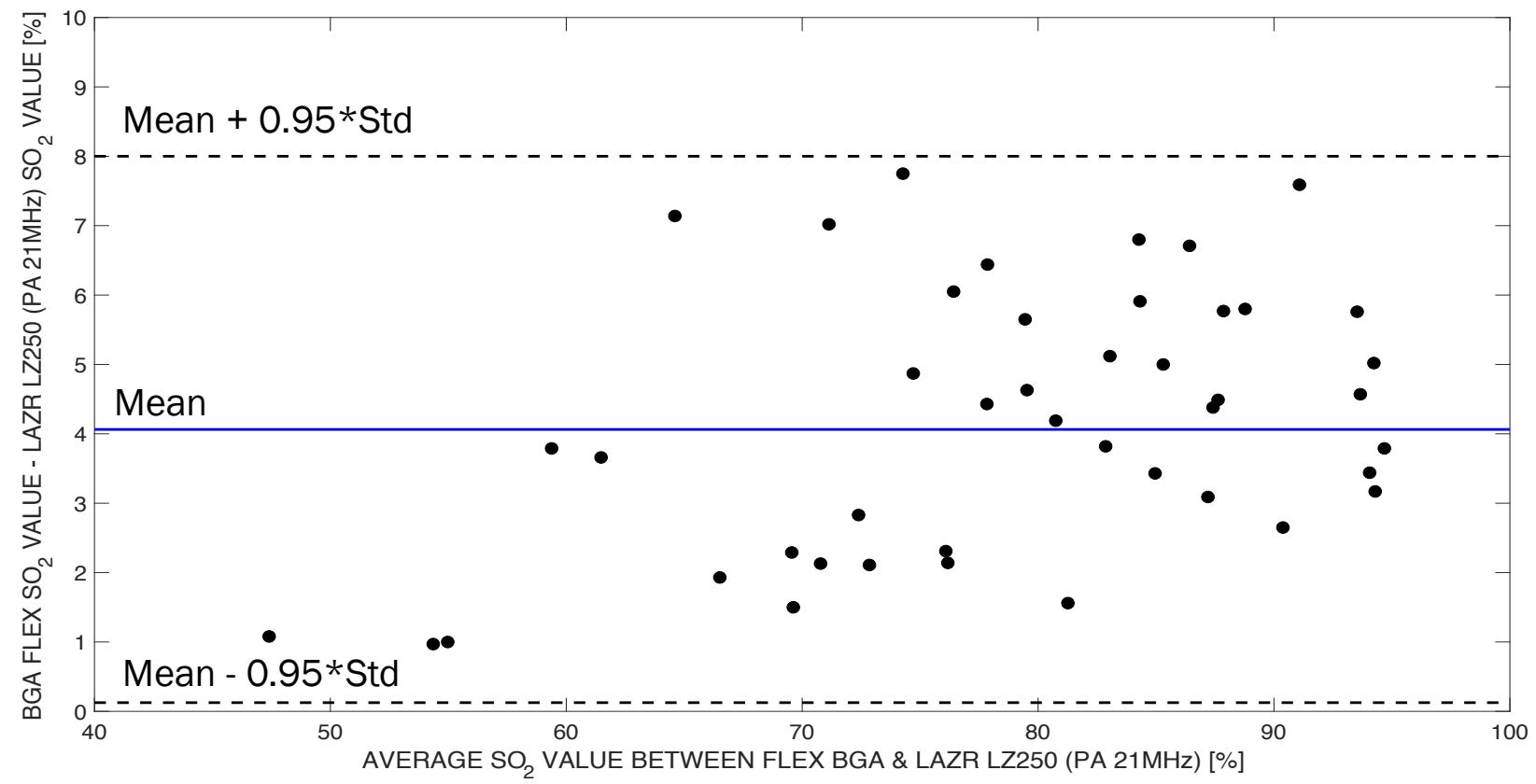

Figure 3-17: Bland-Altman plot of in situ LAZR LZ250 and in vitro FLEX SO 2 measurements for Bags A,B,D,E-H over their 6 week lifespan.

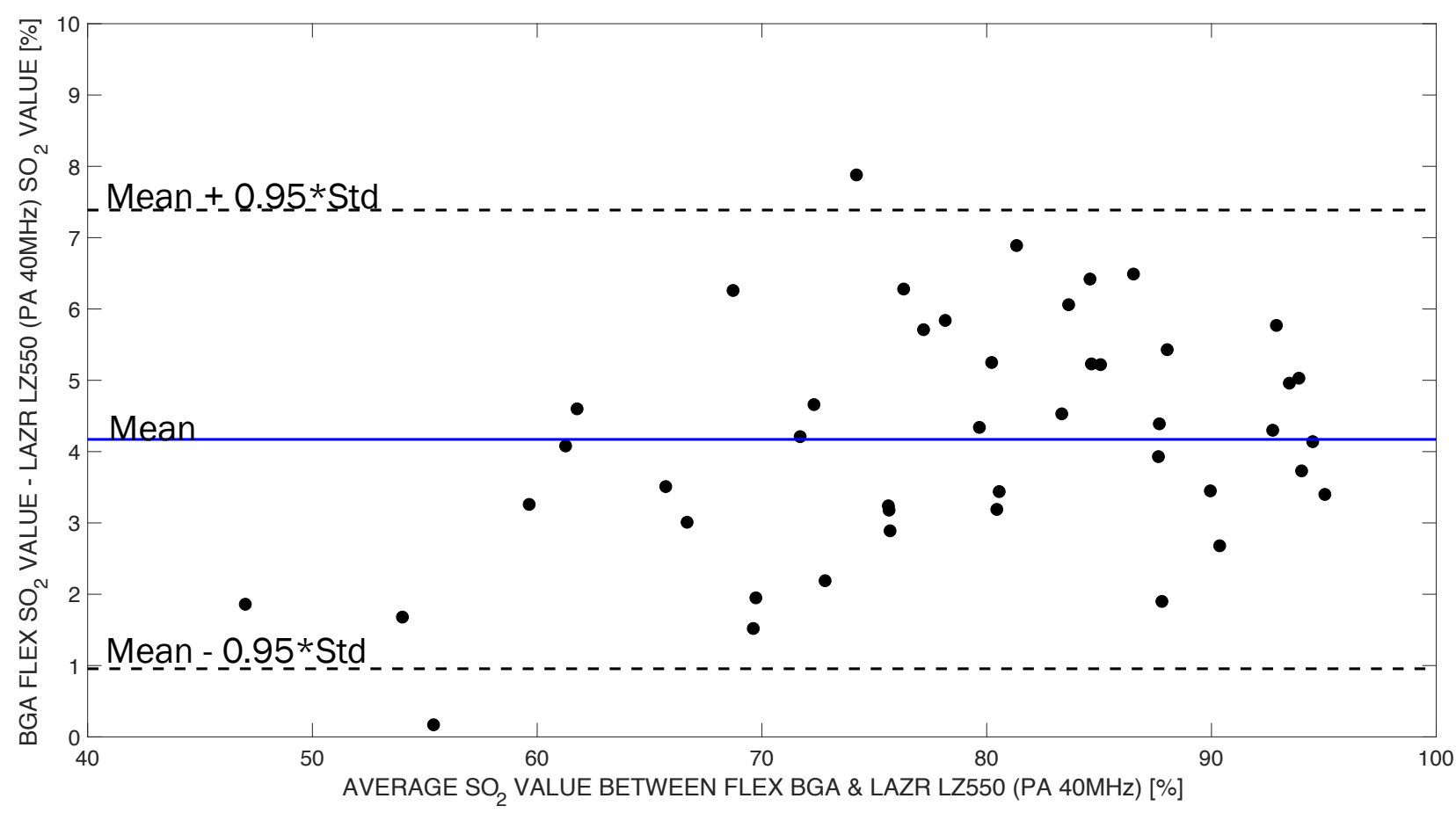

Figure 3-18: Bland-Altman plot of in situ LAZR LZ550 and in vitro FLEX $\mathrm{SO}_{2}$ measurements for Bags A,B,D,E-H over their 6 week lifespan. 


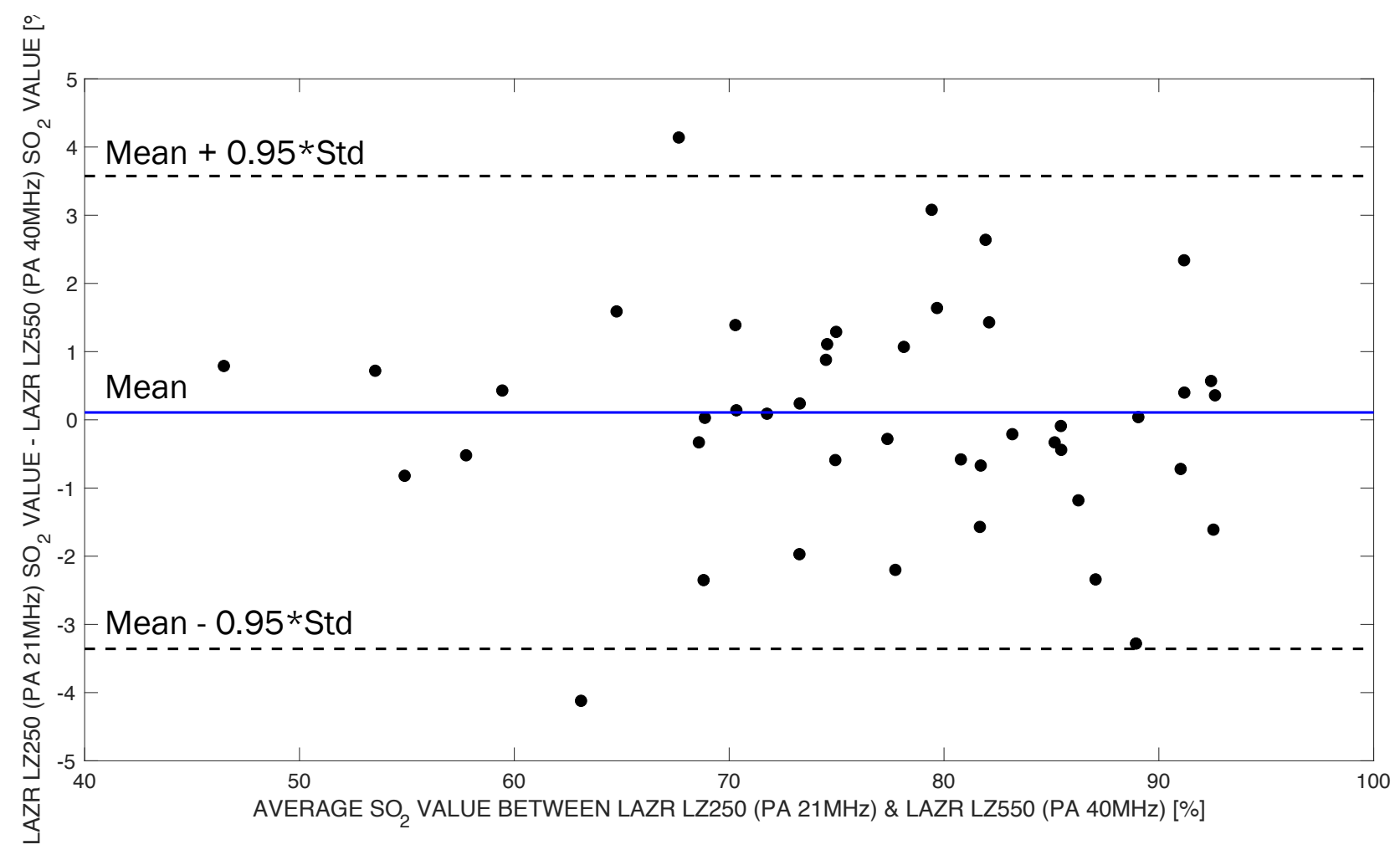

Figure 3-19: Bland-Altman plot of in situ LAZR LZ250 and LAZR LZ550 measurements for Bags A,B,D,E-H over their 6 week lifespan.

\subsection{Changes in RCC Morphology Distribution and in situ $\mathrm{SO}_{2}$ values of Blood Bags}

Figure 3-20 compares the changes in $\mathrm{SO}_{2}$ values obtained from the LAZR measurements with both transducers, against the $\mathrm{IS}^{\mathrm{X}}$ morphology distribution percentage changes. FLEX $\mathrm{SO}_{2}$ changes were superimposed to provide a reference of comparison from Figure 3-14. BlandAltman plots of each pair of data sets (IS ${ }^{\mathrm{X}}$-LZ250 \& IS ${ }^{\mathrm{X}}$-LZ550) are displayed in Figures 3-21 and 3-22 respectively. Mean values of all blood bags were used for the analysis. 


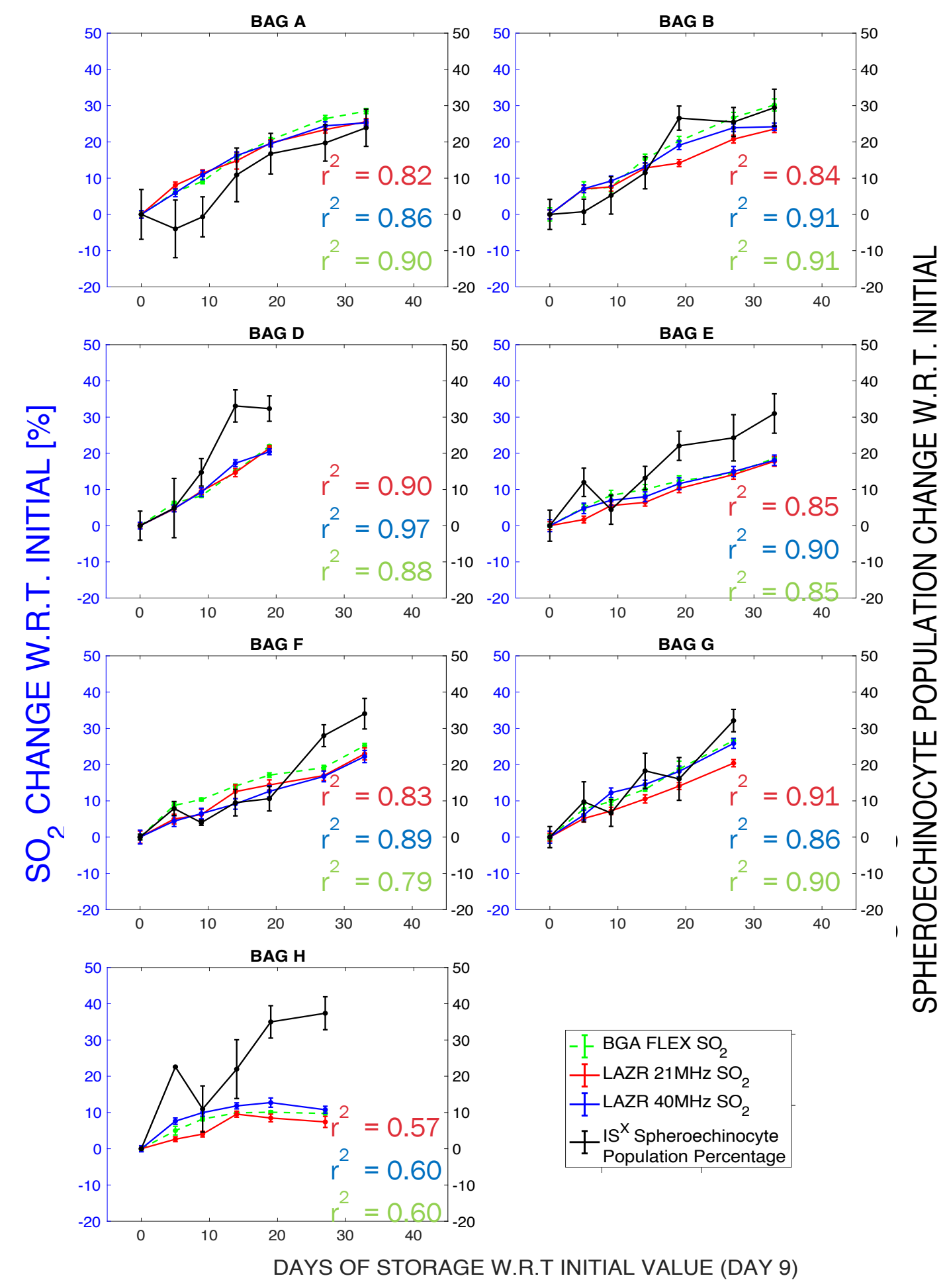

Figure 3-20: Plot of LAZR in situ $\mathrm{SO}_{2}$ changes for LZ250 (red) and LZ550 (blue) transducers, against ISX spheroechinocyte population changes (black) for extracted RCC samples and Bags A,B,D,E-H over their 6 week lifespan. FLEX SO 2 changes (green dashed) superimposed for comparison. Bars indicate standard deviation of values obtained from three ROIs/samples. $r^{2}$ values calculated as the square of correlation coefficient. 


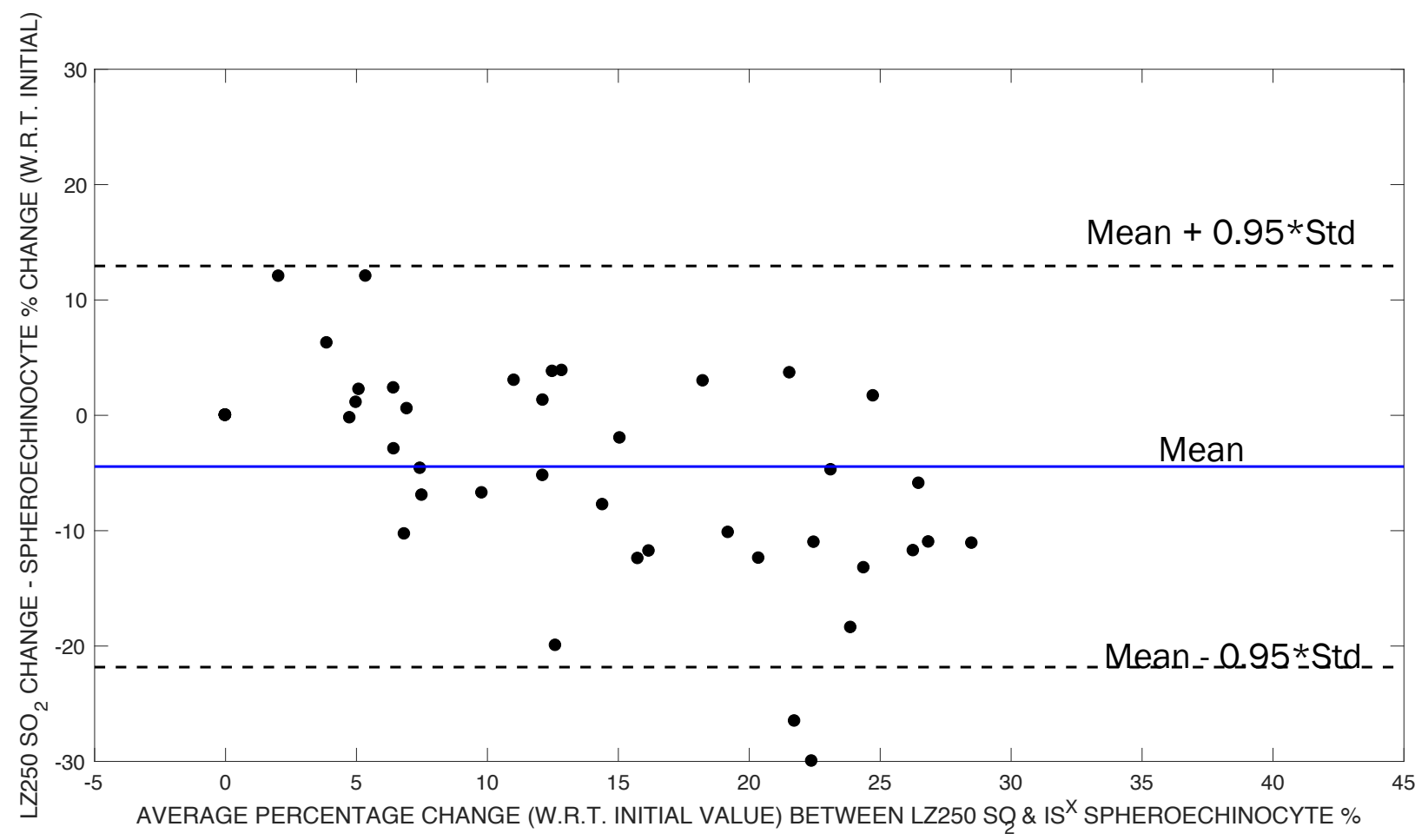

Figure 3-21: Bland-Altman plot of in situ LAZR LZ250 $\mathrm{SO}_{2}$ changes and in vitro ISX spheroechinocyte population percent changes for Bags A,B,D,E-H over their 6 week lifespan.

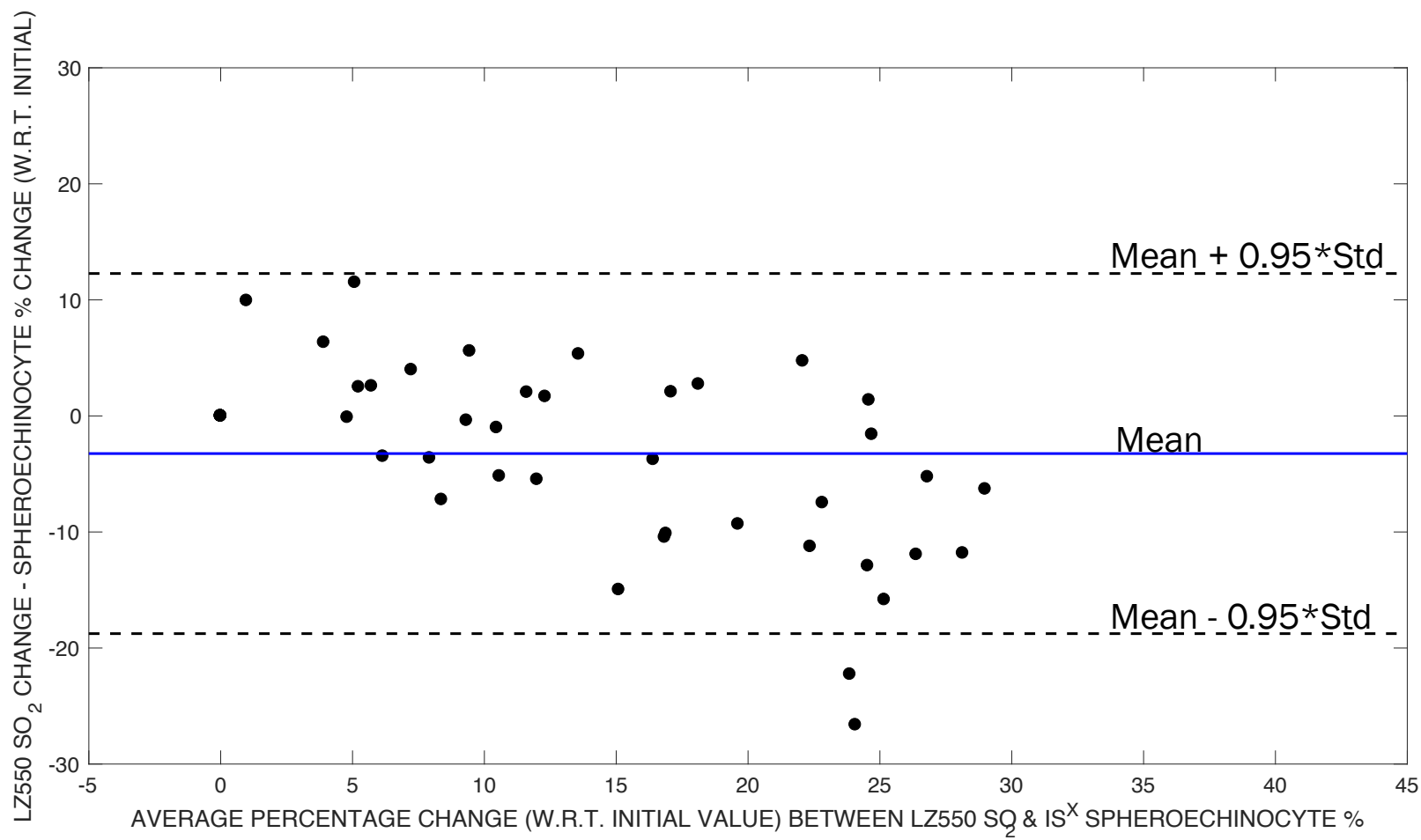

Figure 3-22: Bland-Altman plot of in situ LAZR LZ550 $\mathrm{SO}_{2}$ changes and in vitro ISX spheroechinocyte population percent changes for Bags A,B,D,E-H over their 6 week lifespan. 


\section{Chapter 4 : Discussion}

This chapter begins with a discussion of sections constituting the RBC-MIST, and how error analysis of such a template has been accomplished during the development (Section 4.1). The results using this template (namely spheroechinocyte population percentages) have been shown strongly correlate with $\mathrm{BGA} \mathrm{SO}_{2}$ changes over the lifespan of the blood bags (Section 4.2). Control experimental results are discussed, suggesting that the blood bag plastic produces negligible PA signals, and that $\mathrm{PA} \mathrm{SO}_{2}$ acquisition agrees with the gold standard for a large range of $\mathrm{SO}_{2}$ values (Section 4.3). While recognizing the very strong correlation between PA in situ $\mathrm{SO}_{2}$ changes and BGA in vitro $\mathrm{SO}_{2}$ changes, Section 4.4 also discusses factors contributing to the observed discrepancy in the measured values between the two methods. The chapter closes with a discussion on proposed reasons behind observed blood bag contaminations found during the end stages of their lifespan (Section 4.5).

\subsection{RBC-MIST}

The goal of the RBC-MIST was to create an image segmentation template, based on a series of mask/feature combinations, to objectively, rapidly and automatically determine the subpopulation percentage of spheroechinocytes within a sample of RCC (whose morphological traits are defined in Table 1-2). In general, feature values for individual images were calculated from the intensity distribution of pixels enclosed by an underlying object mask (Section 2.4.2). A plot of one or two image-related features was created to discriminate between subpopulations (Section 2.4.3). For steps I-IV of the RBC-MIST (Figure 3-3), features were manually chosen to discriminate between the intended subpopulations by visual verification.

As shown in Figure 3-5, a gradient RMS feature was used to extract focused images for further analysis. As gradient RMS measures the slope of pixel intensity changes, the object boundary (cell membrane) in a focused image would exhibit a larger gradient over a smaller range of pixels. On the other hand, unfocused or blurry images lacked large changes in intensity around the object boundary, thereby producing lower gradient $R M S$ values. The threshold value of 55 was chosen from visual verification. 
From these focused images, single cell images were then selected (Figure 3-6). The default bright field mask was unable to segment object aggregates within the same image, therefore grouping the objects in multi-cell images under one mask. The mask area for multiple cells would comparably be larger than that of individual cells. Furthermore, multi-cell object masks would generally exhibit an oblong shape compared to single cells, resulting in a lower value for the minor to major axis ratio (aspect ratio). As seen in Figure 3-6, multi-cell images with larger areas and smaller aspect ratios were found in the lower right corner of the plot.

While multi-cell images were excluded from further analysis (Figure 3-6), a small but significant fraction of object images containing calibration beads (speed beads) were not successfully removed (image C, step II, Figure 3-3). The presence of these beads impaired mask application and subsequent feature analysis and therefore needed to be removed. To address this issue, an erode function was applied to shrink the bright field mask to only cover the brightest regions of the image. For images containing cells and calibration beads, this resulted in two disjoint masks encompassing each object. The feature spot count was used to count the number of disjoint masks within an image, yielding a value of 2 for images containing a cell and bead. In this manner, Figure 3-7 illustrates the selection of single cell image subpopulations exhibiting only one mask.

A watershed mask, applied with an adaptive erode function, was used to contour the object to capture more detail on the cell boundary (Table A-3). This mask was applied for the discrimination of front view from side view images, where the circularity feature was plotted against aspect ratio (Figure 3-8). Front view images were expected to exhibit a closer resemblance to a circle, and have a minor to major axis ratio closer to 1 . As evident in the inset images of Figure 3-8, front view images with larger aspect ratio and circularity values were found in the top right corner of the plot.

A mask combination similar to that of Figure 3-8 was applied for the discrimination of lighter and darker shaded images (Figure 3-9). For brighter shaded cell images, intensity changes around the entire cell exhibited larger gradients compared to darker shaded images; however, at the cell boundaries, gradient changes were larger for darker shaded images. With these 
discrepancies in mind, the gradient $R M S$ feature was considered as a good candidate to differentiate between these subpopulations. The feature was applied with a mask that covered the entire cell (watershed) and with a mask covering the cell boundary (watershed intersected with the complement of erode). Hence, darker shaded images were able to be segregated from the brighter shaded images using the two masks.

For steps V, VII and VIII (Figure 3-4), the feature finder/truth population method (Section 2.4.3) was used to find the ideal features to optimally discriminate subpopulations. Firstly, approximately 50-100 images defining truth populations representative of RBC subtypes were chosen in consultation with an RBC morphology expert (T. Turner, COI, CBS, Edmonton). Using the truth populations, the feature finder calculated the $\mathrm{RD}$ mean (Fisher's discriminant ratio) - the difference in the means divided by the sum of the standard deviations for the two populations - for each feature [207]. Features with higher RD values equated to higher discriminatory power and hence better subpopulation separation. Once these features were plotted against one another (for the sample population), the truth populations were overlaid to help refine subsequent gates and ROIs. This gating strategy was used to discriminate between side view (Figure 3-9), darker shaded (Figure 3-11) and brighter shaded (Figure 3-12) spheroechinocyte and discocyte subpopulations.

Error analysis of the RBC-MIST was not carried out quantitatively for multiple reasons. Firstly, the development of the RBC-MIST included frequent qualitative error analysis. Viewing images of any data point within the plots allowed for a visual feedback mechanism resulting in the refinement of ROIs. Furthermore, the ability to combine masks and functions, manipulate features and modify existing ROIs made improving the template more convenient. Secondly, a quantitative error analysis of the $\mathrm{RBC}$ image subpopulations would inevitably require a subjective and impractical verification; the gold standard verification technique would require an RBC morphology expert to peruse through tens of thousands of images to quantify error.

Even if this resource-intensive, subjective task were to be carried out, the RBC-MIST template assigns multiple subpopulations into either discocytes or spheroechinocytes. Hence, the error analysis would also have to account for images that were incorrectly assigned to a subpopulation while being correctly assigned to discocytes or spheroechinocytes. It is suspected that the error 
pertaining to images that did not satisfy both criteria would be small, occurring mostly at the crenated discoid/crenated spheroid boundary. This boundary ambiguity has been mitigated for by introducing multiple pathways in the RBC-MIST (Figure 3-4) to carefully discriminate between the two subpopulations. Although standard quantitative error analysis may not be suitable for the RBC-MIST, a method still needs to be developed for a comparison and/or validation against the light microscopy gold standard technique (Section 1.7.1). Suggestions for future work on this matter is discussed in Section 5.1.

\subsection{Spheroechinocyte Percentage Changes versus $\mathrm{SO}_{2}$ Changes in Stored RCCs}

The raw datasets of $\mathrm{SO}_{2}$ and morphology distribution percentage for all seven blood bags stored for 6 weeks are displayed in Figure 3-13. The relative population percentages of spheroechinocytes vary in the initial value as well as the rate of change over time. Similar variability is also experienced by $\mathrm{SO}_{2}$, however, the changes appear to be independent of the morphological parameter. Regardless of the initial value, both $\mathrm{SO}_{2}$ and spheroechinocyte population percentage increased over storage life for all bags. This trend is consistent with the structural and functional changes of RBC storage lesion (Section 1.5.1).

Bags A and B contained a spheroechinocyte population percentage exceeding $50 \%$ and resulted with an RBC storage lesion population of over $80 \%$ by the end of their shelf life, which would be highly unfavorable for transfusion. On the other hand, bag E started with a spheroechinocyte percentage of $10 \%$, increasing to only $40 \%$ by the end of shelf life. Since all bags were manufactured using the B1 filtration method (Section 1.3.3), the wide ranges of RBC storage lesions prevalence across the blood bags are suspected to be attributed due to donor variability.

Figure 3-14 investigated the trends between the changes of both $\mathrm{SO}_{2}$ and spheroechinocyte population percentage over time. A strong correlation was established in the rate of change between both parameters, with the exception of bag $\mathrm{H}\left(\mathrm{r}^{2}=0.60\right)$. Bag $\mathrm{H}$ had an initial $\mathrm{SO}_{2}$ in the mid 80\%s (Figure 3-13), and increased until it approached the FLEX's maximum detection value of $95-96 \%$, after which it was forced to plateau. Bag H's case sheds light on a limitation of this direct method of comparison between $\mathrm{SO}_{2}$ and morphology. The $\mathrm{x}$-axis of Figure 3-15 shows 
that the average $\mathrm{SO}_{2} /$ lesion population change over the lifespan of the bag can reach $30 \%$, implying that initial $\mathrm{SO}_{2}$ values significantly greater than $70 \%$ may undergo a plateauing effect as the values approach the detection limit of the BGA.

The Bland Altman graph in Figure 3-15 involved the average and differences between IFC and BGA measurements of all data points collected from all bags. The FLEX $\mathrm{SO}_{2}$ percent changes were on average $2.59 \%$ lower than the IS ${ }^{\mathrm{X}}$ spheroechinocyte population percentage changes. The standard deviation of the differences was $8.66 \%$, and over $95 \%$ of the data points fell within the 95\% Gaussian confidence interval.

The data discussed in this section supports the primary hypothesis that during blood bag storage changes in RBC storage lesion population are correlated with changes in RBC oxygen saturation. Published work characterizing $\mathrm{RBC}$ storage lesions has documented increases in spheroechinocyte population and $\mathrm{SO}_{2}$ over the in vitro storage period of RCCs; however, the results presented in this thesis have shown, for the first time, that a correlation exists between the changes in these two parameters. Although both parameters are consequences of ATP reduction and oxidative stress during in vitro storage (Section 1.4.1), their agreement in percent changes is not intuitive. This agreement is speculated to be more of an empirical observation, rather than the result of an underlying biochemical process.

This correlation suggests that by tracking $\mathrm{SO}_{2}$ changes, an inference can potentially made about the changes in RBC morphological distribution. Hence, along with the functional information that $\mathrm{SO}_{2}$ changes provide, structural information of the $\mathrm{RBC}$ storage lesion can also be inferred with this parameter.

\subsection{Control Experiments}

The contribution of the blood bag plastic to the PA signal was investigated using the apparatus outlined in Section 2.6.1. Figure 3-1 plots the absolute value of the Hilbert transform of an RF signal averaged from eleven elements. The signals produced by RCC compared to water in Figure 3-1 suggest that there is no discernable photoacoustic signal produced by the blood bag 
plastic cover. Furthermore, the location of the largest peak observed in the RCC plot (red curve, Figure 3-1) provides further evidence that PA signals are produced by the RCC and not the blood bag plastic.

The accuracy and repeatability of $\mathrm{SO}_{2}$ values acquired from the LAZR was tested via the experimental protocol outlined in Section 2.6.2. Figure 3-2 shows that $\mathrm{LAZR} \mathrm{SO}_{2}$ values closely match the gold standard BGA (FLEX) values for the hemoglobin samples. The results indicate that both the LZ250 and LZ550 probes (see Section 2.5.2) can be used to acquire accurate $\mathrm{SO}_{2}$

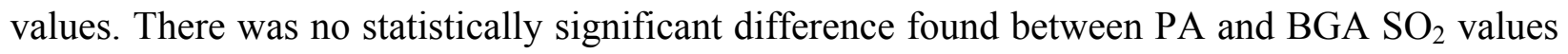
for any of the hemoglobin solutions, showcasing PA accuracy over a large range of $\mathrm{SO}_{2}$ values.

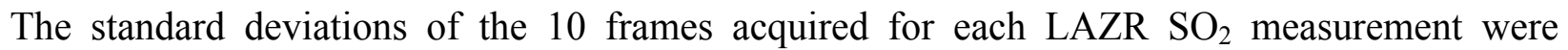
similar to that produced by 3 independent FLEX measurements. The similar standard deviations add more confidence in the accuracy and repeatability of $\mathrm{PA} \mathrm{SO}_{2}$ measurements.

\subsection{Measurements of $\mathrm{SO}_{2}$ : in vitro versus in situ}

The $\mathrm{SO}_{2}$ values produced by both in situ measurements of blood bags using the LAZR setup and the in vitro measurements of extracted RCC samples using the FLEX instrument are plotted against each other in Figure 3-16. A very strong correlation is observed between both transducer values and the BGA values, with the lowest $r^{2}$ value calculated to be 0.95 . The graph and correlation provide supporting evidence towards the secondary hypothesis that photoacoustic technology can acquire the $\mathrm{SO}_{2}$ of a blood bag in situ.

Figure 3-17 and Figure 3-18 showcase Bland Altman plots of the FLEX $\mathrm{SO}_{2}$ values against LAZR LZ250 and LZ550 $\mathrm{SO}_{2}$ values, respectively. A significant mean bias of $4.1 \%$ and $4.2 \%$ was observed for the mean differences between parameter values for both graphs. Standard deviations were $2.1 \%$ and $1.64 \%$ respectively and over $95 \%$ of data points fell within the Gaussian confidence interval, supporting strong correlation and a close agreement between the values of the methods of $\mathrm{SO}_{2}$ measurements. However, the fact that FLEX $\mathrm{SO}_{2}$ values were on average approximately 4\% greater than those derived from the PA method suggests that there is a systematic error. 
A hypothesis proposed to explain the discrepancy between FLEX and LAZR $\mathrm{SO}_{2}$ measurements was that in extracting the RCC samples from the blood bag, external oxygenation inevitably takes place, leading to an increase in $\mathrm{SO}_{2}$ for samples that are measured by the BGA. To test this hypothesis, LAZR measurements were taken of the safePICO syringes (Section 2.2.1) containing RCC samples, and compared against the $\mathrm{SO}_{2}$ values acquired from the same sample in the FLEX. An agreement between the data would provide evidence, beyond the control experimental results (Section 4.3), that PA can accurately determine $\mathrm{SO}_{2}$ values. Furthermore, it would also suggest that the LAZR $\mathrm{SO}_{2}$ values of blood bags in situ are lower than that measured in the syringe via LAZR and FLEX due to less exposure to the external environment.

Figure 4-1 provides an image of the setup established for the acquisition of $\mathrm{PA} \mathrm{SO}_{2}$ values from the syringe. The safeTIPCAP blocked water from entering the syringe, allowing for the submersion of the syringe into the blood bag container (Section 2.7.4). 3 syringes (each) were used to extract RCCs from 3 blood bags of varying oxygen saturation. The syringes were

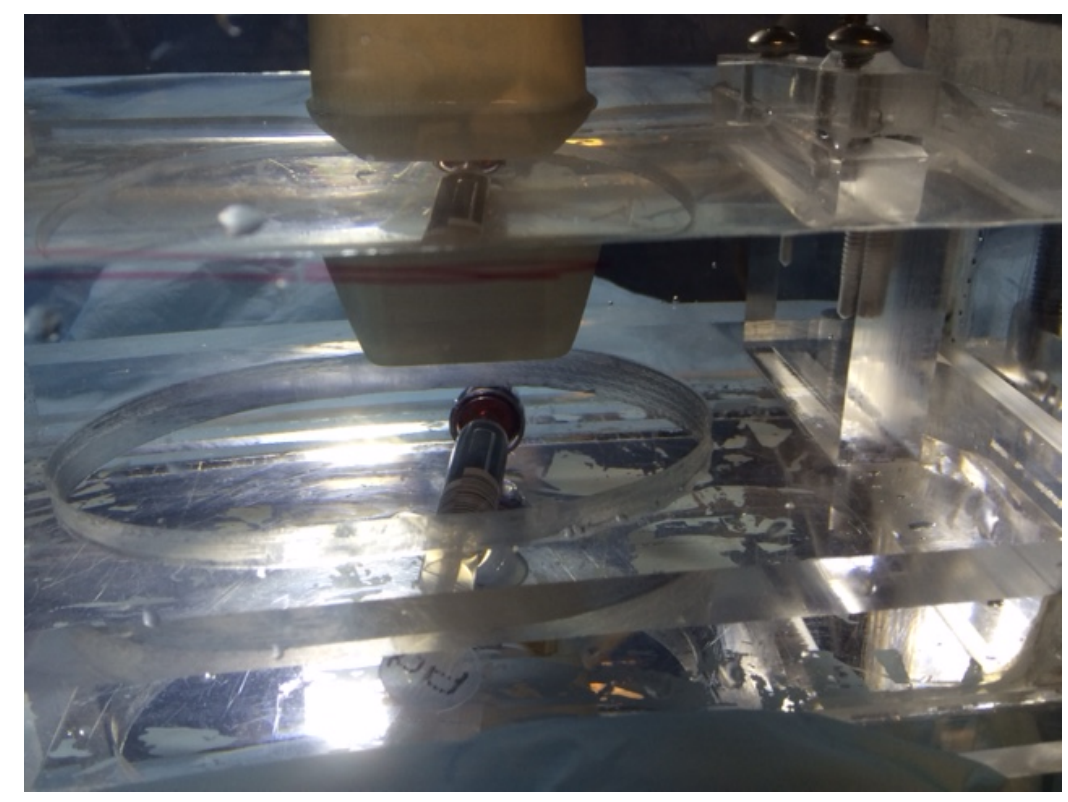

Figure 4-1: Setup developed to measure the PA SO2 value of extracted RCC samples from safePICO syringes, immediately after use in the FLEX.

measured by the FLEX, and immediately after, were placed into the LAZR setup; after the FLEX and LAZR acquisition, PA measurements of the blood bags were taken in situ. The entire experiment was then repeated for the other transducer. Figures 4-2 and 4-3 provide a $\mathrm{BGA} \mathrm{SO}_{2}$ 
versus $\mathrm{PA} \mathrm{SO}_{2}$ graph for three bags of varying $\mathrm{SO}_{2}$. The LAZR syringe (red) values are visibly higher in value compared to the LAZR blood bag SO2 values (blue), and are closer to the unity curve (dashed line).

The higher $\mathrm{PA} \mathrm{SO}_{2}$ values of RCC samples in a syringe compared to blood bags indicate that the syringe RCC is more oxygenated than the RCC within the bag. A close agreement between LAZR and FLEX $\mathrm{SO}_{2}$ measurements for the same syringe provides further validation of the technique. The experimental results further suggest that the lower $\mathrm{PA} \mathrm{SO}_{2}$ values observed in the blood bag may be primarily due to lack of external environment exposure.

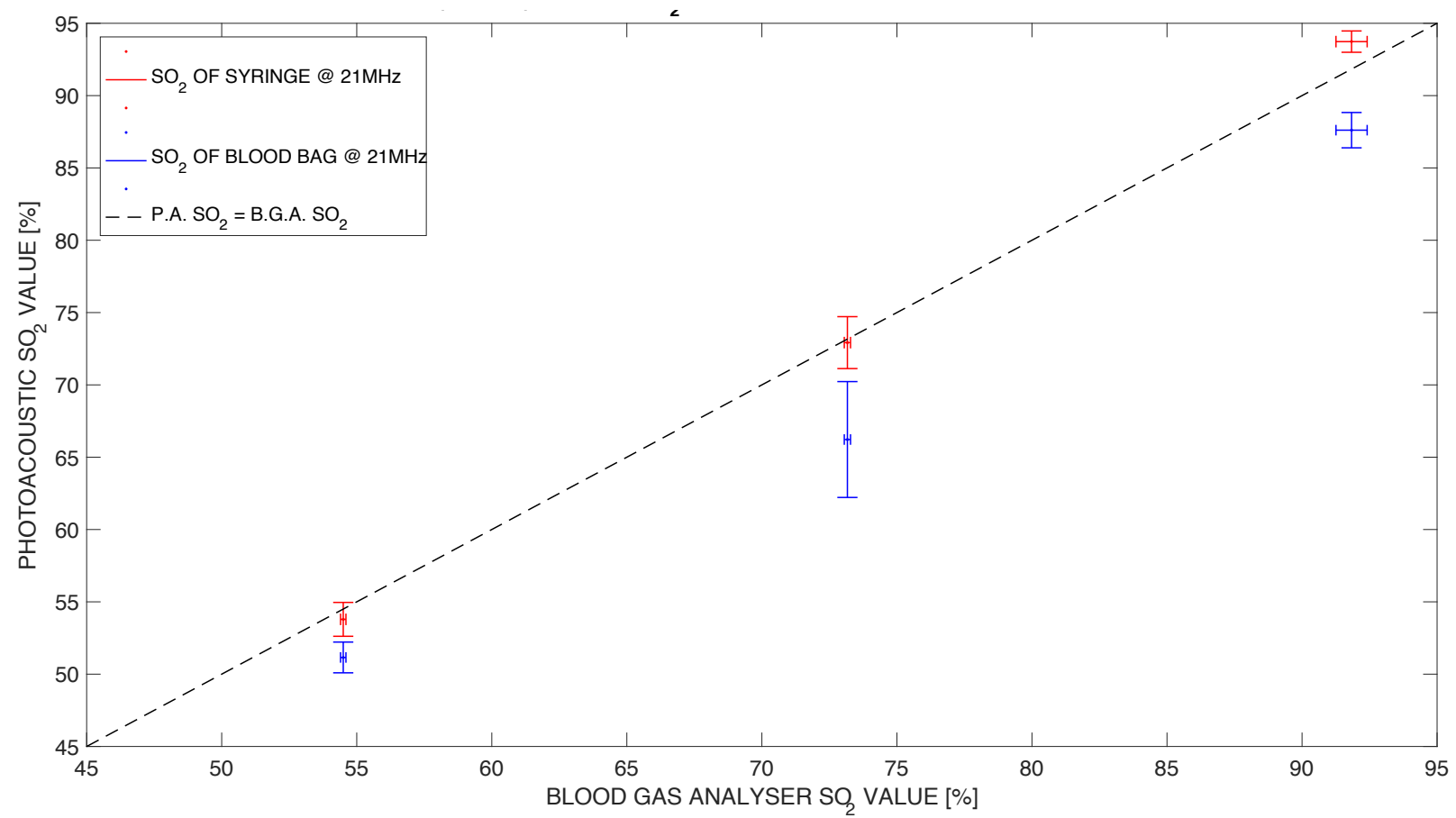

Figure 4-2: Syringe and in situ blood bag $\mathrm{SO}_{2}$ measurements acquired by the LAZR LZ250 (21MHz) against FLEX

$\mathrm{SO}_{2}$ values. Bags of varying oxygenation were used to investigate range of agreement. Dashed line refers to theoretical perfect agreement between BGA and PA. X/Y axis error bars refer to mean and standard deviation of three measurements (3 syringes/3 ROIs) per blood bag. 


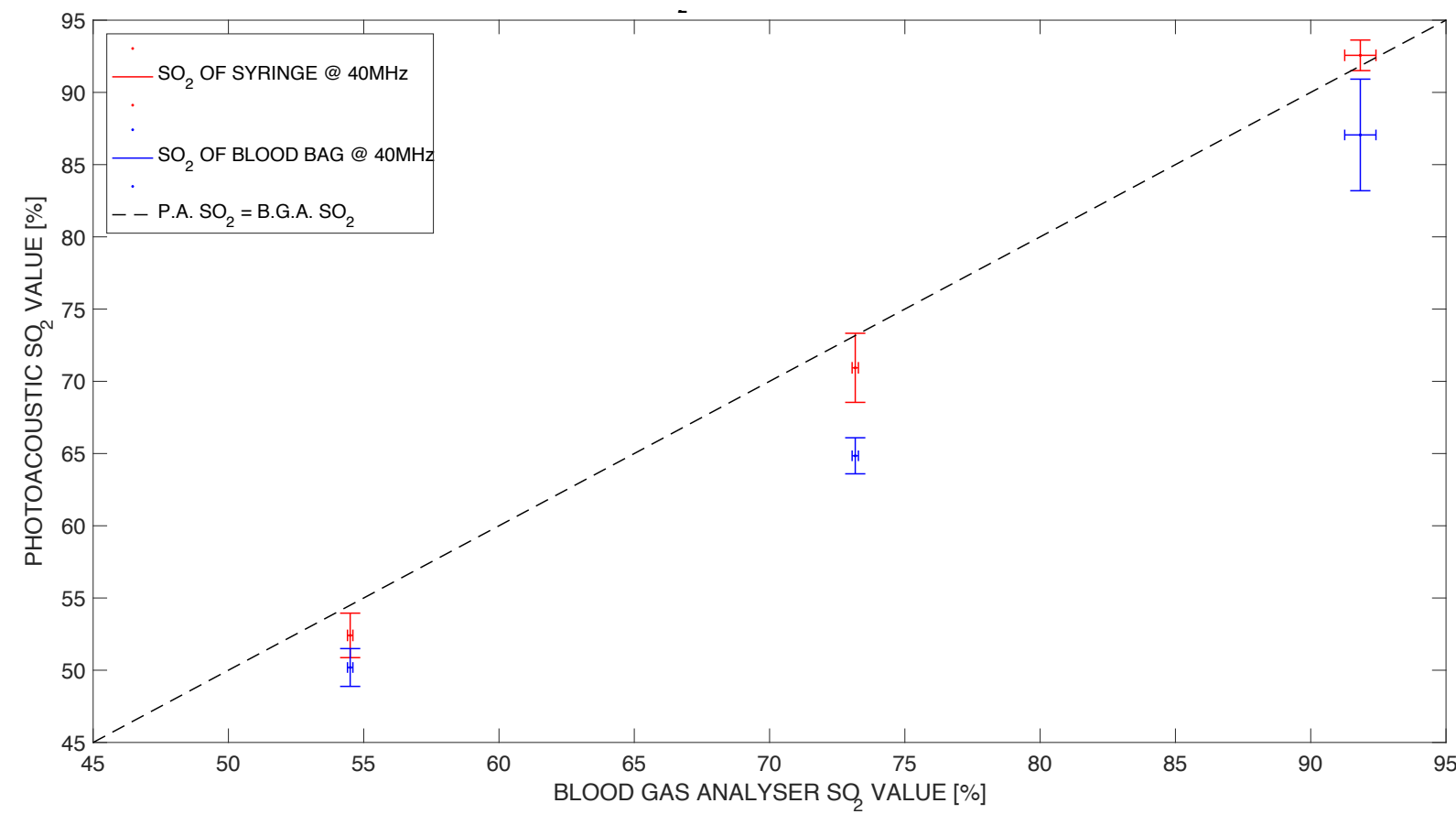

Figure 4-3: Syringe and in situ blood bag $\mathrm{SO}_{2}$ measurements acquired by the LAZR LZ550 (40MHz) against FLEX

$\mathrm{SO}_{2}$ values. Bags of varying oxygenation were used to investigate range of agreement. Dashed line refers to theoretical perfect agreement between $\mathrm{BGA}$ and $\mathrm{PA}$. X/Y axis error bars refer to mean and standard deviation of three measurements (3 syringes/3 ROIs) per blood bag.

\subsection{Issues Related to Blood Bag Contamination}

Out of the 8 blood bags used for thesis experiments, 3 of them had abrupt declines in $\mathrm{SO}_{2}$ values over a very short period of time. Figure 4-4 illustrates the $\mathrm{SO}_{2}$ dips in bags $\mathrm{D}, \mathrm{G}$ and $\mathrm{H}$, after day 30,36 and 39 respectively. Although all bags eventually drop in $\mathrm{SO}_{2}$ value, they normally do so after 50-60 days. These uncharacteristic drops in $\mathrm{SO}_{2}$ values caused the bags to visually turn from red to black in between two consecutive experimental days.

Upon discussions with CBS lab technicians, it is speculated that the sharp drops in $\mathrm{SO}_{2}$ are due to some form of contamination. One reason may be that the couplers used for entry into the blood bags for thesis experiments have undergone above-average needle penetration and therefore increased the probability of contamination. According to the lab technicians at CBS, needle insertion for blood extraction averages around 20-30 times per bag for their work; taking into account all thesis experiments and lab use, the needle insertion average for bags A-H was in the range of 80-100. The possibly degraded rubber stopper of the coupler was, with the rest of the 
bag, immersed 15-20 times in water and was put under pressure from the container setup (Section 2.7.4). These factors could have contributed to an increased probability of contamination.

One of the key adjustments to the experimental protocol for reduction of contamination would be to seal the coupler with parafilm or any other protecting element that can shield the entry of the coupler to the water environment during experiments.
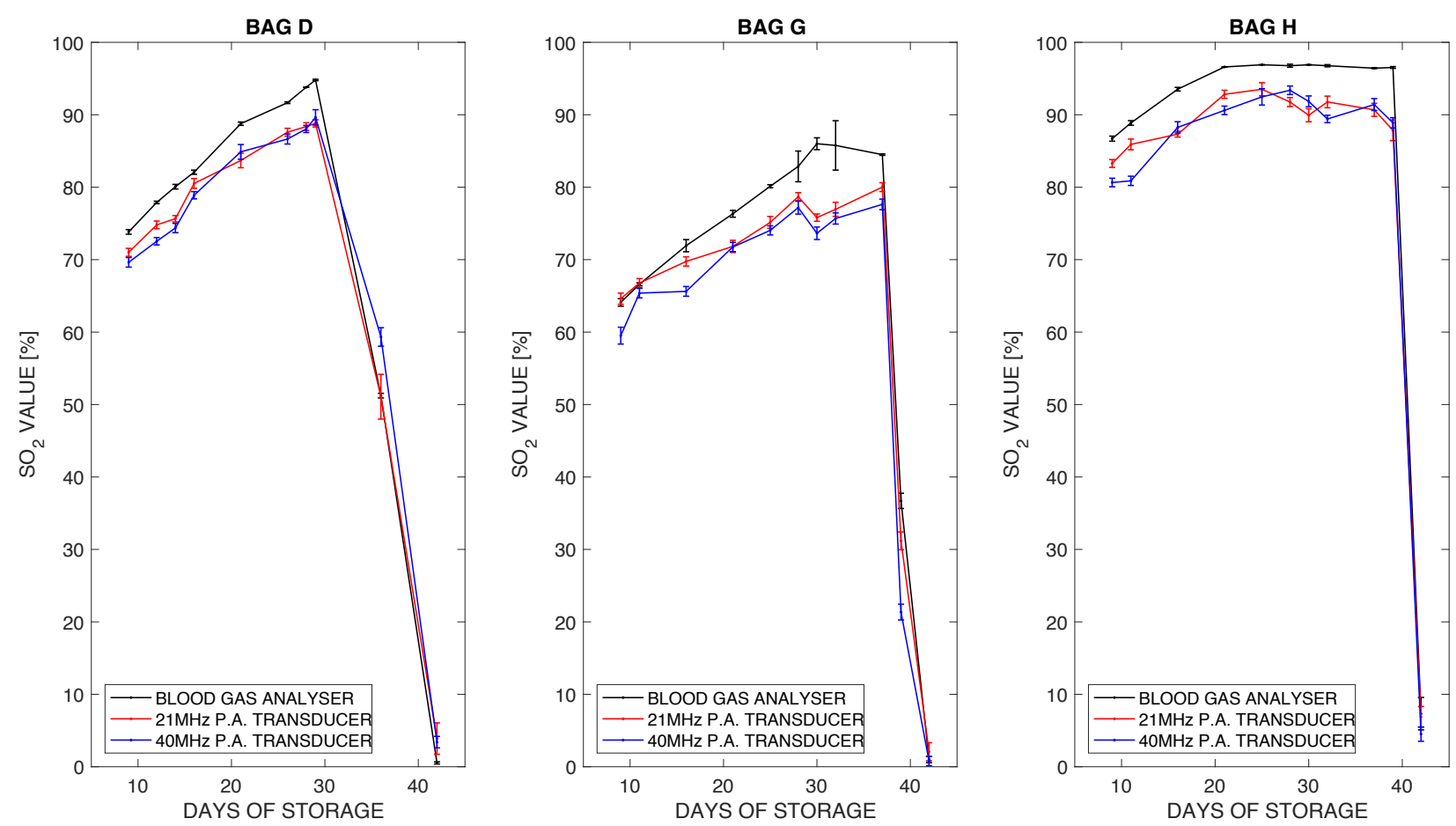

Figure 4-4: Abrupt declines in FLEX and LAZR in situ $\mathrm{SO}_{2}$ values for bags $\mathrm{D}, \mathrm{G}$ and $\mathrm{H}$ 


\section{Chapter 5 : Future Work}

\subsection{Validating the RBC-MIST}

The error associated with incorrectly segregating IFC images in the RBC-MIST was quantitatively assessed during development by observing image populations produced during each step (Section 3.2). This error was not quantified, nor was the template validated through any other external method. A natural step towards gaining an understanding of the capabilities and limitations of the RBC-MIST lies in comparing it against the conventional methods of RBC morphology characterization.

As introduced in Section 1.7.1, the MI method is the current gold standard assay adopted by CBS and other blood bank agencies to assess the morphology of a sample of RBCs. Since the conventional method measures RBC subpopulations using the MI, this technique could be translated towards an expanded, modified version of the RBC-MIST technique. While the standard assay multiplies the MI scale factors against the number of images (out of 100) observed for each subpopulation (Table 1-3), the IFC method would perform the multiplication with the relative percentages for each subpopulation. Hence, further work should be focused on further segmenting the subpopulations currently acquired by the RBC-MIST (Figure 3-4); this includes primarily the segregation of smooth discs from crenated discs and crenated spheroids from crenated spheres.

Some preliminary techniques for the segmentation of RBC samples into subpopulations are presented in Table 5-1. Future work will be concentrated on the development of customized masks to measure the minute details differentiating the various $\mathrm{RBC}$ subpopulations. If segmentation can be accomplished, an IFC/light microscopy validation study can be established. The experiments should involve simultaneous (within 24 hours) IFC and light microscopy measurements of RCC samples from the same blood bag. The MI values attained from both 


\begin{tabular}{|c|c|c|c|c|c|c|}
\hline DISCRIMINATING SUBPOPULATION & \multicolumn{2}{|c|}{ SAMPLE IMAGES } & KEY DISCRIMINATING PARAMETER & \multicolumn{2}{|c|}{ SAMPLE IMAGES } & DISCRIMINATING SUBPOPULATION \\
\hline SMOOTH DISCS: Larger Circularity & 80311 & 79862 & $\begin{array}{l}\text { CIRCULARITY: Degree of mask } \\
\text { deviation from a perfect circle. } \\
\text { Calculated by dividing the average } \\
\text { distance of boundary from its center } \\
\text { by the variation of the distance }\end{array}$ & 7664 & & CRENATED DISCS: Smaller Circularity \\
\hline CRENATED DISCS: Smaller Spot Count & 7664 & 355 & $\begin{array}{l}\text { SPOT COUNTS: The number of bright, } \\
\text { mutually exclusive mask spots after } \\
\text { eroding the image mask. }\end{array}$ & 67019 & 341 & $\begin{array}{l}\text { CRENATED DISCOIDS: Larger Spot } \\
\text { Count }\end{array}$ \\
\hline CRENATED DISCOIDS: Larger Diameter & 67015 & 341 & $\begin{array}{l}\text { DIAMETER: The diameter of a circle } \\
\text { with same aread as mask. }\end{array}$ & 3755 & 986 & $\begin{array}{l}\text { CRENATEDS SPHEROIDS: Smaller } \\
\text { Diameter }\end{array}$ \\
\hline $\begin{array}{l}\text { CRENATED SPHEROIDS: Higher H } \\
\text { Texture Average }\end{array}$ & 3755 & 9 & $\begin{array}{l}\text { H TEXTURE: A measure of the intensity } \\
\text { distribution and spatial relationship of } \\
\text { pixels within mask. }\end{array}$ & 6008 & 8226 & $\begin{array}{c}\text { CRENATED SPHERES: Lower H Texture } \\
\text { Average }\end{array}$ \\
\hline
\end{tabular}

Table 5-1: General features suggested for segregating pairs of subpopulations provided in each row. Sample images display masked cells. Circularity, spot counts and diameter texture features details provided in [207]; $\mathrm{H}$ textural features published by Haralick et al. [217].

methods could also be compared across the lifespan of the RCC. Ideally, the conventional MI technique should be performed by CBS lab technicians to eliminate any biasing. Such an experiment would allow for a quantitative measure of the ability of the RBC-MIST to accurately segregate RBC images into subpopulations.

Furthermore, if the RBC-MIST is validated, it could be used as a powerful tool to study the effect of donor variability (age, sex etc.) and manufacturing methods on the RCC morphology distribution. The IFC assay could potentially serve as a novel data set to complement the current quality control data collected by CBS to study sources of RCC variability [194].

\subsection{Validating PA Measurements of Blood Bags in situ}

$\mathrm{SO}_{2}$ values acquired from blood bags in situ using the PA apparatus were shown to have strong correlation with gold standard $\mathrm{BGA} \quad \mathrm{SO}_{2}$ measurements, however, were also found to be consistently lower (Sections 3.4, 4.4) The discrepancy in values was suggested to be due to an increase in $\mathrm{SO}_{2}$ of samples extracted from the bag for FLEX measurements (Figures 4.2, 4.3). Ideally, a validation of in situ measurements of $\mathrm{SO}_{2}$ should be accomplished with another in situ $\mathrm{SO}_{2}$ technique. The optical technique of diffuse reflectance (DR) provides potential for such a 
comparison, primarily due to its ability to non-invasively acquire $\mathrm{SO}_{2}$ using the same optical window as PA.

Preliminary work on the setup for the experiment involved one fiber optic probe that was placed on the surface of the blood bag to illuminate and receive signal. The probe was connected to a spectrometer which was associated with a software program that displayed signal in real time. In order to produce an unsaturated signal, the probe was held at an angle and the blood bags were flattened at the ROI. The bags were placed on a black background in a dark environment to reduce any ambient signal/reflection. For the reference, a blood bag containing the CPS/SAGM additive solution was measured; this signal, as well was a background measurement (no incident light), was subtracted from the blood bag signal to produce the collected reflectance spectra. A proprietary algorithm provided by the program converted the reflectance spectra into absorbance.

Figure 5.1 displays the absorption spectra of three trials in the $600 \mathrm{~nm}-900 \mathrm{~nm}$ range for three blood bags (A, B and D). A comparison against hemoglobin spectra Figure 1-17 clearly indicates that bags $\mathrm{A}$ and $\mathrm{B}$ were oxygenated, while $\mathrm{D}$ was deoxygenated. As a consequence of holding the probes by hand, spectra tended to vary significantly; an example of this variation is captured with the data from bag D. Hence, future work should focus on the obtaining a hands free setup for both blood bag and probe placement. Such a setup will allow for optimizing parameters such as probe-bag distances and angles for increasing signal strength. Once a suitable setup is developed, a study can be carried out to acquire $\mathrm{SO}_{2}$ of blood bags in situ using published techniques on diffuse reflectance $\mathrm{SO}_{2}$ calculations [195]. 

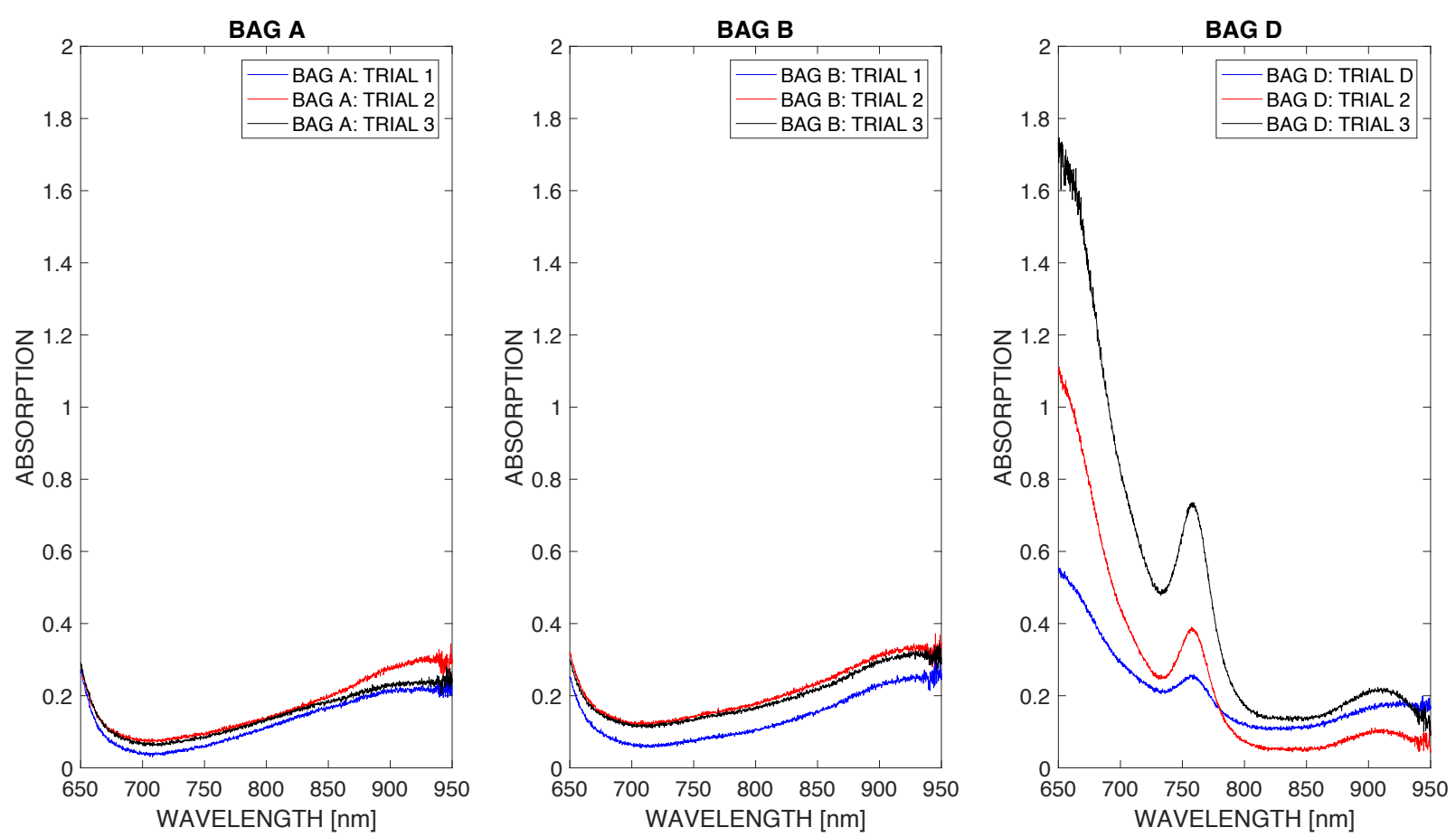

Figure 5-1: Derived absorption spectra acquired from DR measurements of three blood bags in situ. The spectral behavior in this region provides an indication of the oxygenation of the blood bags.

\subsection{Radio-Frequency Analysis of PA Signals}

The PA radio-frequency (RF) signals received by the LAZR, when converted into the frequency domain, can theoretically derive multiple parameters that are characteristic of the sizes and shapes of the PA absorbers [182]. The potential for PA to access both $\mathrm{SO}_{2}$ and morphological information of RBCs in situ gives the technology a significant advantage over other optical techniques. Future work will investigate the potential of PA RF spectroscopic analysis of blood bags in situ, to determine if changes in spectral parameters can be measured over the lifespan of the blood bags. In a manner very similar to the MI method, PA RF spectroscopy could potentially provide morphologically-related parameters that can be monitored for change over time. These changes could be evaluated alongside MI changes to establish a correlation. If a strong correlation exists, as is the case with in situ $\mathrm{PA} \mathrm{SO}_{2}$ values and the gold standard method, PA technology would show a potential of non-invasively monitoring both functional and morphological parameters of RBCs stored in blood bags. 


\section{Chapter 6: Conclusions}

Results acquired from the IFC and BGA instruments over the lifespan of seven blood bags support the hypothesis stating that there is a correlation between spheroechinocyte percent change and the $\mathrm{RBC} \mathrm{SO}_{2}$ percent change during the in vitro storage of a blood bag. Excluding the outlier - blood bag $\mathrm{H}$ - the spheroechinocyte $/ \mathrm{SO}_{2}$ correlation was found to be strong $\left(0.79<\mathrm{r}^{2}<0.91\right)$. A novel finding from this work was the discovery of similar percent increases (over time) in $\mathrm{SO}_{2}$ and spheroechinocyte population. This agreement between the two parameters suggests that by tracking $\mathrm{SO}_{2}$ increase over time, an approximation of the increase in spheroechinocyte percentage could be made.

Experimental data has supported the hypothesis that PA can monitor the $\mathrm{SO}_{2}$ of $\mathrm{RBCs}$ in blood bags in situ. $\mathrm{r}^{2}$ values between $\mathrm{BGA}$ and $\mathrm{PA} \mathrm{SO}_{2}$ values across the lifespan of seven blood bags were found to be very strong, exceeding 0.95 for both the $21 \mathrm{MHz}$ and $40 \mathrm{MHz}$ transducers. However, Bland-Altman plots showed that $\mathrm{BGA} \mathrm{SO}_{2}$ values were approximately $4 \%$ higher than those acquired by PA. Experimental data has supported the conjecture that BGA values were consistently higher due to unavoidable contact with air during the extraction of RBCs. The results suggested that there is strong potential for PA to accurately measure the $\mathrm{SO}_{2}$ of $\mathrm{RBCs}$ in blood bags in situ.

Since the $\mathrm{SO}_{2}$ increase is similar in value to the spheroechinocyte percentage increase of RBCs stored in vitro, and since non-invasive $\mathrm{PA} \mathrm{SO}_{2}$ values have a very strong correlation with BGA $\mathrm{SO}_{2}$ values, an indirect link between PA and IFC data can be made. Hence, PA has the potential to provide information on functional changes non-invasively, while simultaneously inferring the changes in morphological distribution. PA could be the first optical technology to monitor certain measures of blood bag quality without compromising the bags for transfusion.

Future work will focus on robustly validating of the novel techniques of the IFC RBC-MIST and the in situ $\mathrm{SO}_{2}$ measurements of blood bags via PA. The frequency content of PA will be investigated to determine if time-dependent morphological information can be extracted. 


\section{Appendices}

\section{Appendix A: Supplementary Tables and Figures}

\begin{tabular}{|l|l|}
\hline Specifications & SafePICO Aspirator \\
\hline Mixing device & $3.5 \mathrm{~mm}$ mixing ball \\
\hline Heparin & $\begin{array}{l}\text { 80 IU electrolyte-balanced heparin, coated on } \\
\text { cellulose fiber }\end{array}$ \\
\hline Sample volume & $\begin{array}{l}0.7-1.7 \mathrm{~mL} \text {. A sample volume above } 1 \mathrm{~mL} \text { is } \\
\text { required when measuring ctHb }\end{array}$ \\
\hline Sterilization & By ethylene oxide \\
\hline Unique sampler ID & Code 128 barcode \\
\hline
\end{tabular}

Table A-1: List of some specification of the safePICO Aspirator syringe provided by Radiometer [200].

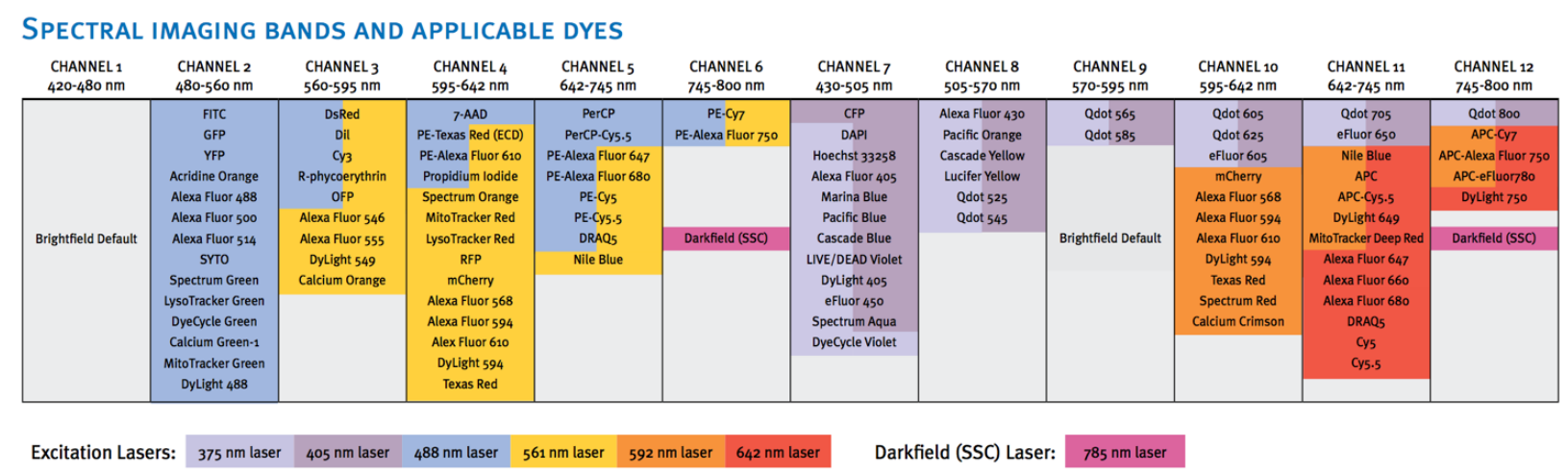

Table A-2: Spectral Features of IS $^{\mathrm{X}}$, including channel bandwidths, applicable dyes for available lasers. Not included is $830 \mathrm{~nm}$ laser used to scatter light from beads in sheath flow for detection in autofocus/flow speed system. Adapted from [204]. 


\begin{tabular}{|c|c|c|}
\hline MASK NAME & BRIEF DESCRIPTION & PARAMETERS FOR CUSTOMIZING \\
\hline Inspire & the mask used during data acquisition in INSPIRE & - \\
\hline Dilate & Adds the selected number of pixels to all edges of the default mask & Pixel Number \\
\hline Erode & Removes the selected number of pixels from all edges of the default mask & Pixel Number \\
\hline Inspire & Fills any holes in the default mask & - \\
\hline Intensity & Masks pixels between the lower and upper raw intensity thresholds & Threshold Value \\
\hline \multirow[t]{2}{*}{ Interface } & Identifies pixels in an object where the object is in contact with a second object & Mask to cover the object of interest \\
\hline & & Mast to cover both objects \\
\hline Morphology & Includes all pixels within the outermost image contour of fluorescence images & - \\
\hline Object & Segments images to closely identify the area corresponding to the cell & - \\
\hline Peak & identifies intensity areas from an image that have local maxima (bright) or minima (dark) & - \\
\hline \multirow[t]{2}{*}{ Range } & select components in an image within a selected size and/or aspect ratio & Min/Max Area \\
\hline & & Min/Max Aspect Ratio \\
\hline \multirow[t]{2}{*}{ Skeleton } & Provides the barebone structure of the object from the starting mask & Option of "thin" skeleton (1 pixel wide) \\
\hline & & Or "thick" skeleton (Intensity Weighted) \\
\hline Spot & Obtains bright or dark regions from an image regardless of the intensity & Extent of Image Erosion \\
\hline System & Segments objects in an image based on a probability model & "Weight" Value for Grouping \\
\hline Threshold & Excludes pixels, based on a intensity ranges of default mask. & - \\
\hline Valley & A rectangular mask that is created between two bright regions in a default mask & - \\
\hline
\end{tabular}

Table A-3: Names, brief descriptions and parameters of the masks available on IDEAS ${ }^{\circledR}$ [207]. 
SIZE

LOCATION

AREA : Size of mask in square microns

DIAMETER: Estimates diameter of mask based on circle of same area

HEIGHT: The longest dimension of a bounding rectangle

WIDTH: The shortest dimension of a bounding rectangle

LENGTH: Longest part of the mask

MAJOR/MINOR AXIS: Widest and nar rowest part of the mask respectively

MAJOR/MINOR AXIS INTENSITY: The nar rowest and widest part, respectively, of a binding ellipse

PERIMETER: Circumference of the mask

SPOT AREA: Area of brightest or darkest mask spot after eroding mask

ANGLE: Angle of major ax is from horizontal plane in radians

CENTROID X \& Y: Central tendency of pixels along $x$ and $y$-ax is respectively

DELTA CENTROID $X / Y$ : Distance between $X$ or $Y$ centroid, respectively, of two images

DELTA CENTROID XY: Average distance between the cent roid of two images

MAX CONTOUR POSITION: Location of cell contour producing highest intensity concent ration

SHAPE

TEXTURE

ASPECT RATIO: Ratio of minor axis over major axis

CIRCULARITY: Ratio of av era ge distance of boundary from center over va riation in dist ances

COMPACTNESS: Density of intensities within mask

ELONGATEDNESS: Ratio of height over width of bounding box

SYMMETRY 2: Tendency of mask to have single axis of elongation, and hence 2 lobes within a mask

CONTRAST: Measures sharpness quality of image by detecting large changes in pixel values

GRADIENT MAX: Maximum gradient of pixel value changes within mask

GRA DIENT RMS: Root mean square of gradient values between pixels in maskto measure image focus

H TEXTURE: Measures Harlick Text ural features [217]

MODULATION: Intesity range of an image normalized between 0 and 1

SPOT COUNT: Number of bright or dark mask spots aft er eroding mask

STD DEV: Overall distribution of pixel intensities

SIGNAL STRENGTH

BKGD MEAN: Average intensity of ca mera background

BKGD StdDev: Standard Deviation of camera background intensities

INTENSITY: sum of pixel int ensities in a mask, bac kground subt racted

MAX/MIN PIXEL: Maximum and minimum, respectively, pixel value within a mask, back ground subt rac ted

MEAN/MEDIAN PIXEL: Mean and Median, respectively, of pixel values in mask, backg round subtracted

RAW INTENSITY: sum of pixel intensities in a mask

RAW MAX/MIN/MEAN/MEDIAN PIXEL: intensities of pixels are not background subtracted

COMPARISON

SATURATION COUNT/PERCENT: Number and percent, respectively, of mask pixels that are saturated

BRIGHT DETAIL SIMILARITY: Measures correlation of bright details between pairs of images INTENSITY CONCENTRATION RATIO: Ra tio of intensity of one mask over the total int ensity of both masks INTERNALIZATION: Ratio of the intensityinside the cell over the intensity of the entire cell SIMILARITY: Degree to which two images a re correlated for each pixel within the mask

SYSTEM

CAMERA LINE NUMBER: Incremental count of objects

CAMERATIMER: Clock rate in $\mathrm{KHz}$

FLOW SPEED: Flow speed in $\mathrm{mm} / \mathrm{s}$

OBJECT NUMBER: Sequence of objects

OBJECT/ml: Number of objects per $\mathrm{ml}$

OBJECTS/sec: Number of objects per second

TIME: Camera timer converted to seconds

Table A-4: Names and brief descriptions of the features available on IDEAS $^{\circledR}$ [207]. 


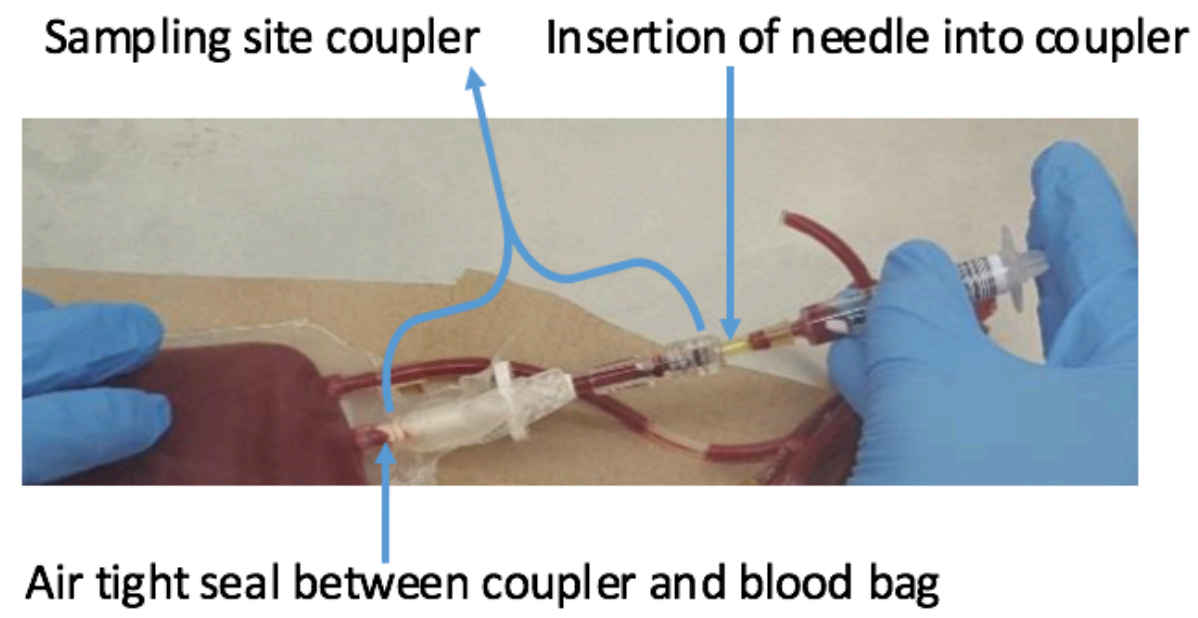

Figure A-1: Picture of a blood bag fitted with a sampling site coupler, with a needle inserted into the coupler for RCC extraction.

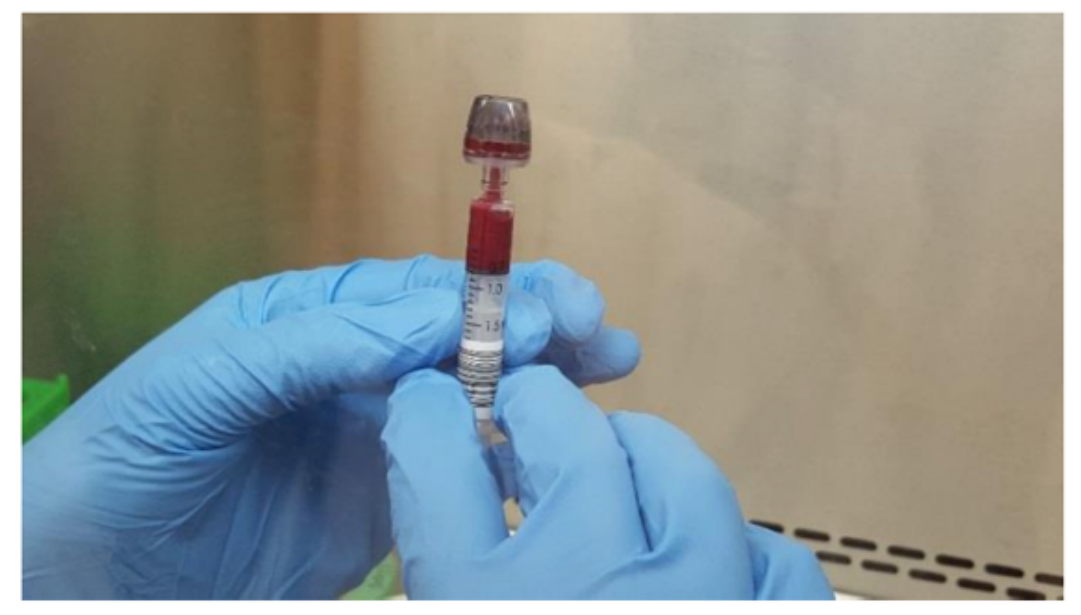

Figure A-2: Picture displaying the safeTIPCAP driving air out of the sample while sealing the sample within the syringe.

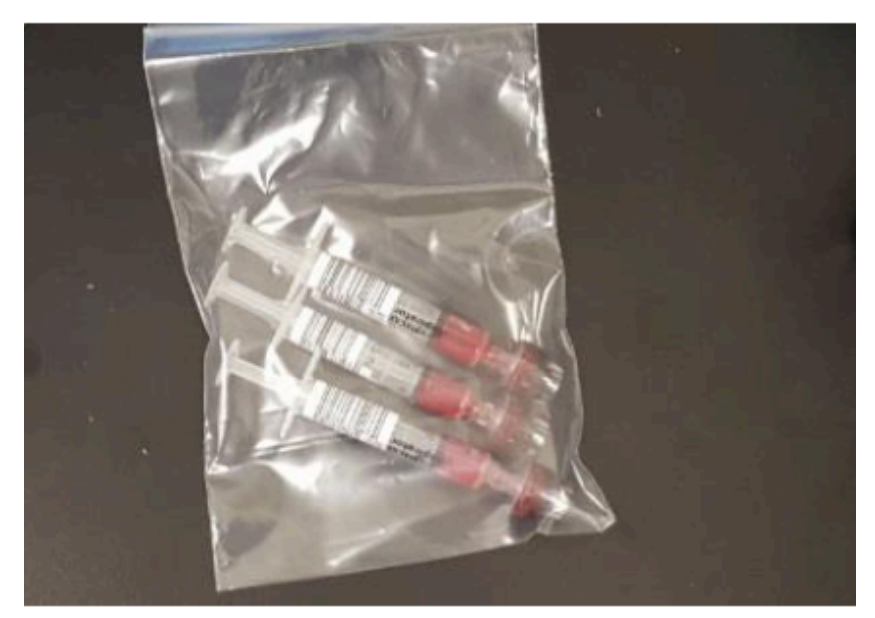

Figure A-3: Picture of three samples extracted from one blood bag, ready for transport to FLEX. 


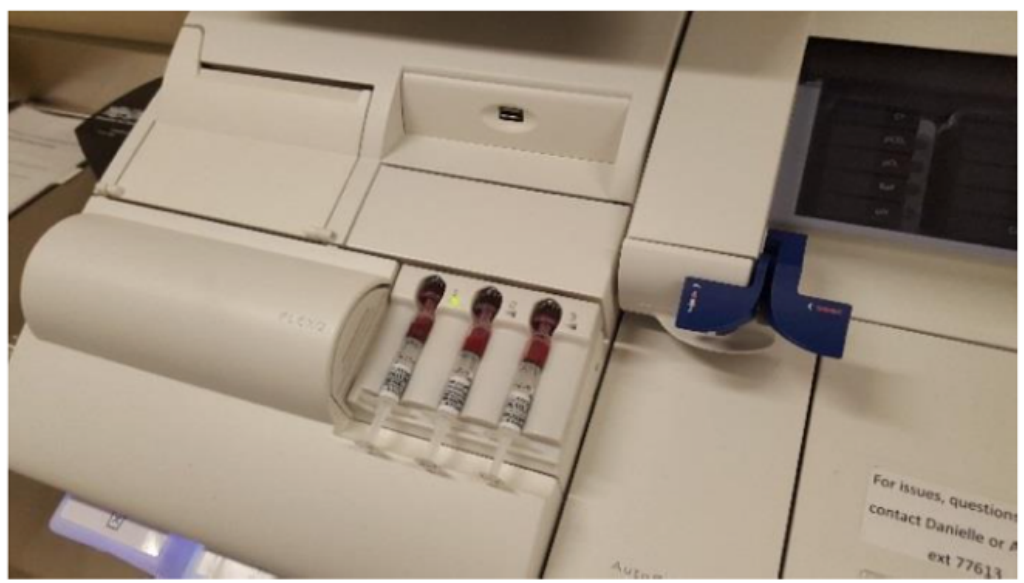

Figure A-4: Picture of three samples placed in the FLEX device for automated analysis.

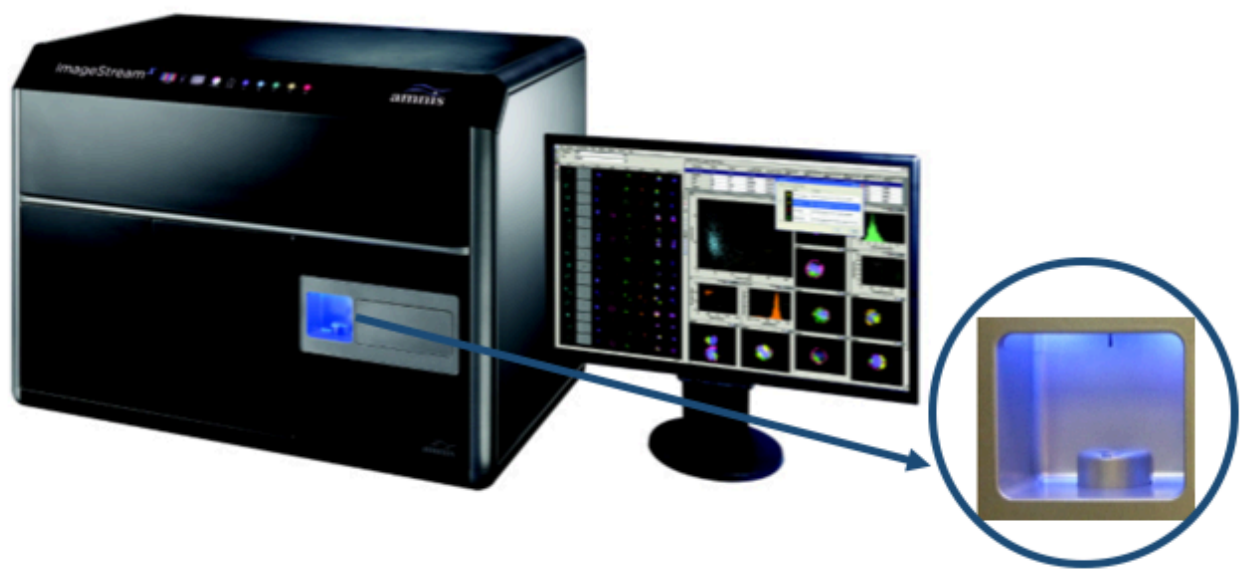

Figure A-5: Picture of (left) the IS ${ }^{\mathrm{X}}$ device, as well as (right) the sample holder area for vial placement. Images adapted from [205].

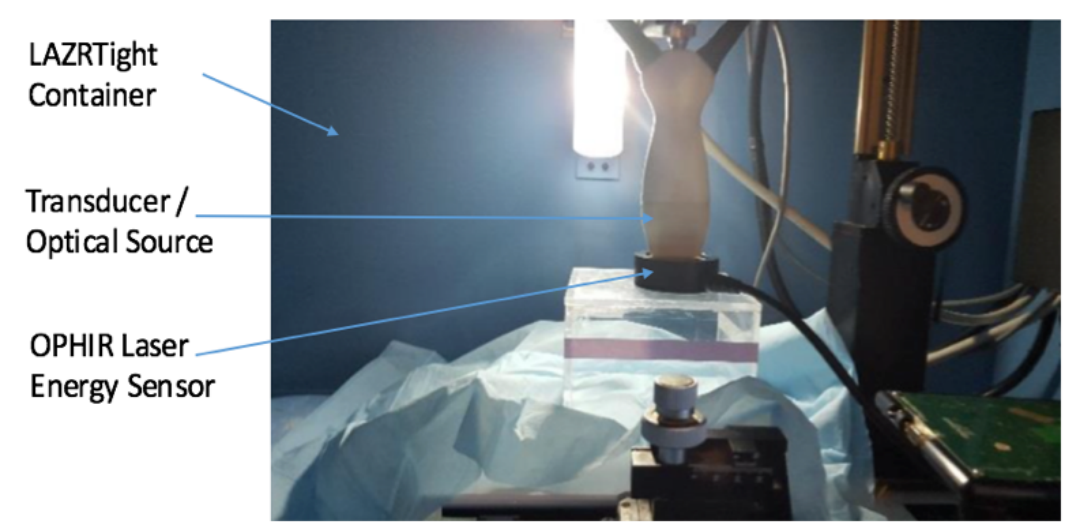

Figure A-6: Setup of the LAZR laser calibration, to correct for differences between the LAZR cart energy values and the transducer output energy values. 


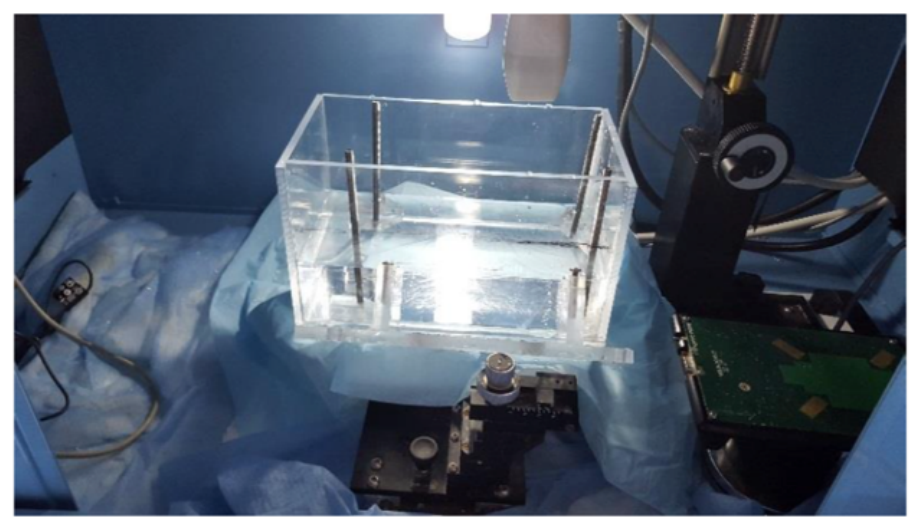

Figure A-7: Positioning of blood bag LAZR container within the LAZRTight container.
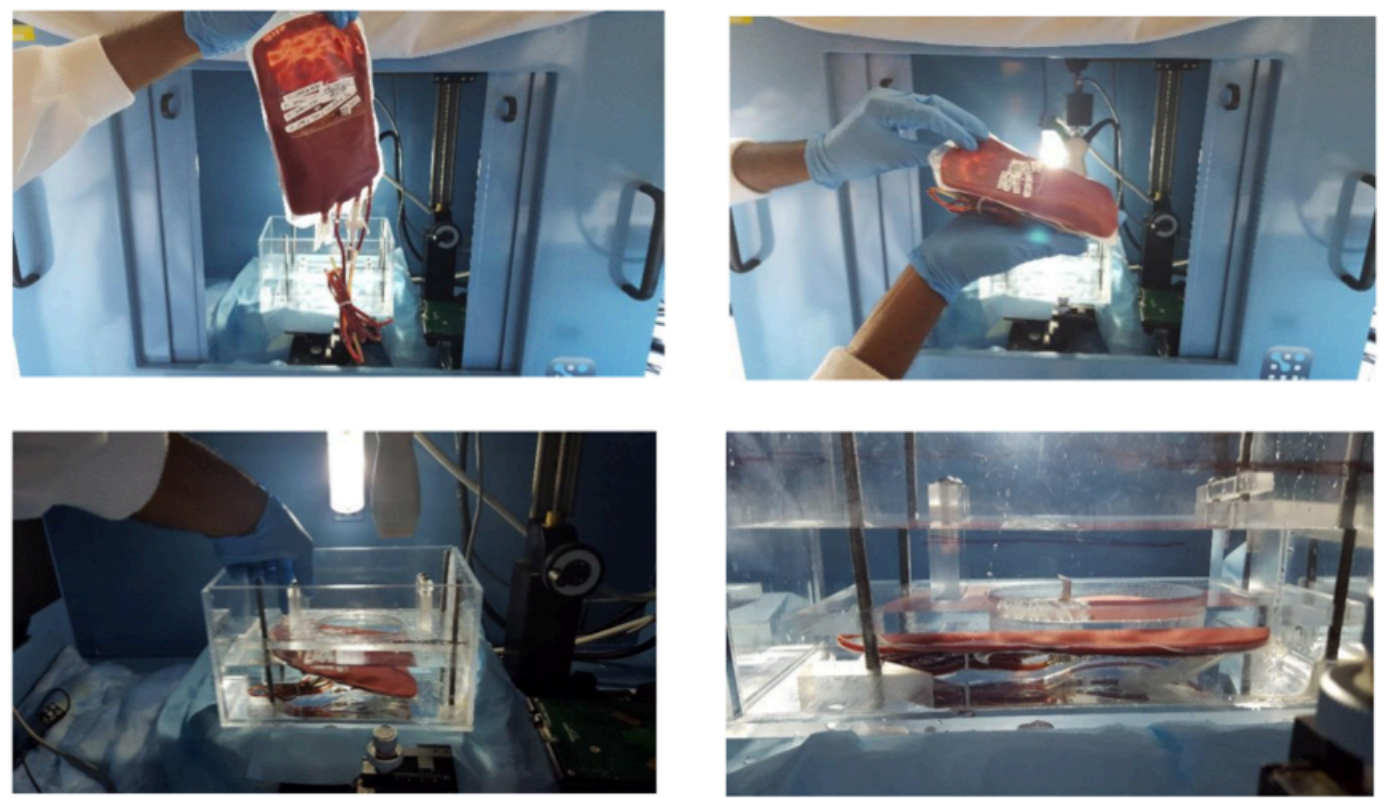

Figure A-8: blood bag LAZR setup: (top left) hanging of bag to separate air pockets; (top right) folding of coupler and tubing; (bottom left) gradual angled sliding of bag onto the container bed and; (bottom right) simultaneous introduction of container lid for horizontal flattening and stability of bag.
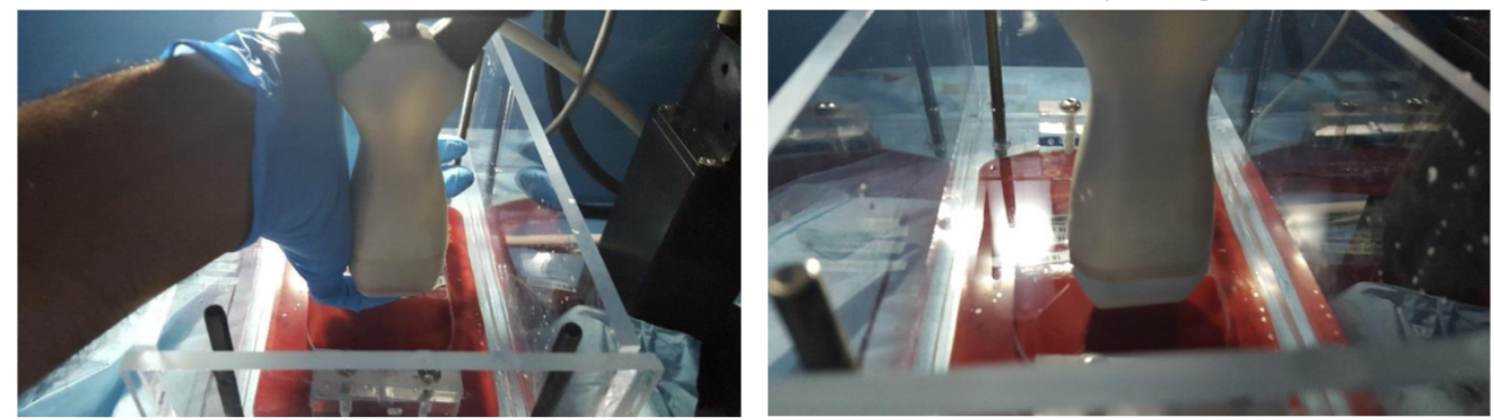

Figure A-9: (left) removing of air bubbles after insertion into water and (right) final position of transducer over blood bag for $\mathrm{PA} \mathrm{SO}_{2}$ measurements. 


\section{Appendix B: Derivation of Absorbance and Molar Extinction Coefficient}

Figure B-10 illustrates an incident monoenergetic beam of photons of intensity $\mathrm{I}_{0}$ traversing through a slab of thickness $\mathrm{d} x$, after which the intensity decreases by an amount dI due to absorbing species in the slab. A linear absorption coefficient $\left(\mu_{\mathrm{a}}\right)$ is assigned to characterize the species causing the absorption. The absorption coefficient is a product of the effective absorption cross sectional area $\left(\sigma_{\mathrm{a}}\right)$ and the volumetric density $(\mathrm{C})$ of the species:

$$
\mu_{\mathrm{a}}\left[\mathrm{cm}^{-1}\right]=\sigma_{\mathrm{a}}\left[\mathrm{cm}^{2}\right] \times C\left[\# \text { molecules } \cdot \mathrm{cm}^{-3}\right]
$$

Furthermore, the Absorbance (A) and Transmittance $(\mathrm{T})$ across the entire slab is defined as:

$$
\mathrm{A}=-\log (T), \quad \mathrm{T}=\frac{\mathrm{I}}{\mathrm{I}_{0}}
$$

Using the linear absorption coefficient as the constant of proportionality, the rate of change in intensity with respect to the thickness of the slab can be expressed as:

$$
\frac{\mathrm{dI}}{\mathrm{d} x}=-\mu_{\mathrm{a}} \mathrm{I}
$$

Rearranging gives:

$$
\frac{\mathrm{dI}}{\mathrm{I}}=-\mu_{\mathrm{a}} \mathrm{d} x
$$

Assigning the incident intensity as $\mathrm{I}_{0}$ and integrating over the limits provided in Figure B-10 gives:

$$
\int_{\mathrm{I}_{0}}^{\mathrm{I}} \frac{\mathrm{dI}}{\mathrm{I}}=-\mu_{\mathrm{a}} \int_{0}^{x} \mathrm{~d} x \Rightarrow \ln (\mathrm{I})-\ln \left(\mathrm{I}_{0}\right)=-\mu_{\mathrm{a}} x \Rightarrow \ln \left(\frac{\mathrm{I}}{\mathrm{I}_{0}}\right)=-\mu_{\mathrm{a}} x
$$

Substituting the relations provided by Equation B2 into the left hand side of the last expression in Equation B5, the following relation is established between absorbance and transmittance:

$$
-\ln (\mathrm{T})=\log _{\mathrm{e}}\left(\frac{1}{\mathrm{~T}}\right)=\frac{\log _{10}\left(\frac{1}{\mathrm{~T}}\right)}{\log _{10} \mathrm{e}}=\frac{\log _{10}\left(\frac{1}{\mathrm{~T}}\right)}{0.434}=\mathrm{A}
$$

Substituting B1 and B6 into the last expression of equation B5 gives:

$$
\mathrm{A}=\log _{10}\left(\frac{1}{\mathrm{~T}}\right)=0.434 \cdot \sigma \cdot \mathrm{C} \cdot x
$$

which contains the definition of the extinction coefficient:

$$
\varepsilon=0.434 \cdot \sigma
$$


$\varepsilon$ can be converted into the molar extinction coefficient when multiplied with Avogadro's number:

$$
\varepsilon=0.434 \cdot\left(\frac{6.02 \times 10^{23}}{\mathrm{~mol}}\right) \cdot \sigma
$$

It is more convenient to express the concentration of absorbing species such as RBCs in molar rather than volumetric units, hence the units can be converted as follows:

$$
\varepsilon \cdot C \cdot x \Leftrightarrow\left[\frac{\mathrm{cm}^{2}}{\mathrm{~mol}}\right] \cdot\left[\frac{\mathrm{mol}}{\mathrm{cm}^{3}}\right] \cdot[\mathrm{cm}] \stackrel{1000 \mathrm{~cm}^{3} \equiv 1 \mathrm{~L}}{=}\left[\frac{\mathrm{L}}{\mathrm{mol} \cdot \mathrm{cm}}\right] \cdot\left[\frac{\mathrm{mol}}{\mathrm{L}}\right] \cdot[\mathrm{cm}]
$$

Therefore, the units of the molar extinction coefficient can be expressed as $\mathrm{M}^{-1} \cdot \mathrm{cm}^{-1}$

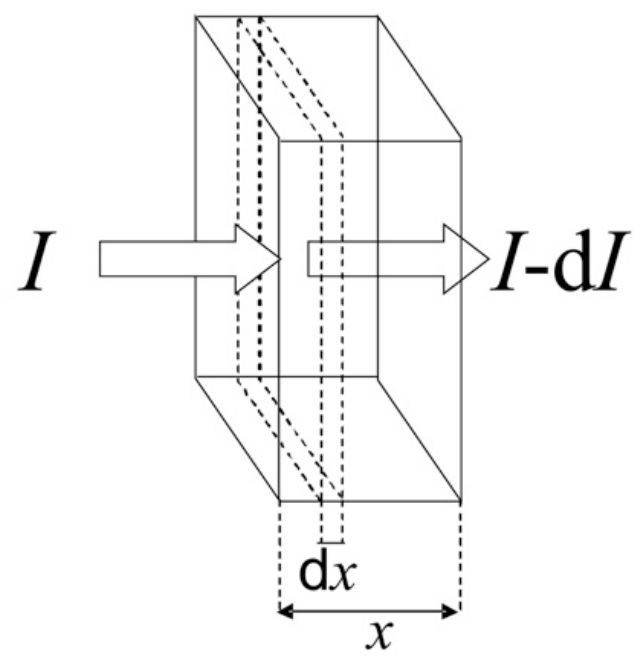

Figure B-10: Schematic representation outlining the key variables used in the derivation of Beer's law. Photons of intensity $I_{0}$ traverse through a slab of thickness $d x$, after which the intensity decreases by an amount $d I$ due to absorbing/scattering species in the slab. Adapted from [196]. 


\section{Appendix C: Derivation of Oximetry Equation for $\mathrm{SO}_{2}$}

Assuming that the only states of hemoglobin are oxyHb and deoxyHb, Equation B6 can be expressed for two equations in order to solve for the two unknown concentration of hemoglobin species:

$$
\begin{aligned}
& A_{\text {tot }}^{\lambda_{1}}=\left(\varepsilon_{\mathrm{HbO}_{2}}^{\lambda_{1}} C_{\mathrm{HbO}_{2}}+\varepsilon_{\mathrm{Hb}}^{\lambda_{1}} C_{\mathrm{Hb}}\right) \cdot x \\
& A_{\text {tot }}^{\lambda_{2}}=\left(\varepsilon_{\mathrm{HbO}_{2}}^{\lambda_{2}} \mathrm{C}_{\mathrm{HbO}_{2}}+\varepsilon_{\mathrm{Hb}}^{\lambda_{2}} C_{\mathrm{Hb}}\right) \cdot x
\end{aligned}
$$

where $A_{\text {tot }}$ represents the measured absorbance at each wavelength, $\varepsilon$ represents the known tabulated molar extinction coefficient for each hemoglobin state and wavelength, $C$ is the concentration of each hemoglobin state and $\mathrm{x}$ is the pathlength of light. The oxygen saturation equation is expressed as:

$$
\mathrm{SO}_{2}=\frac{\mathrm{C}_{\mathrm{HbO}_{2}}}{\mathrm{C}_{\mathrm{HbO}_{2}}+\mathrm{C}_{\mathrm{Hb}}}
$$

Subtracting $\mathrm{SO}_{2}$ from 1 gives:

$$
1-\mathrm{SO}_{2}=1-\frac{\mathrm{C}_{\mathrm{HbO}_{2}}}{\mathrm{C}_{\mathrm{HbO}_{2}}+\mathrm{C}_{\mathrm{Hb}}}=\frac{\mathrm{C}_{\mathrm{Hb}}}{\mathrm{C}_{\mathrm{HbO}_{2}}+\mathrm{C}_{\mathrm{Hb}}}
$$

Isolating the numerators of Equation $\mathrm{C} 3$ and Equation $\mathrm{C} 4$ gives:

$$
\begin{gathered}
\mathrm{C}_{\mathrm{HbO}_{2}}=\mathrm{SO}_{2} \cdot\left(\mathrm{C}_{\mathrm{HbO}_{2}}+\mathrm{C}_{\mathrm{Hb}}\right) \\
\mathrm{C}_{\mathrm{Hb}}=\left(1-\mathrm{SO}_{2}\right) \cdot\left(\mathrm{C}_{\mathrm{HbO}_{2}}+\mathrm{C}_{\mathrm{Hb}}\right)
\end{gathered}
$$

Substituting Equation C5 and Equation C6 into Equation C1 and Equation C2:

$$
\begin{aligned}
& \mathrm{A}_{\mathrm{tot}}^{\lambda_{1}}=\left(\varepsilon_{\mathrm{HbO}_{2}}^{\lambda_{1}} \mathrm{SO}_{2}+\varepsilon_{\mathrm{Hb}}^{\lambda_{1}}\left(1-\mathrm{SO}_{2}\right)\right) \cdot\left(\mathrm{C}_{\mathrm{HbO}_{2}}+\mathrm{C}_{\mathrm{Hb}}\right) \cdot x \\
& \mathrm{~A}_{\mathrm{tot}}^{\lambda_{2}}=\left(\varepsilon_{\mathrm{HbO}_{2}}^{\lambda_{2}} \mathrm{SO}_{2}+\varepsilon_{\mathrm{Hb}}^{\lambda_{2}}\left(1-\mathrm{SO}_{2}\right)\right) \cdot\left(\mathrm{C}_{\mathrm{HbO}_{2}}+\mathrm{C}_{\mathrm{Hb}}\right) \cdot x
\end{aligned}
$$

Rearranging terms in Equation C7 and Equation C8:

$$
\begin{aligned}
& \mathrm{A}_{\text {tot }}^{\lambda_{1}}=\left(\mathrm{SO}_{2}\left[\varepsilon_{\mathrm{HbO}_{2}}^{\lambda_{1}}-\varepsilon_{\mathrm{Hb}}^{\lambda_{1}}\right]+\varepsilon_{\mathrm{Hb}}^{\lambda_{1}}\right) \cdot\left(\mathrm{C}_{\mathrm{HbO}_{2}}+\mathrm{C}_{\mathrm{Hb}}\right) \cdot x \\
& \mathrm{~A}_{\text {tot }}^{\lambda_{2}}=\left(\mathrm{SO}_{2}\left[\varepsilon_{\mathrm{HbO}_{2}}^{\lambda_{2}}-\varepsilon_{\mathrm{Hb}}^{\lambda_{2}}\right]+\varepsilon_{\mathrm{Hb}}^{\lambda_{2}}\right) \cdot\left(\mathrm{C}_{\mathrm{HbO}_{2}}+\mathrm{C}_{\mathrm{Hb}}\right) \cdot x
\end{aligned}
$$


For simplification, let:

$$
\Delta \varepsilon^{\lambda}=\left[\varepsilon_{\mathrm{HbO}_{2}}^{\lambda}-\varepsilon_{\mathrm{Hb}}^{\lambda}\right]
$$

Dividing Equation C9 by Equation C10 gives:

$$
\frac{\mathrm{A}_{\mathrm{tot}}^{\lambda_{1}}}{\mathrm{~A}_{\mathrm{tot}}^{\lambda_{2}}}=\frac{\mathrm{SO}_{2} \cdot \Delta \varepsilon^{\lambda_{1}}+\varepsilon_{\mathrm{Hb}}^{\lambda_{1}}}{\mathrm{SO}_{2} \cdot \Delta \varepsilon^{\lambda_{2}}+\varepsilon_{\mathrm{Hb}}^{\lambda_{2}}}
$$

Cross multiplication of the above equation leads to:

$$
\mathrm{A}_{\mathrm{tot}}^{\lambda_{1}} \cdot \mathrm{SO}_{2} \cdot \Delta \varepsilon^{\lambda_{2}}+\mathrm{A}_{\mathrm{tot}}^{\lambda_{1}} \cdot \varepsilon_{\mathrm{Hb}}^{\lambda_{2}}=\mathrm{A}_{\mathrm{tot}}^{\lambda_{2}} \cdot \mathrm{SO}_{2} \cdot \Delta \varepsilon^{\lambda_{1}}+\mathrm{A}_{\mathrm{tot}}^{\lambda_{2}} \cdot \varepsilon_{\mathrm{Hb}}^{\lambda_{1}}
$$

Grouping of the $\mathrm{SO}_{2}$ terms gives:

$$
\mathrm{A}_{\text {tot }}^{\lambda_{1}} \cdot \mathrm{SO}_{2} \cdot \Delta \varepsilon^{\lambda_{2}}-\mathrm{A}_{\mathrm{tot}}^{\lambda_{2}} \cdot \mathrm{SO}_{2} \cdot \Delta \varepsilon^{\lambda_{1}}=\mathrm{A}_{\mathrm{tot}}^{\lambda_{2}} \cdot \varepsilon_{\mathrm{Hb}}^{\lambda_{1}}-\mathrm{A}_{\mathrm{tot}}^{\lambda_{1}} \cdot \varepsilon_{\mathrm{Hb}}^{\lambda_{2}}
$$

Finally, isolating for $\mathrm{SO}_{2}$ results in the oximetry formula:

$$
\mathrm{SO}_{2}=\frac{\mathrm{A}_{\mathrm{tot}}^{\lambda_{2}} \cdot \varepsilon_{\mathrm{Hb}}^{\lambda_{1}}-\mathrm{A}_{\mathrm{tot}}^{\lambda_{1}} \cdot \varepsilon_{\mathrm{Hb}}^{\lambda_{2}}}{\mathrm{~A}_{\text {tot }}^{\lambda_{1}} \cdot \Delta \varepsilon^{\lambda_{2}}-\mathrm{A}_{\text {tot }}^{\lambda_{2}} \cdot \Delta \varepsilon^{\lambda_{1}}}
$$




\section{References}

[1] Maximow IV, A. A. (1924). Relation of blood cells to connective tissues and endothelium. Physiological Reviews, 4(4), 533-563.

[2] Callum, J. L., \& Pinkerton, P. H. (2015). Blood Transfusion: Information for Patients (2nd ed.). Chapter 1. ORBCON. Retrieved from http://inventory.transfusionontario.org/

[3] Jensen-Jarolim, E. (Ed.). (2013). Comparative medicine: Anatomy and physiology. Chapter 5.3.3. Retrieved from

http://ebookcentral.proquest.com.ezproxy.lib.ryerson.ca/lib/ryerson/detail.action?docID= 1592062

[4] Stefanovska, A. (1999). Physics of the human cardiovascular system. Contemporary Physics, 40(1), 31-55.

[5] Marieb, E. N., \& Hoehn, K. (2012). Human anatomy \& physiology ( $9^{\text {th }}$ Ed.). Chapter 17. Boston; Toronto: Pearson.

[6] Scott, K. L., Lecak, J., \& Acker, J. P. (2005). Biopreservation of red blood cells: past, present, and future. Transfusion medicine reviews, 19(2), 127-142.

[7] Mohandas, N., \& Chasis, J. A. (1993). Red blood cell deformability, membrane material properties and shape: Regulation by transmembrane, skeletal and cytosolic proteins and lipids. Seminars in hematology, 30(3), 171-192.

[8] Uzoigwe, C. "The human erythrocyte has developed the biconcave disc shape to optimise the flow properties of the blood in the large vessels." Medical hypotheses 67.5 (2006): 1159-1163.

[9] Canham, P. B., \& Burton, A.C. (1968). Distribution of size and shape in populations of normal human red cells. Circulation Research, 22(3), 405-422.

[10] Diez-Silva, M., et al. (2010). Shape and biomechanical characteristics of human red blood cells in health and disease. MRS bulletin, 35(5), 382-388.

[11] Kanias, T., \& Acker, J. P. (2010). Biopreservation of red blood cells - the struggle with hemoglobin oxidation. FEBS journal, 277(2), 343-356.

[12] A.D.A.M. Medical Encyclopedia. ImageID: 4482_72EWJ. Red Blood Cell and Hemoglobin. Atlanta GA. Retrieved from:

http://www.adamimages.com/Illustration/SearchResult/1/red\%20blood\%20cell

[13] Mozzarelli, A., Bruno, S., \& Ronda, L. (2013). Biochemistry of Hemoglobin. In

H. W. Kim \& A. G. Greenburg (Eds.). Hemoglobin-based oxygen carriers as red cell substitutes and oxygen therapeutics (55-73). Berlin; Heidelburg: Springer.

[14] Haymond, S. (2006). Oxygen Saturation: A Guide to Laboratory Assessment. Clinical Laboratory News, 10-12.

[15] Berg, J. M., Tymoczko, J. L. \& Stryer, L. (2002). BioChemistry (5 $5^{\text {th }}$ Ed.). Chapter 10.2. New York, NY: W.H. Freeman.

[16] Siggaard-Andersen, O., \& Garby, L. (1973). The Bohr effect and the Haldane effect. Scandinavian journal of clinical and laboratory investigation, 31(1), 1-8.

[17] Kelley, W. N. (2000). Kelley's textbook of internal medicine $\left(4^{\text {th }}\right.$ Ed.). Figure 241.2. Philadelphia, PA: Lippincott Williams \& Wilkins. Retrieved from www.optimedical.com/pdf/articles/oxygen-saturation-laboratory-assessment.pdf 
[18] Zock, J.P. (1987). Influence of 2, 3-DPG on the Bohr and Haldane effects studied in a model of Human-Hemoglobin. European Journal of Physiology, 408(2), S13.

[19] MacDonald, R. (1977). Red cell 2, 3-diphosphoglycerate and oxygen affinity. Anaesthesia, 32(6), 544-553.

[20] Goodnough, L. T., et al. (1999). Transfusion medicine: Blood transfusion - first of two parts. New England Journal of Medicine, 340(6), 438-447.

[21] Carson, J. L., et al. (2012). Red blood cell transfusion: a clinical practice guideline from the AABB. Annals of internal medicine, 157(1), 49-58.

[22] Goodnough, L. T., Monk, T. G., \& Brecher, M. E. (1996). Autologous Blood Procurement in the Surgical Setting: Lessons Learned in the Last 10 years. Vox Sanguinis, 71(3), 133-141.

[23] Goodnough, L. T., et al. (1999). Transfusion medicine: Blood conservation second of two parts. New England Journal of Medicine, 340(7), 525-533.

[24] Spahn, D. R., \& Goodnough, L.T. (2013). Alternatives to blood transfusion. The Lancet, 381(9880), 1855-1865.

[25] Canadian Standards Association (CSA). (2015). CAN/CSA-Z902-15 Blood and Blood Components. Retrieved from http://shop.csa.ca/en/canada/blood-and-bloodcomponents/cancsa-z902-15/invt/27020812015

[26] Canadian Society for Transfusion Medicine (CSTM). Provincial Resources. Available at http://www.transfusion.ca/Resources/Shared-Resources/Provincial

[27] Canadian Blood Services (CBS). (2015). Circular of information for the use of human blood components. Retrieved from https://blood.ca/sites/default/files/RedBloodCellsLeukocytesReduced.pdf

[28] Hurtado C., et al. (2000). Quality analysis of blood components obtained by automated buffy-coat layer removal with a top \& bottom system. Haematologica, 85(1), 390-395.

[29] Levin E., et al. (2008). Implementation of buffy coat platelet component production: Comparison to platelet-rich plasma platelet production. Transfusion, 48(1), 2331-2337.

[30] Semple E., et al. (2012). Transfusion reactions: A comparative observational study of blood components produced before and after implementation of semiautomated production from whole blood. Transfusion, 52(1), 2683-2691.

[31] Acker, J. P., et al. (2014). A quality monitoring program for red blood cell components: In vitro quality indicators before and after implementation of semiautomated processing. Transfusion, 54(10), 2534-2543.

[32] Hess, J. R., et al. (2009). Blood components: Red blood cell hemolysis during blood bank storage: Using national quality management data to answer basic scientific questions. Transfusion, 49(12), 2599-2603.

[33] Rous, P., \& Turner, J. R. (1916). The preservation of living red blood cells in vitro I: Methods of preservation. The Journal of experimental medicine, 23(2), 219-237. [34] Acker, J. P., Croteau, I. M. \& Yi, Q. L. (2012). An analysis of the bias in red blood cell hemolysis measurement using several analytical approaches. Clinica Chimica Acta, 413(21), 1746-1752.

[35] Ashby, W. (1919). The determination of the length of life of transfused blood corpuscles in man. The Journal of experimental medicine, 29(3), 267-281. 
[36] Ebaugh Jr, F. G., et al. (1953). The use of radioactive chromium 51 as an erythrocyte tagging agent for the determination of red cell survival in vivo. Journal of Clinical Investigation, 32(12), 1260-1276.

[37] Ross, J. F., \& Milan A. C. Effect of storage of citrated blood on the survival of transfused erythrocytes." Journal of the American Medical Association, 123(13), 827829.

[38] Ross, J. F., et al. (1947). The in vitro preservation and post-transfusion survival of stored blood. Journal of Clinical Investigation, 26(4), 687-703.

[39] Blood products advisory committee. (2008). Issue Summary - Topic I: BEST (Biomedical Excellence for Safer Transfusion) committee report on red blood cell recovery standards. $91^{\text {st }}$ Meeting. Retrieved from http://www.fda.gov/ohrms/dockets/ac/08/briefing/2008-4355B1_1).htm

[40] Dumont, L. J., \& AuBuchon, J. P. (2008). Evaluation of proposed FDA criteria for the evaluation of radiolabeled red cell recovery trials. Transfusion, 48(6), 1053-1060.

[41] Gibson, J. G., et al. (1957). A citrate-phosphate-dextrose solution for the preservation of human blood. American journal of clinical pathology, 28(6), 569-578.

[42] Gibson, J. G., Kevy, S., \& Pennell, R. (1967). Citrate-phosphate-dextrose: An improved anticoagulant preservative solution for human blood. International Society of Blood Transfusion. 758-763.

[43] Prowse, C. V., et al. (2014). Commercially available blood storage containers. Vox sanguinis, 106(1), 1-13.

[44] Walter, C. W. (1984). Invention and development of the blood bag. Vox sanguinis, 47(4), 318-324.

[45] Moore, G. L., \& Batsakis, J. G. (1987). Additive solutions for better blood preservation. CRC Critical reviews in clinical laboratory sciences, 25(3), 211-229.

[46] Högman, C. F., et al. (1983). Red cell suspensions in SAGM medium further Effects. Vox sanguinis, 45(3), 217-223.

[47] Amin, M., et al. (2004). The societal unit cost of allogenic red blood cells and red blood cell transfusion in Canada. Transfusion, 44(10), 1479-1486.

[48] Högman, C. F., et al. (1978). Red blood cell preservation in protein-poor media: I. Leukocyte enzymes as a cause of hemolysis. Transfusion, 18(2), 233-241.

[49] Heaton, W. A. L., et al. (1994). Effects of 3-5 $\log _{10}$ pre-storage leucocyte depletion on red cell storage and metabolism. British journal of haematology, 87(2), 363368.

[50] Rapoport, S., \& Wing, M. (1947). Dimensional, osmotic, and chemical changes of erythrocytes in stored blood. I. Blood preserved in sodium citrate, neutral, and acid citrate-glucose (ACD) mixtures. Journal of Clinical Investigation, 26(4), 591-615. [51] Gabrio, B. W., Stevens Jr., A. R., \& Finch, C. A. (1954). Erythrocyte preservation. II. A study of extra-erythrocyte factors in the storage of blood in acidcitrate-dextrose. Journal of Clinical Investigation, 33(2), 247-251.

[52] Gabrio, B. W., et al. (1954). Erythrocyte preservation. I. The relation of the storage lesion to in vivo erythrocyte senescence. Journal of Clinical Investigation. 33(2), 242.

[53] Gibson, J. G., et al. (1956). The influence of extracellular factors involved in the collection of blood in ACD on maintenance of red cell viability during refrigerated storage. American journal of clinical pathology, 26(8), 855-873. 
[54] Lion, N., et al. (2010). Stored red blood cells: a changing universe waiting for its map(s). Journal of proteomics, 73(3), 374-385.

[55] Hess, J. R. (2010). Red cell storage. Journal of proteomics, 73(3), 368-373.

[56] Bennett-Guerrero, E., et al. (2007). Evolution of adverse changes in stored

RBCs. Proceedings of the National Academy of Sciences, 104(43), 17063-17068.

[57] Ruddell J. P., et al. (1998). Effect of 24 hours of storage at $25^{\circ} \mathrm{C}$ on the in vitro storage characteristics of CPDA-1 packed red blood cells. Transfusion, 38(1), 424-428. [58] Dumaswala, U. J., et al. (1999). Protein and lipid oxidation of banked human erythrocytes: Role of glutathione. Free Radical Biology and Medicine, 27(1), 10411049.

[59] Hess, J. R. (2010). Red cell changes during storage. Transfusion and Apheresis Science, 43(1), 51-59.

[60] Du Preez, F. B., et al. (2008). A comparative analysis of kinetic models of erythrocyte glycolysis. Journal of theoretical biology, 252(3), 488-496.

[61] Hess, J. R., et al. (2005). Interlaboratory comparison of red-cell ATP, 2, 3diphosphoglycerate and haemolysis measurements. Vox sanguinis, 89(1), 44-48.

[62] Beutler, E., \& Wood, L. (1969). The in vivo regeneration of red cell 2, 3diphosphoglyceric acid (DPG) after transfusion of stored blood. The Journal of laboratory and clinical medicine, 74(2), 300-304.

[63] D'Alessandro, A., et al. (2010). Red blood cell storage: the story so far. Blood Transfusion, 8(2), 82-88.

[64] Wolfe, L. C. (1989). Oxidative injuries to the red cell membrane during conventional blood preservation. Seminars in hematology, 26(4), 307-312.

[65] Racek, J., et al. (1997). Influence of antioxidants on the quality of stored blood. Vox sanguinis, 72(1), 16-19.

[66] Chaudhary, R., \& Katharia, R. (2011). Oxidative injury as contributory factor for red cells storage lesion during twenty-eight days of storage. Blood Transfusion, 10(1), 59-62.

[67] Dumaswala, U. J., et al. (2000). Glutathione loading prevents free radical injury in red blood cells after storage. Free radical research, 33(5), 517-529.

[68] D’Amici, G. M., Rinalducci, S. \& Zolla, L. (2007). Proteomic analysis of RBC membrane protein degradation during blood storage. Journal of Proteome Research, 6(1), $3242-3255$.

[69] Kriebardis, A. G., et al. (2007). Progressive oxidation of cytoskeletal proteins and accumulation of denatured hemoglobin in stored red cells. Journal of Cellular and Molecular Medicine, 11(1), 148-55.

[70] Karon B. S., et al. (2009). Changes in band 3 oligomeric state precede cell membrane phospholipid loss during blood bank storage of red blood cells. Transfusion, 49(1), 1435-42.

[71] Low P. S., Rathinavelu P., \& Harrison M.L. (1993). Regulation of glycolysis via reversible enzyme binding to the membrane protein, band 3. Journal of Biological Chemistry, 268(1), 14627-14631.

[72] Antonelou, M. H., et al. (2012). Effects of pre-storage leukoreduction on stored red blood cells signaling: a time-course evaluation from shape to proteome. Journal of proteomics, 76(1), 220-238. 
[73] Antonelou, M. H., Kriebardis A. G., \& Papassideri, I. S. (2010). Aging and death signalling in mature red cells: From basic science to transfusion practice. Blood Transfusion, 8(3), 39-47.

[74] Greenwalt, T. J. (2006). The how and why of exocytic vesicles. Transfusion, 46(1), 143-52.

[75] Salzer U., et al. (2008). Vesicles generated during storage of red cells are rich in the lipid raft marker stomatin. Transfusion, 48, 451-62.

[76] Frank, S. M., et al. (2013). Decreased erythrocyte deformability after transfusion and the effects of erythrocyte storage duration. Anesthesia and Analgesia, 116, 975-981. [77] Högman C. F., et al. (1985). Studies on the mechanism of human red cell loss of viability during storage at +4 degrees $C$ in vitro. I. Cell shape and total adenylate concentration as determinant factors for posttransfusion survival. Vox Sanguinis, 48, 257-68.

[78] Aubron, C., et al. (2013). Age of red blood cells and transfusion in critically ill patients. Annals of intensive care, 3(2).

[79] Rapoport, S., \& Luebering, J. (1950). The formation of 2, 3-diphosphoglycerate in rabbit erythrocytes: The existence of a diphosphoglycerate mutase. Journal of Biological Chemistry, 183(2), 507-516.

[80] Holme, S. (2005). Current issues related to the quality of stored RBCs. Transfusion and Apheresis Science, 33(1), 55-61.

[81] Benesch, R., \& Benesch, R. E. (1969). Intracellular organic phosphates as regulators of oxygen release by haemoglobin. Nature, 221, 618-622.

[82] Bessis, M., Weed, R. I., \& Leblond, P. F. (Eds). (2012). Red cell shape: physiology, pathology, ultrastructure. Springer Science \& Business Media.

[83] Furchgott, R. F. (1940). Disk-sphere transformation in mammalian red cells. Journal of Experimental Biology, 17(1), 30-44.

[84] Gárdos, G., Szasz, I., \& Arky, I. (1966). Structure and function of erythrocytes. I. Relation between the energy metabolism and the maintenance of biconcave shape of human erythrocytes. Acta Biochim Biophys Acad Sci Hung, 1, 253-266.

[85] Usry, R. T., Moore, G. L., \& Manalo, F. W. (1975). Morphology of stored, rejuvenated human erythrocytes. Vox Sanguinis, 28(3), 176-183.

[86] Jones, K. W. (2009). Evaluation of cell morphology and introduction to platelet and white blood cell morphology. Clinical Hematology and Fundamentals of Hemostasis, 93-116.

[87] Palmer, L., et al. (2015). ICSH recommendations for the standardization of nomenclature and grading of peripheral blood cell morphological features. International journal of laboratory hematology, 37(3), 287-303.

[88] Longster, G. H., et al. (1972). Scanning electron microscope studies of red cell morphology. Vox Sanguinis, 22(2), 161-170.

[89] Tchir, J. D. R., Acker, J. P., \& Holovati, J. L. (2013). Rejuvenation of ATP during storage does not reverse effects of the hypothermic storage lesion. Transfusion, 53(12), 3184-3191.

[90] Berezina, T. L., et al. (2002). Influence of storage on red blood cell rheological properties. Journal of surgical research. 102(1), 6-12.

[91] Wolkers, W. F., et al. (2002). In situ assessment of erythrocyte membrane properties during cold storage. Molecular Membrane Biology, 19, 59-65. 
[92] La Celle, P. L. (1969). Alteration of deformability of the erythrocyte membrane in stored blood. Transfusion, 9(5), 238-245.

[93] Almizraq, R., et al. (2013). Storage of red blood cells affects membrane composition, microvesiculation, and in vitro quality. Transfusion, 53(10), 2258-2267.

[94] Karkouti, K. (2014). From the Journal archives: The red blood cell storage lesion: past, present, and future. Canadian Journal of Anesthesia, 61(6), 583-586.

[95] Marik, P. E., \& Sibbald, W. J. (1993). Effect of stored-blood transfusion on oxygen delivery in patients with sepsis. Jama, 269(23), 3024-3029.

[96] Purdy, F. R., Tweeddale, M. G, \& Merrick, P. M. (1997). Association of mortality with age of blood transfused in septic ICU patients. Canadian Journal of Anaesthesia, 44(12), 1256-1261.

[97] Lelubre, C., \& Vincent, J. -L. (2013). Relationship between red cell storage duration and outcomes in adults receiving red cell transfusions: a systematic review. Critical care, 17(2), R66.

[98] Wang, D., et al. (2012). Transfusion of older stored blood and risk of death: a meta-analysis. Transfusion, 52(6), 1184-1195.

[99] Remy, K. E., et al. (2016). Transfusion of recently donated (fresh) red blood cells (RBCs) does not improve survival in comparison with current practice, while safety of the oldest stored units is yet to be established: a meta-analysis. Vox Sanguinis, 111, 4354.

[100] Flegel, W. A., Natanson, C., \& Klein, H. G. (2014). Does prolonged storage of red blood cells cause harm?. British journal of haematology, 165(1), 3-16.

[101] Hopewell, S., et al. (2013). A systematic review of the effect of red blood cell transfusion on mortality: Evidence from large-scale observational studies published between 2006 and 2010. BMJ open, 3(5), e002154.

[102] Steiner, M. E., et al. (2015). Effects of red-cell storage duration on patients undergoing cardiac surgery. New England Journal of Medicine, 372(15), 1419-1429. [103] Lacroix, J., et al. (2015). Age of transfused blood in critically ill adults. New England Journal of Medicine, 372(15), 1410-1418.

[104] Heddle, N. M., et al. (2016). Effect of Short-Term vs. Long-Term Blood Storage on Mortality after Transfusion. New England Journal of Medicine, 375(20), 1937-1945. [105] Vandromme, M. J., McGwin, G., \& Weinberg, J. A. (2009). Blood transfusion in the critically ill: Does storage age matter?. Scandinavian journal of trauma, resuscitation and emergency medicine, 17(1), 35-41.

[106] Meinke, M., et al. (2007). Optical properties of platelets and blood plasma and their influence on the optical behavior of whole blood in the visible to near infrared wavelength range. Journal of biomedical optics, 12(1), 0140241-0140249.

[107] Şahin, D., \& Ilan, B. (2013). Radiative transport theory for light propagation in luminescent media. JOSA A, 30(5), 813-820.

[108] Bosschaart, N., et al. (2014). A literature review and novel theoretical approach on the optical properties of whole blood. Lasers in medical science. 29(2), 453-479.

[109] Chatterjee, D. K., Fong, L. S., \& Zhang, Y. (2008). Nanoparticles in photodynamic therapy: an emerging paradigm. Advanced drug delivery reviews, 60(15), 1627-1637.

[110] Faber, D. J., et al. (2004). Oxygen saturation-dependent absorption and scattering of blood. Physical review letters, 93(2), 0281021-0281024. 
[111] Shrestha, T. B., et al. (2012). Stem cell-based photodynamic therapy. Photochemical \& Photobiological Sciences. 11(7), 1251-1258.

[112] Sevick, E. M., et al. (1991). Quantitation of time-and frequency-resolved optical spectra for the determination of tissue oxygenation. Analytical biochemistry, 195(2), 330351.

[113] Welch, A. J., et al. (2011). Definitions and overview of tissue optics. In A. J. Welch, (Ed.), Optical-thermal response of laser-irradiated tissue (27-64). Netherlands: Springer.

[114] Baker, W. B., et al. (2014). Modified Beer-Lambert law for blood flow. Biomedical optics express, 5(11), 4053-4075.

[115] Galik, S. (2011). Cell Biology OLM. Beer_Lambert. Retrieved from http://cellbiologyolm.stevegallik.org/node/8

[116] Zijlstra, W. G., Buursma, A., \& van Assendelft, O. W. (2000). Visible and near infrared absorption spectra of human and animal haemoglobin. Utrecht: VSP.

[117] Prahl, S. (1999). Optical absorption of hemoglobin. Oregon Medical Laser Center. Retrieved from http://omlc. ogi. edu/spectra/hemoglobin/index. html 15 [118] Chan, E. D., Chan, M. M. \& Chan, M. M. (2013). Pulse oximetry: understanding its basic principles facilitates appreciation of its limitations. Respiratory medicine, 107(6), 789-799.

[119] Shapiro, H. M. (2005). Practical flow cytometry. Chapter 2. Hoboken, NJ: John Wiley \& Sons.

[120] Dawe, G. S., et al. Light microscopy. (2006). In Dokland, T. (Ed.), Techniques in microscopy for biomedical applications (Chapter A). Singapore: World Scientific.

[121] Harmening, D. M. (2009). Clinical hematology and fundamentals of hemostasis. Philadelphia, PA: FA Davis Company.

[122] Catalogue number 22-122-911. Package insert for Hema 3 Stain set (840021(R3)). Middletown VA: Fisher Scientific.

[123] Prahl, S. (1999). Optical property measurements using the inverse addingdoubling program. Retrived from http://omlc. ogi. edu/pubs/pdf/man_iad. pdf, Accessed Jan. 9, 2017.

[124] Kanias, T., \& Acker, J. P. (2010). Mechanism of hemoglobin-induced cellular injury in desiccated red blood cells. Free Radical Biology and Medicine, 49(4), 539-547. [125] Zwart, A., et al. (1996). Recommendations for reference method for haemoglobinometry in human blood (ICSH standard 1995) and specifications for international haemiglobinocyanide standard. Journal of clinical pathology, 49(4), 271274.

[126] Delost, M. (2014). Blood Gas and Critical Care Analyte Analysis. In R. L. Chatburn, et al. (Eds.) Equipment for respiratory care. (Chapter 6). Burlington, MA: Jones \& Bartlett.

[127] Ewing G. W. (1985). Instrumental methods of chemical analysis (5th Ed). New York, NY: McGraw-Hill.

[128] TCS Scientific Corporation. (2007). The HEMOX Analyser. Retreived from http://tcssci.com/hemox/index.htm, Accessed Jan. 9, 2017.

[129] Guarnone, R., Centenara, R., \& Barosi, G. (1995). Performance characteristics of Hemox-Analyzer for assessment of the hemoglobin dissociation curve. Haematologica, 80(5), 426-430. 
[130] Watson, J. V. (1999). The early fluidic and optical physics of cytometry. Cytometry, 38(1), 2-14.

[131] Macey, M. G. (2007). Flow cytometry: Principles and applications. Chapter 1. Louisville KY: Humana.

[132] Crosland-Taylor, P. J. (1953). A device for counting small particles suspended in a fluid through a tube. 37-38.

[133] Basiji, D. (2005). Multispectral imaging in flow: A technique for advanced cellular studies. In L. A. Sklar (Ed.). Flow Cytometry for Biotechnology. 57-67. New York, NY: Oxford University Press.

[134] Brunsting, A., \& Mullaney. P. F. (1974). Differential light scattering from spherical mammalian cells. Biophysical journal, 14(6), 439-453.

[135] Biosciences, B. D. (2000). Introduction to Flow Cytometry: A learning guide.

Part 1. Retrieved from

http://www.bdbiosciences.com/immunocytometry_systems/support//training//flow_cytome try_learning guide.pdf. Accessed Jan. 9, 2017.

[136] Garratty, G., \& Arndt., P. (1995). Applications of flow cytofluorometry to transfusion science. Transfusion, 35(2), 157-178.

[137] Stewart, A., et al. (2005). The application of a new quantitative assay for the monitoring of integrin-associated protein CD47 on red blood cells during storage and comparison with the expression of CD47 and phosphatidylserine with flow cytometry. Transfusion, 45(9), 1496-1503.

[138] Lee, F. Biology II. (Chapter 7.8.3). Retreived from

http://www.ubooks.pub/Books/ON/B0/E26R6789/PC8S3U25.html. Accessed Jan. 9, 2017. OpenStax College.

[139] Amnis ${ }^{\circledR}$ Imaging flow cytometers. Retreived from http://www.emdmillipore.com/CA/en/life-science-research/cell-analysis/amnis-imagingflow-cytometers/Q6ub.qB.m3UAAAFLCKIp.ygJ,nav. Accessed Jan. 9, 2017.

[140] McGrath, K. E., Bushnell, T. P. \& Palis, J. (2008). Multispectral imaging of hematopoietic cells: where flow meets morphology. Journal of immunological methods. $336(2), 91-97$.

[141] George, T. C., et al. (2004). Distinguishing modes of cell death using the ImageStream ${ }^{\circledR}$ multispectral imaging flow cytometer. Cytometry Part A, 59(2), 237-245. [142] McGrath, K. E. (2015). Utilization of imaging flow cytometry to define intermediates of megakaryopoiesis in vivo and in vitro. Journal of immunological methods 423, 45-51.

[143] López-Riquelme, N., et al. (2013). Imaging cytometry for counting circulating tumor cells: comparative analysis of the CellSearch vs ImageStream systems. Apmis, 121(12), 1139-1143.

[144] Headland, S.E., et al. (2014). Cutting-edge analysis of extracellular microparticles using ImageStreamX imaging flow cytometry. Scientific reports, 4, $52371-523710$. [145] Beers, E. J., et al. (2014). Imaging flow cytometry for automated detection of hypoxia-induced erythrocyte shape change in sickle cell disease. American journal of hematology, 89(6), 598-603.

[146] Bell, A. G. (1880). On the production and reproduction of sound by light. American Journal of Science, 118, 305-324. 
[147] Rosencwaig, A., \& Gersho, A. (1976). Theory of the photoacoustic effect with solids. Journal of Applied Physics, 47(1), 64-69.

[148] Kruger, R. A., Liu, P., and Appledorn, R., C. (1995). Photoacoustic ultrasound (PAUS) - reconstruction tomography. Medical physics, 22(10), 1605-1609.

[149] Esenaliev, R. O., et al. (1997). Laser optoacoustic imaging for breast cancer diagnostics: limit of detection and comparison with x-ray and ultrasound imaging. SPIE BIOS Proceedings, 71-82.

[150] Niederhauser, J. J., et al. (2005). Combined ultrasound and optoacoustic system for real-time high-contrast vascular imaging in vivo. IEEE transactions on medical imaging, 24(4), 436-440.

[151] Zhang, E. Z., et al. (2009). In vivo high-resolution 3D photoacoustic imaging of superficial vascular anatomy. Physics in medicine and biology, 54(4), 1035-1046.

[152] Wang, X., et al. (2003). Noninvasive laser-induced photoacoustic tomography for structural and functional in vivo imaging of the brain. Nature biotechnology, 21(7), 803806.

[153] Zhang, H. F., et al. (2006). Functional photoacoustic microscopy for highresolution and noninvasive in vivo imaging. Nature biotechnology, 24(7), 848-851. [154] Li, C., \& Wang, L. H. (2009). Photoacoustic tomography and sensing in biomedicine. Physics in medicine and biology, 54(19), R59.

[155] Chen, Y., et al. (1999), The photon counting histogram in fluorescence fluctuation spectroscopy. Biophysical journal, 77(1), 553-567.

[156] Sharma, A., \& Schulman, S. G. (1999). Introduction to fluorescence spectroscopy. Hoboken, NJ: Wiley.

[157] Xu, M., \& Wang L. H. (2006). Photoacoustic imaging in biomedicine. Review of scientific instruments, 77(4), 0411011 - 04110122.

[158] Diebold, G. J., Sun, T., \& Khan, M. I. (1991). Photoacoustic monopole radiation in one, two, and three dimensions. Physical review letters, 67(24), 3384-3389.

[159] Beard, P. (2011). Biomedical photoacoustic imaging. Interface focus, 1(4), 602631.

[160] Rui, M., et al. (2010) Photoacoustic microscopy and spectroscopy of individual red blood cells. Biomedical Optics.

[161] Strohm, E. M., Berndl, E. S. L. \& Kolios, M. C. (2013). Probing red blood cell morphology using high-frequency photoacoustics. Biophysical journal, 105(1), 59-67.

[162] Hysi, E., Saha, R. K., \& Kolios, M. C. (2012). On the potential of using photoacoustic spectroscopy to monitor red blood cell aggregation. SPIE BIOS Proceedings, 8222OQ1-8222OQ10.

[163] Hu, S., \& Wang. L. H. (2013). Optical-resolution photoacoustic microscopy: auscultation of biological systems at the cellular level. Biophysical journal, 105(4), 841847.

[164] Patterson, M. P, et al. (2014). Optoacoustic characterization of prostate cancer in an in vivo transgenic murine model. J. Biomed. Opt., 19(5), 056008-056008.

[165] Juratli, M. A., et al. (2014). Real-time monitoring of circulating tumor cell release during tumor manipulation using in vivo photoacoustic and fluorescent flow cytometry. Head \& neck, 36(8), 1207-1215.

[166] Yao, J., et al. (2010). In vivo photoacoustic imaging of transverse blood flow by using Doppler broadening of bandwidth. Optics letters, 35(9), 1419-1421. 
[167] Bok, T. -H., Hysi, E., \& Kolios, M. C. (2015). High-frequency photoacoustic imaging of erythrocyte aggregation and oxygen saturation: probing hemodynamic relations under pulsatile blood flow. SPIE BIOS Proceeding, 93231Q.

[168] Zhang, H. F., et al. (2007). Imaging of hemoglobin oxygen saturation variations in single vessels in vivo using photoacoustic microscopy. Applied physics letters, 90(5), 053901-0539013.

[169] Hysi, E., Saha, R. K. \& Kolios, M. C. (2013). Photoacoustic assessment of oxygen saturation: effect of red blood cell aggregation. SPIE BIOS Proceedings.

[170] H7379 Human Hemoglobin, Lyophilized Powder. Sigma-Aldrich, St. Louis, MO, USA. Available at

http://www.sigmaaldrich.com/catalog/product/sigma/h7379? lang $=$ en\&region $=C A \& c m$ $s p=$ Insite__prodRecCold_xviews-_prodRecCold10-7. Accessed Jan. 9, 2017.

[171] Jordan, A. (2012). Blood gas: A brief anecdotal history by one who has been there. Retreived from http://www.mlo-online.com/blood-gas-a-brief-anecdotal-historyby-one-who-has-been-there.php. Accessed Jan. 9, 2017.

[172] Paxton A. (2007). Blood gas analyzers - the old and the new. CAP Today. http://www.captodayonline.com/Archives/feature_stories/080BloodTest.html

[173] Lima-Oliveira, G., et al. (2012). Impact of the phlebotomy training based on CLSI/NCCLS H03-A6-procedures for the collection of diagnostic blood specimens by venipuncture. Biochemia medica, 22(3), 342-351.

[174] Radiometer. (2017). ABL800 FLEX Blood Gas Analyser. Copenhagen, Denmark. Retrieved from http://www.radiometer.com/en/products/blood-gas-testing/abl800-flexblood-gas-analyzer. Accessed Jan. 9, 2017.

[175] Radiometer. (2017). safePICO Syringe. Copenhagen, Denmark. Retreived from http://www.radiometer.com/en/products/samplers/safepico-syringe. Accessed Jan. 9, 2017.

[176] Radiometer. (2012). ABL800 FLEX Reference Manual. Copenhagen, Denmark. Retreived from http://www.radiometer.com/en/products/blood-gas-testing/abl800-flexblood-gas-analyzer. Accessed Jan. 9, 2017.

[177] Martens, H. A. (1985). Multivariate calibration: quantitative interpretation of non-selective chemical data. Trodheim: Norwegian Institute of Technology.

[178] Amnis Corporation. (2012). Imagestream ${ }^{X}$ Mk ii Brochure. Seattle, WA, U.S.A. Retreived from https://www.amnis.com/documents/brochures/ISX-MKII\%20Brochure

Final_Web.pdf. Accessed Jan. 92017.

[179] Amnis Corporation. (2016). Imagestream ${ }^{X}$ Mk ii Training Presentation. Seattle, WA, U.S.A. Retreived from https://www.amnis.com/customer-training.html. Accessed Jan. 92017.

[180] Zuba-Surma, E. K., et al. (2007). The ImageStream System: a key step to a new era in imaging. Folia Histochem Cytobiol, 45(4), 279-290.

[181] Amnis Corporation. (2012). Imagestream ${ }^{X}$ Mk ii Application Software INSPIRE. Seattle, WA, U.S.A. Retreived from http://www.emdmillipore.com/CA/en/life-scienceresearch/cell-analysis/amnis-imaging-flow-cytometers/analysis-acquisitionsoftware/inspire-software/TMib.qB.0UIAAAFLTV8Jx34Q,nav. Accessed from Jan. 9, 2017.

[182] Amnis Corporation. (2009). Imagestream ${ }^{X}$ Mk ii Analysis Sofware IDEAS. Seattle, WA, U.S.A. Retreived from http://www.emdmillipore.com/CA/en/life-science- 
research/cell-analysis/amnis-imaging-flow-cytometers/analysis-acquisitionsoftware/ideas-software/qe2b.qB.Oq8AAAFLQM8Jx34R,nav. Accessed from Jan. 9, 2017.

[183] VisualSonics. Vevo LAZR PA Imaging System. Toronto, ON., Canada. Retreived from http://www.visualsonics.com/products/vevo-lazr-photoacoustic-imaging. Accessed Jan. 9, 2017.

[184] Lakshman, M., \& Needles, A. (2015). Screening and quantification of the tumor microenvironment with micro-ultrasound and photoacoustic imaging. Nature Methods, 12(4).

[185] VisualSonics. (2014). Vevo LAZR Manual. Toronto, ON., Canada. Retreived from http://www.ibb.cnr.it/img/Vevo_LAZR_manual.pdf. Accessed Jan. 9, 2017.

[186] VisualSonics. (2017). Vevo LAZR Brochure. Toronto, ON., Canada. Retreived from http://www.accela.eu/files/products/61/vevo-lazr-brochure-ver8-5.pdf. Acccessed Jan. 9, 2017.

[187] Hysi, E., Dopsa, D., \& Kolios, M. C. (2014). Photoacoustic tissue characterization using envelope statistics and ultrasonic spectral parameters. SPIE BIOS Proceeding.

[188] Needles, A., et al. (2013). Development and initial application of a fully integrated photoacoustic micro-ultrasound system. IEEE transactions on ultrasonics, ferroelectrics, and frequency control, 60(5), 888-897.

[189] VisualSonics. Vevo LAZR Technical Specifications. Toronto, ON., Canada.

Retreived from

http://www.visualsonics.com/sites/default/files/Vevo_LAZR_technical_specifications.pdf. Accessed Jan. 9, 2017.

[190] Forlani, L., et al. (1984). Calorimetric studies of oxyhemoglobin dissociation. I.

Reaction of sodium dithionite with oxygen. Journal of inorganic biochemistry, 20(2), 147-155.

[191] Forlani, L., et al. (1985). Calorimetric studies of oxyhemoglobin dissociation. II. Erythrocytic oxygen depletion by sodium dithionite. Journal of inorganic biochemistry, 23(2), 109-117.

[192] Bland, J. M., \& Altman, D. G. (1999). Measuring agreement in method comparison studies. Statistical Methods in Medical Research, 8(2), 135-160.

[193] Haralick, R.M., Shanmugan, K., \& Dinstein, (1973). I. Textural Features for Image Classification. IEEE Transactions on Systems, Man, and Cybernetics, Vol. SMC3, 610-621.

[194] Jordan, A., et al. (2016). Assessing the influence of component processing and donor characteristics on quality of red cell concentrates using quality control data. Vox Sanguinis, 111(1), 8-15.

[195] Saiko, Guennadi, et al. (2014). Optical detection of a capillary grid spatial pattern in epithelium by spatially resolved diffuse reflectance probe: Monte Carlo verification.

IEEE Journal of Selected Topics in Quantum Electronics, 20(2), 1-9.

[196] Retrieved from

http://img.bhs4.com/f2/b/f2b6886983f009f4355030fb5331eb709e92bf3e_large.jpg.

Accessed Jan. 92017. 